


\section{Propositions}

1. Zwitterionic polymer brushes have more plusses than minuses.

(this thesis)

2. Surface plasmon resonance measurements on zwitterionic polymer brushes are only meaningful when using dialyzed biological media.

(this thesis)

3. In contrast to what is often claimed in literature, there is no such thing as a non-fouling surface.

4. In vivo tumor targeting of nanoparticles does not exist, retention does.

5. Low-hanging fruits in science often turn out to be unripe.

6. Multi-disciplinary research can only be successful if scientists are not only able to 'walk the walk' but also to 'talk the talk'.

7. Designating soy as a healthy product leads to adverse effects on human health.

8. Growing up with animals improves emotional and physical health in adult life.

Propositions belonging to the thesis entitled:

“Romantic Surfaces - Zwitterionic Polymer Brushes for Biomedical Applications”

Esther van Andel

Wageningen, November 9, 2018 


\section{Romantic Surfaces}

\section{Zwitterionic Polymer Brushes for Biomedical Applications}

Esther van Andel 


\section{Thesis committee}

\section{Promotors}

Prof. Dr H. Zuilhof

Professor of Organic Chemistry

Wageningen University \& Research

Prof. Dr H.F.J. Savelkoul

Professor of Cell Biology and Immunology

Wageningen University \& Research

\section{Co-promotors}

Dr M.M.J. Smulders

Assistant professor, Laboratory of Organic Chemistry

Wageningen University \& Research

Dr E.J. Tijhaar

Assistant professor, Cell Biology and Immunology Group

Wageningen University \& Research

\section{Other members}

Prof. Dr J. van der Oost, Wageningen University \& Research

Prof. Dr P. Jonkheijm, University of Twente

Dr A. van Amerongen, Wageningen University \& Research

Dr P.A. Levkin, Karlsruhe Institute of Technology, Germany

This research was conducted under the auspices of the Graduate School VLAG (Advanced studies in Food Technology, Agrobiotechnology, Nutrition and Health Sciences). 


\title{
Romantic Surfaces
}

\section{Zwitterionic Polymer Brushes for Biomedical Applications}

\author{
Esther van Andel
}

\section{Thesis}

submitted in fulfilment of the requirements of the degree of doctor

at Wageningen University

by the authority of the Rector Magnificus,

Prof. Dr A.P.J. Mol,

in the presence of the

Thesis Committee appointed by the Academic Board

to be defended in public

on Friday 9 November 2018

at 4 p.m. in the Aula. 
Esther van Andel

Romantic Surfaces - Zwitterionic Polymer Brushes for Biomedical Applications,

254 pages.

$\mathrm{PhD}$ thesis, Wageningen University, Wageningen, the Netherlands (2018) With references, with summary in English

ISBN 978-94-6343-515-4

DOI $\quad 10.18174 / 459827$ 
Voor opa. 
81 


\section{Contents}

$\begin{array}{lll}\text { Chapter 1. General Introduction } & 10\end{array}$

Chapter 2. Efficient and Tunable 3D-Functionalization of Fully Zwitterionic 30 Antifouling Surface Coatings

Chapter 3. Highly specific binding on antifouling zwitterionic polymer-coated 68 microbeads as measured by flow cytometry

Chapter 4. Systematic Comparison of Zwitterionic and Non-Zwitterionic 108 Antifouling Polymer Brushes on a Bead-Based Platform

Chapter 5. Specific Protein Identification by Immunoprecipitation-Mass 148 Spectrometry Using Antifouling Microbeads

Chapter 6. Targeted Phagocytosis of Functionalized Stealth Microbeads by 184 Macrophages

$\begin{array}{lll}\text { Chapter 7. } & \text { General Discussion } & 208\end{array}$

$\begin{array}{ll}\text { Summaries } & 235\end{array}$

About the Author 243 


\section{Chapter.}

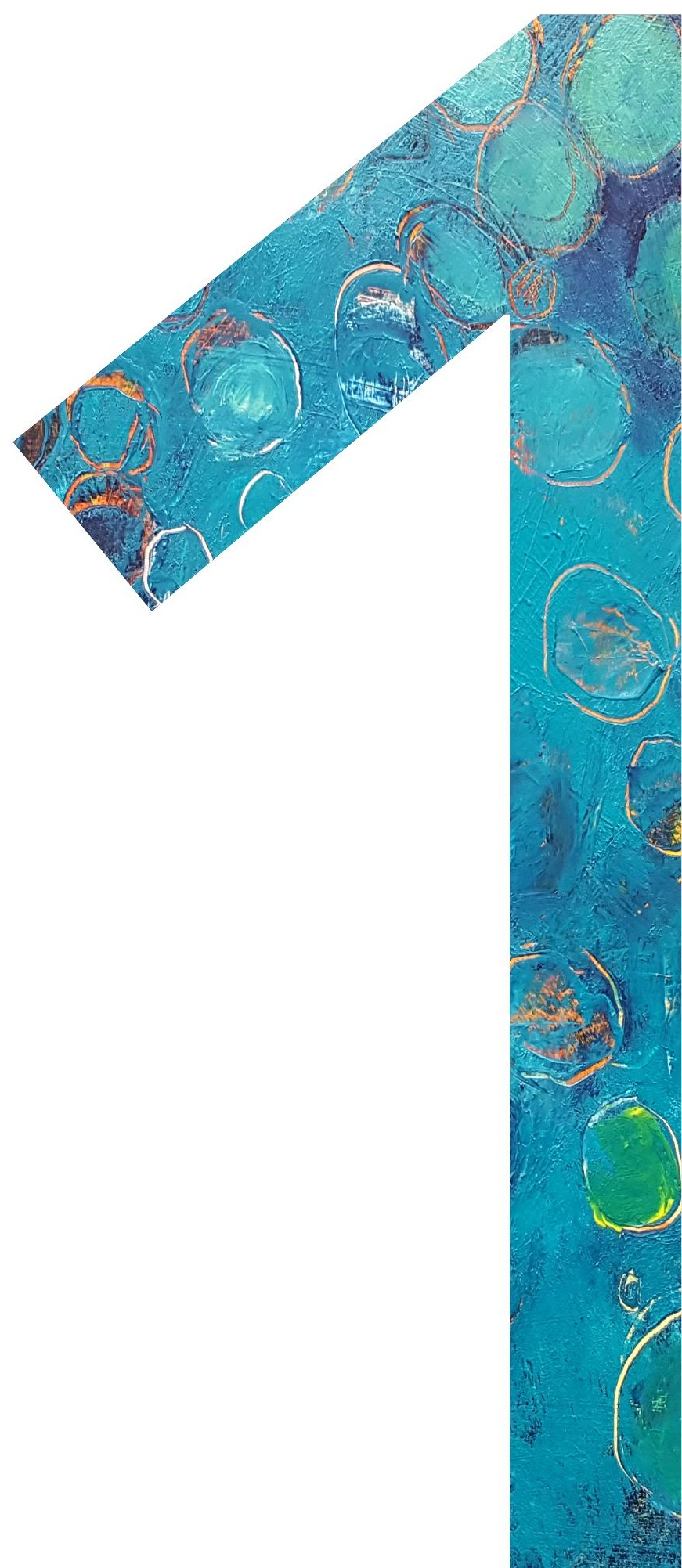




\section{General Introduction}


12 | Chapter 1 


\subsection{Fouling in biomedical applications}

Non-specific adsorption of biomolecules and microorganisms, a process often referred to as biofouling (see Figure 1.1 on page 14), is a great challenge in many biomedical applications. ${ }^{1-2}$ Complications related to implanted medical devices, for instance, often originate from fouling; protein and microbe adsorption can cause infection, thrombosis, ongoing inflammatory responses, fibrosis and even extrusion of the implant. ${ }^{3-5}$ At the implant site, proteins typically adsorb within seconds. This process is followed by a cascade of biological responses, including platelet and c-omplement activation, possibly followed by a foreign body reaction (collagenous encapsulation and thereby isolation of the implant from the body)., 6-7 Next to that, in biosensing applications (both in the clinic as well as in research), non-specific protein adsorption reduces the sensitivity of the measuring device as it leads to background signals that diminish the signal-to-noise ratio. ${ }^{8-9}$ In fact, the risk of 'false positives' and 'false negative' outcomes as a result of fouling is the major technical issue that prevents many promising biosensing systems from entering the clinic. ${ }^{10}$ The performance of drug delivery carriers is also highly affected by fouling: the adsorption of proteins on the drug carriers triggers phagocytic uptake by macrophages, causing the rapid clearance from the body, and thus hampering the drug to reach its target site (e.g. a solid tumor).$^{11-13}$ It is evident from the aforementioned examples that fouling has a tremendous impact on today's healthcare system. As a result, great efforts have been made to prevent it. ${ }^{1,5,10,14-16}$ As every fouling process begins with the spontaneous adsorption of single proteins to a surface (e.g. a sensor surface or the surface of an implant), ${ }^{6}$ the focus of research has been on preventing protein adsorption by incorporation of antifouling coatings on various surfaces. The basic principles on the quest to non-fouling coatings are outlined in this chapter.

\subsection{Antifouling coatings}

A wide range of different materials has been developed to diminish the non-specific binding of proteins. ${ }^{17-18}$ This includes self-assembled monolayers (SAMs) that are formed by the spontaneous adsorption of organic molecules onto surfaces, consisting of an anchoring group, a tail and functional group. ${ }^{19}$ But also coatings based on oligo-/poly(ethylene glycol) (OEG/ PEG), ${ }^{20-22}$ amino acids, ${ }^{23}$ polysaccharides, ${ }^{24}$ short hydroxyalkyls, ${ }^{25}$ and zwitterionic polymerbased coatings. ${ }^{1-2}$ The coatings can be prepared on various types of substrates, such as gold, ${ }^{26-27}$ glass, ${ }^{28}$ and semiconductor materials. ${ }^{29-30}$ Whitesides and co-workers reported one of the first fundamental studies on protein-repellent layers based on different SAMS on gold, ${ }^{19,}{ }^{31-32}$ leading to a set of empirical guidelines now known as the 'Whitesides rules'. ${ }^{6}$ The guidelines state that good antifouling coatings have: 1) polar functional groups, 2) hydrogen bond acceptors, 3) no hydrogen bond donors, and 4) no net charge. Although several coatings with excellent antifouling properties have been reported that do not obey these rules, ${ }^{25,33-34}$ the guiding principles have led to the development of a wide variety of antifouling materials of which PEGbased layers are most noteworthy. ${ }^{35-36}$ 


\section{Poly(ethylene glycol)}

PEG (see Scheme 1.1) has been the most widely used antifouling polymer, such that it has often been termed as the 'gold standard' for protein-resistant surfaces.6. ${ }^{6} 6$ PEGylation of nanoscopic materials like nanoparticles, drug conjugates and micelles for applications such as drug delivery, has especially drawn much attention. ${ }^{36-37}$ Despite its frequent use, PEG has several notable limitations. PEG-based coatings perform well in single-protein solutions, but their antifouling performance is limited in real-life biological fluids such as blood plasma or serum. ${ }^{27}$ Besides that, PEG-based materials are prone to oxidative damage in biological media, limiting their use in applications requiring long-term stability. ${ }^{19,}{ }^{38}$ Enzymatic degradation of low molecular-weight PEG polymers has been shown to produce toxic metabolites ${ }^{39}$ and in some cases hypersensitivity reactions, even anaphylactic shocks were observed upon in vivo administration of PEG materials. ${ }^{40}$ PEG was initially considered to be non-immunogenic, but in several cases anti-PEG antibodies have been shown to be generated as a reaction to PEGylated delivery particles. ${ }^{40-41}$ These antibodies may result in faster clearance of the PEGylated drugs in vivo, reducing the efficiency of the drug. Evidently, for both diagnostic as well as for in vivo applications there is a demand for better performing antifouling materials.

Fouling

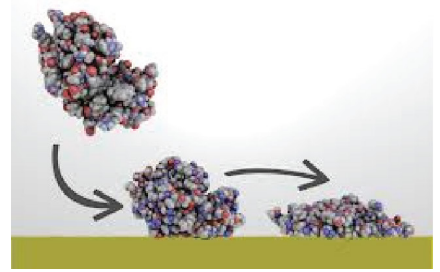

\section{Antifouling}

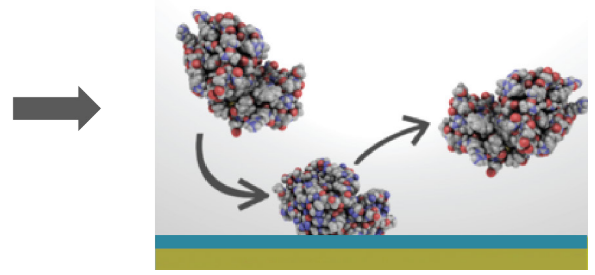

Figure 1.1 Schematic representation of the non-specific adsorption of proteins (fouling) and the repellence of proteins by means of a surface coating (antifouling). *Image adapted from http://andrewwhite.com/research/rational.

\subsubsection{Zwitterionic antifouling coatings}

\section{HYDRATION}

Zwitterionic materials have been brought forward as excellent alternative to PEG because of their biocompatibility, ${ }^{42}$ long-term stability in aqueous solutions ${ }^{30}$ and their great antifouling properties even in complex biological samples as blood serum and plasma. ${ }^{26-27}$ Zwitterionic polymers are overall electrically neutral, but their monomers bear both positive and negative charges. The positive charge is most often a quaternary ammonium ion and the negative anion can be either a sulfonate, carboxylate or phosphonate, ${ }^{6}$ see Scheme 1.1. The charged 
moieties ensure a tightly bound hydration layer and thereby a swollen polymer brush. It is widely accepted that the degree of hydration is directly correlated to the efficiency of a material to resist protein adsorption: to bind strongly to a surface, proteins have to come close, which requires the removal of water and collapse of the brush. ${ }^{17,43}$ The strongly bound water molecules prevent this from happening, and thus act as a physical and energy barrier that prevents proteins and microorganisms from adsorbing on the surface. ${ }^{44}$ Electrostatically induced solvation in zwitterionic coatings generally leads to better hydration than hydrogenbond driven hydration as observed for PEG, ${ }^{17,44}$ explaining why zwitterions outperform PEGbased materials.

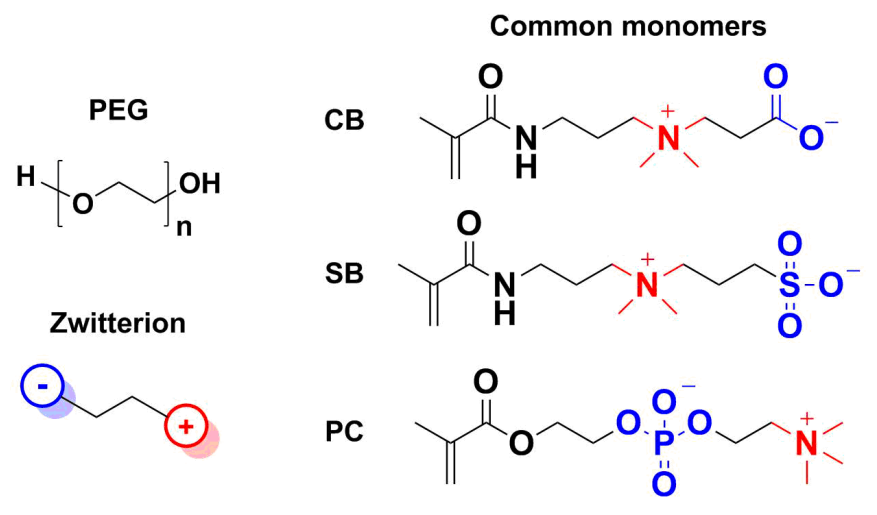

Scheme 1.1 Chemical structure of PEG and commonly used zwitterionic polymer brushes.

\section{PHOSPHOCHOLINE}

The first generation of zwitterionic antifouling materials was inspired by phosphatidylcholine lipids that are found in cell membranes. ${ }^{45}$ Phosphatidylcholine is crucial for proper functioning and communication of a cell as it ensures an inert surface at which biological interactions between (glyco)proteins can occur. ${ }^{5}$ The first phosphoryl choline (PC, or phosphocholine) inspired material was already studied back in $1978,{ }^{46}$ but the rather complex synthesis of PCbased monomers (such as 2-methacryloyloxyethyl phosphoryl choline, also often abbreviated as MPC, see Scheme 1.1) significantly hampered further development. ${ }^{5,47}$ The establishment of a refined synthesis and purification protocol for MPC by Ishihara et al., ${ }^{48}$ contributed considerably to the development of PC-based antifouling materials. Today, MPC polymers are commercially available worldwide and are utilized in numerous clinical applications such as for soft contact lenses, cardiovascular devices, and orthopedic devices like artificial hip joints. ${ }^{5}$

\section{CARBOXY- AND SULFOBETAINES}

Systematic investigations of zwitterionic materials are mostly based on sulfobetaines (SB) and carboxybetaines (CB), which can be attributed to the easy synthesis of the betaine monomers and subsequent polymers. ${ }^{49-51} \mathrm{SB}$ and $\mathrm{CB}$ materials bear a positively charged quaternary 
ammonium cation and a sulfonate or carboxylate anion, respectively. The most commonly used monomers are the SB-3 and CB-2 monomers, in which the opposite charges are separated by three or two methylene groups, respectively. ${ }^{1,52-56}$ The structure of SB is similar to that of taurine (2-aminoethane sulfonic acid), which is present in high concentration in the human body. ${ }^{57}$ The structure of a CB monomer is similar to that of glycine betaine, which is vital for the osmotic regulation of living organisms. ${ }^{58}$ As a result, materials derived from SB or CB are considered as biomimetic. ${ }^{1}$ Their biomimetic nature along with their excellent antifouling characteristics make them interesting for a wide range of applications, ${ }^{13,}{ }^{59}$ such as biosensing, ${ }^{60-61}$ drug delivery, ${ }^{12,62}$ filtration, ${ }^{51,63-64}$ as well as for antibacterial ${ }^{65}$ and marine coatings. ${ }^{15,66} \mathrm{~A}$ fundamental difference between SB and CB is that CB-based materials can be directly functionalized (mostly via EDC/NHS chemistry to attach antibodies, see below), and are therefore often used for biosensing applications. ${ }^{26,61,67}$ However, this functionalization comes at the expense of charge neutrality. ${ }^{68}$ Another difference is that the CB-based materials are $\mathrm{pH}$ sensitive: the carboxyl groups will be protonated at low $\mathrm{pH}$. Upon protonation, the zwitterionic character of the $\mathrm{CB}$ is lost, which will impair its antifouling properties. ${ }^{49} \mathrm{SB}$-based materials cannot be directly functionalized, but have a sufficient low $\mathrm{pK}_{\mathrm{a}}$ that they can be considered $\mathrm{pH}$ insensitive. ${ }^{2}$

\subsubsection{Factors influencing antifouling properties}

The aforementioned hydration layer is crucial for excellent protein repellence. Consequently, the degree to which a coating can be hydrated is considered the key parameter in antifouling efficiency. ${ }^{2,17,69}$ Whether a coating is well hydrated or not is largely determined by its chemical nature. ${ }^{44,69-71}$ It has been shown that even small changes, like the number of methylene groups between the positive and negative charge of a zwitterionic monomer, have an effect on the ability to repel proteins. ${ }^{49,72}$ The exact relationship between chemical structure and protein resistance will be extensively discussed in chapters 3 and 7. Besides the chemical nature, the physical properties of an antifouling coating also need to be considered. For example: polymer layers perform better than SAMs, ${ }^{17,27,73}$ and a higher polymer density is generally better than a low polymer coverage or density. ${ }^{74-76}$ Protein resistance of SAMs only relies on the hydration capacity of the functional groups in the topmost part of the monolayer, while for long-chain polymers both surface hydration as well as steric repulsion (due to chain flexibility) contribute to the protein-repellent behavior. ${ }^{17}$

There are two ways to attach coatings with long-chain polymers: either via grafting-from or graftingto polymerizations (see Figure 1.2). ${ }^{117}$ The grafting-to approach refers to pre-made polymers that are then tethered to the surface, either by physisorption or covalent attachment. For the grafting-from approach, initiator molecules are first installed on the surface, enabling polymer growth from the surface. Although physisorption of polymers is easy and convenient, coatings prepared by the grafting-from method lead to more stable and thicker coatings with higher packing densities. ${ }^{1,6}$ When individual polymer chains are tethered by one end of the polymer chain to an interface at high grafting density, they are referred to as 'polymer brushes'. ${ }^{77}$ In a typical grafting-from experiment, first an initiator is attached to a surface from which polymer 
chains are grown using a polymerization technique that is initiated by the immobilized initiator moieties. ${ }^{30,77}$ Especially 'living' polymerization techniques like atom transfer radical polymerization (ATRP) ${ }^{78}$ (discussed in detail below) have yielded many antifouling coatings with outstanding performance. ${ }^{1,30,33,73}$ The living character of the polymerization techniques enables the preparation of antifouling coatings with tunable thicknesses, which might be relevant for its functioning. For example, Yang et al. demonstrated that their sulfobetaine polymer brushes of $62 \mathrm{~nm}$ showed minimal fouling whereas both thinner and thicker brushes increased the amount of fouling. ${ }^{56}$

\section{A. Hydration}

Water molecule

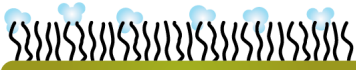

SAM

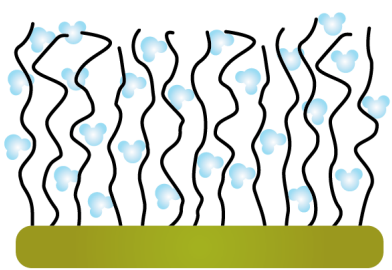

Hydrophilic polymer

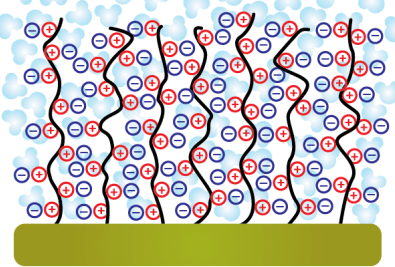

Zwitterionic polymer brush

\section{B. Grafting from vs grafting to}

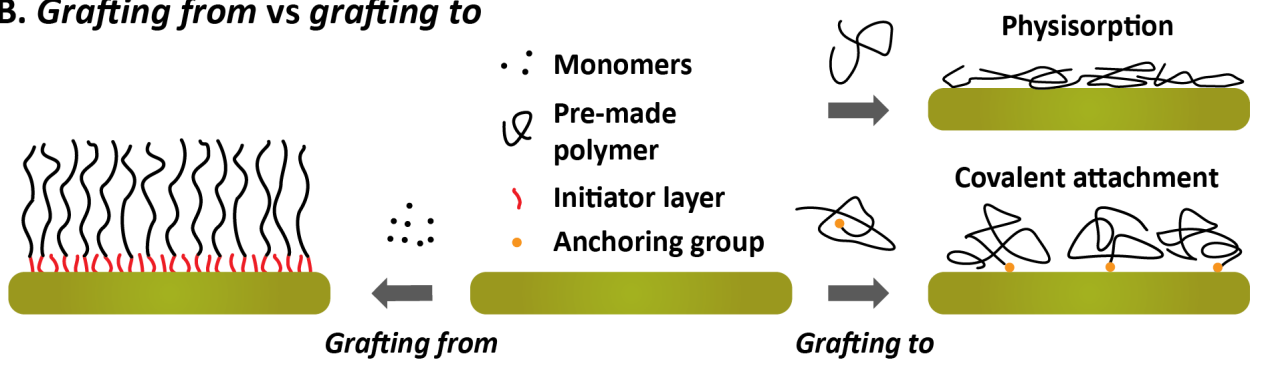

Figure 1.2 Schematic representation of (A) the relative amount of hydration using a self-assembled monolayer (SAM), a hydrophilic polymer or a zwitterionic polymer brush, and (B) the result of a grafting-from and a grafting-to polymer coating.

Environmental conditions like $\mathrm{pH},{ }^{49,79-80}$ ionic strength, ${ }^{70,81}$ type of ions, ${ }^{72}$ and temperature ${ }^{74}$ also affect the antifouling properties, as they can influence the overall charge and/or degree of hydration of the antifouling coatings. Moreover, it has been shown that the concentration, complexity, type and source of the used protein solutions (e.g. serum from different species or just from different donors) also influences the amount of protein that adsorbs on a particular coating. ${ }^{10,82}$ 


\section{Surface-initiated atom transfer radical polymerization (SI-ATRP)}

Although conventional radical polymerization is used by industry to produce annually ca. 100 million tons of different type of polymers, the architectural control in these polymers is very limited. ${ }^{83}$ Controlled (or "living") polymerization techniques, like reversible additionfragmentation chain transfer (RAFT), nitroxide-mediated polymerization (NMP) and atom transfer radical polymerization (ATRP), have been introduced since the 1980 s to fulfill the demand to prepare polymers with more complex compositions (block, gradient, alternating, etc.), topologies (stars, combs, brushes, etc.) and functionalities (see Figure 1.3) ${ }^{83-84}$ The living polymerization techniques allow for the preparation of polymers with great control over the molecular weight and over the molecular weight distribution..$^{85}$ Especially ATRP, which was developed independently by Matyjaszewski ${ }^{36-87}$ and Sawamoto's group, ${ }^{88}$ has received great attention since its discovery in 1995. The success of ATRP can be attributed to a large choice of (commercially) available initiators, the tolerance towards a wide range of monomers, and to the fact that the polymerization can be carried out in a variety of solvents and temperatures, including water at room temperature. ${ }^{85}$

\section{A. Polymer compositions}

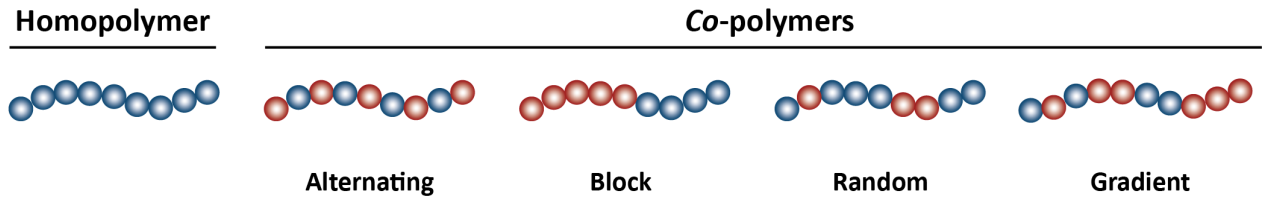

\section{B. Polymer topologies}

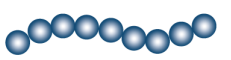

Linear

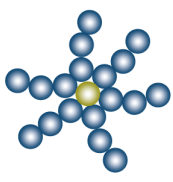

Star

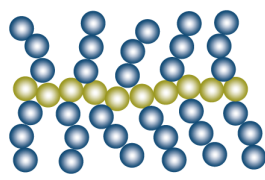

Brush/comb

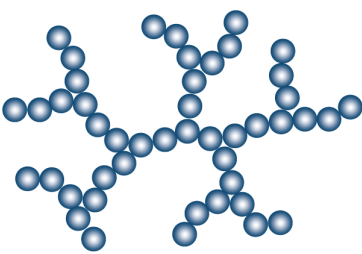

Branched

Figure 1.3 Schematic representation of (A) several polymer compositions, and (B) polymer topologies.

The ATRP mechanism is depicted in Scheme 1.2. The reaction components of a typical ATRP reaction consist of an organic halide $\left(\mathrm{P}_{\mathrm{n}}-\mathrm{X}\right)$ as the ATRP initiator/macromolecular species, and a transition metal in the low-oxidation state that is embedded in a complex with one or more ligands $\left(\mathrm{M}_{\mathrm{t}}^{\mathrm{m}} / \mathrm{L}\right)$. The transition metal/ligand complex is often referred to as the 'activator'. After abstraction of the halogen from $\mathrm{P}_{\mathrm{n}}-\mathrm{X}$, the 'deactivator' is generated as an oxidized halogenated 
transition metal complex $\left(X-M_{t}^{m+1} / L\right)$ together with the formed radical $\left(P_{n}^{*}\right)$, which can react with the provided monomer $(\mathrm{M})$ to form $\mathrm{P}_{\mathrm{n}+1} \cdot{ }^{83,85}$ The active radical is then converted into a dormant species by reacting with the deactivator to form $\mathrm{P}_{\mathrm{n}+1}-\mathrm{X}$, from which the cycle can be repeated. The 'living' nature of ATRP is established by the equilibrium between propagating radicals and dormant chains, in which the equilibrium is strongly shifted towards the dormant species (i.e. $\mathrm{k}_{\text {deact }}>>\mathrm{k}_{\text {act }}$ ) ${ }^{89}$ The shifted equilibrium favors the deactivation of propagating radicals and thereby suppresses the undesirable termination reactions $\left(\mathrm{k}_{\mathrm{t}}\right) \cdot{ }^{85}$

Scheme 1.2 ATRP mechanism.

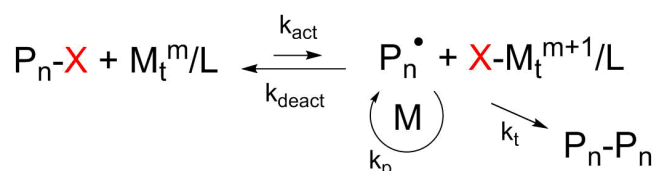

Surface-initiated atom transfer radical polymerization (SI-ATRP), in which ATRP is used to grow polymer brushes from a surface, maintains all attributes of traditional ATRP. ${ }^{90}$ The relative simplicity of the required initiators together with recent progress in surface modification techniques has made it possible to grow polymer brushes from virtually any surface, ${ }^{77,90}$ including that of biomolecules. ${ }^{91-92}$ The controlled nature of SI-ATRP allows for the preparation of dense polymer brushes that are highly uniform and have predefined thickness, architecture and functionality. ${ }^{77,90}$ As a result, SI-ATRP has been established as an excellent technique to grow polymer brushes, which can be used in the biomedical field with applications in separation, antibacterial and/or antifouling materials, controlled cell adhesion, and biosensing. ${ }^{10,15,59}$ SIATRP has been used throughout the research chapters of this thesis.

\subsection{Romantic surfaces}

For some applications, like medical implants, an antifouling coating alone would suffice. However, diagnostic applications require the presence of recognition units (e.g. antibodies, aptamers, peptides, etc.) to be able to bind a target analyte. The term 'romantic surface' was introduced to describe a surface that has both antifouling properties as well as built-in recognition units (see Figure 1.4). ${ }^{93}$ It is considered romantic because it repels everything that it is not interested in, but strongly binds to only that single entity it 'desires'. Such a romantic surface is highly valuable for diagnostic purposes, as it can significantly improve the signal-to-noise ratios of biosensing systems. ${ }^{94-95}$ The trend towards point-of-care (POC) devices especially stresses the demand for such surfaces, as measurements with POC devices typically require small volumes with unprocessed biological fluids (e.g. full blood, urine etc.). ${ }^{96}$ Sensitive measurements without background interference are therefore crucial. Romantic surfaces are also desired for research laboratory procedures, such as for the isolation and detection of cells and proteins to improve their purity and sensitivity of detection. In the last decade, there have been various examples ${ }^{60,67,97-100}$ in which zwitterionic coatings have been employed to fulfill the need of romantic surfaces. However, the immobilization of recognition units is still challenging and often occurs at the expense of the antifouling properties. ${ }^{61,68,100}$ 


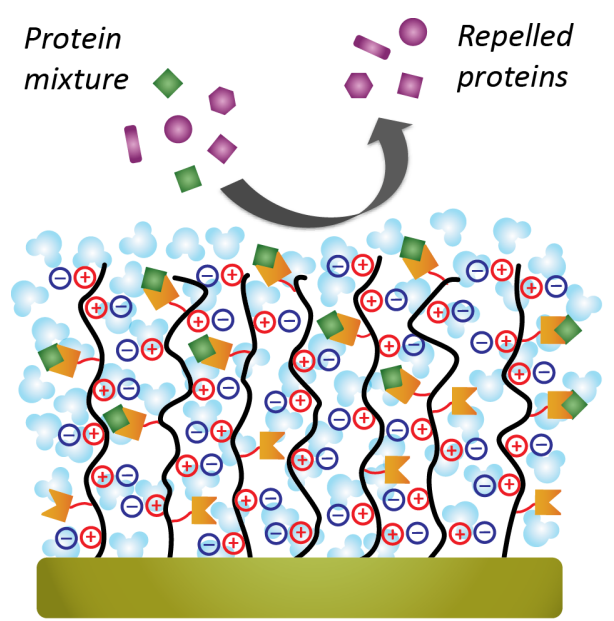

\author{
Water molecule \\ Non-target molecules \\ Analyte \\ Recognition unit \\ $\oplus \Theta \quad$ Zwitterionic monomer
}

ROMANTIC SURFACE

Figure 1.4 Schematic representation of a romantic surface.

\title{
1.3.1 Surfaces and surface attachment chemistries
}

The first step in the preparation of antifouling zwitterionic polymer brushes is the instalment of an ATRP initiator from which the brushes can be grown. The choice of surface substrate is often dictated by the specific application in which the antifouling coating needs to be embedded. Biosensors for example often require a specific type of surface substrate, such as gold (or silver) for surface plasmon resonance (SPR), ${ }^{101}$ silicon or silicon nitride for micro-ring resonators, ${ }^{102-103}$ and (among others) glass or polydimethylsiloxane (PDMS) for microfluidic chips. ${ }^{104}$ Many different types of surface chemistries ${ }^{93,105}$ have been developed, all with their own characteristic features in terms of stability, reproducibility, commercial availability of reactants and surface preference. Thiols on gold and silanes on oxide surfaces are well-known and frequently used due to their simple preparation methods. They do, however, suffer significantly from degradation under conditions typically used in biosensing measurements, such as exposure to PBS buffers. ${ }^{105}$ Surface modifications based on the attachment of alkynes or alkenes on oxygenfree silicon-based materials often lead to stable and well-ordered monolayers, ${ }^{105-106}$ but they are less easily prepared by a non-(surface) chemist. For some types of surface chemistry, there are commercially available ATRP initiators that can be readily attached in an easy one-step procedure. Examples are 11-mercaptoundec-1-yl 2-bromo-2-methylpropionate for direct modification on gold, or 11-(2-bromoisobutyrate)-undecyl-1-phosphonic acid for attachment onto oxide surfaces. ${ }^{107-108}$ For other surfaces, multi-step procedures have to be designed in which the surfaces are first functionalized with a surface-active precursor molecule, on which subsequently an ATRP initiator can be installed. ${ }^{109}$ 
Antifouling surfaces have been extensively studied on flat surfaces, the incorporation of antifouling coatings is, however, also highly desirable on nanoparticles, drug carriers, microbeads or soft materials such as hydrogels. Nano- and micron-sized particles are frequently used for biomedical applications including, imaging, drug delivery, cell sorting, biomolecule separation and quantification. ${ }^{10-111}$ The non-specific adsorption of proteins, cells or bacteria can significantly hamper the performance of the particles. Methodologies to efficiently protect these particles from fouling using zwitterionic coatings are therefore highly desirable.

\subsubsection{Functionalization of antifouling zwitterionic polymer brushes}

The most commonly used method to introduce functionality to antifouling zwitterionic polymer brushes is the direct modification of the carboxylic acid groups of $\mathrm{CB}$ monomers using EDC/NHS chemistry. 26, 52, 61, 97 This method allows for the immobilization of aminebearing (bio)molecules including peptides and proteins. It has, for example, successfully been used to incorporate anti-ALCAM antibodies to capture ALCAM, a glycoprotein present on (among others) epithelial cells. ${ }^{52,97}$ The disadvantage of this type of functionalization is that a fraction of the zwitterionic character is lost, which renders the surface more prone to fouling. ${ }^{68}$ Another approach is to make use of the terminal halogen atom that is left on the polymer chain end after ATRP by a method called top functionalization or chain end modification. ${ }^{112-114}$ This method can be used to install a large variety of functional groups, including amines and azides, in which the latter can be used for click chemistry (simple, high-yielding reactions that can be performed under mild conditions). ${ }^{115}$ This type of functionalization can be used on any ATRP-grown polymer brush, but is limited to relatively thin brushes (max 20-25 nm) since the end groups for thicker brushes tend to get buried within the brush. ${ }^{116}$ Besides, the amount of incorporated recognition elements is inherently low and cannot be controlled. ${ }^{13}$ Another strategy is to copolymerize a non-zwitterionic monomer with functional groups, such as epoxy units that can efficiently react with amine-bearing molecules. ${ }^{100,117}$ However, the more functional groups are built in, the greater the negative impact on the antifouling performance.

\subsection{Thesis outline}

As outlined above, antifouling zwitterionic polymer brushes have emerged as excellent candidates to prevent the non-specific adsorption of biomolecules within various biomedical applications. However, the criteria that these coatings need to fulfill - like long-term stability, antifouling in complex samples as full blood and zero immunogenicity - push for the development of even better antifouling coatings that can be attached onto a wide variety of substrates. The aim of this thesis is to develop functional zwitterionic polymer brush coated surfaces, and thereby approaching the 'romantic surface' principle, useful for biomedical applications.

In chapter 2, a novel approach is presented in which zwitterionic character and functionality are unified within a single monomer. The copolymerization of a standard sulfobetaine 
monomer with our new sulfobetaine with built-in azide moiety allows for the formation of 3D-functionalizable antifouling coatings with a tunable amount of functional groups. The incorporation of 1-5\% of this azido-sulfobetaine, functionalized with biotin via click chemistry, is shown to enable the highly selective binding of streptavidin without the interference of nonspecifically bound proteins.

Most of the literature regarding antifouling coatings considers flat surfaces. However, particles, both in the nanometer as well as in the micrometer scale, are often used in biomedical applications and also suffer from fouling. Chapter 3 describes the preparation of antifouling zwitterionic polymer-coated beads and how to install a variety of functional groups on them that can be used for further functionalization. Flow cytometry is used to evaluate both the specific as well as non-specific binding of proteins.

The bead-based system developed in chapter 3 offers a powerful platform to deepen the understanding of the exact relationship between monomer structure and antifouling performance. Chapter 4 describes the comparison of several types of antifouling coatings with a special focus on the influence of the carbon spacer length between the opposite charges of the zwitterionic monomers.

In chapter 5 the combination of the novel azido monomer (as described in chapter 2) and the antifouling beads (as described in chapter 3) is described in order to create antifouling immunoprecipitation beads. Such beads can be used to decrease the background signals due to non-specific protein binding in mass spectrometry-based proteomics. The chapter describes the immobilization of antibodies to the antifouling beads, which are subsequently used to pull-down target proteins together with their interaction partners that are then analyzed by mass spectrometry.

The ability to be 'invisible' for the immune system is highly desirable for applications such as drug delivery or indwelling devices. Chapter 6 describes how a zwitterionic coating on beads can prevent phagocytosis by macrophages and that the phagocytosis of beads can be triggered by functionalization of the antifouling coating with antibodies.

Chapter 7 aims to place the obtained results in a broader context of potential biomedical and research applications, and provides recommendations for further research.

\subsection{References}

1. Jiang, S. Y.; Cao, Z. Q. Ultralow-Fouling, Functionalizable, and Hydrolyzable Zwitterionic Materials and Their Derivatives for Biological Applications. Adv. Mater. 2010, 22, 920-932.

2. Schlenoff, J. B. Zwitteration: Coating Surfaces with Zwitterionic Functionality to Reduce Nonspecific Adsorption. Langmuir 2014, 30, 9625-9636.

3. Ratner, B. D. Replacing and Renewing: Synthetic Materials, Biomimetics, and Tissue Engineering in Implant Dentistry. J. Dent. Educ. 2001, 65, 1340-1347. 
4. Leslie, D. C.; Waterhouse, A.; Berthet, J. B.; Valentin, T. M.; Watters, A. L.; Jain, A.; Kim, P.; Hatton, B. D.; Nedder, A.; Donovan, K.; Super, E. H.; Howell, C.; Johnson, C. P.; Vu, T. L.; Bolgen, D. E.; Rifai, S.; Hansen, A. R.; Aizenberg, M.; Super, M.; Aizenberg, J.; Ingber, D. E. A Bioinspired Omniphobic Surface Coating on Medical Devices Prevents Thrombosis and Biofouling. Nat. Biotechnol. 2014, 32, 1134-1140.

5. Iwasaki, Y.; Ishihara, K. Cell Membrane-Inspired Phospholipid Polymers for Developing Medical Devices with Excellent Biointerfaces. Sci.Technol.Adv. Mat. 2012, 13, 064101-064115.

6. Wei, Q.; Becherer, T.; Angioletti-Uberti, S.; Dzubiella, J.; Wischke, C.; Neffe, A. T.; Lendlein, A.; Ballauff, M.; Haag, R. Protein Interactions with Polymer Coatings and Biomaterials. Angew. Chem. Int. Ed. 2014, 53, 8004-8031.

7. Horbett, T. A. The Role of Adsorbed Proteins in Animal-Cell Adhesion. Colloids Surf., B 1994, 2, 225-240.

8. Chae, K. H.; Jang, Y. M.; Kim, Y. H.; Sohn, O. J.; Rhee, J. I. Anti-Fouling Epoxy Coatings for Optical Biosensor Application Based on Phosphorylcholine. Sens. Actuators, B 2007, 124, 153-160.

9. Vaisocherová, H.; Brynda, E.; Homola, J. Functionalizable Low-Fouling Coatings for Label-Free Biosensing in Complex Biological Media: Advances and Applications. Anal. Bioanal. Chem. 2015, 407, 3927-3953.

10. Blaszykowski, C.; Sheikh, S.; Thompson, M. A Survey of State-of-the-Art Surface Chemistries to Minimize Fouling from Human and Animal Biofluids. Biomater. Sci. 2015, 3, 1335-1370.

11. Li, Y.; Liu, R. Y.; Yang, J.; Shi, Y. J.; Ma, G. H.; Zhang, Z. Z.; Zhang, X. Enhanced Retention and Anti-Tumor Efficacy of Liposomes by Changing Their Cellular Uptake and Pharmacokinetics Behavior. Biomaterials 2015, 41, 1-14.

12. Ye, L.; Zhan, Y. B.; Yang, B. G.; Zhou, X.; Li, J. J.; Qin, Z. H.; Dong, D. Y.; Cui, Y. L.; Yao, F. L. Zwitterionic-Modified StarchBased Stealth Micelles for Prolonging Circulation Time and Reducing Macrophage Response. ACS Appl. Mater. Interfaces 2016, $8,4385-4398$.

13. Zheng, L. C.; Sundaram, H. S.; Wei, Z.Y.; Li, C. C.; Yuan, Z. F. Applications of Zwitterionic Polymers. React. Funct. Polym. 2017, 118, 51-61.

14. Ayres, N. Polymer Brushes: Applications in Biomaterials and Nanotechnology. Polym. Chem. 2010, 1, 769.

15. Banerjee, I.; Pangule, R. C.; Kane, R. S. Antifouling Coatings: Recent Developments in the Design of Surfaces That Prevent Fouling by Proteins, Bacteria, and Marine Organisms. Adv. Mater. 2011, 23, 690-718.

16. Rodriguez-Emmenegger, C.; Houska, M.; Alles, A. B.; Brynda, E. Surfaces Resistant to Fouling from Biological Fluids: Towards Bioactive Surfaces for Real Applications. Macromol. Biosci. 2012, 12, 1413-1422.

17. Chen, S. F.; Li, L. Y.; Zhao, C.; Zheng, J. Surface Hydration: Principles and Applications toward Low-Fouling/Nonfouling Biomaterials. Polymer 2010, 51, 5283-5293.

18. Blaszykowski, C.; Sheikh, S.; Thompson, M. Surface Chemistry to Minimize Fouling from Blood-Based Fluids. Chem. Soc. Rev. 2012, 41, 5599-5612.

19. Ostuni, E.; Chapman, R. G.; Holmlin, R. E.;Takayama, S.; Whitesides, G. M.A Survey of Structure-Property Relationships of Surfaces That Resist the Adsorption of Protein. Langmuir 2001, 17, 5605-5620.

20. Pochechueva, T.; Chinarev, A.; Bovin, N.; Fedier, A.; Jacob, F.; Heinzelmann-Schwarz, V. Pegylation of Microbead Surfaces Reduces Unspecific Antibody Binding in Glycan-Based Suspension Array. J. Immunol. Methods 2014, 412, 42-52.

21. Riedel, T.; Riedelova-Reicheltova, Z.; Majek, P.; Rodriguez-Emmenegger, C.; Houska, M.; Dyr, J. E.; Brynda, E. Complete Identification of Proteins Responsible for Human Blood Plasma Fouling on Poly(Ethylene Glycol)-Based Surfaces. Langmuir 2013, 29, 3388-3397.

22. Li, L. Y.; Chen, S. F.; Zheng, J.; Ratner, B. D.; Jiang, S. Y. Protein Adsorption on Oligo(Ethylene Glycol)-Terminated Alkanethiolate Self-Assembled Monolayers: The Molecular Basis for Nonfouling Behavior. J. Phys. Chem. B 2005, 109, $2934-$ 2941.

23. Alswieleh, A. M.; Cheng, N.; Canton, I.; Ustbas, B.; Xue, X.; Ladmiral, V.; Xia, S.; Ducker, R. E.; El Zubir, O.; Cartron, M. L.; Hunter, C. N.; Leggett, G. J.; Armes, S. P. Zwitterionic Poly(Amino Acid Methacrylate) Brushes. J. Am. Chem. Soc. 2014, 136, 9404-9413. 
24. McArthur, S. L.; McLean, K. M.; Kingshott, P.; St John, H. A. W.; Chatelier, R. C.; Griesser, H. J. Effect of Polysaccharide Structure on Protein Adsorption. Colloids Surf. B Biointerfaces 2000, 17, 37-48.

25. Chen, H.; Zhao, C.; Zhang, M. Z.; Chen, Q.; Ma, J.; Zheng, J. Molecular Understanding and Structural-Based Design of Polyacrylamides and Polyacrylates as Antifouling Materials. Langmuir 2016, 32, 3315-3330.

26. Vaisocherova, H.; Zhang, Z.; Yang, W.; Cao, Z. Q.; Cheng, G.; Taylor, A. D.; Piliarik, M.; Homola, J.; Jiang, S.Y. Functionalizable Surface Platform with Reduced Nonspecific Protein Adsorption from Full Blood Plasma-Material Selection and Protein Immobilization Optimization. Biosens. Bioelectron. 2009, 24, 1924-1930.

27. Ladd, J.; Zhang, Z.; Chen, S.; Hower, J. C.; Jiang, S. Zwitterionic Polymers Exhibiting High Resistance to Nonspecific Protein Adsorption from Human Serum and Plasma. Biomacromolecules 2008, 9, 1357-1361.

28. Lee, S.-W.; Laibinis, P. E. Protein-Resistant Coatings for Glass and Metal Oxide Surfaces Derived from Oligo(Ethylene Glycol)-Terminated Alkyltrichlorosilanes. Biomaterials 1998, 19, 1669-1675.

29. Lange, S. C.; van Andel, E.; Smulders, M. M. J.; Zuilhof, H. Efficient and Tunable Three-Dimensional Functionalization of Fully Zwitterionic Antifouling Surface Coatings. Langmuir 2016, 32, 10199-10205.

30. Nguyen, A.T.; Baggerman, J.; Paulusse, J. M. J.; van Rijn, C. J. M.; Zuilhof, H. Stable Protein-Repellent Zwitterionic Polymer Brushes Grafted from Silicon Nitride. Langmuir 2011, 27, 2587-2594.

31. Prime, K. L.; Whitesides, G. M. Self-Assembled Organic Monolayers - Model Systems for Studying Adsorption of Proteins at Surfaces. Science 1991, 252, 1164-1167.

32. Chapman, R. G.; Ostuni, E.; Takayama, S.; Holmlin, R. E.; Yan, L.; Whitesides, G. M. Surveying for Surfaces That Resist the Adsorption of Proteins. J.Am. Chem. Soc. 2000, 122, 8303-8304.

33. Rodriguez-Emmenegger, C.; Brynda, E.; Riedel, T.; Houska, M.; Subr, V.; Alles, A. B.; Hasan, E.; Gautrot, J. E.; Huck, W.T. S. Polymer Brushes Showing Non-Fouling in Blood Plasma Challenge the Currently Accepted Design of Protein Resistant Surfaces. Macromol. Rapid Commun. 2011, 32, 952-957.

34. Holland, N. B.; Qiu, Y. X.; Ruegsegger, M.; Marchant, R. E. Biomimetic Engineering of Non-Adhesive Glycocalyx-Like Surfaces Using Oligosaccharide Surfactant Polymers. Nature 1998, 392, 799-801.

35. Karakoti, A. S.; Das, S.; Thevuthasan, S.; Seal, S. PEGylated Inorganic Nanoparticles. Angew. Chem. Int. Ed. 201 1, 50, $1980-1994$.

36. Lowe, S.; O’Brien-Simpson, N. M.; Connal, L. A. Antibiofouling Polymer Interfaces: Poly(Ethylene Glycol) and Other Promising Candidates. Polym. Chem. 2015, 6, 198-212.

37. Suk, J. S.; Xu, Q. G.; Kim, N.; Hanes, J.; Ensign, L. M. Pegylation as a Strategy for Improving Nanoparticle-Based Drug and Gene Delivery. Adv. Drug Delivery Rev. 2016, 99, 28-51.

38. Li, L. Y.; Chen, S. F.; Jiang, S.Y. Protein Interactions with Oligo(Ethylene Glycol) (OEG) Self-Assembled Monolayers: OEG Stability, Surface Packing Density and Protein Adsorption. J. Biomater. Sci., Polym. Ed. 2007, 18, 1415-1427.

39. Kawai, F. Microbial Degradation of Polyethers. Appl. Microbiol. Biotechnol. 2002, 58, 30-38.

40. Armstrong, J. K.; Hempel, G.; Koling, S.; Chan, L. S.; Fisher,T.; Meiselman, H. J.; Garratty, G. Antibody against Poly(Ethylene Glycol) Adversely Affects Peg-Asparaginase Therapy in Acute Lymphoblastic Leukemia Patients. Cancer 2007, 110, $103-111$.

41. Schellekens, H.; Hennink, W. E.; Brinks, V.The Immunogenicity of Polyethylene Glycol: Facts and Fiction. Pharm. Res. 2013, 30, 1729-1734.

42. Kim, J. C.; Kim, M.; Jung, J.; Kim, H.; Kim, I. J.; Kim, J. R.; Ree, M. Biocompatible Characteristics of Sulfobetaine-Containing Brush Polymers. Macromol. Res. 2012, 20, 746-753.

43. Leng, C.; Sun, S. W.; Zhang, K. X.; Jiang, S.Y.; Chen, Z. Molecular Level Studies on Interfacial Hydration of Zwitterionic and Other Antifouling Polymers in Situ. Acta Biomater. 2016, 40, 6-15.

44. Leng, C.; Hung, H. C.; Sun, S. W.; Wang, D. Y.; Li, Y. T.; Jiang, S. Y.; Chen, Z. Probing the Surface Hydration of Nonfouling Zwitterionic and Peg Materials in Contact with Proteins. ACS Appl. Mater. Interfaces 2015, 7, 16881-16888.

45. Virtanen, J. A.; Cheng, K. H.; Somerharju, P. Phospholipid Composition of the Mammalian Red Cell Membrane Can Be Rationalized by a Superlattice Model. Proc. Natl.Acad. Sci. U. S.A. 1998, 95, 4964-4969. 
46. Kadoma, Y.; Nakabayashi, N.; Masuhara, E.; Yamauchi, J. Synthesis and Hemolysis Test of the Polymer Containing Phosphorylcholine Groups. Kobunshi Ronbunshu 1978, 35, 423-427.

47. Ueda, T.; Oshida, H.; Kurita, K.; Ishihara, K.; Nakabayashi, N. Preparation of 2-Methacryloyloxyethyl Phosphorylcholine Copolymers with Alkyl Methacrylates and Their Blood Compatibility. Polym.J. 1992, 24, 1259-1269.

48. Ishihara, K.; Ueda, T.; Nakabayashi, N. Preparation of Phospholipid Polylners and Their Properties as Polymer Hydrogel Membranes. Polym.J. 1990, 22, 355-360.

49. Zhang, Z.; Vaisocherova, H.; Cheng, G.; Yang, W.; Xue, H.; Jiang, S.Y. Nonfouling Behavior of Polycarboxybetaine-Grafted Surfaces: Structural and Environmental Effects. Biomacromolecules 2008, 9, 2686-2692.

50. Ning, J.; Li, G.; Haraguchi, K. Synthesis of Highly Stretchable, Mechanically Tough, Zwitterionic Sulfobetaine Nanocomposite Gels with Controlled Thermosensitivities. Macromolecules 2013, 46, 5317-5328.

51. Sin, M. C.; Chen, S. H.; Chang, Y. Hemocompatibility of Zwitterionic Interfaces and Membranes. Polym. J. 2014, 46, 436443.

52. Yang, W.; Zhang, L.; Wang, S. L.; White, A. D.; Jiang, S. Y. Functionalizable and Ultra Stable Nanoparticles Coated with Zwitterionic Poly(Carboxybetaine) in Undiluted Blood Serum. Biomaterials 2009, 30, 5617-5621.

53. Zhang, Z.; Chao, T.; Chen, S. F.; Jiang, S. Y. Superlow Fouling Sulfobetaine and Carboxybetaine Polymers on Glass Slides. Langmuir 2006, 22, 10072-10077.

54. Chou,Y. N.;Wen,T. C.; Chang,Y. Zwitterionic Surface Grafting of Epoxylated Sulfobetaine Copolymers for the Development of Stealth Biomaterial Interfaces. Acta Biomater. 2016, 40, 78-91.

55. Lin, W. F.; Ma, G. L.; Wu, J.; Chen, S. F. Different in Vitro and in Vivo Behaviors between Poly(Carboxybetaine Methacrylate) and Poly(Sulfobetaine Methacrylate). Colloids Surf., B 2016, 146, 888-894.

56. Yang, W.; Chen, S. F.; Cheng, G.; Vaisocherova, H.; Xue, H.; Li, W.; Zhang, J. L.; Jiang, S. Y. Film Thickness Dependence of Protein Adsorption from Blood Serum and Plasma onto Poly(Sulfobetaine)-Grafted Surfaces. Langmuir 2008, 24, 9211 9214.

57. Huxtable, R. J. Physiological Actions of Taurine. Physiol. Rev. 1992, 72, 101-163.

58. Stadmiller, S. S.; Gorensek-Benitez, A. H.; Guseman, A. J.; Pielak, G. J. Osmotic Shock Induced Protein Destabilization in Living Cells and Its Reversal by Glycine Betaine. J. Mol. Biol. 2017, 429, 1155-1161.

59. Krishnamoorthy, M.; Hakobyan, S.; Ramstedt, M.; Gautrot, J. E. Surface-Initiated Polymer Brushes in the Biomedical Field: Applications in Membrane Science, Biosensing, Cell Culture, Regenerative Medicine and Antibacterial Coatings. Chem. Rev. 2014, 114, 10976-11026.

60. Yang, W.; Xue, H.; Carr, L. R.; Wang, J.; Jiang, S. Zwitterionic Poly(Carboxybetaine) Hydrogels for Glucose Biosensors in Complex Media. Biosens. Bioelectron. 2011, 26, 2454-2459.

61. von Muhlen, M. G.; Brault, N. D.; Knudsen, S. M.; Jiang, S. Y.; Manalis, S. R. Label-Free Biomarker Sensing in Undiluted Serum with Suspended Microchannel Resonators. Anal. Chem. 2010, 82, 1905-1910.

62. Lin, W. F.; Ma, G. L.; Kampf, N.; Yuan, Z. F.; Chen, S. F. Development of Long-Circulating Zwitterionic Cross-Linked Micelles for Active-Targeted Drug Delivery. Biomacromolecules 2016, 17, 2010-2018.

63. Bengani-Lutz, P.; Converse, E.; Cebe, P.; Asatekin, A. Self-Assembling Zwitterionic Copolymers as Membrane Selective Layers with Excellent Fouling Resistance: Effect of Zwitterion Chemistry. ACS Appl. Mater. Interfaces 2017, 9, 20859-20872.

64. Xiang, T.; Lu, T.; Xie, Y.; Zhao, W. F.; Sun, S. D.; Zhao, C. S. Zwitterionic Polymer Functionalization of Polysulfone Membrane with Improved Antifouling Property and Blood Compatibility by Combination of ATRP and Click Chemistry. Acta Biomater. 2016, 40, 162-171.

65. Mi, L.; Jiang, S. Integrated Antimicrobial and Nonfouling Zwitterionic Polymers. Angew. Chem. Int. Ed. 2014, 53, $1746-54$.

66. Zhang, Z.; Finlay, J. A.; Wang, L. F.; Gao, Y.; Callow, J. A.; Callow, M. E.; Jiang, S. Y. Polysulfobetaine-Grafted Surfaces as Environmentally Benign Ultralow Fouling Marine Coatings. Langmuir 2009, 25, 13516-13521.

67. Kirk, J. T.; Brault, N. D.; Baehr-Jones, T.; Hochberg, M.; Jiang, S.; Ratner, D. M. Zwitterionic Polymer-Modified Silicon 
Microring Resonators for Label-Free Biosensing in Undiluted Human Plasma. Biosens. Bioelectron. 2013, 42, 100-105.

68. Lisalova, H.; Brynda, E.; Houska, M.; Visova, I.; Mrkvova, K.; Song, X. C.; Gedeonova, E.; Surman, F.; Riedel, T.; PopGeorgievski, O.; Homola, J. Ultralow-Fouling Behavior of Biorecognition Coatings Based on Carboxy-Functional Brushes of Zwitterionic Homo- and Copolymers in Blood Plasma: Functionalization Matters. Anal. Chem. 2017, 89, 3524-3531.

69. Shao, Q.; Jiang, S.Y. Molecular Understanding and Design of Zwitterionic Materials. Adv. Mater. 2015, 27, 15 -26.

70. Higaki,Y.; Inutsuka,Y.; Sakamaki,T.; Terayama,Y.; Takenaka, A.; Higaki, K.; Yamada, N. L.; Moriwaki,T.; Ikemoto, Y.; Takahara, A. Effect of Charged Group Spacer Length on Hydration State in Zwitterionic Poly(Sulfobetaine) Brushes. Langmuir 2017, 33, 8404-8412.

71. Shao, Q.; He, Y.; White, A. D.; Jiang, S.Y. Difference in Hydration between Carboxybetaine and Sulfobetaine. J. Phys. Chem. B 2010, 114, 16625-16631.

72. Wang, T.; Kou, R.; Liu, H.; Liu, L.; Zhang, G.; Liu, G. Anion Specificity of Polyzwitterionic Brushes with Different Carbon Spacer Lengths and Its Application for Controlling Protein Adsorption. Langmuir 2016, 32, 2698-2707.

73. Vaisocherova, H.; Yang, W.; Zhang, Z.; Cao, Z. Q.; Cheng, G.; Piliarik, M.; Homola, J.; Jiang, S. Y. Ultralow Fouling and Functionalizable Surface Chemistry Based on a Zwitterionic Polymer Enabling Sensitive and Specific Protein Detection in Undiluted Blood Plasma. Anal. Chem. 2008, 80, 7894-7901.

74. Choi, S.; Choi, B. C.; Xue, C.; Leckband, D. Protein Adsorption Mechanisms Determine the Efficiency of Thermally Controlled Cell Adhesion on Poly(N-Isopropyl Acrylamide) Brushes. Biomacromolecules 2013, 14, 92-100.

75. Yang, W. F.; Zhang, R.;Wu,Y.; Pei, X. W.; Liu, Y. P.; Zhou, F. Enhancement of Graft Density and Chain Length of Hydrophilic Polymer Brush for Effective Marine Antifouling. J.Appl. Polym. Sci. 2018, 135, 46232.

76. Dalsin, J. L.; Messersmith, P. B. Bioinspired Antifouling Polymers. Mater.Today 2005, 8, 38-46.

77. Zoppe, J. O.; Ataman, N. C.; Mocny, P.; Wang, J.; Moraes, J.; Klok, H. A. Surface-Initiated Controlled Radical Polymerization: State-of-the-Art, Opportunities, and Challenges in Surface and Interface Engineering with Polymer Brushes. Chem. Rev. 2017, 117, 1105-1318.

78. Matyjaszewski, K.; Tsarevsky, N. V. Macromolecular Engineering by Atom Transfer Radical Polymerization. J.Am. Chem. Soc. 2014, 136, 6513-6533.

79. Zhang, J.; Kou, R.; Liu, G. M. Effect of Salt Concentration on the pH Responses of Strong and Weak Polyelectrolyte Brushes. Langmuir 2017, 33, 6838-6845.

80. Mi, L.; Bernards, M. T.; Cheng, G.; Yu, Q.; Jiang, S. Ph Responsive Properties of Non-Fouling Mixed-Charge Polymer Brushes Based on Quaternary Amine and Carboxylic Acid Monomers. Biomaterials 2010, 31, 2919-2925.

81. Zhang,Z.; Moxey, M.;Alswieleh,A.; Morse,A.J.; Lewis,A.L.; Geoghegan, M.; Leggett, G.J. Effect of Salt on PhosphorylcholineBased Zwitterionic Polymer Brushes. Langmuir 2016, 32, 5048-5057.

82. Pereira, A. D.; Rodriguez-Emmenegger, C.; Surman, F.; Riedel, T.; Alles, A. B.; Brynda, E. Use of Pooled Blood Plasmas in the Assessment of Fouling Resistance. RSC Adv. 2014, 4, 2318-2321.

83. Matyjaszewski, K. Atom Transfer Radical Polymerization (ATRP): Current Status and Future Perspectives. Macromolecules 2012, 45, 4015-4039.

84. He, W.; Jiang, H.; Zhang, L.; Cheng, Z.; Zhu, X. Atom Transfer Radical Polymerization of Hydrophilic Monomers and Its Applications. Polym. Chem. 2013, 4, 2919-2938.

85. Boyer, C.; Corrigan, N.A.; Jung, K.; Nguyen, D.; Nguyen,T.-K.; Adnan, N. N. M.; Oliver, S.; Shanmugam, S.; Yeow, J. CopperMediated Living Radical Polymerization (AtomTransfer Radical Polymerization and Copper(0) Mediated Polymerization): From Fundamentals to Bioapplications. Chem. Rev. 2016, 116, 1803-1949.

86. Wang, J.-S.; Matyjaszewski, K. Controlled/”Living” Radical Polymerization. Halogen Atom Transfer Radical Polymerization Promoted by a Cu(I)/Cu(II) Redox Process. Macromolecules 1995, 28, 7901-7910.

87. Wang, J.-S.; Matyjaszewski, K. Controlled/”Living” Radical Polymerization. Atom Transfer Radical Polymerization in the Presence of Transition-Metal Complexes. J.Am. Chem. Soc. 1995, 117, 5614-5615. 
88. Kato, M.; Kamigaito, M.; Sawamoto, M.; Higashimura, T. Polymerization of Methyl Methacrylate with the Carbon Tetrachloride/Dichlorotris- (Triphenylphosphine)Ruthenium(II)/Methylaluminum Bis(2,6-Di-Tert-Butylphenoxide) Initiating System: Possibility of Living Radical Polymerization. Macromolecules 1995, 28, 1721-1723.

89. Matyjaszewski, K.; Jakubowski, W.; Min, K.; Tang, W.; Huang, J. Y.; Braunecker, W. A.; Tsarevsky, N. V. Diminishing Catalyst Concentration in Atom Transfer Radical Polymerization with Reducing Agents. Proc. Natl.Acad. Sci. U. S. A. 2006, 103, 1530915314.

90. Li, B.;Yu, B.; Ye, Q.; Zhou, F. Tapping the Potential of Polymer Brushes through Synthesis. Acc. Chem. Res. 2014, 48, $229-237$.

91. Zhang, P.; Sun, F.; Tsao, C.; Liu, S. J.; Jain, P.; Sinclair, A.; Hung, H. C.; Bai,T.;Wu, K.; Jiang, S.Y. Zwitterionic Gel Encapsulation Promotes Protein Stability, Enhances Pharmacokinetics, and Reduces Immunogenicity. Proc. Natl. Acad. Sci. U. S. A. 2015, 112, 12046-12051.

92. Keefe, A. J.; Jiang, S. Poly(Zwitterionic)Protein Conjugates Offer Increased Stability without Sacrificing Binding Affinity or Bioactivity. Nat. Chem. 2011, 4, 59-63.

93. Pujari, S. P.; Scheres, L.; Marcelis, A. T. M.; Zuilhof, H. Covalent Surface Modification of Oxide Surfaces. Angew. Chem. Int. Ed. 2014, 53, 6322-6356.

94. Kim, G.; Yong, Y.; Kang, H. J.; Park, K.; Kim, S. I.; Lee, M.; Huh, N. Zwitterionic Polymer-Coated Immunobeads for BloodBased Cancer Diagnostics. Biomaterials 2014, 35, 294-303.

95. Lin, R.; Li, Y. C.; MacDonald, T.; Wu, H.; Provenzale, J.; Peng, X. G.; Huang, J.; Wang, L. Y.; Wang, A. Y.; Yang, J.Y.; Mao, H. Improving Sensitivity and Specificity of Capturing and Detecting Targeted Cancer Cells with Anti-Biofouling Polymer Coated Magnetic Iron Oxide Nanoparticles. Colloids Surf., B 2017, 150, 261-270.

96. Gonzalez-Guerrero, A. B.; Maldonado, J.; Herranz, S.; Lechuga, L. M. Trends in Photonic Lab-on-Chip Interferometric Biosensors for Point-of-Care Diagnostics. Anal. Meth. 2016, 8, 8380-8394.

97. Brault, N. D.; White, A. D.; Taylor, A. D.; Yu, Q. M.; Jiang, S.Y. Directly Functionalizable Surface Platform for Protein Arrays in Undiluted Human Blood Plasma. Anal. Chem. 2013, 85, 1447-1453.

98. Hu, G. J.; Emrick, T. Functional Choline Phosphate Polymers. J.Am. Chem. Soc. 2016, 138, 1828-1831.

99. Huang, C. J.; Li, Y.; Jiang, S. Zwitterionic Polymer-Based Platform with Two-Layer Architecture for Ultra Low Fouling and High Protein Loading. Anal. Chem. 2012, 84, 3440-3445.

100. Song, L. J.; Zhao, J.; Luan, S. F.; Ma, J.; Liu, J. C.; Xu, X. D.; Yin, J. H. Fabrication of a Detection Platform with Boronic-AcidContaining Zwitterionic Polymer Brush. ACS Appl. Mater. Interfaces 2013, 5, 13207-13215.

101.Guo, X. Surface Plasmon Resonance Based Biosensor Technique: A Review. J.Biophotonics 2012, 5, 483-501.

102.Heideman, R.; Hoekman, M.; Schreuder, E. Triplex-Based Integrated Optical Ring Resonators for Lab-on-a-Chip and Environmental Detection. IEEE J. Sel.Top. Quant. 2012, 18, 1583-1596.

103.Ramachandran, A.; Wang, S.; Clarke, J.; Ja, S. J.; Goad, D.; Wald, L.; Flood, E. M.; Knobbe, E.; Hryniewicz, J. V.; Chu, S. T.; Gill, D.; Chen, W.; King, O.; Little, B. E. A Universal Biosensing Platform Based on Optical Micro-Ring Resonators. Biosens. Bioelectron. 2008, 23, 939-944.

104.Ren, K. N.; Zhou, J. H.;Wu, H. K. Materials for Microfluidic Chip Fabrication. Acc. Chem. Res. 2013, 46, 2396-2406.

105. Bhairamadgi, N. S.; Pujari, S. P.; Trovela, F. G.; Debrassi, A.; Khamis, A. A.; Alonso, J. M.; Al Zahrani, A. A.; Wennekes, T.; AlTuraif, H. A.; van Rijn, C.; Alhamed, Y. A.; Zuilhof, H. Hydrolytic and Thermal Stability of Organic Mono Layers on Various Inorganic Substrates. Langmuir 2014, 30, 5829-5839.

106. Scheres, L.; Giesbers, M.; Zuilhof, H. Organic Monolayers onto Oxide-Free Silicon with Improved Surface Coverage: Alkynes Versus Alkenes. Langmuir 2010, 26, 4790-4795.

107.Joshi, S.; Pellacani, P.; van Beek, T. A.; Zuilhof, H.; Nielen, M. W. F. Surface Characterization and Antifouling Properties of Nanostructured Gold Chips for Imaging Surface Plasmon Resonance Biosensing. Sens. Actuators, B 2015, 209, 505-514.

108. Borozenko, O.; Machado, V.; Skene, W. G.; Giasson, S. Organophosphonic Acids as Viable Linkers for the Covalent Attachment of Polyelectrolyte Brushes on Silica and Mica Surfaces. Polym. Chem. 2014, 5, 5740-5750. 
109.Rosso, M.; Nguyen, A.T.; de Jong, E.; Baggerman, J.; Paulusse, J. M. J.; Giesbers, M.; Fokkink, R. G.; Norde, W.; Schroen, K.; van Rijn, C. J. M.; Zuilhof, H. Protein-Repellent Silicon Nitride Surfaces: UV-Induced Formation of Oligoethylene Oxide Mono Layers. ACS Appl. Mater. Interfaces 2011, 3, 697-704.

110.Reddy, L. H.; Arias, J. L.; Nicolas, J.; Couvreur, P. Magnetic Nanoparticles: Design and Characterization, Toxicity and Biocompatibility, Pharmaceutical and Biomedical Applications. Chem. Rev. 2012, 112, 5818-5878.

111. Haukanes, B. I.; Kvam, C. Application of Magnetic Beads in Bioassays. Biotechnology 1993, 11, 60-63.

112.Nguyen, A. T.; Baggerman, J.; Paulusse, J. M. J.; Zuilhof, H.; van Rijn, C. J. M. Bioconjugation of Protein-Repellent Zwitterionic Polymer Brushes Grafted from Silicon Nitride. Langmuir 2012, 28, 604-610.

113.Li, Y.; Giesbers, M.; Gerth, M.; Zuilhof, H. Generic Top-Functionalization of Patterned Antifouling Zwitterionic Polymers on Indium Tin Oxide. Langmuir 2012, 28, 12509-12517.

114.Lee, B. S.; Lee, J. K.; Kim, W. J.; Jung,Y. H.; Sim, S. J.; Lee, J.; Choi, I. S. Surface-Initiated, Atom Transfer Radical Polymerization of Oligo(Ethylene Glycol) Methyl Ether Methacrylate and Subsequent Click Chemistry for Bioconjugation. Biomacromolecules 2007, 8, 744-749.

115. Kolb, H. C.; Finn, M. G.; Sharpless, K. B. Click Chemistry: Diverse Chemical Function from a Few Good Reactions. Angew. Chem. Int. Ed. 2001, 40, 2004-2021.

116.Spiliopoulos, N.; Koutsioubas, A. G.; Anastassopoulos, D. L.; Vradis, A. A.; Toprakcioglu, C.; Menelle, A.; Mountrichas, G.; Pispas, S. Neutron Reflectivity Study of Free-End Distribution in Polymer Brushes. Macromolecules 2009, 42, 6209-6214.

117.Sivashanmugan, K.; Liu, P.-C.; Tsai, K.-W.; Chou, Y.-N.; Lin, C.-H.; Chang, Y.; Wen, T.-C. An Anti-Fouling Nanoplasmonic Sers Substrate for Trapping and Releasing a Cationic Fluorescent Tag from Human Blood Solution. Nanoscale 2017, 9, 2865 2874. 


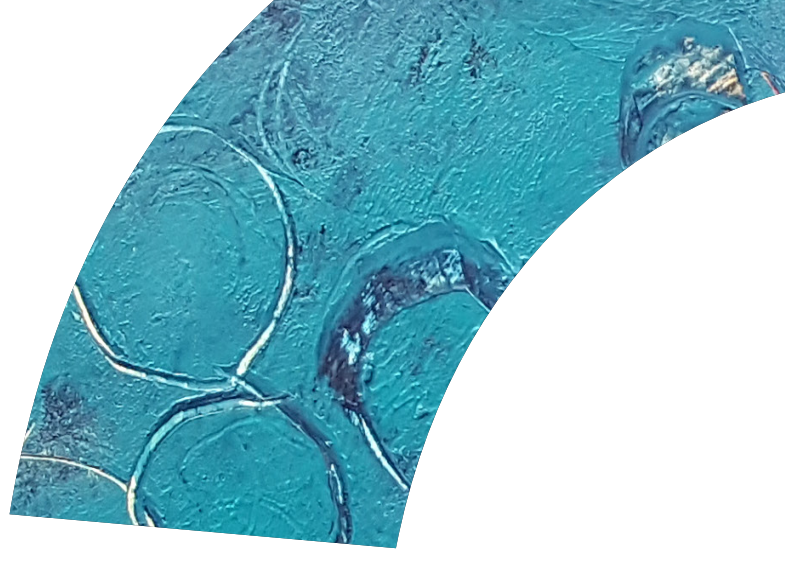

\section{Chapter.}




\section{Efficient}

and Tunable

3D-Functionalization of Fully Zwitterionic Antifouling Surface Coatings

Lange, S. C.; van Andel, E.; Smulders, M. M. J.; Zuilhof, H. Efficient and Tunable ThreeDimensional Functionalization of Fully Zwitterionic Antifouling Surface Coatings. Langmuir 2016, 32, 10199-10205. 


\begin{abstract}
In order to enhance the sensitivity and selectivity of surface-based (bio)sensors, it is of crucial importance to diminish background signals that arise from the non-specific binding of biomolecules, so-called biofouling. Zwitterionic polymer brushes have been shown to be excellent antifouling materials. However, for sensing purposes, antifouling does no suffice, but needs to be combined with the possibility to efficiently modify the brush with recognition units. So far this has only been achieved at the expense of either antifouling properties or binding capacity. Herein we present a conceptually new approach by integrating both characteristics into a single, tailor-made monomer: a novel sulfobetainebased zwitterionic monomer equipped with a clickable azide moiety. Copolymerization of this monomer with a well-established standard sulfobetaine monomer results in highly antifouling surface coatings with a high, yet tunable amount of clickable groups present throughout the entire brush. Subsequent functionalization of the azido-brushes, via e.g. widely used strain-promoted alkyne azide click reactions yields fully zwitterionic 3D-functionalized coatings with a recognition unit of choice that can be tailored for any specific application. Here we show a proof of principle with biotin-functionalized brushes on $\mathrm{Si}_{3} \mathrm{~N}_{4}$ that combine excellent antifouling properties with specific avidin binding from a protein mixture. The signal-to-noise ratio is significantly improved over traditional chain end modification of sulfobetaine polymer brushes, even if the azide content is lowered to $1 \%$. This therefore offers a viable approach for the development of biosensors with greatly enhanced performance.
\end{abstract}




\subsection{Introduction}

Non-specific protein absorption, one of the first stages of (bio)fouling, is a recurring problem in various fields, such as in biomedical and bioanalytical applications, since it strongly reduces the sensitivity for the detection of specific targets from complex mixtures. ${ }^{1}$ In order to prevent (bio)fouling, antifouling surface coatings are extensively used to minimize non-specific interactions between (bio)molecules and the substrate. The antifouling capability of coated surfaces is mainly determined by the internal architecture ${ }^{2}$ and the resulting hydration of the used polymer coating. Due to their electrostatically induced hydration layer, zwitterionic polymeric materials made of carboxy- $(\mathrm{CB})^{3}$ and sulfobetaine $(\mathrm{SB})^{4-5}$ monomers perform extremely well under full-blood and other physiological conditions, ${ }^{6-8}$ and have thus been recognized as high-performance antifouling materials. ${ }^{9-10}$ Progress in polymerization methods, in particular surface-initiated atom transfer radical polymerization (SI-ATRP), ${ }^{11-14}$ has provided access to robust and versatile substrate coatings with full control over brush growth. Choosing the appropriate monomers for ATRP enables tailoring of surface properties including antifouling abilities, (bio)compatibility and (bio)molecular recognition. Functionalized antifouling zwitterion-based polymer brush coatings have been utilized extensively for specific (bio)molecule immobilization, e.g. in the context of sensing. ${ }^{15}$ This approach requires the presence of recognition units within the surface coatings, and various methods for selective brush modification have been reported. For example, poly(carboxybetaine) brushes (pCB) can be functionalized using the random coupling of their intrinsic carboxylate groups with biospecific groups via EDC/NHS chemistry. ${ }^{16,17}$ However, modification via the carboxyl groups occurs at the expense of electrical neutrality of the polymer brush, which thus diminishes its antifouling character.

Furthermore, the antifouling properties of carboxybetaine polymer brushes are sensitive to $\mathrm{pH}$ changes, ${ }^{18}$ due to protonation of the carboxyl functions at low $\mathrm{pH}$ values. These drawbacks brought the usage of the highly stable, but chemically inert sulfobetaine monomers into focus. ${ }^{4}$ Lacking intrinsic functionality, modification of sulfobetaine polymer brushes (pSB) has been achieved by block copolymer structures. ${ }^{19}$ For example, Ohno and co-workers, constructed first a zwitterionic pSB layer, onto which a second layer was grown using NHS-modified nonzwitterionic monomers. ${ }^{20}$ In an alternative approach by Yin, ${ }^{21}$ functionality was introduced into pSB brushes via copolymerization of non-zwitterionic glycidylmethacrylate (GMA) monomers. In this way, addressable epoxide moieties were incorporated into grafted sulfobetaine polymer chains. Although showing potential as functional antifouling coatings, the introduction of (10\% or 50\%) non-zwitterionic epoxide monomers diminished the antifouling performance. Another approach was shown by our lab via post-modification of fully zwitterionic sulfobetaine brushes through top exchange reactions of the remaining terminal halogen substituent after ATRP. ${ }^{22-23}$ While effective, this approach is limited to relatively thin brushes $(20-25 \mathrm{~nm})$, as in thicker brushes the end groups become buried inside the brush, ${ }^{24}$ thus rendering them unavailable for functionalization. In addition, top functionalization allows only for a limited number of newly introduced anchor points. 
To overcome the limitations outlined above, we present a conceptually new approach that allows for efficient three-dimensional polymer brush functionalization, without compromising the zwitterionic character, and thereby fully preserving the antifouling properties of the resulting coating. To this end, we combine functionality and zwitterionic properties in one single sulfobetaine-based monomer. Herein we present the efficient synthesis of such clickable monomer (5) and its subsequent copolymerization with a standard SB monomer using SI-ATRP (see Figure 2.1, Scheme 2.1). As representative substrate we selected stoichiometric silicon nitride $\left(\mathrm{Si}_{3} \mathrm{~N}_{4}\right)$, as this is an often-used substrate for optofluidic devices such as micro-ring resonator-based sensing systems. ${ }^{25-27}$ For this purpose, an efficient and scalable three-step route towards amine-terminated $\mathrm{Si}_{3} \mathrm{~N}_{4}$ substrates is also presented, which enables access to surfaceinitiated ATRP. The number of functional groups within the three-dimensional structure of the brushes can be straightforwardly tuned by control over the monomer ratio. These functional groups are fully addressable for subsequent post-modification with recognition units of interest, e.g. via the highly effective Strain-Promoted Azide Alkyne Click (SPAAC) reaction. ${ }^{23,}$ ${ }^{28-32}$ In this study, the detailed characterization of the brush composition and modification is firstly shown by introduction of a fluorine-labeled tag. In addition, as a proof of principle, functionalization with a biotin recognition unit and exposure to streptavidin-containing protein mixtures revealed excellent antifouling properties combined with highly efficient and specific avidin binding.

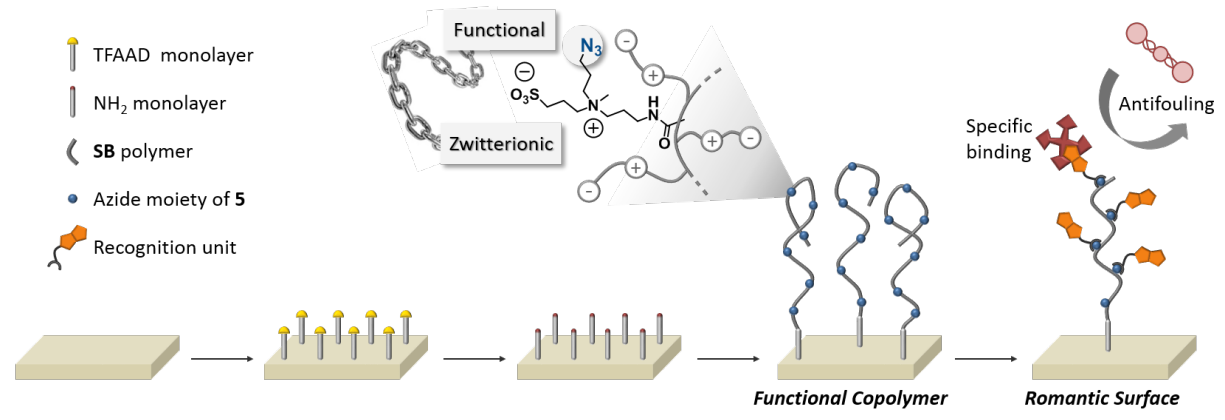

Figure 2.1 Schematic representation of functional antifouling polymer brushes as obtained from copolymerization of clickable monomer 5 with a standard SB monomer (see Scheme 2.1).

\subsection{Material and methods}

\subsubsection{Materials}

All chemicals and solvents were purchased from commercial sources and used without further purification. Tetrabutylammonium bromide $\geq 98 \%, 10$-undecenoyl chloride $\geq 97 \%$, sodium azide (BioUltra) $\geq 99.5 \%$, N-1-(3-aminopropyl)-N-1-methylpropane-1,3-diamine, sulforyl 
chloride $\geq 97 \%$, potassium carbonate, copper sulfate $\cdot 5 \mathrm{H}_{2} \mathrm{O}, 3$-bromopropanesulfonic acid sodium salt $\geq 97 \%$, dioxane $99.8 \%$, Hydroxybenzotriazole $\geq 97 \%$ (HOBt), methacrylic acid (with 250 ppm MEHQ as inhibitor) 99\%, ethanol $\geq 99.8$ Chromasolv $^{\circledR}$ absolute for HPLC, $\mathrm{N}$-(3-(dimethylamino)propyl)methacrylamide 99\% (DMAPMA), N,N-diisopropylethylamine (DIPEA), copper(I)chloride $\geq 99 \%$, copper(II) chloride 97\%, 4-methylmorpholine 99\% and 2,2'-bipyridine 99\% (Bpy), acetone (semiconductor grade, VLSI PURANALTM Honeywell 17617), sodium hydroxide $\geq 98 \%$ (anhydrous pellets), sodium chloride $\geq 99.5 \%$ (BioXtra), sodium phosphate dibasic (anhydrous), hydrofluoric acid (40\% puriss. p.a.) and fibrinogen (fraction I from porcine plasma, 78\% protein) were purchased from SigmaAldrich. Trifluoroacetic acid 99\% extra pure, di-tert-butyl dicarbonate 97\%, imidazole $99 \%$, ethyl acetate $>99 \%$, sulfuric acid $96 \%$ extra pure, hydrochloric acid $37 \%$ solution in water, dimethyl formamide $99.8 \%$ for peptide synthesis, and methyl sulfoxide $99.8 \%$, were purchased from Acros Organics. Other chemicals were ordered as follows: dichloromethane (DCM) and avidin (egg white) (VWR International S.A.S.), Silica (Silicycle), TLC silica gel $60 \mathrm{~F}_{254}$, potassium chloride (EMPURE®), and potassium dihydrogen phosphate (Merck), 1-ethyl-3-(3-dimethylaminopropyl)carbodiimide $\cdot \mathrm{HCl}$ (Novabiochem), methanol HPLC grade $(\mathrm{MeOH})$ (Rathburn Chemicals Ltd.), isopropanol 99.8\% HPLC grade (Biosolve Chemie), 1,3-propanesultone 99\% (Alfa Aesar), double polished $\mathrm{Si}_{3} \mathrm{~N}_{4}$ surfaces (LioniX, Enschede, NL), 8-(+)-biotinylamino-3,6-dioxa-octyl (1R,8S,9S)-bicyclo[6.1.0]non-4-yn-9ylmethyl carbamate (BCN-biotin) (SynAffix), Si(100) wafers (n-doped, Addison Engineering, Inc.), Argon 6.0 Instrument (LINDE GAS Benelux B.V.). Deionized water was produced with a MilliQ Integral 3 system (Millipore). For synthesis procedures and characterization of synthesized compounds, see Supporting Information.

\subsubsection{Surface characterization}

\section{SCA}

The wettability of modified surfaces was determined by automated static water contact angle measurements (SCA) using a Krüss DSA100 goniometer. Droplets of MilliQ water ( $3 \mu \mathrm{L})$ were dispensed on the surface and angles were measured with a CCD Camera using a tangential method. The reported values are an average of three equally treated surfaces. For TFAAD- and $\mathrm{NH}_{2}$-modified surfaces, three droplets per surface where measured and averaged, pSB and co(azido-SB) surfaces were measured with 1 droplet per surface.

\section{XPS}

Modified surfaces were analyzed by X-Ray Photoelectron Spectroscopy (XPS) using a JPS-9200 photoelectron spectrometer (JEOL). The spectra were obtained using monochromatic $\mathrm{Al} \mathrm{K \alpha}$ $\mathrm{X}$-Ray radiation at $12 \mathrm{kV}$ and $20 \mathrm{~mA}$, with an analyzer energy pass of $10 \mathrm{eV}$ for narrow scans. The obtained spectra were processed using CASA XPS peak fit program (version 2.3.16 PR 1.6). All high-resolution spectra were corrected with a linear background fitting. 


\section{XRR}

The thickness of obtained layers was determined via X-Ray Reflectivity (XRR) using a X'pert Pro diffraction (PANalytical, Philips) system. The instrumental setup was composed of a goniometer two axes system PW3050/60, a sample stage holder AP TTK-450, an X-Ray generator MPPC (40 kV; $40 \mathrm{~mA}$ ) and a X-Ray tube Empyrean Cu LFF (94300337300x) DK361647. Used incident beam optics had a radius of $240 \mathrm{~mm}$, a take-off angle of $6^{\circ}$ and a fixed incident beam mask of $5 \mathrm{~mm}$. The diffracted beam optics of the used X-Ray source were $\mathrm{K} \alpha(\lambda=1.5418 \AA)$ with a $\mathrm{Cu}$ anode, a soller slit of $0.04 \mathrm{rad}$ and a parallel plate collimator of $0.27^{\circ}$. For each sample, a series of the scattered intensity curves was measured at grazing angles ranging from $0.1^{\circ}$ to $3^{\circ}$.

\section{FT-IR}

Fourier transform infrared (FTIR) spectra were recorded using a Bruker Tensor 27 FTIR spectrometer equipped with a Harrick Autoseagull ${ }^{\mathrm{TM}}$ and an attenuated total reflectance (ATR) germanium crystal using an optimized incident angle of $55^{\circ}$ (1024 scans). As reference a freshly etched double polished stoichiometric $\mathrm{Si}_{3} \mathrm{~N}_{4}$ sample was used. Collected data were processed using the software OPUS 6.5 and are shown without any corrections.

\subsubsection{Surface modification}

\section{$\mathrm{SI}_{3} \mathrm{~N}_{4}$ SUBSTRATE PREPARATION}

A $162 \pm 3 \mathrm{~nm}$ thick layer of stoichiometric silicon nitride $\left(\mathrm{Si}_{3} \mathrm{~N}_{4}\right)$ was deposited by LPCVD on both sides (double polished) of a silicon wafer without intermediate oxide layer, and diced into $1 \times 1 \mathrm{~cm}$ surfaces (Lionix B.V.). The surfaces were rinsed and sonicated for $5 \mathrm{~min}$ in semiconductor grade acetone, rinsed again with the same solvent and dried in a stream of argon. Surfaces were subsequently cleaned from organic material by $5 \mathrm{~min}$ of air-based plasma. The surfaces were etched by immersing them in a $2.5 \%$ aqueous HF solution for 2 min, followed by rinsing with MilliQ water and drying in a stream of argon.

Immediately after etching, the surfaces were placed in a flat-bottomed reaction vessel containing neat TFAAD, which was degassed by three freeze-pump-thaw cycles using liquid nitrogen and argon. After transferring the samples, another two freeze-pump-thaw cycles were performed to remove residual traces of oxygen and/or water. The reaction was carried out at $150{ }^{\circ} \mathrm{C}$ under an argon atmosphere for $16 \mathrm{~h}$. The reaction vessel was allowed to cool down to room temperature for $30 \mathrm{~min}$. To remove any physisorbed TFAAD, the surfaces were rinsed, sonicated for $5 \mathrm{~min}$ and rinsed again with DCM and dried in a stream of argon.

\section{DEPROTECTION}

TFAAD-modified silicon nitride substrates were placed in a $1 \mathrm{M} \mathrm{NaOH}$ solution for 5 min 
under occasional shaking. The reaction was stopped by washing and sonicating the surfaces in MilliQ water for 5 min. The surfaces were then rinsed with MilliQ water, ethanol, and DCM, and dried in a stream of argon.

\section{INITIATOR ATTACHMENT}

Amine-terminated surfaces were placed into a three-necked flat-bottomed flask under inert gas atmosphere. $2.0 \mathrm{~mL}$ of DCM, $0.6 \mathrm{~mL} \alpha$-bromoisobutyryl bromide and $0.5 \mathrm{~mL}$ DIPEA were added via syringes and the flask was placed on a shaker at $80 \mathrm{rpm}$ overnight. Initiatorfunctionalized surfaces were washed with DCM, sonicated in DCM for $5 \mathrm{~min}$, washed another time with DCM and dried under a stream of argon. Surfaces were used directly for surface initiated polymerization.

\section{SURFACE-INITIATED POLYMERIZATION}

Surface-initiated Atom Transfer Radical Polymerization (ATRP) was performed under inert gas atmosphere. All solvents were degassed prior to use by $5 \mathrm{~min}$ sonication and $30 \mathrm{~min}$ argon bubbling. $16.1 \mathrm{mg}\left(0.16 \mathrm{mmol}, 0.45\right.$ eq.) of an $\mathrm{Cu}(\mathrm{I}) \mathrm{Cl} / \mathrm{Cu}(\mathrm{II}) \mathrm{Cl}_{2}(9 / 1)$ mixture and $54.7 \mathrm{mg}$ (0.35 mmol, 1.0 eq.) Bpy were dissolved in $7 \mathrm{~mL}$ of an isopropanol/MilliQ water $(20 / 80)$ mixture and stirred for $30 \mathrm{~min} .3 .3 \mathrm{~mL}$ of the resulting brown complex solution was transferred to a flask containing the respective monomer mixture and stirred until full solubilization (pSB: $876 \mathrm{mg} \mathrm{SB}$; co-5 $5 \%$ : $833 \mathrm{mg} \mathrm{SB}$ and $54.2 \mathrm{mg} \mathrm{5}$; co-5 ${ }_{15 \%}: 746 \mathrm{mg} \mathrm{SB}$ and $163 \mathrm{mg} \mathrm{5}$ ). (The required amount of monomer $\mathbf{5}$ was taken from a thawed aliquot (see synthesis of $\mathbf{5}$ ) and placed into a Schlenk flask and the water removed under vacuum, before the standard monomer was added.) An initiator-modified surface was placed separately and diagonally into a Schlenk tube with the modified side downwards, enabling stirring underneath the surface. $1.1 \mathrm{~mL}$ of the prepared ATRP solution was transferred into it. After stirring for $1 \mathrm{~h}$ at RT, the polymer brush-modified surface was washed with $20 \mathrm{~mL}$ of $60{ }^{\circ} \mathrm{C}$ MilliQ water, sonicated for 5 min in MilliQ water, washed with $10 \mathrm{~mL}$ of MilliQ water and acetone, respectively, and dried in a stream of argon.

\subsubsection{Brush functionalization}

\section{AZIDO TOP EXCHANGE}

Exchanging the terminal bromide of pSB for an azide was done by placing the surfaces in an aqueous solution of $\mathrm{NaN}_{3}(50 \mathrm{mM})$ and $\mathrm{NaI}(50 \mathrm{mM})$. After shaking at $80 \mathrm{rpm}$ for $6 \mathrm{~h}$, the surfaces were rinsed with MilliQ and dried in an argon stream.

\section{SPAAC}

The obtained surfaces were wetted with $20 \mu \mathrm{L}$ (the minimum amount used to wet a $1 \times 1 \mathrm{~cm}$ surface for most efficient use of resources) of a $20 \mathrm{mM}$ solution of $\mathrm{BCN}_{-} \mathrm{CF}_{3}$ or $\mathrm{BCN}$-biotin in 
DMSO, respectively, and stored in a Petri dish overnight. The surfaces were placed for $1 \mathrm{~h}$ in pure DMSO and another $1 \mathrm{~h}$ in ethanol to remove non reacted material, washed with ethanol and dried in an argon stream.

\subsubsection{Reflectometry}

Antifouling and selective binding studies were performed using a self-made reflectometer, with a similar set-up as previously described. ${ }^{33-34}$ A monochromatic linearly polarized laser $(632.8 \mathrm{~nm})$ passes a glass prism, which results in an angle of incidence of $68^{\circ}$ (the optimal angle was determined using Prof. Huygens software) with the solvent-substrate interface. The reflected light is refracted by the glass prism and split into its p- and s-polarized components (relative to the plane of incidence) by a beam splitter. The ratio between the separately measured intensities of the $\mathrm{p}$ - and s-polarized components results in the output signal S. The set-up is combined with a stagnation point flow cell, enabling protein adsorption measurements under diffusion-controlled conditions.

\section{SPECIFIC AND NON-SPECIFIC PROTEIN BINDING.}

A stock solution of $10 \times$ PBS (ionic strength $0.8 \mathrm{M}$ ) was prepared (phosphate buffered saline, pH 6.7, composition: $37.0 \mathrm{~g} \mathrm{~L}^{-1} \mathrm{NaCl}, 1.0 \mathrm{~g} \mathrm{~L}^{-1} \mathrm{KCl}, 2.0 \mathrm{~g} \mathrm{~L}^{-1} \mathrm{KH}_{2} \mathrm{PO}_{4}$ and $2.0 \mathrm{~g} \mathrm{~L}^{-1} \mathrm{Na}_{2} \mathrm{HPO}_{4}$ ). Protein solutions were prepared by dissolving fibrinogen $\left(0.5 \mathrm{mg} \mathrm{mL}^{-1}\right)$, avidin $\left(0.1 \mathrm{mg} \mathrm{mL}^{-}\right.$ $\left.{ }^{1}\right)$ and a mixture of both $\left(0.5 \mathrm{mg} \mathrm{mL}^{-1}\right.$ of fibrinogen and $0.1 \mathrm{mg} \mathrm{mL}^{-1}$ avidin $)$ in $2 \times \mathrm{PBS}$ (ionic strength $0.16 \mathrm{M}$ ) and placed on a shaker at $37^{\circ} \mathrm{C}$ at $80 \mathrm{rpm}$ for $30 \mathrm{~min}$ to ensure full solubilization. ${ }^{\mathrm{S} 4}$ Prior to use, the solutions were two times diluted with MilliQ water to a final ionic strength of $0.08 \mathrm{M}$. Surfaces of roughly $1 \times 4 \mathrm{~cm}$ were required for the reflectometry measurements. In order to measure the pre-cut $1 \times 1 \mathrm{~cm} \mathrm{Si}_{3} \mathrm{~N}_{4}$ surfaces, $1 \times 4 \mathrm{~cm}$ strips were cut from a $\mathrm{Si}(100)$ wafer and the surface of interest glued onto one end of the silicon strip, while a dummy $\mathrm{Si}_{3} \mathrm{~N}_{4}$ surface was glued on the other side for clamping in the reflectometer. A two components epoxy glue (KOMBI SNEL ${ }^{\circledR}$, Bison) was used. The glued surfaces were allowed to dry overnight. The surfaces were placed $1 \mathrm{~h}$ before the measurement into $1 \times$ PBS to avoid artefacts caused by swelling of the polymer brushes during measurements.

All reflectometry measurements were carried out at room temperature. Each reflectometry measurement consisted of a $400 \mathrm{sec}$ injection with PBS, followed by an adsorption/binding phase of $1000 \mathrm{sec}$ and a desorption/washing phase of at least $400 \mathrm{sec}$. The amount of protein adsorbed or bound to each surface was determined in the plateau of the washing phase of the obtained curve. All experiments were carried out in triplicate. Data processing was done using Origin (version 8) software, binding curves were smoothened using the 50\% percentile filtering option. In order to get the FIB adsorption on bare $\mathrm{Si}_{3} \mathrm{~N}_{4}$ and brush-coated surfaces on the same scale, noise levels (before smoothing) were compared and adjusted accordingly. 


\subsection{Results and discussion}

\subsubsection{Synthesis and characterization of zwitterionic monomers}

The standard sulfobetaine (SB) monomer was obtained via the well-established one-step nucleophilic ring opening reaction of sultones ${ }^{3-5}$ (for more details see Supporting Information Scheme S2.8). However, this approach failed in the presence of an N-azide substituted starting material due to the reduced nucleophilicity of the tertiary nitrogen. The novel clickable and<smiles>C=C(C)C(=O)NCCCN(C)C</smiles>
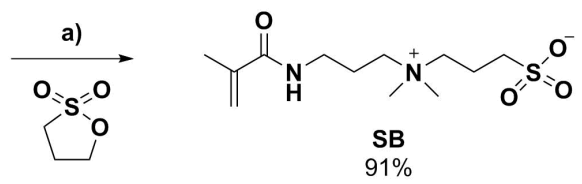<smiles>CN(CCCN)CCCN</smiles>

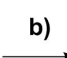<smiles>CN(CCCN)CCCNC(=O)OC(C)(C)C</smiles><smiles>CC(C)(C)OC(=O)NCCC[N+](C)(CCCN)CCCS(=O)(=O)[O-]</smiles><smiles>O=S(=O)(CCCBr)O[Na]</smiles>

c)<smiles>NS(=O)(=O)c1ccn(S(=O)(=O)O[Na])c1</smiles>
e)<smiles>CNCCC[N+](C)(CCCN)CCCS(=O)(=O)[O-]</smiles><smiles>CC=C(C)C(=O)O</smiles><smiles>CN(CCC[18OH])CCCNC(=O)OC(C)(C)C</smiles><smiles>C=C(C)C(=O)NCCC[N+](C)(CCCN)CCCS(=O)(=O)[O-]</smiles>

Scheme 2.1 top) Synthesis of standard monomer SB: a) acetone, RT, overnight, 91\%; (bottom) Synthesis of clickable monomer 5: b) $\mathrm{Boc}_{2} \mathrm{O}, \mathrm{DCM}, 0{ }^{\circ} \mathrm{C}$ to RT, overnight, $82 \%$; c) DTR, $\mathrm{CuSO} 4, \mathrm{~K} 2 \mathrm{CO} 3, \mathrm{MeOH}$, RT, overnight, 59\%; d) 3 bromopropane sulfonic acid sodium salt, DMF, $70{ }^{\circ} \mathrm{C}, 4 \mathrm{~d}, 73 \%$; e) $4 \mathrm{M} \mathrm{HCl}$, dioxane, 24 h, RT, quant.; f) methacrylic acid, HOBt, EDCI·HCl, NMM, DMF s.g., 2 d, RT, 80\%. 
zwitterionic monomer $\mathbf{5}$ was synthesized in an alternative route in five steps as shown in Scheme 2.1. Standard mono-protection of an appropriate diamine followed by conversion of the free amine into the azide succeeded in moderate to good yields. The zwitterionic character was introduced in a nucleophilic substitution reaction of the tertiary amine. Finally, deprotection and introduction of the polymerizable methacrylamide unit via standard peptide coupling in the last step, resulted in the desired monomer 5 in good overall yield (28\%, typically yielding $5 \mathrm{~g}$ of final product per route; see Supporting Information) and high purity. Since $1 \mathrm{~g}$ of $\mathbf{5}$ is enough for the formation of more than 250 substrates of $1 \times 1 \mathrm{~cm}$ coated with co-5 $\mathbf{5}_{1 \%}$ polymer brushes (see below for details), this synthesis is sufficiently efficient for large-scale preparation of 3D-functionalizable fully-zwitterionic polymer brushes.

\subsubsection{Surface modification and grafting of functional copolymers}

In contrast to silicon-enriched silicon nitride, ${ }^{36}$ the modification of so-called stoichiometric $\mathrm{Si}_{3} \mathrm{~N}_{4}$, which is commonly used in optofluidic sensing devices, requires harsher conditions under which the ATRP initiator (tertiary bromide) is not stable. Consequently we developed an alternative route, in which first an amine-terminated monolayer is introduced (Scheme 2.1ac). To this end, we used a heat-induced alkene-based covalent attachment of trifluoroacetamide decene (TFAAD) on cleaned $\mathrm{Si}_{3} \mathrm{~N}_{4}$ substrates (for an efficient scaled-up synthesis of TFAAD, see Supporting Information), analogous to what was recently reported for GaN surfaces. ${ }^{37}$ This was followed by deprotection of the base-labile trifluoroacetamide group to yield the amineterminated monolayer. Successful introduction and subsequent removal of fluorine during these steps was shown by XPS analysis and contact angle measurements. The characteristic surface composition $^{38}$ of the TFAAD monolayer is visible from the XPS carbon narrow scan, clearly showing four peaks (Figure 2.2, for more detailed XPS analysis see Supporting Information Figure S2.2-Figure S2.4).

The absence of the $\mathrm{CF}_{3}$ and the carbonyl signal after basic treatment, confirmed the successful removal of the trifluoroacetamide group. The static water contact angle decreased from $71 \pm 1^{\circ}$ $\left(\mathrm{CF}_{3}\right.$-terminated monolayer) to $55 \pm 1^{\circ}$ due to the more hydrophilic primary amine groups, which is in good agreement with previously reported values by Carvalho et al. ${ }^{39}$ This fast and efficient route towards amine-terminated monolayers is a useful tool and widely applicable to other substrates, including glass. ${ }^{39}$

The acid bromide-based ATRP initiator was introduced according to previously described procedures (Scheme 2.2c), yielding an increase in static water contact angles to $73 \pm 1^{\circ}$, in accordance with reported values $\left(72^{\circ}\right.$ ) (see Supporting Information Figure S2.5 for XPS spectra). ${ }^{23}$ Subsequently, surface-initiated polymerization was performed, using optimized conditions of previously reported ATRP procedures (including scale, time and stirring conditions) to ensure the formation of homogeneous polymer brush coatings. ${ }^{9,22,34}$ The fraction of azide functionalities in the resulting polymer brush could be controlled straightforwardly by copolymerization of variable amounts of $\mathbf{5}$ with respect to the standard SB monomer, whilst 
fully maintaining the zwitterionic character (Scheme 2.2, route '3D'). Polymer brushes with $0 \%(\mathrm{pSB}), 5-\%\left(\mathrm{CO}-5_{5 \%}\right)$ and $15 \%\left(\mathrm{CO}-5_{15 \%}\right)$ of $\mathbf{5}$ were grown and all showed characteristic static water contact angles of below $20^{\circ}$ (for pictures of SCA measurements see Supporting Information Table S2.1). This indicates a preserved highly hydrophilic character of the copolymer brushes. The increasing azide content could be visualized by G-ATR-FTIR measurements (Figure 2.5A), while the thickness of the obtained polymer coatings was measured by XRay Reflectivity and appeared to be $51 \pm 2 \mathrm{~nm}$ (see Supporting Information Table S2.2).

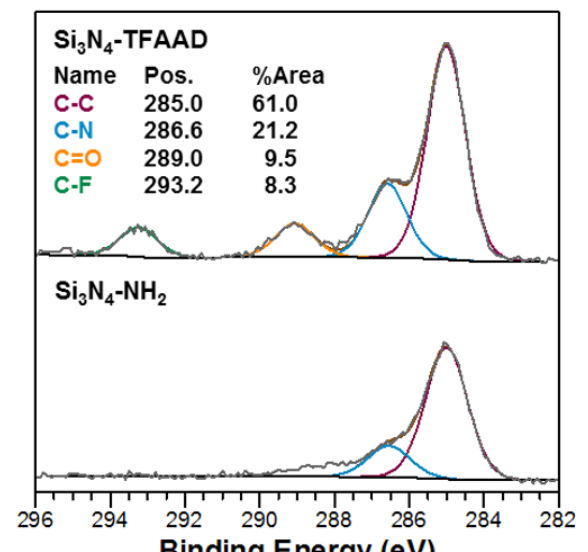

Binding Energy (eV)

Figure 2.2 XPS C1s narrow scan spectra of (top) TFAAD-modified and (bottom) deprotected, amineterminated $\mathrm{Si}_{3} \mathrm{~N}_{4}$ substrates.

XPS analysis clearly confirmed the presence of azide moieties within the copolymer brush (Figure 2.3). Beside the characteristic $1: 1$ ratio of the amide (397.8 eV) and ammonium signal $(400.6 \mathrm{eV})$, two new nitrogen signals in the N $1 \mathrm{~s}$ spectra of the co-5 ${ }_{5 \%}$ and co-5 $\mathbf{1 5}_{15}$-brush at $398.7 \mathrm{eV}$ and $402.6 \mathrm{eV}$ (in a ratio of $1: 2$ ) could be discerned, which can be attributed to the three nitrogen atoms of the introduced azide. The obtained ratios of the intensity of the azide with respect to the ammonium signal give rise to the actual copolymer composition, which was calculated to be $5 \%$ for the co- $\mathbf{5}_{5 \%}$ and $\mathbf{1 3 \%}$ for the co- $\mathbf{5}_{15 \%}$ brushes, i.e. in agreement with the monomer feed ratio.

For reference, we also prepared top-functionalized brushes using the previously reported 2D approach by our group (Scheme 2.2, route '2D'). ${ }^{22}$ To this end, ATRP with the standard SB monomer was performed, followed by top exchange of the terminal halogen in a nucleophilic substitution reaction using sodium azide in an extra step. Because a maximum of only one azide moiety per polymer chain was introduced in these $\mathrm{pSB}-\mathrm{N}_{3}$ brushes, no azide signal could be observed by XPS (for XPS spectra see Supporting Information Figure S2.8) or G-ATR-FTIR (Figure 2.5A), which is in stark contrast with the straightforward detection of azides in the copolymers discussed above. 

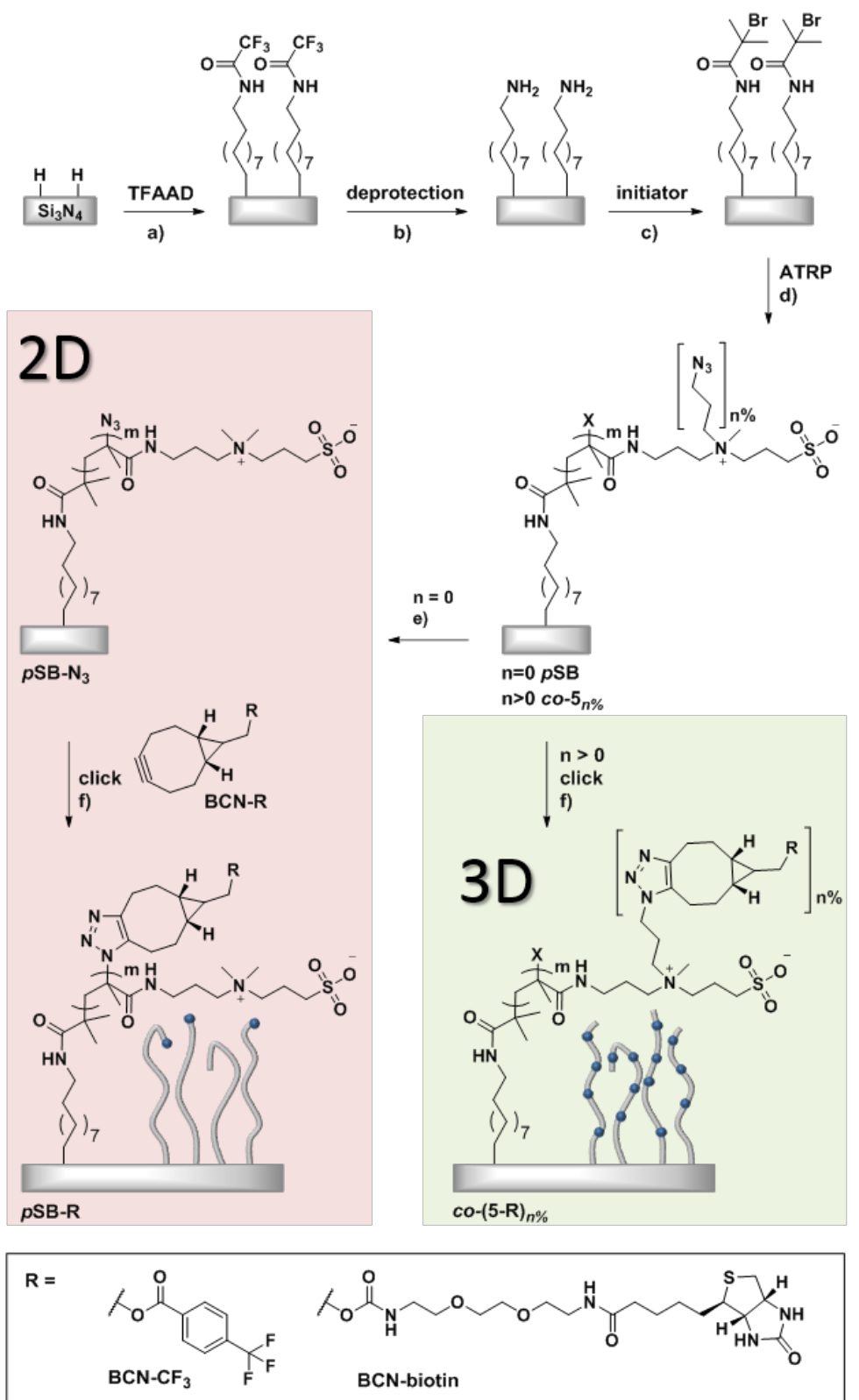

Scheme 2.2 Stepwise modification of $\mathrm{Si}_{3} \mathrm{~N}_{4}$ surfaces yielding functionalized copolymer brushes: a) TFAAD neat, $150{ }^{\circ} \mathrm{C}, 16 \mathrm{~h}$; b) $1.0 \mathrm{M} \mathrm{NaOH}, \mathrm{RT}, 5 \mathrm{~min}$; c) $\alpha$-bromoisobutyryl bromide, DCM, RT, $24 \mathrm{~h}$; d) $100-\mathrm{n} \% \mathrm{SB}, \mathrm{n} \%$ 5, Cu(I)/Cu(II) (90/10), Bpy, isopropanol/water (20/80), RT, 2 h; e) $50 \mathrm{mM}$ aq. $\mathrm{NaN}_{3}$, $50 \mathrm{mM}$ NaI, 6 h, RT; f) BCN-R, DMSO, RT, 16 h. 


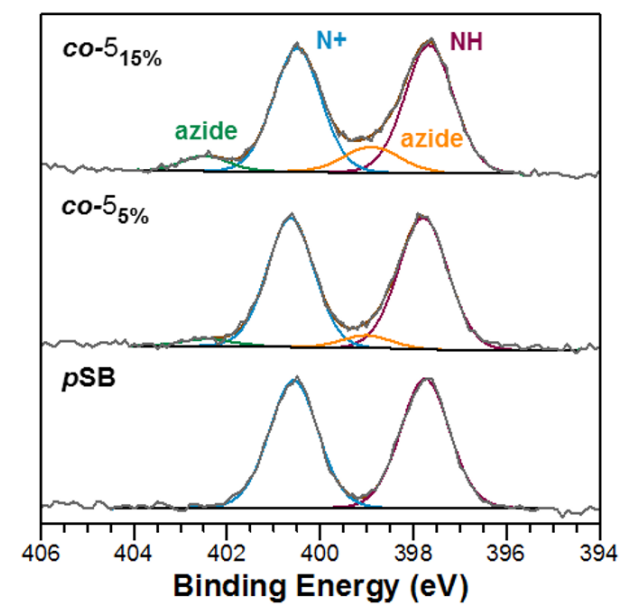

Figure 2.3 XPS nitrogen narrow scan spectra of azido-copolymers with various content of clickable monomer 5: pSB, CO-5 $5_{5 \%}$ and co-5 ${ }_{15 \%}$ (for narrow scans of C 1s region see Supporting Information Figure S2.6-S2.8).

To demonstrate the effective functionalization of the azide moieties in the copolymer brush, these groups were first of all reacted with a $\mathrm{CF}_{3}$-labeled bicyclooctyne $\left(\mathrm{BCN}_{-} \mathrm{CF}_{3}\right)$ (under SPAAC conditions, see Scheme 2.2). Due to their relatively high sensitivity factor, fluorine atoms can be sensitively measured by XPS. As a result, small amounts of as well as minute changes in fluorine content can be detected, which thus allows for a detailed characterization of the functionalized brushes. XPS wide scan spectra of the resulting BCN-CF functionalized $\mathrm{CO}-\left(5-\mathrm{CF}_{3}\right)_{5 \%}{ }^{-}$and co- $\left(5-\mathrm{CF}_{3}\right)_{15 \%}$-polymer brushes showed a clear increase in the fluorine signal intensity upon raising the content of 5 from $0 \%$ via $5 \%$ (Figure 2.4A) to $15 \%$ (Figure 2.4B). Based on the atomic ratios, $85 \pm 1 \%$ of the azide moieties had been successfully converted for both the CO- $5_{5 \%}$ - and $\mathrm{CO}-\mathbf{5}_{15 \%}$-coatings, which indicates that the SPAAC reaction had occurred clearly beyond the solution interface. XPS nitrogen narrow scan spectra (Figure 2.4C) of co- $\left(5-\mathrm{CF}_{3}\right)_{\mathrm{n} \%}-$ polymer brushes showed the disappearance of the characteristic azide signal at $402.6 \mathrm{eV}$ under formation of a new signal at $399.5 \mathrm{eV}$. The latter signal can be attributed to the nitrogen atoms from the triazole ring that is formed during the SPAAC reaction. The incorporation of fluorine is furthermore visible in the carbon narrow scan as a fluorine-bound carbon signal, showing a characteristic shift to higher binding energies of $292.9 \mathrm{eV}$ (Figure 2.4D). The functionalization of chain end-modified $\mathrm{pSBN}_{3}$-brushes could, in line with the inability to detect the azide signal, not be detected by XPS (for spectra see Supporting Information Figure S2.9).

As XPS only allows analysis of the top $\sim 20 \mathrm{~nm}$ of the brush, complementing G-ATR-FTIR measurements were performed to provide insight into the full layer composition of the grafted copolymer brushes. The increasing amount of azide within the grafted copolymer chain was visualized by an increasing intensity of the characteristic azide stretching vibration at $2104 \mathrm{~cm}^{-1}$ (Figure 2.5A). 

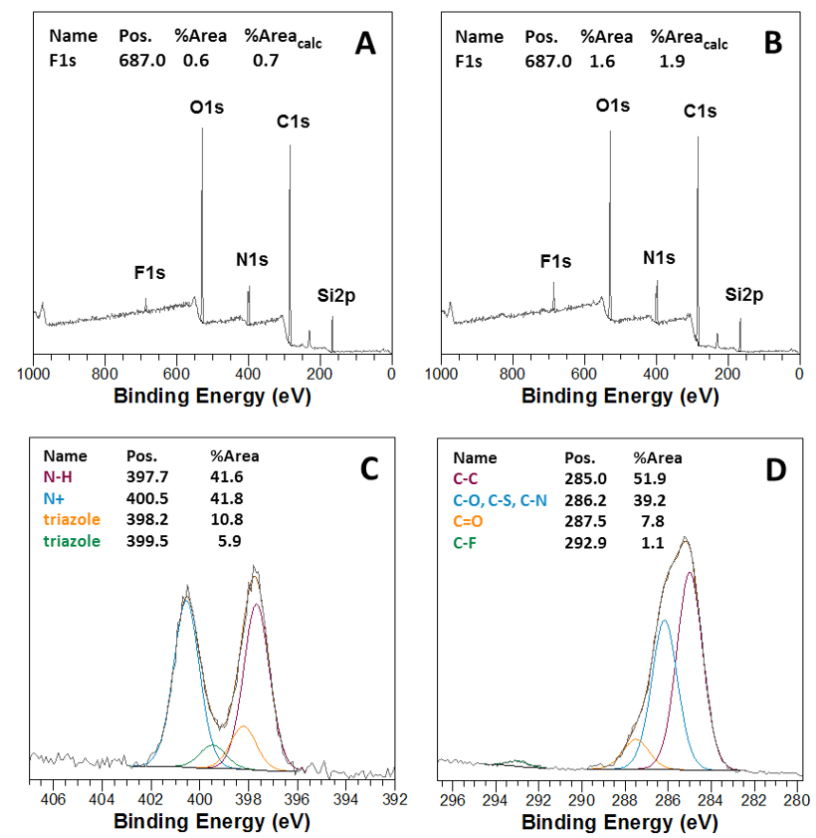

Figure 2.4 (top) XPS wide scan spectra after SPAAC reaction of azido-copolymer coatings on $\mathrm{Si}_{3} \mathrm{~N}_{4}$ with $\mathrm{BCN}-\mathrm{CF}_{3}$ : A) co-(5-CF3) $)_{5 \%}$ - and B) co- $\left(5-\mathrm{CF}_{3}\right)_{15 \%}$-polymer brush; (bottom) exemplary N 1s (C) and C 1s (D) narrow scan of co- $\left(5-\mathrm{CF}_{3}\right)_{15 \%}$-polymer brush (for narrow scan spectra of co- $\left(5-\mathrm{CF}_{3}\right)_{5 \%}$-polymer brush see Supporting Information Figure S2.10).

The spectra also display the characteristic carbonyl stretching at $1646 \mathrm{~cm}^{-1}$ and $\mathrm{N}-\mathrm{H}$ bending vibration at $1534 \mathrm{~cm}^{-1}$ of amides as well as the $\mathrm{C}-\mathrm{N}$ stretching vibration at $1485 \mathrm{~cm}^{-1}$. Figure $2.5 \mathrm{~B}$ shows the spectra before and after fluorine labeling of $\mathrm{CO}^{-5}{ }_{15 \%}$-polymer brush with $\mathrm{BCN}$ $\mathrm{CF}_{3}$. The characteristic azide stretching vibration at $2104 \mathrm{~cm}^{-1}$ disappeared due to conversion into a triazole heterocycle after SPAAC reaction. In addition, the carbonyl stretching vibration of the $\mathrm{BCN}-\mathrm{CF}_{3}$ ester appears at $1718 \mathrm{~cm}^{-1}$. These combined XPS and G-ATR-FTIR findings confirm the successful and uniform 3D-functionalization of the brush coating throughout its entire thickness.

\subsubsection{Sensing performance of co-(5-biotin) ${ }_{n \%}$ coatings}

The sensing capability of the presented azido-copolymer brush coatings as bioactive substrates was investigated by measuring their antifouling and specific binding capacities with reflectometry, following an earlier reported procedure by our group. ${ }^{34}$ The antifouling properties were evaluated by measuring the non-specific adsorption of fibrinogen (FIB), a large protein from blood plasma that strongly binds to hydrophobic surfaces. ${ }^{40}$ The relatively 
high concentration $\left(0.5 \mathrm{mg} \mathrm{mL}^{-1}\right)$ used in this study ensures not only fast equilibration, but more importantly also presents challenging fouling conditions. To assess the specific protein binding, the well-studied biotin-avidin interaction was chosen. ${ }^{41-42} \mathrm{Co}-\mathbf{5}_{\mathrm{n} \%}$-polymer brushcoated surfaces were compared to 2D top-functionalized $\mathrm{pSBN}_{3}$ polymer brushes. All substrates were reacted with BCN-biotin using the aforementioned SPAAC reaction (see Scheme 2.2). In this way, top-functionalized pSB-biotin polymer brushes as well as co-(5-biotin $)_{1 \%}$ and co-(5biotin) ${ }_{5 \%}$ copolymer coatings were prepared. Figure 2.6 summarizes the obtained reflectometry data. While on bare $\mathrm{Si}_{3} \mathrm{~N}_{4}$ substrates strong fouling by FIB was observed, all brush coatings fully preserved their antifouling properties, independently of the amount of introduced biotin recognition units (FIB binding $\sim 0$ RU, Figure 2.6A). From the experiments with avidin (Figure $2.6 \mathrm{~B}$ ), it can firstly be concluded that the specific binding of avidin to chain end-modified pSBbiotin did not result in a significant signal and could not be distinguished from a standard pSB brush without biotin units. This means that under these conditions no specific binding could be discerned for the top-functionalized brush, which can be attributed to the challengingly thick polymer brush coatings used in this study (exceeding $35 \mathrm{~nm}$; for respective thicknesses see Supporting Information Table S2.2). While such thicknesses are optimal for antifouling purposes, for such brushes the chain ends are more buried and therefore less accessible for modification as compared to those on relatively thin polymer layers. ${ }^{34}$ Secondly, and in sharp contrast, our homogeneously 3D-modified co-(5-biotin) $)_{\mathrm{n} \%}$ polymer brush coatings both yielded a clear avidin response. Co-(5-biotin) ${ }_{1 \%}$ surfaces showed a binding of $0.28 \pm 0.04 \mathrm{RU}$; this value increased by a factor of 2.6 for co-(5-biotin) $)_{5 \%}$ surfaces $(0.72 \pm 0.02 \mathrm{RU})$. The non-

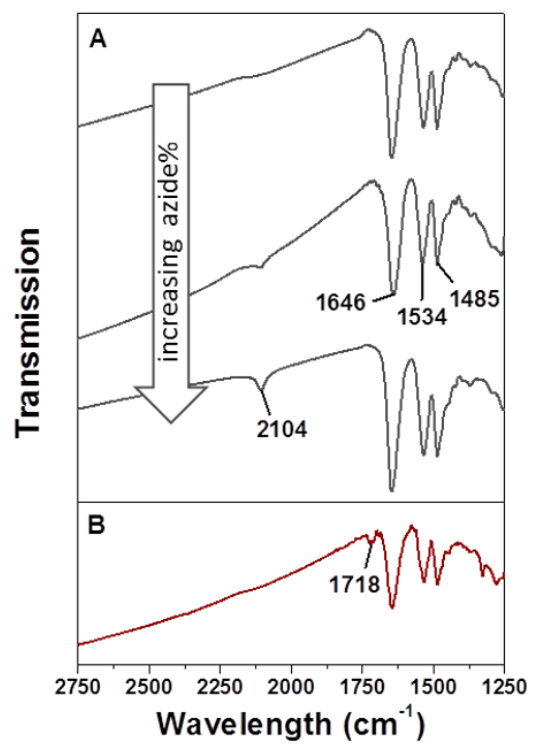

Figure 2.5 (Comparison of G-ATR-FTIR spectra of A) pSB, co-5 ${ }_{5 \%}$, co- $\mathbf{5}_{15 \%}$-polymer brushes grafted from $\mathrm{Si}_{3} \mathrm{~N}_{4}$-initiator substrates and B) co- $\left(\mathbf{5}-\mathrm{CF}_{3}\right)_{15 \%}$-polymer brush after SPAAC reaction of co- $\mathbf{5}_{15 \%}{ }^{-}$ polymer brush with $\mathrm{BCN}-\mathrm{CF}^{3}$. 
linear increase from 1 to $5 \%$ biotin modification can be attributed to a surface coverage with avidin that approaches full coverage in case of the co-(5-biotin) ${ }_{5 \%}$ brushes, leaving some of the biotin units unbound.

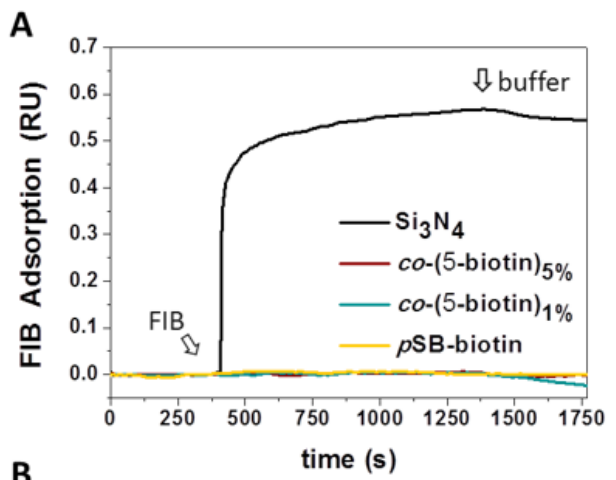

B
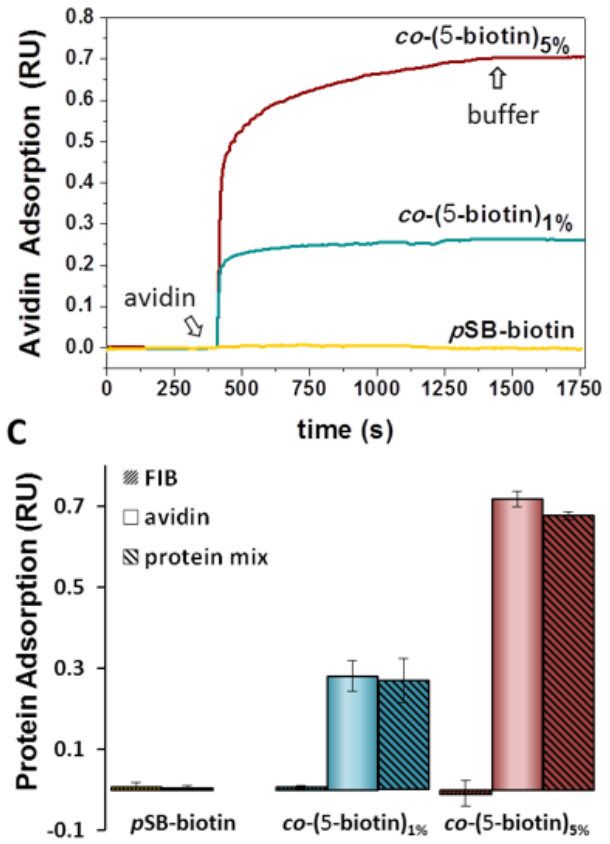

Figure 2.6 Protein adsorption and binding onto bare and polymer brush-coated $\mathrm{Si}_{3} \mathrm{~N}_{4}$ surfaces as measured by reflectometry. (A) FIB $\left(0.5 \mathrm{mg} \mathrm{mL}{ }^{-1}\right)$ adsorption onto bare $\mathrm{Si}_{3} \mathrm{~N}_{4}, \mathrm{pSB}$, co- $\mathbf{5}_{1 \%}$ and co- $\mathbf{5}_{5 \%}{ }^{-}$ polymer brushes; (B) binding curves of avidin $\left(0.1 \mathrm{mg} \mathrm{mL}^{-1}\right)$ on BCN-biotin-functionalized pSB- $\mathrm{N}_{3}$, $\mathrm{CO}^{-\mathbf{5}_{1 \%}}$ and $\mathrm{CO}-\mathbf{5}_{5 \%}$-polymer brushes. (C) Bar plot summarizing FIB adsorption $\left(0.5 \mathrm{mg} \mathrm{mL}^{-1}\right)$ and avidin binding of pure avidin $\left(0.1 \mathrm{mg} \mathrm{mL}^{-1}\right)$ and a protein mix solution of $0.1 \mathrm{mg} \mathrm{mL}^{-1}$ avidin and $0.5 \mathrm{mg} \mathrm{mL}^{-1}$ FIB onto the various functionalized surfaces. 
For sensing purposes, it is required to be able to specifically bind a distinct protein or other analyte from a protein mixture without the interference of other (bio)molecules present, thus functioning as a romantic surface. ${ }^{43}$ To this end, the specific binding of avidin was measured from a $1: 5$ protein mixture of avidin $\left(0.1 \mathrm{mg} \mathrm{mL}^{-1}\right)$ and FIB $\left(0.5 \mathrm{mg} \mathrm{mL}^{-1}\right)$. As depicted in Figure $2.6 \mathrm{C}$, the avidin binding from the protein mix was (within experimental error) identical to the binding of avidin from a single protein solution, demonstrating that the binding of the target protein is not hampered by aspecific protein binding. With this we show that the use of azide-modified copolymer brush coatings as presented in this study, is a straightforward, generally applicable approach to create high performance (bio)active sensing platforms for the effective and specific binding of an analyte, while fully preserving optimal antifouling conditions.

\subsection{Conclusion}

In conclusion, the concept of unifying zwitterionic character and clickable moieties in a single monomer, as demonstrated here for monomer 5, enables formation of 3D-functionalizable antifouling brushes by copolymerization of this monomer with a standard zwitterionic SB monomer. The introduced azide moieties are homogeneously distributed throughout the polymer brush, leading to an efficient and thickness-independent brush modification. The introduced amount of azide groups can be adjusted to the required application by control over the monomer ratio (e.g. depending on size of the analyte to be bound). Subsequent modification with a recognition unit of interest, yields a highly efficient sensing platform for selective binding with retained, excellent antifouling properties. Incorporation of $1 \%-5 \%$ of monomer $\mathbf{5}$ was shown to achieve an unprecedented degree of loading in a proof of principle sensing assay using the well-known biotin-avidin interaction. We anticipate this new approach to be of general interest for sensing applications that demand excellent antifouling performance in combination with a tunable degree of functionalization.

\subsection{Acknowledgements}

The authors gratefully acknowledge Remco Fokkink and Barend van Lagen for assisting with reflectometry and XRR measurements, respectively. This work was supported by the BIOFOS specific-target research program (STREP) co-funded by the European Commission under the 7th Framework Programme and NanoNextNL (program 3E), a micro- and nanotechnology consortium of the Government of The Netherlands and 130 partners.

\subsection{References}

1. Blaszykowski, C.; Sheikh, S.; Thompson, M. A Survey of State-of-the-Art Surface Chemistries to Minimize Fouling from Human and Animal Biofluids. Biomater. Sci. 2015, 3, 1335-1370.

2. Huang, C. J.; Li, Y.; Krause, J. B.; Brault, N. D.; Jiang, S. Internal Architecture of Zwitterionic Polymer Brushes Regulates 
Nonfouling Properties. Macromol. Rapid Commun. 2012, 33, 1003-1007.

3. Zhang, Z.; Chao, T.; Chen, S.; Jiang, S. Superlow Fouling Sulfobetaine and Carboxybetaine Polymers on Glass Slides. Langmuir 2006, 22, 10072-10077.

4. Cho, W. K.; Kong, B.; Choi, I. S. Highly Efficient Non-Biofouling Coating of Zwitterionic Polymers: Poly((3(Methacryloylamino)Propyl)-Dimethyl(3-Sulfopropyl)Ammonium Hydroxide). Langmuir 2007, 23, 5678-5682.

5. Quintana, R.; Janczewski, D.; Vasantha, V. A.; Jana, S.; Lee, S. S. C.; Parra-Velandia, F. J.; Guo, S.; Parthiban, A.; Teo, S. L.-M.; Vancso, G. J. Sulfobetaine-Based Polymer Brushes in Marine Environment: Is There an Effect of the Polymerizable Group on the Antifouling Performance? Colloids Surf. B Biointerfaces 2014, 120, 118-124.

6. Schlenoff, J. B. Zwitteration: Coating Surfaces with Zwitterionic Functionality to Reduce Nonspecific Adsorption. Langmuir 2014, 30, 9625-9636.

7. Shao, Q.; Jiang, S. Molecular Understanding and Design of Zwitterionic Materials. Adv. Mater. 2015, 27, 15-26.

8. Blaszykowski, C.; Sheikh, S.;Thompson, M. Surface Chemistry to Minimize Fouling from Blood-Based Fluids. Chem. Soc. Rev. 2012, 41, 5599-5612.

9. Krishnamoorthy, M.; Hakobyan, S.; Ramstedt, M.; Gautrot, J. E. Surface-Initiated Polymer Brushes in the Biomedical Field: Applications in Membrane Science, Biosensing, Cell Culture, Regenerative Medicine and Antibacterial Coatings. Chem. Rev. 2014, 114, 10976-11026.

10. Imato, K.; Ohishi, T.; Nishihara, M.; Takahara, A.; Otsuka, H. Network Reorganization of Dynamic Covalent Polymer Gels with Exchangeable Diarylbibenzofuranone at Ambient Temperature. J.Am. Chem. Soc. 2014, 136, 11839-11845.

11. Pyun, J.; Kowalewski, T.; Matyjaszewski, K. Synthesis of Polymer Brushes Using Atom Transfer Radical Polymerization. Macromol. Rapid Commun. 2003, 24, 1043-1059.

12. Zhao, B.; Brittain, W. J. Polymer Brushes: Surface-Immobilized Macromolecules. Prog. Polym. Sci. 2000, 25, 677-710.

13. Matyjaszewski, K.; Tsarevsky, N. V. Macromolecular Engineering by Atom Transfer Radical Polymerization. J. Am. Chem. Soc. 2014, 136, 6513-6533.

14. Matyjaszewski, K. Atom Transfer Radical Polymerization (ATRP): Current Status and Future Perspectives. Macromolecules 2012, 45, 4015-4039.

15. Jiang, H.; Xu, F.-J. Biomolecule-Functionalized Polymer Brushes. Chem. Soc. Rev. 2013, 42, 3394-3426.

16. Zhang, Z.; Chen, S.; Jiang, S. Dual-Functional Biomimetic Materials: Nonfouling Poly(Carboxybetaine) with Active Functional Groups for Protein Immobilization. Biomacromolecules 2006, 7, 3311-3315.

17. Huang, C. J.; Li, Y.; Jiang, S. Zwitterionic Polymer-Based Platform with Two-Layer Architecture for Ultra Low Fouling and High Protein Loading. Anal. Chem. 2012, 84, 3440-3445.

18. Wu, L.; Jasinski, J.; Krishnan, S. Carboxybetaine, Sulfobetaine, and Cationic Block Co-polymer Coatings: A Comparison of the Surface Properties and Antibiofouling Behavior. J.Appl. Polym. Sci. 2012, 124, 2154-2170.

19. Li, B.;Yu, B.;Ye, Q.; Zhou, F. Tapping the Potential of Polymer Brushes through Synthesis. Acc. Chem. Res. 2015, 48, $229-237$.

20. Kitano, H.; Suzuki, H.; Matsuura, K.; Ohno, K. Molecular Recognition at the Exterior Surface of a Zwitterionic Telomer Brush. Langmuir 2010, 26, 6767-6774.

21. Song, L.; Zhao, J.; Luan, S.; Ma, J.; Liu, J.; Xu, X.; Yin, J. Fabrication of a Detection Platform with Boronic-Acid-Containing Zwitterionic Polymer Brush. ACS Appl. Mater. Interfaces 2013, 5, 13207-13215.

22. Nguyen, A. T.; Baggerman, J.; Paulusse, J. M.; Zuilhof, H.; van Rijn, C. J. Bioconjugation of Protein-Repellent Zwitterionic Polymer Brushes Grafted from Silicon Nitride. Langmuir 2012, 28, 604-610.

23. Li, Y.; Giesbers, M.; Gerth, M.; Zuilhof, H. Generic Top-Functionalization of Patterned Antifouling Zwitterionic Polymers on Indium Tin Oxide. Langmuir 2012, 28, 12509-12517.

24. Spiliopoulos, N.; Koutsioubas, A. G.; Anastassopoulos, D. L.; Vradis, A. A.; Toprakcioglu, C.; Menelle, A.; Mountrichas, G.; Pispas, S. Neutron Reflectivity Study of Free-End Distribution in Polymer Brushes. Macromolecules 2009, 42, 6209-6214.

25. Passaro, V. M. N.; Troia, B.; Notte, M. L.; Leonardis, F. D. Photonic Resonant Microcavities for Chemical and Biochemical 
Sensing. RSC Adv. 2013, 3, 25-44.

26. Heideman, R.; Hoekman, M.; Schreuder, E. Triplex-Based Integrated Optical Ring Resonators for Lab-on-a-Chip and Environmental Detection. IEEE J. Sel.Top. Quantum Electron. 2012, 18, 1583-1596.

27. Carlborg, C. F.; Gylfason, K. B.; Kazmierczak, A.; Dortu, F.; Banuls Polo, M. J.; Maquieira Catala, A.; Kresbach, G. M.; Sohlstrom, H.; Moh, T.; Vivien, L.; Popplewell, J.; Ronan, G.; Barrios, C. A.; Stemme, G.; van der Wijngaart, W. A Packaged Optical Slot-Waveguide Ring Resonator Sensor Array for Multiplex Label-Free Assays in Labs-on-Chips. Lab on a Chip 2010, 10, 281-290.

28. Escorihuela, J.; Marcelis, A. T. M.; Zuilhof, H. Metal-Free Click Chemistry Reactions on Surfaces. Adv. Mater. Interfaces 2015, 2 , 1500135 .

29. Wijdeven, M. A.; Nicosia, C.; Borrmann, A.; Huskens, J.; van Delft, F. L. Biomolecular Patterning of Glass Surfaces Via StrainPromoted Cycloaddition of Azides and Cyclooctynes. RSC Adv. 2014, 4, 10549-10552.

30. Canalle, L. A.; Vong, T.; Adams, P. H. H. M.; van Delft, F. L.; Raats, J. M. H.; Chirivi, R. G. S.; van Hest, J. C. M. Clickable Enzyme-Linked Immunosorbent Assay. Biomacromolecules 2011, 12, 3692-3697.

31. Manova, R. K.; Pujari, S. P.; Weijers, C. A.; Zuilhof, H.; van Beek, T. A. Copper-Free Click Biofunctionalization of Silicon Nitride Surfaces Via Strain-Promoted Alkyne-Azide Cycloaddition Reactions. Langmuir 2012, 28, 8651-8663.

32. Wendeln, C.; Singh, I.; Rinnen, S.; Schulz, C.; Arlinghaus, H. F.; Burley, G. A.; Ravoo, B. J. Orthogonal, Metal-Free Surface Modification by Strain-Promoted Azide-Alkyne and Nitrile Oxide-Alkene/Alkyne Cycloadditions. Chem. Sci. 2012, 3 , 2479-2484.

33. Dijt, J. C.; Stuart, M. A. C.; Fleer, G. J. Reflectometry as a Tool for Adsorption Studies. Adv. Colloid Interfac. 1994, 50, 79-101.

34. Nguyen, A. T.; Baggerman, J.; Paulusse, J. M.; van Rijn, C. J.; Zuilhof, H. Stable Protein-Repellent Zwitterionic Polymer Brushes Grafted from Silicon Nitride. Langmuir 2011, 27, 2587-2594.

35. Chen, L.; Honma, Y.; Mizutani, T.; Liaw, D. J.; Gong, J. P.; Osada, Y. Effects of Polyelectrolyte Complexation on the UCST of Zwitterionic Polymer. Polymer 2000, 41, 141-147.

36. Arafat, A.; Schroën, K.; de Smet, L. C. P. M.; Sudhölter, E. J. R.; Zuilhof, H. Tailor-Made Functionalization of Silicon Nitride Surfaces. J.Am. Chem. Soc. 2004, 126, 8600-8601.

37. Schwarz, S. U.; Cimalla, V.; Eichapfel, G.; Himmerlich, M.; Krischok, S.; Ambacher, O. Thermal Functionalization of GaN Surfaces with 1-Alkenes. Langmuir 2013, 29, 6296-6301.

38. Li, B.; Franking, R.; Landis, E. C.; Kim, H.; Hamers, R. J. Photochemical Grafting and Patterning of Biomolecular Layers onto $\mathrm{TiO}_{2}$ Thin Films. ACS Appl. Mater. Interfaces 2009, 1, 1013-1022.

39. Carvalho, R. R.; Pujari, S. P.; Lange, S. C.; Sen, R.; Vrouwe, E. X.; Zuilhof, H. Local Light-Induced Modification of the inside of Microfluidic Glass Chips. Langmuir 2016, 32, 2389-2398.

40. Prime, K. L.; Whitesides, G. M. Self-Assembled Organic Monolayers - Model Systems for Studying Adsorption of Proteins at Surfaces. Science 1991, 252, 1164-1167.

41. Morag, E.; Bayer, E. A.; Wilchek, M. Reversibility of Biotin-Binding by Selective Modification of Tyrosine in Avidin. Biochem. J. 1996, 316, 193-199.

42. Piran, U.; Riordan, W. J. Dissociation Rate Constant of the Biotin-Streptavidin Complex. J. Immunol. Methods 1990, 133, 141143.

43. Pujari, S. P.; Scheres, L.; Marcelis, A. T. M.; Zuilhof, H. Covalent Surface Modification of Oxide Surfaces. Angew. Chem. Int. Ed. 2014, 53, 6322-6356. 


\subsection{Supporting Information}

\subsubsection{Synthesis of azido-SB (5)}<smiles>CN(CCCN)CCCNCCCNC(=O)OC(C)(C)C</smiles><smiles>C=C(C)C(=O)NCCC[N+](C)(C)CCCN</smiles>

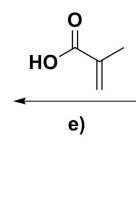

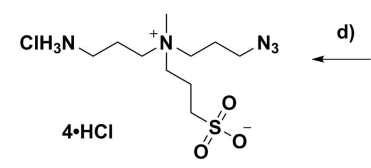<smiles>CC(C)(C)OC(=O)NCCC[N+](C)(CCCN)CCCS(=O)(=O)[O-]</smiles>

Scheme S2.1 Synthesis of azido-SB 5: a) Boc $\mathrm{O}_{2} \mathrm{DCM}, 0{ }^{\circ} \mathrm{C}$ to RT, overnight; b) DTR, $\mathrm{CuSO}_{4}, \mathrm{~K}_{2} \mathrm{CO}_{3}$, $\mathrm{MeOH}$, RT, overnight; c) 3 Bromopropanesulfonic acid sodium salt, DMF, $70{ }^{\circ} \mathrm{C}, 4 \mathrm{~d}$; d) $4 \mathrm{M} \mathrm{HCl}$, Dioxane, 24 h, RT; e) Methacrylic acid, HOBt, EDCI·HCl, NMM, DMF s.g., 2 d, RT.

\section{TERT-BUTYL (3-((3-AMINOPROPYL)(METHYL)AMINO)PROPYL)CARBAMATE 1}

$10.9 \mathrm{~g} \mathrm{Boc}_{2} \mathrm{O}(50 \mathrm{mmol}, 1.00 \mathrm{eq})$ in $500 \mathrm{~mL}$ DCM were dropwise added to a solution of $72.6 \mathrm{~g} \mathrm{~N}$-(3-aminopropyl)-N-methylpropane-1,3-diamine (500 mmol, $10.0 \mathrm{eq}$ ) in $500 \mathrm{~mL}$ DCM over a course of $14 \mathrm{~h}$ at $0{ }^{\circ} \mathrm{C}$. The reaction mixture was allowed to warm up and was stirred for another $12 \mathrm{~h}$ at RT. The white precipitate was filtered off and the filtrate concentrated in vacuum. The crude mixture was diluted with $200 \mathrm{~mL}$ of DCM and washed three times with MilliQ water $(3 \times 100 \mathrm{~mL})$. Drying of the combined organic layer over $\mathrm{Na}_{2} \mathrm{SO}_{4}$ and evaporation of the solvent yielded the product as a colorless oil.<smiles>CN(CCCN)CCCNC(=O)OC(C)(C)C</smiles>

Scheme S2.2 Compound 1 .

$\left[\mathrm{C}_{12} \mathrm{H}_{27} \mathrm{~N}_{3} \mathrm{O}_{2}, \mathrm{M}=245.37 \mathrm{~g} / \mathrm{mol}\right]$, Yield: $10.1 \mathrm{~g}(41.0 \mathrm{mmol}, 82 \%)$

${ }^{1} \mathbf{H}-\mathbf{N M R}\left(400 \mathrm{MHz}, \mathrm{CDCl}_{3}, 300 \mathrm{~K}\right) \delta 5.44(\mathrm{~s}, 1 \mathrm{H}, 4 \mathrm{H}), 3.11\left(\mathrm{q},{ }^{3} \mathrm{JH}_{\mathrm{HH}}=5.6 \mathrm{~Hz}, 2 \mathrm{H}, 5 \mathrm{H}\right), 2.68$ $\left(\mathrm{t},{ }^{3} \mathrm{~J}_{\mathrm{HH}}=6.9 \mathrm{~Hz}, 2 \mathrm{H}, 11 \mathrm{H}\right), 2.32\left(2 \mathrm{t},{ }^{3} \mathrm{~J}_{\mathrm{HH}}=7.0 \mathrm{~Hz}, 4 \mathrm{H}, 7,9 \mathrm{H}\right), 2.13(\mathrm{~s}, 3 \mathrm{H}, 8 \mathrm{H}), 1.56$ 
$\left(2 \mathrm{dt},{ }^{3} \mathrm{~J}_{\mathrm{HH}}=6.8 \mathrm{~Hz}, 4 \mathrm{H}, 6,10 \mathrm{H}\right), 1.38(\mathrm{~s}, 9 \mathrm{H}, 1 \mathrm{H}), 1.33(\mathrm{~s}, 2 \mathrm{H}, 12 \mathrm{H})$.

${ }^{13}$ C-NMR $\left(101 \mathrm{MHz}, \mathrm{CDCl}_{3}\right) \delta 156.1$ (s, C3), 78.8 (s, C2), 56.4 (s, C7), 55.6 (s, C9), 42.1 (s, C8), 40.5 (s, C11), 39.9 (s, C5), 31.1 (s, C10), 28.5 (s, C1), 27.0 (s, C6).

ESI $^{+}(\mathrm{MeOH}):$ calc.: $\mathrm{m} / \mathrm{z}=246.2176[\mathrm{M}+\mathrm{H}]^{+}$; det.: $\mathrm{m} / \mathrm{z}=246.2167[\mathrm{M}+\mathrm{H}]^{+}$.

\section{IMIDAZOLE-1-SULFONYL AZIDE HYDROSULFATE DTR ${ }^{\text {s4 }}$}

$16.1 \mathrm{~mL}$ of sulforyl chloride (200 mmol, 1.0 eq.) was added dropwise to an ice-cold suspension of $13.0 \mathrm{~g} \mathrm{NaN}_{3}$ (200 mmol, 1.0 eq.) in dry $\mathrm{ACN}(100 \mathrm{~mL})$ and the reaction mixture was stirred overnight at RT. $25.9 \mathrm{~g}$ of imidazole (380 mmol, 1.9 eq.) was added in portions and stirring was continued for another $3 \mathrm{~h}$ at RT. Afterwards $200 \mathrm{~mL}$ EtOAc were added before the organic layer was washed with MilliQ water $\left(2 \times 100 \mathrm{~mL}\right.$ and $\mathrm{NaHCO}_{3}(2 \times 100 \mathrm{~mL})$. Drying over $\mathrm{MgSO}_{4}$ and filtration yielded the free imidazole-1-sulfonyl azide. To obtain the sulfuric acid salt, $10.6 \mathrm{~mL}$ of $\mathrm{H}_{2} \mathrm{SO}_{4}(200 \mathrm{mmol}, 1.0$ eq.) were dissolved in $100 \mathrm{~mL}$ of EtOAc and added dropwise to the filtrate. The reaction mixture was stirred for another hour at RT and then placed in the freezer overnight. The resulting crystals were filtered and washed with EtOAc, before being dried to yield the product as white crystals.<smiles>NS(=O)(=O)n1ccnc1S(=O)(=O)O</smiles>

Scheme S2.3 DTR.

$\left[\mathrm{C}_{3} \mathrm{H}_{4} \mathrm{~N}_{5} \mathrm{O}_{6} \mathrm{~S}_{2}, \mathrm{M}=270.21 \mathrm{~g} / \mathrm{mol}\right]$, Yield: $30.2 \mathrm{~g}(111 \mathrm{mmol}, 56 \%)$

${ }^{1} \mathrm{H}-\mathrm{NMR}\left(400 \mathrm{MHz}, \mathrm{DMSO}-\mathrm{d}_{6}\right) \delta 12.05(\mathrm{~s}, 1 \mathrm{H}, 4 \mathrm{H}), 8.938 .82(\mathrm{~m}, 1 \mathrm{H}, 1 \mathrm{H}), 8.03(\mathrm{dd}$, $\left.{ }^{3} \mathrm{~J}_{\mathrm{HH}}=2.0 \mathrm{~Hz},{ }^{4} \mathrm{~J}_{\mathrm{HH}}=1.6 \mathrm{~Hz}, 1 \mathrm{H}, 3 \mathrm{H}\right), 7.45\left(\mathrm{dd},{ }^{3} \mathrm{~J}_{\mathrm{HH}}=2.0 \mathrm{~Hz},{ }^{4} \mathrm{~J}_{\mathrm{HH}}=1.7 \mathrm{~Hz}, 1 \mathrm{H}, 2 \mathrm{H}\right)$.

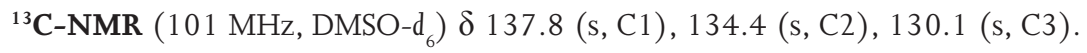

$\mathbf{E S I}^{+}(\mathrm{MeOH}):$ calc.: $\mathrm{m} / \mathrm{z}=174.0080[\mathrm{M}]^{+}$; det.: $\mathrm{m} / \mathrm{z}=174.0080[\mathrm{M}]^{+}$.

\section{TERT-BUTYL (3-((3-AZIDOPROPYL)(METHYL)AMINO)PROPYL)CARBAMATE 2}

$9.57 \mathrm{~g}$ of 1 (39.0 mmol, 1.0 eq.), $10.8 \mathrm{~g}$ of $\mathrm{K}_{2} \mathrm{CO}_{3}$ (78.0 mmol, 2.0 eq.) and $98 \mathrm{mg}$ of $\mathrm{CuSO}_{4}$ (0.39 mmol, $1 \mathrm{~mol} \%$ ) were suspended in $400 \mathrm{~mL} \mathrm{MeOH}$. Subsequently $12.7 \mathrm{~g}$ of DTR (46.8 mmol, 1.2 eq.) was added in portions at $0{ }^{\circ} \mathrm{C}$ and stirred the reaction mixture at RT overnight. After evaporation of $\mathrm{MeOH}$ the crude mixture was dissolved in DCM and water and the organic layer washed three times with MilliQ water. Combined organic layers were dried over $\mathrm{Na}_{2} \mathrm{SO}_{4}$ and concentrated. Flash column chromatography using a gradient from 5-10\% $\mathrm{EtOH}$ in DCM yielded the product as slightly yellow oil. 


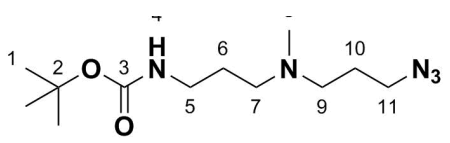

Scheme S2.4 Compound 2 .

$\left[\mathrm{C}_{12} \mathrm{H}_{25} \mathrm{~N}_{5} \mathrm{O}_{2}, \mathrm{M}=271.37 \mathrm{~g} / \mathrm{mol}\right]$, Yield: $6.25 \mathrm{~g}(23.0 \mathrm{mmol}, 59 \%)$

${ }^{1} \mathbf{H}-\mathbf{N M R}\left(400 \mathrm{MHz}, \mathrm{CDCl}_{3}\right) \delta 5.31(\mathrm{~s}, 1 \mathrm{H}, 4 \mathrm{H}), 3.32\left(\mathrm{t},{ }^{3} \mathrm{~J}_{\mathrm{HH}}=6.8 \mathrm{~Hz}, 2 \mathrm{H}, 11 \mathrm{H}\right), 3.15$ (q, $\left.{ }^{3} \mathrm{~J}_{\mathrm{HH}}=6.4 \mathrm{~Hz}, 2 \mathrm{H}, 5 \mathrm{H}\right), 2.47-2.35(\mathrm{~m}, 4 \mathrm{H}, 7,9 \mathrm{H}), 2.19(\mathrm{~s}, 3 \mathrm{H}, 8 \mathrm{H}), 1.73\left(\mathrm{p},{ }^{3} \mathrm{~J}_{\mathrm{HH}}=6.9 \mathrm{~Hz}\right.$, $2 \mathrm{H}, 10 \mathrm{H}), 1.63\left(\mathrm{p},{ }^{3} \mathrm{JH}_{\mathrm{HH}}=6.6 \mathrm{~Hz}, 2 \mathrm{H}, 6 \mathrm{H}\right), 1.41(\mathrm{~s}, 9 \mathrm{H}, 1 \mathrm{H})$.

${ }^{13} \mathrm{C}-\mathrm{NMR}\left(101 \mathrm{MHz}, \mathrm{CDCl}_{3}\right) \delta 156.2$ (C3), 79.0 (C2), 56.3 (C7), 54.7 (C9), 49.5 (C11), 41.8 (C8), 39.8 (C5), 28.5 (C1), 26.8 (C10), 26.6 (C6).

ESI $^{+}(\mathrm{MeOH})$ : calc.: $\mathrm{m} / \mathrm{z}=272.2081[\mathrm{M}+\mathrm{H}]^{+}$; det.: $\mathrm{m} / \mathrm{z}=272.2069[\mathrm{M}+\mathrm{H}]^{+} ; 294.1900$ $[\mathrm{M}+\mathrm{Na}]^{+}$; det.: $\mathrm{m} / \mathrm{z}=294.1888[\mathrm{M}+\mathrm{Na}]^{+}$.

\section{3-((3-AZIDOPROPYL)(3-((TERT-BUTOXYCARBONYL)AMINO)PROPYL) (METHYL)AMMONIO)PROPANE-1-SULFONATE 3}

$4.70 \mathrm{~g}$ of 2 (17.3 mmol, 1.0 eq.) and $3.90 \mathrm{~g}$ of 3-bromopropanesulfonic acid sodium salt (17.3 mmol, 1.0 eq.) were suspended in $100 \mathrm{~mL}$ DMF s.g.. After stirring the reaction mixture at $70{ }^{\circ} \mathrm{C}$ for 2 days, $4.50 \mathrm{~mL}$ DIPEA (26.0 mmol. 1.5 eq.) were added and the suspension was stirred for 1 hour at $70{ }^{\circ} \mathrm{C}$. Another eq. of 3 bromopropanesulfonic acid sodium salt (3.90 g, $17.3 \mathrm{mmol}$ ) was added and the reaction mixture was stirred for $3 \mathrm{~d}$ at $70{ }^{\circ} \mathrm{C}$. After filtering off the formed $\mathrm{NaBr}$, the solvent was evaporated and the crude mixture was dissolved in DCM/ $\mathrm{EtOH}(1: 1)$ and the formed precipitate filtered off over zeolite. The resulting solution was purified by flash column chromatography using a gradient of $10-75 \% \mathrm{EtOH}$ in DCM. The product was isolated as white foam.

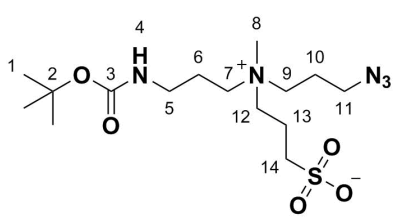

Scheme S2.5 Compound 3.

$\left[\mathrm{C}_{15} \mathrm{H}_{31} \mathrm{~N}_{5} \mathrm{O}_{5} \mathrm{~S}, \mathrm{M}=393.50 \mathrm{~g} / \mathrm{mol}\right]$, Yield: $5.00 \mathrm{~g}(12.7 \mathrm{mmol}, 73 \%)$

${ }^{1} \mathrm{H}-\mathrm{NMR}\left(400 \mathrm{MHz}, \mathrm{DMSO}_{6}\right) \delta 6.96\left(\mathrm{t},{ }^{3} \mathrm{~J}_{\mathrm{HH}}=5.1 \mathrm{~Hz}, 1 \mathrm{H}, 4 \mathrm{H}\right), 3.47\left(\mathrm{t},{ }^{3} \mathrm{~J}_{\mathrm{HH}}=6.5 \mathrm{~Hz}, 2 \mathrm{H}\right.$, $11 \mathrm{H}), 3.44-3.36(\mathrm{~m}, 2 \mathrm{H}, 12 \mathrm{H}), 3.31-3.19(\mathrm{~m}, 4 \mathrm{H}, 7,9-\mathrm{H}), 3.03-2.98(\mathrm{~m}, 2 \mathrm{H}, 5-\mathrm{H})$, $2.97(\mathrm{~s}, 3 \mathrm{H}, 8 \mathrm{H}), 2.47\left(\mathrm{t},{ }^{3} \mathrm{H}_{\mathrm{HH}}=6.9 \mathrm{~Hz}, 2 \mathrm{H}, 14 \mathrm{H}\right), 2.01-1.86(\mathrm{~m}, 4 \mathrm{H}, 10,13 \mathrm{H}), 1.78$ (dt, $\left.{ }^{3} \mathrm{~J}_{\mathrm{HH}}=14.4,6.4 \mathrm{~Hz}, 2 \mathrm{H}, 6 \mathrm{H}\right), 1.38(\mathrm{~s}, 9 \mathrm{H}, 1 \mathrm{H})$. 
${ }^{13}$ C-NMR (101 MHz, DMSO-d $\left.{ }_{6}\right) \delta 155.6$ (C3), 77.8 (C2), 60.2 (C12), 59.1 (C7), 58.2 (C9), 47.8 (C11), 47.7 (C8), 47.5 (C14), 37.0 (C5), 28.2 (C1), 22.2 (C6), 21.4 (C10), 18.5 (C13).

ESI $^{+}(\mathrm{MeOH})$ : calc.: $\mathrm{m} / \mathrm{z}=394.2119[\mathrm{M}+\mathrm{H}]^{+}, 416.1938[\mathrm{M}+\mathrm{Na}]^{+}, 809.3984[2 \mathrm{M}+\mathrm{Na}]^{+}$; det.: $\mathrm{m} / \mathrm{z}=394.2099[\mathrm{M}+\mathrm{H}]^{+}, 416.1918[\mathrm{M}+\mathrm{Na}]^{+}, 809.3942[2 \mathrm{M}+\mathrm{Na}]^{+}$.

\section{3-((3-AMINOPROPYL)(3-AZIDOPROPYL)(METHYL)AMMONIO)PROPANE-1- SULFONATE 4}

$1.44 \mathrm{~g}$ of 3 (3.66 mmol, 1.0 eq.) were suspended in $8.0 \mathrm{~mL}$ dioxane before $4.0 \mathrm{~mL}$ of hydrochloric acid $(4.0 \mathrm{M})$ were added. The resulting solution was stirred overnight at RT. The solvent was removed under vacuum and the resulting viscous compound co-evaporated with water until a slightly yellow solid foam formed.

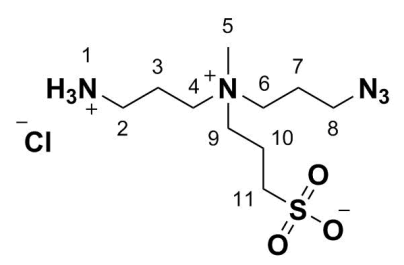

Scheme $\mathbf{S 2 . 6}$ Compound 4 .

$\left[\mathrm{C}_{10} \mathrm{H}_{23} \mathrm{~N}_{5} \mathrm{O}_{3} \mathrm{~S} \cdot \mathrm{HCl}, \mathrm{M}=329.84 \mathrm{~g} / \mathrm{mol}\right]$, Yield: $1.20 \mathrm{~g}(3.66 \mathrm{mmol}, 100 \%)$

${ }^{1} \mathrm{H}-\mathrm{NMR}\left(400 \mathrm{MHz}, \mathrm{DMSO}_{6}\right) \delta 7.90(\mathrm{~s}, 2 \mathrm{H}, 1 \mathrm{H}), 3.49\left(\mathrm{t},{ }^{3} \mathrm{~J}_{\mathrm{HH}}=6.5 \mathrm{~Hz}, 2 \mathrm{H}, 8 \mathrm{H}\right)$, $3.47-3.40(\mathrm{~m}, 2 \mathrm{H}, 9 \mathrm{H}), 3.39-3.32(\mathrm{~m}, 2 \mathrm{H}, 4 \mathrm{H}), 3.32-3.26(\mathrm{~m}, 2 \mathrm{H}, 6 \mathrm{H}), 2.99(\mathrm{~s}, 3 \mathrm{H}$, $5 \mathrm{H}), 2.94-2.83(\mathrm{~m}, 2 \mathrm{H}, 2 \mathrm{H}), 2.54-2.50(\mathrm{~m}, 2 \mathrm{H}, 11 \mathrm{H}), 1.96(\mathrm{~m}, 6 \mathrm{H}, 3,7,10 \mathrm{H})$.

${ }^{13}$ C-NMR (101 MHz, DMSO-d $) ~ \delta 60.1$ (C9), 58.7 (C4), 57.8 (C6), 47.8 (C8), 47.7 (C5), 47.4 (C11), 36.1 (C2), 21.5 (C3), 20.1 (C7), 18.5 (C10).

ESI $^{+}(\mathrm{MeOH}):$ calc.: $\mathrm{m} / \mathrm{z}=294.1594[\mathrm{M}+\mathrm{H}]^{+}, 587.3116[2 \mathrm{M}+\mathrm{H}]^{+}$; det.: $\mathrm{m} / \mathrm{z}=294.1582$ $[\mathrm{M}+\mathrm{H}]^{+}, 587.3093[2 \mathrm{M}+\mathrm{H}]^{+}$.

\section{3-((3-AZIDOPROPYL)(3-METHACRYLAMIDOPROPYL)(METHYL)AMMONIO) PROPANE-1-SULFONATE 5}

$557 \mathrm{mg}$ of HOBt (4.12 mmol, $1.2 \mathrm{eq}$ ), $790 \mathrm{mg}$ of EDCI·HCl (4.12 mmol, 1.2 eq.), $1.45 \mathrm{ml}$ of NMM (10.3 mmol, 3.0 eq.) and $0.35 \mathrm{~mL}$ of methacrylic acid (4.12 mmol, 1.2 eq.) were dissolved in $5 \mathrm{~mL}$ of DMF s.g. and stirred at RT. After 2 h, $1.13 \mathrm{~g}$ of 4 (3.43 mmol, 1.0 eq.) dissolved in $2 \mathrm{~mL}$ of MilliQ water were added, and the resulting yellow solution was stirred for $3 \mathrm{~d}$ at RT. The DMF was removed in vacuo, the obtained crude dissolved in $40 \mathrm{~mL}$ of MilliQ water and extracted with EtOAC $(4 \times 50 \mathrm{~mL})$. The aqueous phase was concentrated under high vacuum at RT and the collected product purified by column chromatography. Starting 
the column with DCM : EtOH $(1: 1)$ elutes all coupling reagents and side products, while flushing afterwards with $\mathrm{MeOH}(100 \%)$ isolates the product containing impurities $(\mathrm{MeOH}$ was removed using a water bath at $25{ }^{\circ} \mathrm{C}$ at 50 mbar). A second column starting directly with $\mathrm{MeOH}\left(100 \%, \mathrm{R}_{\mathrm{f}}=0.3\right)$ yielded the pure product as a slightly yellow foam $(986 \mathrm{mg}$, $2.73 \mathrm{mmol}, 80 \%$ ), which allows for the modification of $2701 \times 1 \mathrm{~cm}$ substrates with a CO- $(5)_{1 \%}$-polymer brush. In order to prevent spontaneous polymerization during storage, the product was dissolved in water and proportioned in twenty equal fractions that were stored frozen at $-80^{\circ} \mathrm{C}$ prior to use for surface modification.

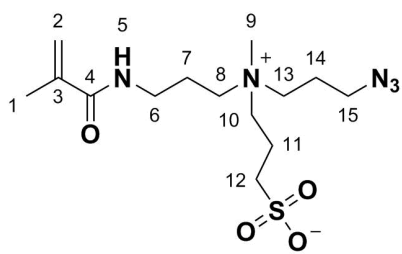

Scheme S2.7 Compound 5 .

$\left[\mathrm{C}_{14} \mathrm{H}_{27} \mathrm{~N}_{5} \mathrm{O}_{4} \mathrm{~S}, \mathrm{M}=361.46 \mathrm{~g} / \mathrm{mol}\right]$, Yield: $986 \mathrm{mg}(2.73 \mathrm{mmol}, 80 \%)$

${ }^{1} \mathrm{H}-\mathbf{N M R}\left(400 \mathrm{MHz}, \mathrm{DMSO}-d_{6}\right) \delta 8.05\left(\mathrm{t},{ }^{3}{ }_{\mathrm{HH}}=5.7 \mathrm{~Hz}, 1 \mathrm{H}, 5 \mathrm{H}\right), 5.67\left(\mathrm{~s}, 1 \mathrm{H}, 2_{\mathrm{a}} \mathrm{H}\right), 5.34$ $\left(\mathrm{s}, 1 \mathrm{H}, 2 \mathrm{~b}_{\mathrm{b}} \mathrm{H}\right), 3.47\left(\mathrm{t},{ }^{3}{ }_{\mathrm{HH}}=6.5 \mathrm{~Hz}, 2 \mathrm{H}, 15 \mathrm{H}\right), 3.443 .38(\mathrm{~m}, 2 \mathrm{H}, 10 \mathrm{H}), 3.313 .23(\mathrm{~m}$, $4 \mathrm{H}, 8,13 \mathrm{H}), 3.20\left(\mathrm{t},{ }^{3}{ }_{\mathrm{HH}}=6.0 \mathrm{~Hz}, 2 \mathrm{H}, 6 \mathrm{H}\right), 2.97(\mathrm{~s}, 3 \mathrm{H}, 9 \mathrm{H}), 2.47\left(\mathrm{t},{ }^{3} \mathrm{JHH}_{\mathrm{HH}}=6.8 \mathrm{~Hz}, 2 \mathrm{H}\right.$, $12 \mathrm{H}), 2.001 .89(\mathrm{~m}, 4 \mathrm{H}, 11,14 \mathrm{H}), 1.881 .81(\mathrm{~m}, 5 \mathrm{H}, 1,7 \mathrm{H})$.

${ }^{13}$ C-NMR (101 MHz, DMSO-d $) \delta 167.7$ (C4), 139.8 (C3), 119.3 (C2), 60.0 (C10), 59.1 (C8), 58.3 (C13), 47.8 (C15), 47.7 (C9), 47.5 (C12), 35.9 (C6), 22.0 (C7), 21.5 (C14), 18.6 (C1), 18.5 (C11).

ESI $^{+}(\mathrm{MeOH}):$ calc.: $\mathrm{m} / \mathrm{z}=362.1857[\mathrm{M}+\mathrm{H}]^{+}, 384.1676[\mathrm{M}+\mathrm{Na}]^{+}$; det.: $\mathrm{m} / \mathrm{z}=362.1833$ $[\mathrm{M}+\mathrm{H}]^{+}, 384.1656[\mathrm{M}+\mathrm{Na}]^{+}$.

\subsubsection{Synthesis of SB}

\section{3-((3-METHACRYLAMIDOPROPYL)DIMETHYLAMMONIO)PROPANE-1-}<smiles>C=C(C)C(=O)NCCCN(C)CCC[N+](C)(C)CCCNC(=O)C(=C)C</smiles>

Scheme S2.8 Synthesis of SB: a) N-(3-(di-methylamino)propyl)methacrylamide, 1,2-propane-sultone, acetone, RT, overnight, $91 \%$. 


\section{SULFONATE (SB) ${ }^{\text {S1 }}$}

$12.2 \mathrm{~g}$ of 1,3-propanesultone (100 mmol, 1.8 eq.) were dissolved in $150 \mathrm{~mL}$ acetone and cooled to $0{ }^{\circ} \mathrm{C}$. After addition of $10.0 \mathrm{~mL}$ of $\mathrm{N}$-(3-(dimethylamino)propyl)methacrylamide ( $55.0 \mathrm{mmol}, 1.0$ eq.) the reaction mixture was allowed to warm up to room temperature and stirred overnight. The resulting precipitate was filtered off and washed rigorously with $500 \mathrm{~mL}$ acetone. After drying under high vacuum the desired product was obtained as white powder and stored under argon in the fridge.

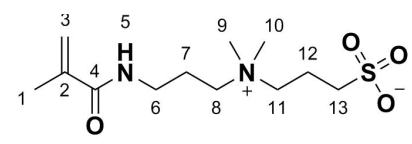

Scheme S2.9 SB monomer.

$\left[\mathrm{C}_{12} \mathrm{H}_{24} \mathrm{~N}_{2} \mathrm{O}_{4} \mathrm{~S}, \mathrm{M}=292.39 \mathrm{~g} / \mathrm{mol}\right]$, Yield: $14.7 \mathrm{~g}(50.0 \mathrm{mmol}, 91 \%)$

${ }^{1} \mathrm{H}-\mathbf{N M R}\left(400 \mathrm{MHz}, \mathrm{D}_{2} \mathrm{O}\right) \delta 5.68(\mathrm{~s}, 1 \mathrm{H}, 3 \mathrm{a}-\mathrm{H}), 5.44(\mathrm{~s}, 1 \mathrm{H}, 3 \mathrm{~b}-\mathrm{H}), 3.49-3.40(\mathrm{~m}, 2 \mathrm{H}, 11$ $\mathrm{H}), 3.38-3.29(\mathrm{~m}, 4 \mathrm{H}, 6,8-\mathrm{H}), 3.08(\mathrm{~s}, 6 \mathrm{H}, 9,10-\mathrm{H}), 2.94\left(\mathrm{t},{ }^{3} \mathrm{~J}_{\mathrm{HH}}=7.2 \mathrm{~Hz}, 2 \mathrm{H}, 13-\mathrm{H}\right)$, $2.24-2.11(\mathrm{~m}, 2 \mathrm{H}, 12-\mathrm{H}), 2.07-1.96(\mathrm{~m}, 2 \mathrm{H}, 7-\mathrm{H}), 1.90(\mathrm{~s}, 3 \mathrm{H}, 1-\mathrm{H})$.

${ }^{13}$ C-NMR (101 MHz, D O) $\delta 171.95$ (C4), 138.76 (C2), 121.33 (C3), 62.12 (C11), 61.81 (C8), 50.68 (C9,10), 47.14 (C13), 36.15 (C6), 22.21 (C7), 18.08 (C12), 17.57 (C1).

ESI $^{+}(\mathrm{MeOH})$ : calc.: $\mathrm{m} / \mathrm{z}=293.1530[\mathrm{M}+\mathrm{H}]^{+}, 315.1349[\mathrm{M}+\mathrm{Na}]^{+}, 585.2986[2 \mathrm{M}+\mathrm{H}]^{+}$, $607.2806[2 \mathrm{M}+\mathrm{Na}]^{+} ;$det.: $\mathrm{m} / \mathrm{z}=293.1515[\mathrm{M}+\mathrm{H}]^{+}, 315.1332[\mathrm{M}+\mathrm{Na}]^{+}, 585.2956[2 \mathrm{M}+\mathrm{H}]^{+}$, $607.2774[2 \mathrm{M}+\mathrm{Na}]^{+}$.

\subsubsection{Synthesis of TFAAD}
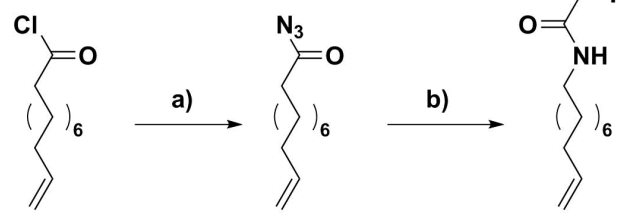

TFAAD

Scheme S2.10 Synthesis of TFAAD: a) 10-undecenoyl chloride, $4 \mathrm{M} \mathrm{NaN}{ }_{3}, \mathrm{TBAB}, 0^{\circ} \mathrm{C}$, overnight; b) TFA, DCM, RT > reflux, overnight, $72 \%$ over two steps.

\section{TRIFLUOROACETAMIDE-PROTECTED 10-AMINO-1-DECENE TFAAD $\mathbf{6}^{\mathbf{5 4 5}}$}

$66 \mathrm{mg}$ of tetrabutylammonium bromide $(0.25 \mathrm{mmol}$, cat.) and $12.16 \mathrm{~g}$ of 10 -undecenoyl 
chloride (60.0 mmol, 1.0 eq.) were combined and cooled to $-3{ }^{\circ} \mathrm{C}$. Aqueous sodium azide solution (3.90 g in $15 \mathrm{~mL}$ MilliQ water) was added dropwise, keeping the temperature below $5{ }^{\circ} \mathrm{C}$. The reaction mixture was stirred for $24 \mathrm{~h}$ at $0{ }^{\circ} \mathrm{C}$. After addition of $15 \mathrm{~mL}$ of cold DCM, the organic phase was washed two times with $15 \mathrm{~mL}$ of cold MilliQ water and dried over $\mathrm{Na}_{2} \mathrm{SO}_{4}$. During the whole workup process solvents and solutions were kept as cool as possible. The resulting acyl azide was directly reacted with $6.0 \mathrm{~mL}$ trifluoroacetic acid (78 mmol, 1.30 eq.). The reaction mixture was allowed to warm up, stirred at RT until gas formation had stopped and then refluxed at $40{ }^{\circ} \mathrm{C}$ overnight. Washing three times with $15 \mathrm{~mL}$ of saturated sodium bicarbonate solution, drying over sodium sulfate and removal of the solvent under reduced pressure gave the crude product. Purification was achieved by column chromatography $(100 \%$ $\left.\mathrm{DCM} ; \mathrm{R}_{\mathrm{f}}=0.8\right)$ and Kugelrohr distillation $\left(0.4 \mathrm{mbar}, 176{ }^{\circ} \mathrm{C}\right)$, which yielded the desired product with a purity of $98 \%$ (GC-MS).

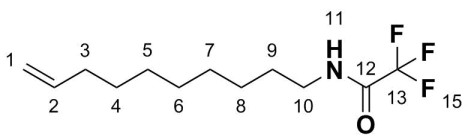

Scheme S2.1 1 TFAAD

$\left[\mathrm{C}_{12} \mathrm{H}_{20} \mathrm{~F}_{3} \mathrm{NO}, \mathrm{M}=251.29 \mathrm{~g} / \mathrm{mol}\right]$, Yield: $10.8 \mathrm{~g}$ (43.1 mmol, $72 \%$ over two steps)

${ }^{1} \mathrm{H}$-NMR $\left(400 \mathrm{MHz}, \mathrm{CDCl}_{3}\right) \delta 6.54(\mathrm{~s}, 1 \mathrm{H}, 11 \mathrm{H}), 5.79\left(\mathrm{ddt},{ }^{3}{ }_{\mathrm{HH}}=16.9,10.2,6.7 \mathrm{~Hz}, 1 \mathrm{H}\right.$, $2 \mathrm{H}), 5.02-4.89(\mathrm{~m}, 2 \mathrm{H}, 1 \mathrm{H}), 3.34\left(\mathrm{q},{ }^{3}{ }_{\mathrm{HH}}=6.8 \mathrm{~Hz}, 2 \mathrm{H}, 10 \mathrm{H}\right), 2.03\left(\mathrm{q},{ }^{3}{ }_{\mathrm{HH}}=7.0 \mathrm{~Hz}\right.$, $2 \mathrm{H}, 3 \mathrm{H}), 1.57\left(\mathrm{p}^{3}{ }_{\mathrm{HH}}=7.1 \mathrm{~Hz}, 2 \mathrm{H}, 9 \mathrm{H}\right), 1.42-1.20(\mathrm{~m}, 10 \mathrm{H}, 4,5,6,7,8 \mathrm{H})$.

${ }^{13}$ C-NMR $\left(101 \mathrm{MHz}, \mathrm{CDCl}_{3}\right) \delta 157.4\left(\mathrm{q},{ }^{2} \mathrm{~J}_{\mathrm{CF}}=36.6 \mathrm{~Hz}, \mathrm{C} 12\right), 139.2(\mathrm{C} 2), 115.9$ (q, $\left.{ }^{1} J_{\mathrm{CF}}=187.9 \mathrm{~Hz}, \mathrm{C} 13\right), 114.6$ (C1), 40.1 (C10), 33.9 (C3), 29.4- 29.0 (C4,5,6,7,9), 26.8 (C8).

ESI $^{+}(\mathrm{MeOH}):$ calc.: $\mathrm{m} / \mathrm{z}=252.1570[\mathrm{M}+\mathrm{H}]^{+}, 274.1389[\mathrm{M}+\mathrm{Na}]^{+}$; det.: $\mathrm{m} / \mathrm{z}=252.1562$ $[\mathrm{M}+\mathrm{H}]^{+}, 274.1379[\mathrm{M}+\mathrm{Na}]^{+}$.
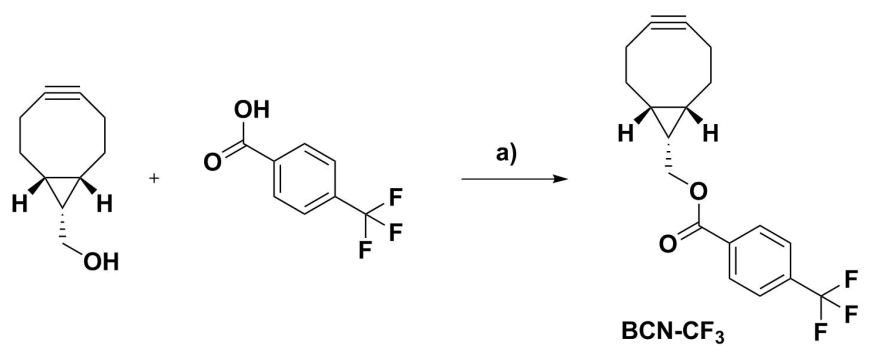

Scheme S2.12 Synthesis of BCN-CF $:$ a) DCC, DMAP, RT, overnight. 


\subsubsection{Synthesis of $\mathrm{BCN}_{-} \mathrm{CF}_{3}$}

$100 \mathrm{mg}$ of (1R,8S,9s)-bicyclo[6.1.0]non-4-yn-9-ylmethanol (0.67 mmol, 1.0 eq.), $139 \mathrm{mg}$ of 4(trifluoromethyl)-benzoic acid (0.73 mmol, 1.1 eq.), $151 \mathrm{mg}$ of DCC (0.73 mmol, 1.1 eq.) and DMAP (catalytic amount) were dissolved in $2 \mathrm{~mL} \mathrm{DCM}$ and stirred overnight at

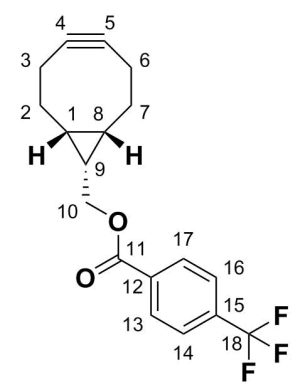

Scheme $\mathbf{S 2 . 1 3} \quad \mathrm{BCN}-\mathrm{CF}_{3}$

RT. After filtration and evaporation of DCM, the crude was purified by column chromatography (EtOAc:heptane $\left.(1: 5), \mathrm{R}_{\mathrm{f}}=0.8\right)$ yielding the product as white solid, which was stored in the freezer $\left(-20^{\circ} \mathrm{C}\right)$.

$\left[\mathrm{C}_{18} \mathrm{H}_{17} \mathrm{~F}_{3} \mathrm{O}_{2}, \mathrm{M}=322.33 \mathrm{~g} / \mathrm{mol}\right]$, Yield: $169 \mathrm{mg}(0.53 \mathrm{mmol}, 79 \%)$

${ }^{1} \mathrm{H}$ NMR $\left.(400 \mathrm{MHz}, \text { DMSO-d })_{6}\right) \delta .17\left(\mathrm{~d},{ }^{3} \mathrm{~J}_{\mathrm{HH}}=8.1 \mathrm{~Hz}, 2 \mathrm{H}, 13,17 \mathrm{H}\right), 7.71\left(\mathrm{~d},{ }^{3} \mathrm{~J}_{\mathrm{HH}}=8.2 \mathrm{~Hz}\right.$, $2 \mathrm{H}, 14,16 \mathrm{H}), 4.46\left(\mathrm{~d},{ }^{3} \mathrm{~J}_{\mathrm{HH}}=8.3 \mathrm{~Hz}, 2 \mathrm{H}, 10 \mathrm{H}\right), 2.39-2.16(\mathrm{~m}, 6 \mathrm{H}, 2,3,6 \mathrm{H}), 1.74-1.59(\mathrm{~m}$, $2 \mathrm{H}, 7 \mathrm{H}), 1.58-1.48(\mathrm{~m}, 1 \mathrm{H}, 9 \mathrm{H}), 1.11-0.96(\mathrm{~m}, 2 \mathrm{H}, 1,8 \mathrm{H})$.

${ }^{13}$ C NMR $\left(101 \mathrm{MHz}, \mathrm{DMSO}-d_{6}\right) \delta 165.64$ (C11), 133.86 (C12), 130.12 (C13,17), $125.61-$ 125.50 (C14,15,16), 98.92 (C4,5), 63.86 (C10), 29.34 (C2,3,6), 21.57 (C7), 20.58 (C1,8), 17.60 (C9).

ESI $^{+}(\mathrm{MeOH}):$ calc.: $\mathrm{m} / \mathrm{z}=323.1259[\mathrm{M}+\mathrm{H}]^{+}, 345.1078[\mathrm{M}+\mathrm{Na}]^{+} ;$det.: $\mathrm{m} / \mathrm{z}=323.1242$ $[\mathrm{M}+\mathrm{H}]^{+}, 345.1061[\mathrm{M}+\mathrm{Na}]^{+}$. 


\subsubsection{Additional XPS data}
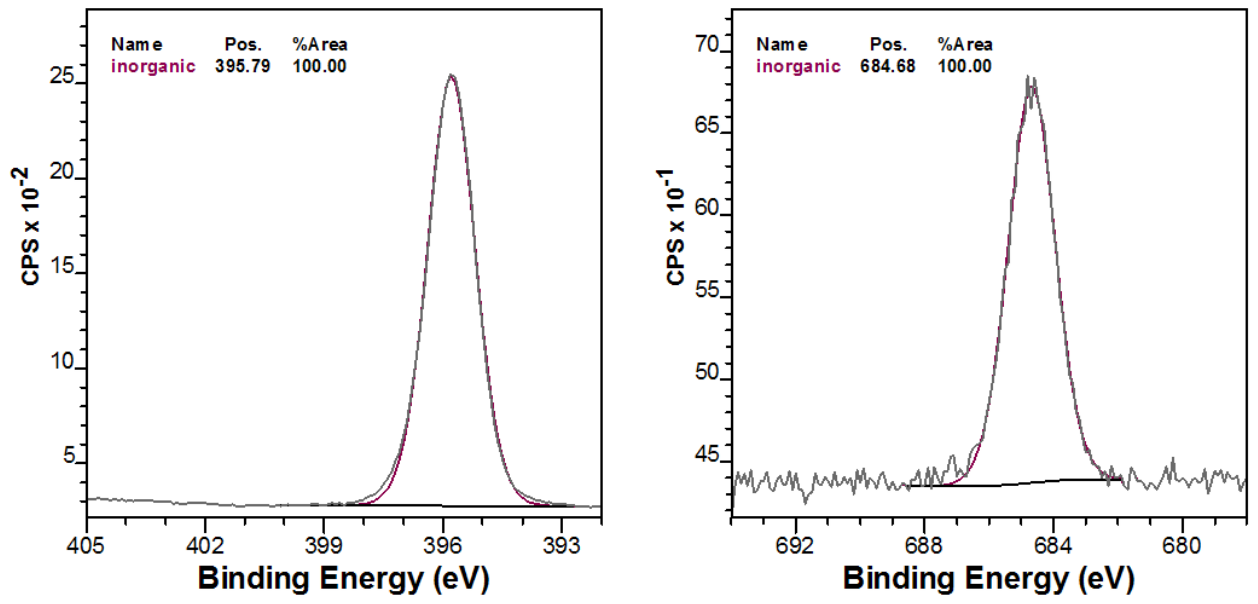

Figure S2.1 XPS data of bare $\mathrm{Si}_{3} \mathrm{~N}_{4}$ : (left) N 1s narrow scan and (right) F 1s narrow scan.
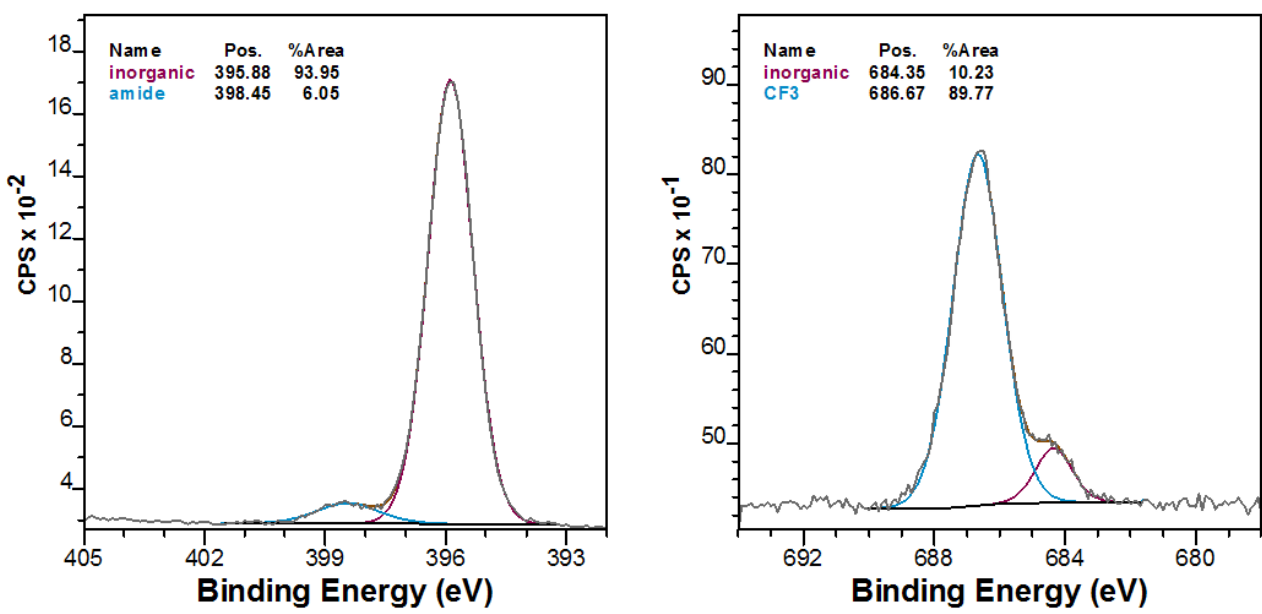

Figure S2.2 XPS data of TFAAD-modified $\mathrm{Si}_{3} \mathrm{~N}_{4}$ : (left) N 1s narrow scan and (right) F 1s narrow scan. 

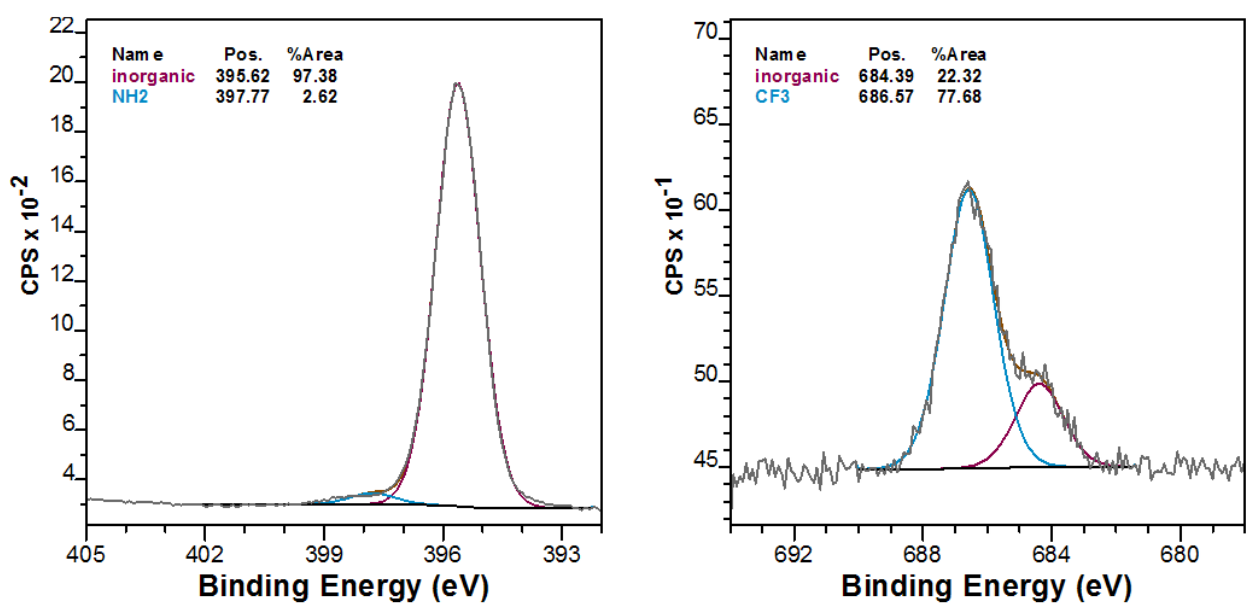

Figure S2.3 XPS data of amine-modified $\mathrm{Si}_{3} \mathrm{~N}_{4}$ : (left) N 1s narrow scan and (right) F 1s narrow scan.

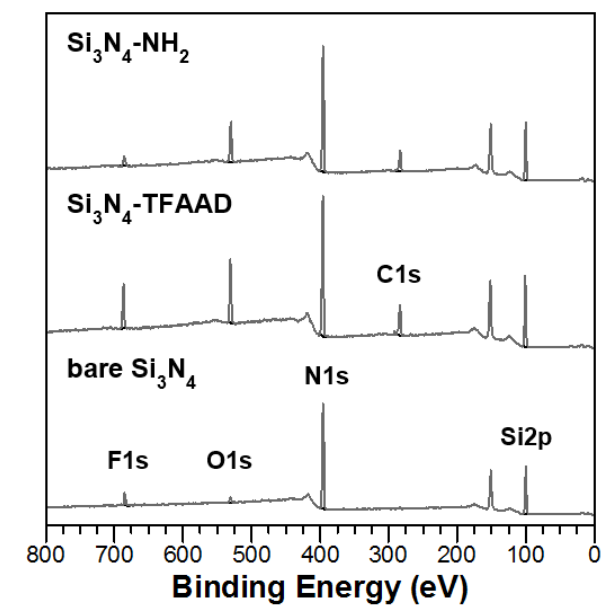

Figure S2.4 Comparison of XPS wide scans of bare $\mathrm{Si}_{3} \mathrm{~N}_{4}$, Si3N4-TFAAD and $\mathrm{Si}_{3} \mathrm{~N}_{4}-\mathrm{NH}_{2}$. 

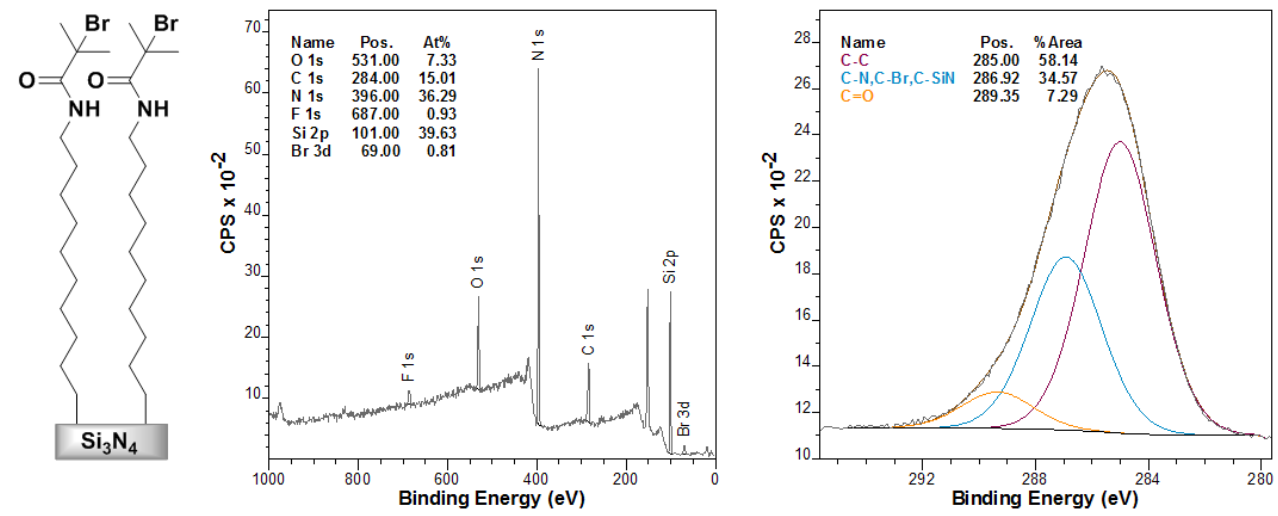

Figure S2.5 (left) schematic representation of initiator-modified $\mathrm{Si}_{3} \mathrm{~N}_{4}$ and XPS data of initiator-coated Si3N4: (middle) wide scan and (right) C 1s narrow scan.
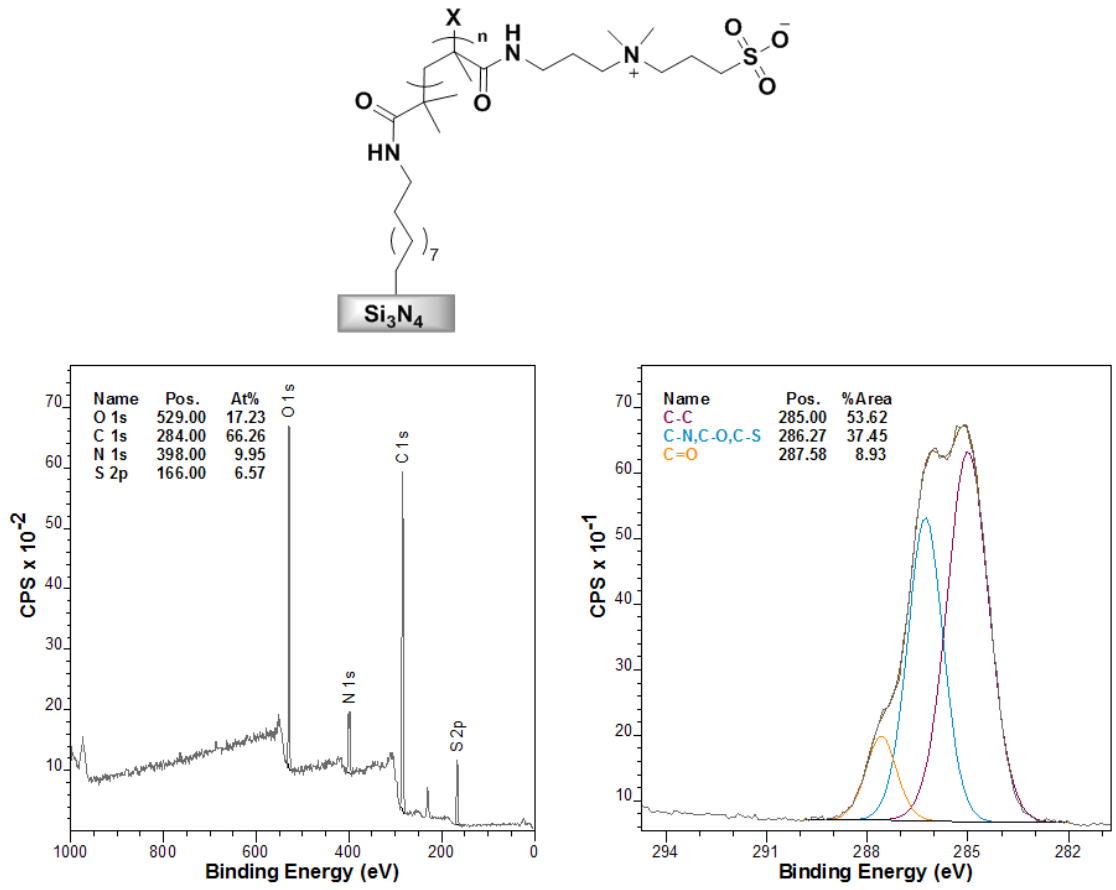

Figure S2.6 (top) schematic representation of $\mathrm{pSB}$ on $\mathrm{Si}_{3} \mathrm{~N}_{4}$ and (bottom) XPS data: (left) wide scan and (right) C 1s narrow scan. 

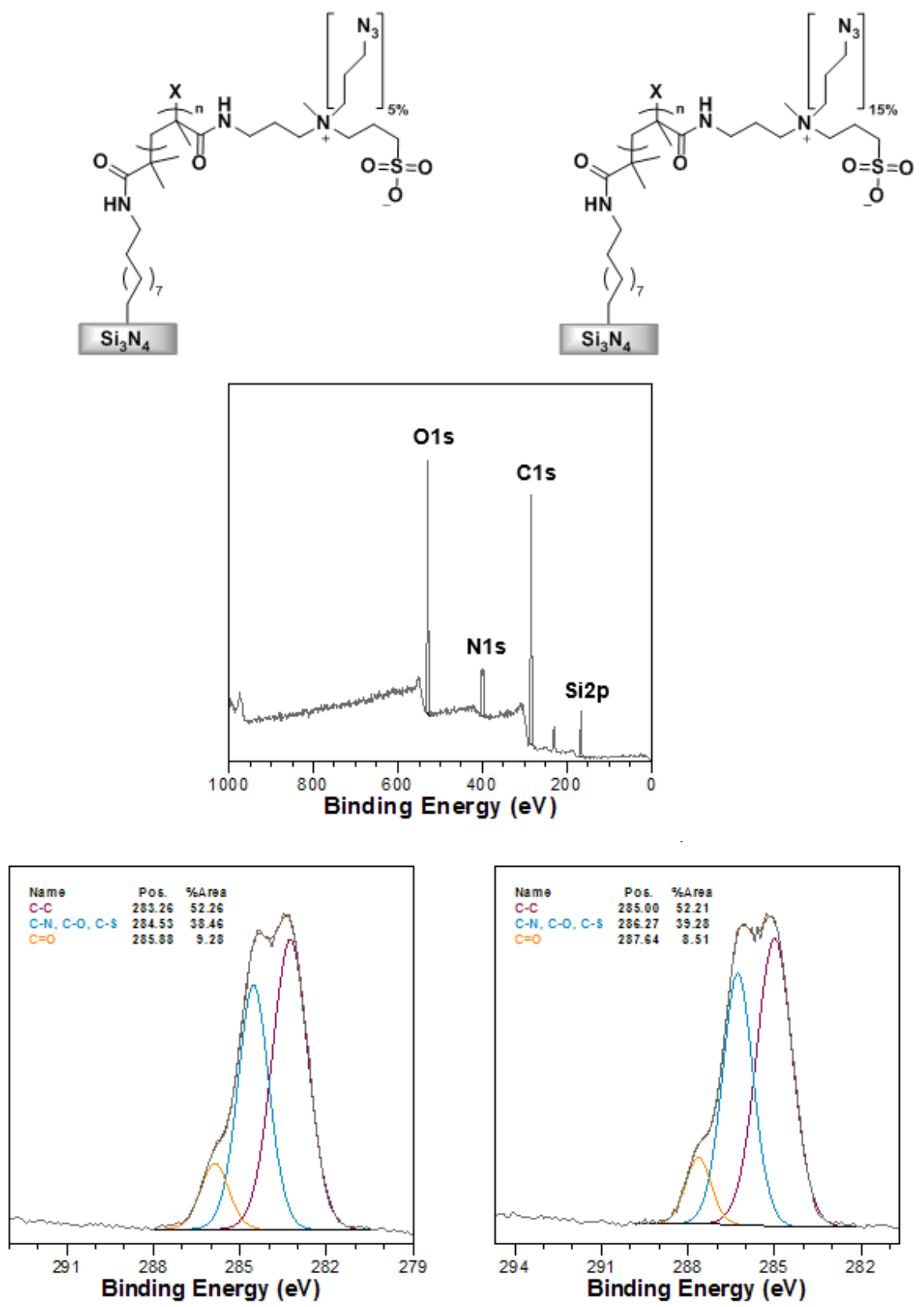

Figure S2.7 (top) schematic representation of co-5 ${ }_{5 \%}{ }^{-}$and co-5 $\mathbf{5}_{15 \%}$-polymer brushes on $\mathrm{Si}_{3} \mathrm{~N}_{4}$ with (middle) characteristic XPS wide scan and (bottom) XPS C 1s narrow scan spectra, respectively. 

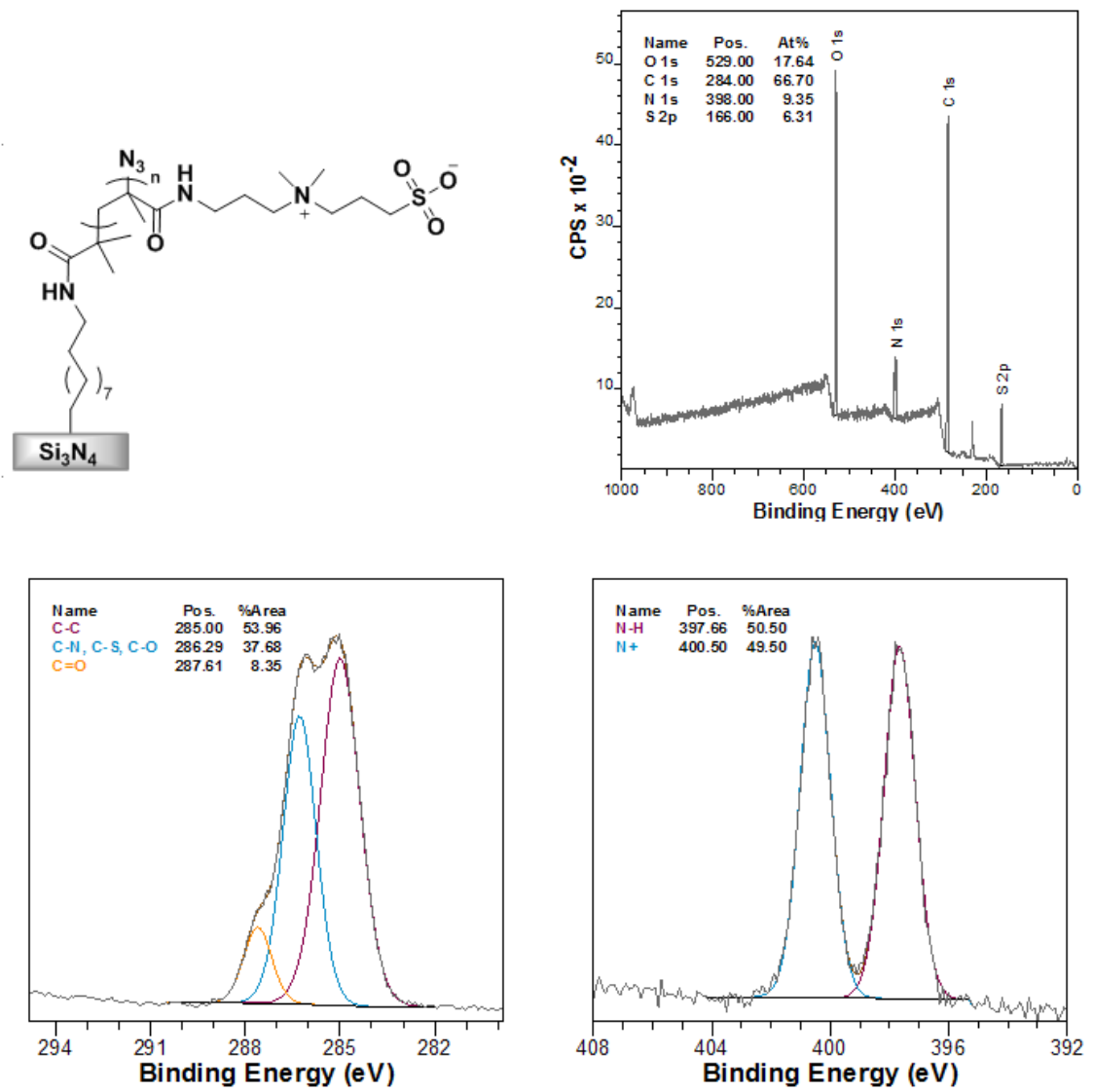

Figure S2.8 (top left) schematic representation of $\mathrm{pSB}-\mathrm{N}_{3}$ polymer brush on $\mathrm{Si}_{3} \mathrm{~N}_{4}$; (top right) XPS wide scan, (bottom left) XPS C 1s narrow scan and (bottom right) XPS N 1s narrow scan. 

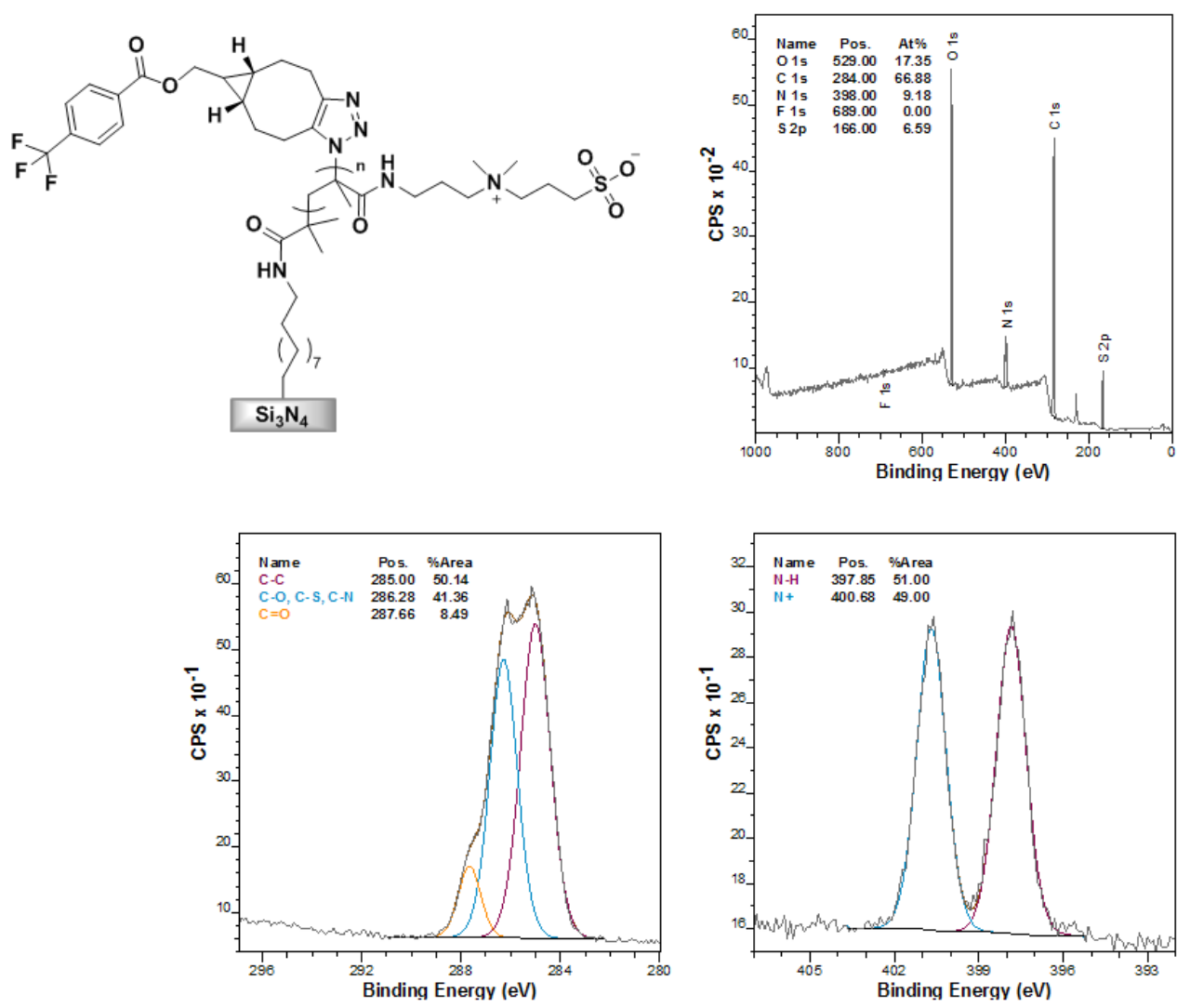

Figure S2.9 top left) schematic representation of $\mathrm{pSB}-\mathrm{CF}_{3}$ polymer brush on $\mathrm{Si}_{3} \mathrm{~N}_{4}$; (top right) XPS wide scan, (bottom left) XPS C 1s narrow scan and (bottom right) XPS N 1s narrow scan. 

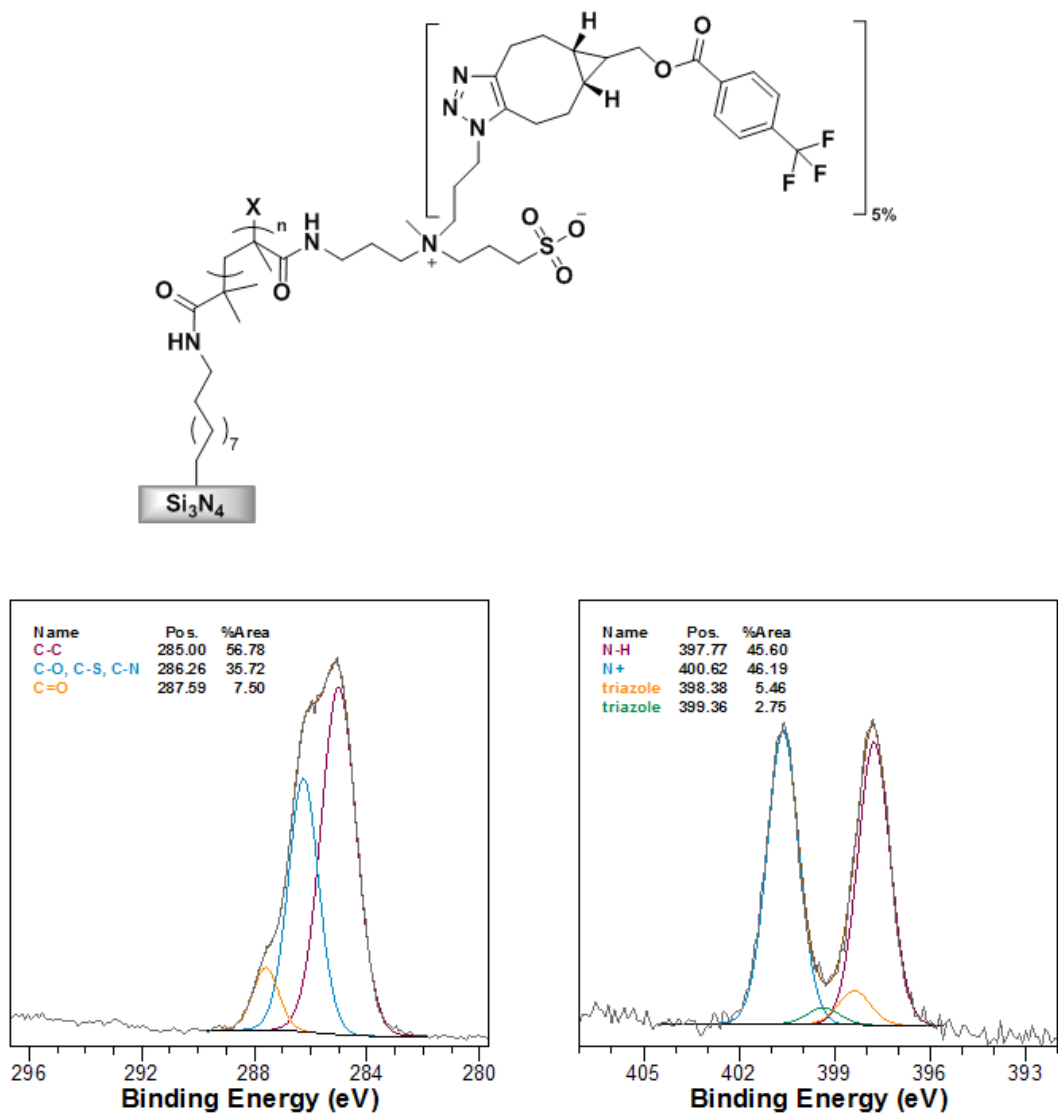

Figure S2.10 top) schematic representation of co- $\left(5-\mathrm{CF}_{3}\right)_{5 \%}$ polymer brushes on $\mathrm{Si}_{3} \mathrm{~N}_{4}$ and (bottom) XPS data of: (left) $\mathrm{C} 1 \mathrm{~s}$ narrow scan and (right) $\mathrm{N}$ 1s narrow scan. 


\subsubsection{SCA and thicknesses of polymer brushes}

\begin{tabular}{|c|c|c|c|}
\hline & standard brush & azide-modified brush & biotin-modified brush \\
\hline$p S B$ & $p S B 14 \pm 1^{\circ}$ & $p S B-N_{3} 17 \pm 1^{\circ}$ & $p$ SB-biotin $27 \pm 2^{\circ}$ \\
\hline co- $5_{1 \%}$ & & Co- $5_{1 \% 20 \pm 2^{\circ}}$ & co-(5-biotin) $)_{1 \%} 31 \pm 2^{\circ}$ \\
\hline co-5 $5 \%$ & & Co- $55 \% 23 \pm 2^{\circ}$ & co-(5-biotin) $5 \% 60 \pm 2^{\circ}$ \\
\hline
\end{tabular}

Table S2.1 Pictures and obtained values of static water contact angle measurements of polymer brushes possessing different azide content. top: pSB, top-functionalized pSB-N $\mathrm{N}_{3}$ and pSB-biotin; middle: co-5 $\mathbf{5}_{1 \%}$ and co-(5-biotin $)_{1 \%}$; bottom: co-55\% and co-(5-biotin $)_{5 \%}$.

\begin{tabular}{|l|l|l|l|l|l|l|l|l|l|}
\hline & \multicolumn{3}{|l|}{ fibrinogen assay } & \multicolumn{3}{l|}{ avidin assay } & \multicolumn{3}{l|}{ protein mix assay } \\
\hline pSB-biotin & 39.4 & 38.0 & 43.2 & 43.9 & 44.1 & 36.7 & & & \\
\hline co-(5-biotin) ${ }_{1 \%}$ & 65.8 & 65.6 & 64.1 & 63.0 & 67.7 & 65.6 & 68.8 & 69.3 & 67.5 \\
\hline co-(5-biotin) & & & & & & & & & \\
\hline
\end{tabular}

Table S2.2 Polymer brush thicknesses of reference pSB and biotin functionalized copolymer brushes used for reflectometry calculations. Thicknesses are recorded in $\mathrm{nm}$. 


\subsubsection{XRR Scattering Profiles of Functionalized Polymer Brushes}

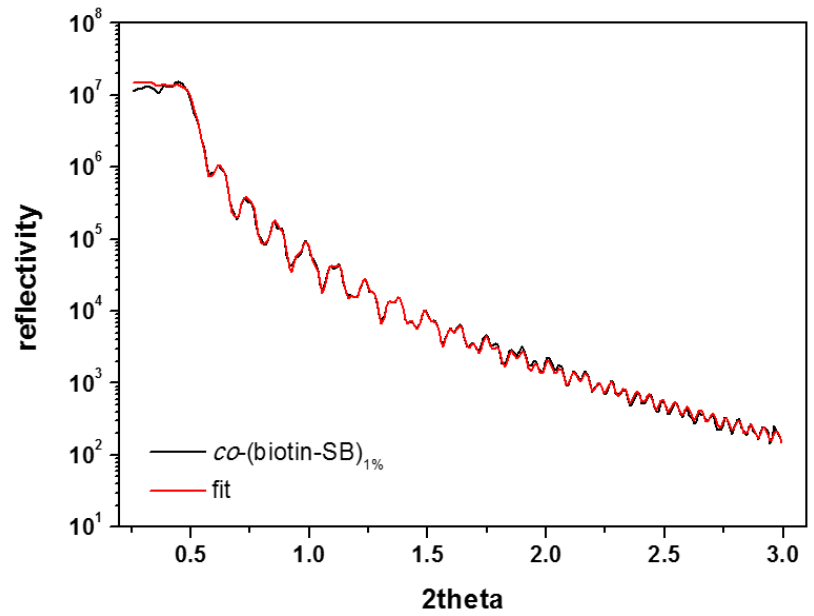

Figure S2.11 Exemplary X-Ray reflectivity scattering profile of co-(biotin-SB) ${ }_{1 \%}$ polymer brush on $\mathrm{Si}_{3} \mathrm{~N}_{4}$ with fit.

\subsection{References}

(S1) Goddard-Borger, E. D.; Stick, R. V. An Efficient, Inexpensive, and Shelf-Stable Diazotransfer Reagent: Imidazole-1sulfonyl Azide Hydrochloride. Org. Lett. 2007, 9, 3797-3800.

(S2) Chen, L.; Honma, Y.; Mizutani, T.; Liaw, D. J.; Gong, J. P.; Osada, Y. Effects of polyelectrolyte complexation on the UCST of zwitterionic polymer. Polymer 2000, 41, 141-147.

(S3) Veliscek-Carolan, J.; Jolliffe, K. A.; Hanley, T. L. Selective Sorption of Actinides by Titania Nanoparticles Covalently Functionalized with Simple Organic Ligands. ACS Appl. Mater. Interfaces 2013, 5, 11984-11994.

(S4) Dijt, J. C.; Stuart, M. A. C.; Fleer, G. J. Reflectometry as a Tool for Adsorption Studies. Adv. Colloid Interfac. 1994, 50, 79101.

(S5) Nguyen, A. T.; Baggerman, J.; Paulusse, J. M.; van Rijn, C. J.; Zuilhof, H. Stable Protein-Repellent Zwitterionic Polymer Brushes Grafted from Silicon Nitride. Langmuir 2011, 27, 2587-2594. 


\section{Chapter.}

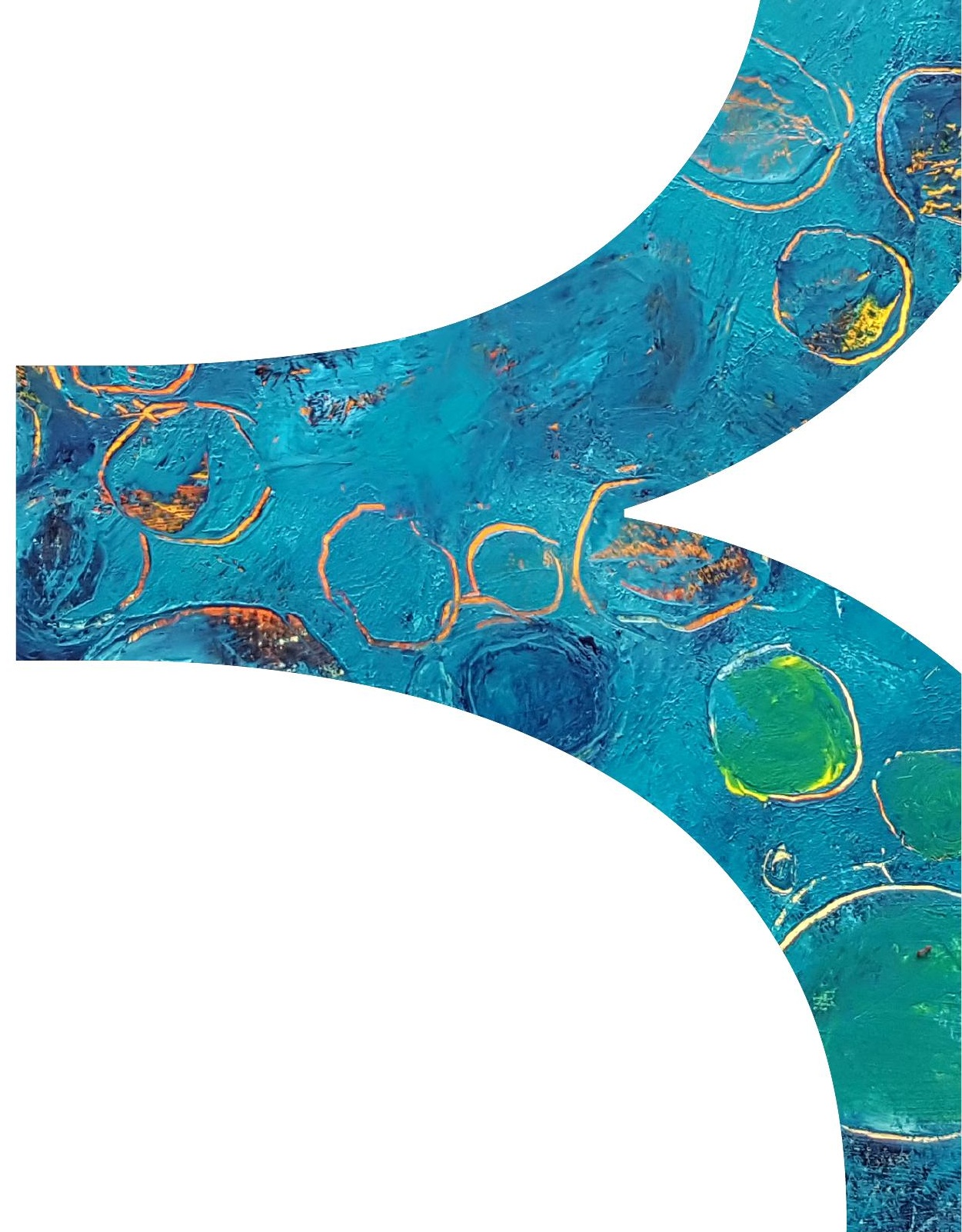




\section{Highly specific}

binding on antifouling zwitterionic polymercoated microbeads as measured by flow cytometry

van Andel, E.; de Bus, I.; Tijhaar, E. J.; Smulders, M. M. J.; Savelkoul, H. F. J.; Zuilhof, H. Highly Specific Binding on Antifouling Zwitterionic Polymer-Coated Microbeads as Measured by Flow Cytometry. ACS Appl. Mater. Interfaces 2017, 9, 38211-38221. 


\begin{abstract}
Micro- and nano-sized particles are extensively used in various biomedical applications. However, their performance is often drastically hampered by the non-specific adsorption of biomolecules, a process called biofouling. Although antifouling coatings have been extensively studied on flat surfaces, their use on micro- and nanoparticles remains largely unexplored, despite the widespread experimental uncertainties that arise due to biofouling. Here we describe the preparation of magnetic micron-sized beads coated with zwitterionic sulfobetaine polymer brushes that display strong antifouling characteristics. These coated beads can then be equipped with recognition elements of choice, to enable the specific binding of target molecules. Firstly we present a proof of principle with biotin-functionalized beads that are able to specifically bind fluorescently labeled streptavidin from a complex mixture of serum proteins. Moreover, we show the versatility of the method by demonstrating that it is also possible to functionalize the beads with mannose moieties to specifically bind the carbohydrate-binding protein concanavalin A. Flow cytometry is used as a suitable read-out system, as this shows via a quick and automated analysis of thousands of individual beads per second, that thus modified beads only bind the specifically targeted proteins, with minimal non-specific protein adsorption from other proteins that are present. These antifouling zwitterionic polymer-coated beads therefore provide a significant advancement for the many diagnostic and other bio-sensing applications that require stringent antifouling conditions.
\end{abstract}




\subsection{Introduction}

Nano- and micron-sized particles have emerged as powerful platforms for many biomedical applications, ${ }^{1-5}$ including imaging, drug delivery, cell sorting as well as for biomolecule detection, separation and quantification. Especially magnetic particles have been shown to be highly valuable due to their quick and easy separation from their sample matrix, simply by applying an external magnetic field. ${ }^{1-2}$ Despite their frequent use in biomedical applications, these particles often suffer from the non-specific adsorption of biomolecules like cells and proteins, a process called biofouling. ${ }^{5-7}$ In sensing applications, fouling of the particle surface hampers the sensitivity and selectivity towards its target, leading to deteriorated signal-to-noise ratios and resulting in false positive or false negative outcomes..$^{8-9}$ To improve the performance of these particles, it is crucial to incorporate antifouling coatings on the surface of the particles to prevent biofouling.

Antifouling coatings have been extensively studied, yet mainly on flat surfaces. ${ }^{10-11}$ Oligo(ethylene glycol) (OEG) and poly(ethylene glycol) (PEG)-based materials are the most studied and frequently used antifouling coatings. ${ }^{12,13}$ However, PEG-based materials are prone to autoxidation, which limits their use for applications requiring long-term stability. ${ }^{14-15}$ Moreover, their performance is limited when exposed to complex, real-life biological fluids, ${ }^{16}$ i.e. the conditions typically observed in biomedical applications. Polymeric zwitterionic materials have been brought forward as excellent alternatives due to their high protein resistance in complex protein mixtures such as blood plasma and serum, ${ }^{16-17}$ combined with their long-term stability in aqueous solution, ${ }^{18}$ and biocompatibility. ${ }^{19}$ Grafting-from polymerization via Atom Transfer Radical Polymerization (ATRP), ${ }^{20}$ i.e. growing polymer brushes from a surface, has become the method of choice for the preparation of zwitterionic coatings. It yields antifouling, densely packed coatings with tunable thicknesses. ${ }^{21}$ Carboxybetaines (CB) and sulfobetaines (SB) have become the zwitterionic monomers of choice due to their commercial availability, straightforward synthesis and outstanding performance. ${ }^{22-23}$

Although antifouling coatings have been widely investigated on flat surfaces, their use in nano- and micro-sized particles remains largely unexplored. Apart from a small number of studies describing the use of PEG-derived materials, ${ }^{9,}{ }^{24}$ or zwitterionic coatings prepared by a grafting-to method with modified poly(acrylic acid), ${ }^{8,25}$ only two preliminary studies by Jia et al. ${ }^{6}$ and Yang et al. ${ }^{7}$ describe ATRP-prepared zwitterionic carboxybetaine polymer (pCB) coated nanoparticles for sensing purposes. However, both of these studies lack not only a sophisticated biological read-out system that allows for the discrimination between specific and non-specific protein binding, but also the ability to perform analysis on single beads rather than on the bulk. We anticipated therefore, that a sensing platform based on ATRP-prepared antifouling beads that can be analyzed by flow cytometry would be highly valuable. Flow cytometry is indispensable in biomedical research and is routinely used in clinical diagnostics, e.g. to monitor the course and treatment of HIV infections and to determine the prognoses and optimal treatment for several types of cancers. ${ }^{26-28}$ Moreover, flow cytometry allows for the simultaneous and multiparametric analysis of hundreds to thousands of cells or micron-sized 
particles per second, ${ }^{26}$ which makes it a very powerful and frequently used read-out system. Here we demonstrate that flow cytometry is also an excellent technique to study specific versus non-specific binding on micron-sized particles.

A

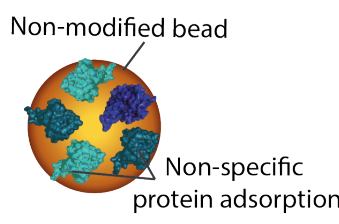

B

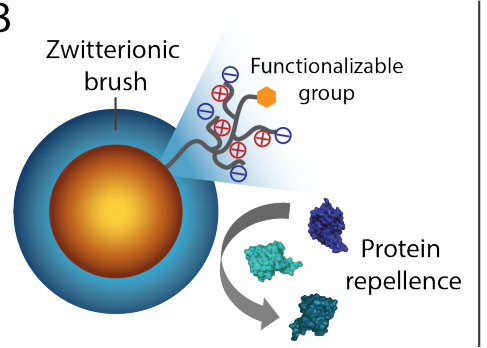

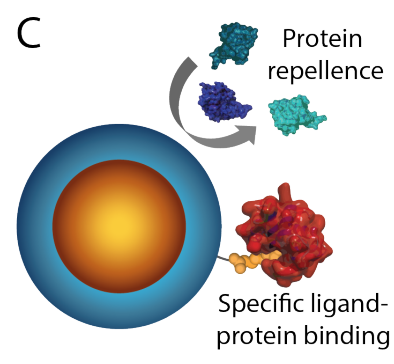

Figure 3.1 Schematic representation of (A) a non-modified bead with a significant amount of non-specifically bound proteins, (B) an antifouling zwitterionic polymer-coated bead that repels all biomolecules (before functionalization with a biorecognition unit), and (C) an antifouling polymercoated bead equipped with a recognition unit that specifically binds its target while still being able to repel all non-wanted proteins.

In this chapter we describe a route towards generically antifouling zwitterionic sulfobetaine (pSB)-coated beads that still can bind a specific protein of interest (see Figure 3.1). To this aim, magnetic amine-functionalized beads (Dynabeads; $2.8 \mu \mathrm{m}$ diameter) were selected as starting material. These beads are compatible with flow cytometry instruments, stable in both aqueous solutions and organic solvents, and can be easily separated from solvents/reactants due to their magnetic core. Top-functionalization of the pSB-coated beads with biotin or mannose allowed for the specific binding of streptavidin or concanavalin A (ConA) from complex biological media, respectively. These pSB-coated beads showed excellent antifouling properties combined with the selective binding of the protein of interest. This platform shows therefore great potential for the development of a range of bio-assays that require ultra-low biofouling conditions.

\subsection{Materials and methods}

\subsubsection{Materials}

All chemicals and solvents were used without further purification. $\alpha$-Bromoisobutyrylbromide (98\%), trimethylamine (BioUltra, $\geq 99.5 \%$ ), copper (I) chloride ( $\geq 99 \%)$, copper(II) chloride (97\%), sodium azide (BioUltra, $\geq 99.5 \%$ ), DMSO (anhydrous, $\geq 99.9 \%$ ), acetone semiconductor grade (VLSI PURANAL ${ }^{\mathrm{TM}}$ Honeywell 17617), tris(3-hydroxypropyltriazolylmethyl) amine (THPTA; 95\%), copper(II) sulfate pentahydrate (98\%) and (+)-sodium L-ascorbate 
(BioXtra, $\geq 99.0 \%$ ) were purchased from Sigma-Aldrich. Dimethylformamide (DMF) for peptide synthesis (99.8\%) and mono-propargylamine ( $\geq 99 \%)$ were obtained from Acros Organics, 2,2'-bipyridine (98\%) from Alfa Aesar, isopropanol (HPLC) from Biosolve, 2,2,3,3,4,4,4-heptafluorobutylamine (97\%) from Fisher Scientific, dichloromethane (DCM) from VWR International S.A.S., and 8-(+)-biotinylamino-3,6-dioxa-octyl(1R,8S,9S)bicyclo[6.1.0]non-4-yn-9-ylmethyl carbamate (BCN-biotin) from SynAffix. MilliQ was produced with a MilliQ Integral 3 system (Millipore).

Dynabeads (Dynabeads ${ }^{\mathrm{TM}} \mathrm{M}-270$ amine; $2.8 \mu \mathrm{m}$ diameter) and concanavalin A-Alexa Fluor 647 conjugate (ConA-AF647) were purchased from Invitrogen Life Technologies. Bovine serum albumin-Alexa Fluor 488 conjugate (BSA-AF488) was obtained from Thermo Fisher and streptavidin-phycoerythrin conjugate (Strep-PE) from eBioscience. Bovine blood sample collection was approved by the Board on Animal Ethics and Experiments from Wageningen University (DEC number: 2014005.b).

\subsubsection{Synthesis}

The synthesis of all non-commercially available compounds has been previously described, as detailed below. The 3-((3-methacrylamidopropyl)dimethylammonio)propane-1-sulfonate (SB) monomer and $\mathrm{BCN}-2,2,2$,-trifluoroacetate $\left(\mathrm{BCN}_{-} \mathrm{CF}_{3}\right)$ were described by Lange et al., ${ }^{29}$ 1 -(11-azidoundecanyl)- $\alpha$-D-mannopyranoside (mannose- $\mathrm{C}_{11}$-azide) by Debrassi et al., ${ }^{30}$ and the $13,13,14,14,15,15,16,16,16$-nonafluorohexadec-1-yne ( $\mathrm{F}_{9}$-alkyne) by Pujari et al. ${ }^{31}$

\subsubsection{Bead handling}

During all collection and washing steps, beads were collected using a magnetic stand (Promega) which allows for quick and easy separation of the beads from solvent and reactants. All reactions described below are based on $100 \mu \mathrm{L}$ (corresponding to approximately $3 \mathrm{mg}$ beads, containing $\sim 2 \times 10^{8}$ beads) of the original non-modified bead suspension as provided by the manufacturer. If no bead volume is mentioned, solvent (MilliQ) was removed and the beads were resuspended in the solvent of choice. In all cases, unless stated otherwise, $2 \mathrm{~mL}$ Eppendorf tubes were used for bead modifications. After each reaction, the beads were stored in a small amount of MilliQ at $4{ }^{\circ} \mathrm{C}$ until further use.

\subsubsection{Initiator attachment}

The bromo-initiator was incorporated by first drying $100 \mu \mathrm{L}$ of amine-terminated Dynabeads in a vacuum oven at $50{ }^{\circ} \mathrm{C}$ for $2 \mathrm{~h}$. The dried beads were resuspended in $1.25 \mathrm{~mL}$ of dry DCM and transferred to a glass tube with screw-cap connection. The bead suspension was bubbled with argon while $700 \mu \mathrm{L}$ of triethylamine and $370 \mu \mathrm{L}$ (3 mmol) of $\alpha$-bromoisobutyryl bromide was added. The glass tube was closed with a screw cap, covered with aluminum foil and placed 
for $3 \mathrm{~h}$ on an end-over-end shaker at RT. The beads were washed with copious amounts of DCM to dissolve any precipitate that might have formed. The beads were transferred to an Eppendorf tube and washed twice with isopropanol and twice with MilliQ.

\subsubsection{Surface-initiated polymerization}

Surface-initiated Atom Transfer Radical Polymerization (SI-ATRP) was performed as previously described $^{29}$ with slight modifications. Solvents were degassed by sonication (5 min) and argon bubbling for 30 min. Reactants were kept under argon atmosphere during all steps. A mixture of $41.0 \mathrm{mg}$ 2,2-bipyridine and $12.1 \mathrm{mg}$ of a $\mathrm{Cu}(\mathrm{I}) \mathrm{Cl} / \mathrm{Cu}(\mathrm{II}) \mathrm{Cl}_{2}(9 / 1)$ mixture was prepared in a glove box under an argon atmosphere, dissolved in $5.25 \mathrm{~mL}$ of isopropanol/ MilliQ (1/4) and stirred for $15 \mathrm{~min}$. Using argon flushed needles, $2 \mathrm{~mL}$ of the resulting brown solution (containing $0.1 \mathrm{mmol}$ (1 eq.) bipyridine and $0.045 \mathrm{mmol}$ (0.45 eq) copper mix) was then transferred to a Schlenck flask containing $731 \mathrm{mg}(2.5 \mathrm{mmol}, 25$ eq.) of the 3-((3-methacrylamidopropyl)dimethylammonio)propane-1-sulfonate (SB) monomer. This mixture was stirred for $15 \mathrm{~min}$, or until full solubilization of the SB monomer. Meanwhile, the required amount of initiator-functionalized beads was bubbled with argon for $10 \mathrm{~min}$ in a total volume of $1 \mathrm{~mL}$ of degassed isopropanol/MilliQ (1/4). The monomer-containing solution was then transferred $(2 \mathrm{~mL})$ to the beads to a final volume of $3 \mathrm{~mL}$. The polymerization reaction was carried out for $1 \mathrm{~min}$ at RT, while shaking by hand to ensure proper dispersion of the beads. The reaction was stopped by pouring the solution into an Erlenmeyer flask and adding MilliQ while swirling, until the solution turned blue. The blue color indicates the oxidation, and thereby inactivation, of the copper catalyst, which hence stops the polymerization reaction. The pSB-coated beads were collected using a magnet and washed two times with isopropanol/ MilliQ (1/4), twice with PBS, and twice with MilliQ.

\subsubsection{Top-functionalization of polymer-coated beads}

\section{FLUOROBUTYLAMINE ${ }^{32}$}

Substitution of the terminal halogen with a fluorine-containing molecule for XPS analysis was achieved byimmersing $p$ SB-coated beadsina 2Msolution of 2, 2, 3, 3, 4,4,4-heptafluorobutylamine in DMF $(290 \mu \mathrm{L}$ of the fluoroamine and $210 \mu \mathrm{L}$ of DMF), in a small glass tube closed with a screwcap. The glass tube was covered with aluminum foil and placed in an oil bath at $65{ }^{\circ} \mathrm{C}$; the beads were allowed to react for $16 \mathrm{~h}$. The next day, the reaction tube was cooled to RT and the beads were transferred to an Eppendorf tube and washed twice with DMF, twice with PBS and twice with MilliQ.

\section{AZIDE INTRODUCTION 33}

Azide moieties were introduced on pSB-coated beads by immersing the beads in $1 \mathrm{~mL}$ of a 0.5 $\mathrm{M}$ solution of sodium azide in PBS in a with aluminum foil covered Eppendorf tube. The tube 
was placed on an end-over-end shaker for $16 \mathrm{~h}$ at RT. The pSB-azide beads were 3 times washed with PBS and twice with MilliQ.

\begin{abstract}
ALKYNE INTRODUCTION
Alkynes were introduced using a $2 \mathrm{M}$ solution of propargylamine $(128 \mu \mathrm{L}$ for $1 \mathrm{~mL})$ in PBS. The solution was added to the pSB beads, which were allowed to react in an Eppendorf tube, covered with aluminum foil, overnight on an end-over-end shaker at RT. The pSB-alkyne beads were 3 times washed with PBS and twice with MilliQ.
\end{abstract}

\title{
3.2.7 Click chemistry: brush functionalization
}

\section{BRUSH FUNCTIONALIZATION USING STRAIN-PROMOTED AZIDE-ALKYNE CYCLOADDITION (SPAAC) ${ }^{29}$}

For the reaction of azide-functionalized polymer brush-coated beads with BCN-biotin or BCN$\mathrm{CF}_{3}$, first a $40 \mathrm{mM}$ stock solution of the $\mathrm{BCN}$ compound was prepared in anhydrous DMSO (stored at $-20{ }^{\circ} \mathrm{C}$ ). To an Eppendorf tube, $100 \mu \mathrm{L}$ of this solution and $100 \mu \mathrm{L}$ of pSB-azide beads, suspended in MilliQ, were added to a final BCN concentration of $20 \mathrm{mM}$. The tube was placed on an end-over-end shaker overnight at RT; the beads were then subsequently washed two times with DMSO, twice with PBS and twice with MilliQ.

\section{BRUSH FUNCTIONALIZATION USING COPPER-CATALYZED AZIDE-ALKYNE CYCLOADDITION (CUAAC)}

For the click reaction on azide-containing beads with 13,13,14,14,15,15,16,16,16nonafluorohexadec-1-yne ( $\mathrm{F}_{9}$-alkyne), two stock solutions were prepared: a $10 \mathrm{~mL}$ aqueous solution containing $2.5 \mathrm{mM} \mathrm{CuSO}_{4} \cdot 5 \mathrm{H}_{2} \mathrm{O}(6.24 \mathrm{mg})$ and $50 \mathrm{mM}(+)$-sodium L-ascorbate $(99.0$ $\mathrm{mg}$ ), and a $1 \mathrm{~mL}$ solution of $12.5 \mathrm{mM}$ tris(3-hydroxypropyltriazolylmethyl)amine (THPTA, $5.43 \mathrm{mg}$ ) and $100 \mathrm{mM}$ of the $\mathrm{F}_{9}$-alkyne $(38.4 \mathrm{mg}$ ) in DMSO. $100 \mu \mathrm{L}$ of each solution was added to an Eppendorf tube and 10 times diluted by adding the alkyne/azide-functionalized beads suspended in $800 \mu \mathrm{L}$ of DMSO/MilliQ (1/1). This resulted in the following final concentrations: $0.25 \mathrm{mM} \mathrm{CuSO}_{4}, 5 \mathrm{mM}$ sodium ascorbate, $1.25 \mathrm{mMTHPTA}$ and $10 \mathrm{mM}$ of the alkyne. For an effective click reaction of mannose- $\mathrm{C}_{11}$-azide with alkyne-terminated beads, the protocol had to be modified, which was done via the guidelines provided by Hong et al. ${ }^{34}$ Three stock solutions were prepared: a $10 \mathrm{~mL}$ aqueous PBS solution containing $1 \mathrm{mM} \mathrm{CuSO} \cdot 5 \mathrm{H}_{2} \mathrm{O}$ (2.49 mg) and $5 \mathrm{mM}$ THPTA (21.7 mg), a $10 \mathrm{~mL}$ aqueous PBS solution containing $12.5 \mathrm{mM}$ (+)-sodium L-ascorbate $\left(24.8 \mathrm{mg}\right.$ ), and $2 \mathrm{~mL}$ containing $5 \mathrm{mM}$ mannose- $\mathrm{C}_{11}$-azide $(3.8 \mathrm{mg}$ ). The following was added, in this order, to an Eppendorf tube: $400 \mu \mathrm{L}$ of DMSO, $200 \mu \mathrm{L}$ of PBS containing the alkyne-terminated beads, $100 \mu \mathrm{L}$ of the $\mathrm{CuSO}_{4} / \mathrm{THPTA}$ solution, $100 \mu \mathrm{L}$ of the mannose- $\mathrm{C}_{11}$-azide solution and finally $200 \mu \mathrm{L}$ of the sodium ascorbate solution to initiate the reaction. The final concentrations of the reactants: $100 \mu \mathrm{M} \mathrm{CuSO}_{4}$, $500 \mu \mathrm{M}$ THPTA, $2.5 \mathrm{mM}$ ascorbate and $500 \mu \mathrm{M}$ of mannose- $\mathrm{C}_{11}$-azide. 
The CuAAC reactions were performed overnight (16 h) on an end-over-end shaker at RT. The beads were washed twice with DMSO, DMSO/MilliQ (1/1), PBS and MilliQ, respectively.

\subsubsection{Bead characterization}

\section{XPS}

X-ray Photoelectron Spectroscopy (XPS) samples were prepared by concentrating the beads (in MilliQ) and dropcasting $10 \mu \mathrm{L}$ of this suspension onto a piece of $\mathrm{Si}(111)$ (Siltronix, N-type, phosphorus doped), which was cleaned by rinsing and sonicating for $5 \mathrm{~min}$ in semiconductor grade acetone followed by oxygen plasma treatment (Diener electronic, Femto A) for 5 min at $50 \%$ power. The dropcast samples were subsequently dried in a vacuum oven at $50{ }^{\circ} \mathrm{C}$ for at least $2 \mathrm{~h}$ before XPS measurements were started. XPS spectra were obtained using a JPS-9200 photoelectron spectrometer (JEOL) with monochromatic Al-K $\alpha$ X-Ray radiation at $12 \mathrm{kV}$ and $20 \mathrm{~mA}$. The obtained spectra were analyzed using CASA XPS software (version 2.3.16 PR 1.6).

\section{FT-IR}

Fourier Transform Infrared Spectroscopy (FT-IR) samples were prepared by drying $\sim 10 \mu \mathrm{L}$ of MilliQ-washed beads in a vacuum oven at $50{ }^{\circ} \mathrm{C}$ for at least $2 \mathrm{~h}$. The dried beads were transferred to a small piece of gold-coated $\mathrm{Si}(111)$ and subsequently measured by a Bruker Tensor 27 IR spectrometer, connected to a Bruker Hyperion 2000 IR microscope with a liquid nitrogen-cooled MCT-detector. Both apparatuses were controlled using Bruker's OPUS software. The microscope was used to select an area with a sufficient amount of beads and a proper background position. 128 Scans were taken for each background and sample measurement.

\section{FLOW CYTOMETRY}

Beads were suspended in $0.5 \mathrm{~mL}$ of PBS and shortly vortexed before measuring with a BD FACS Canto II ${ }^{\mathrm{TM}}$ (BD Biosciences) flow cytometer. Per sample 10.000 single beads were measured; for the gating strategy that was used, see Supporting Information. Data analysis was performed using FlowJo software V10.

\section{CONFOCAL MICROSCOPY}

Fluorescence images were taken using a confocal laser scanning microscope (Leica TCS SP8X system), equipped with a $63 \times / 1.2 \mathrm{NA}$ water immersion objective. BSA-AF488 and Strep-PE were excited using a white light pulsed (repetition rate $40 \mathrm{MHz}$ ) laser selecting the 488-laser line and 561-laser line, respectively. Fluorescence emission was collected between 500-530 $\mathrm{nm}$ for AF488 and the PE fluorescence emission was selected by measuring between 570-610 $\mathrm{nm}$. Images were captured in photon-counting mode, accumulating photons over 10 frames. The pinhole was set to 0.39 Airy units, and an optical zoom of 8.00 was used to capture the images. Confocal images were analyzed with the LAS AF Lite (version 2.6.0) software. 


\subsubsection{Antifouling and specific binding studies}

\section{SERUM LABELING}

Bovine blood serum samples were obtained from healthy cows via coccygeal vein sampling. Serum was collected using VACUETTE® tubes (4 mL Z Serum Separator Clot Activator $13 \times$ 75 gold cap-gold ring, PREMIUM) from Greiner Bio-One. Serum from 3 individual cows was pooled and heated for $30 \mathrm{~min}$ at $56{ }^{\circ} \mathrm{C}$ in a water bath (to inactivate complement) and stored at $-20{ }^{\circ} \mathrm{C}$. Labeling of serum proteins was performed using an AnaTag ${ }^{\mathrm{TM}}$ HiLyte $^{\mathrm{TM}}$ Fluor 488 microscale protein labeling kit (AnaSpec, Inc.) according to the manufacturer's instructions using $84 \mu \mathrm{L}$ of serum per batch. After labeling, the samples were concentrated using Amicon Ultra-4 Centrifugal Filter tubes (Millipore BV). The filters were first washed with PBS, labeled serum was added and the tubes were centrifuged for $35 \mathrm{~min}$ at $3363 \mathrm{~g}$ at $4{ }^{\circ} \mathrm{C}$. The obtained concentrated labeled serum had a final concentration of $21.2 \mathrm{mg} / \mathrm{mL}$. From this a $10 \%$ SerumHLF488 solution was reconstituted with a concentration of $6 \mathrm{mg} / \mathrm{mL}$.

\section{SERUM BIOTINYLATION}

The same pooled cow's serum that was used for the HiLyte Fluor 488 serum labeling was also used for biotinylation. Serum proteins were biotinylated using an EZ-Link Sulfo-NHSLC-Biotin reagent, using the manufacturer's instructions. To a $2 \mathrm{~mL}$ Eppendorf tube, $60 \mathrm{mg}$ of serum proteins and $429 \mu \mathrm{L}$ of a $100 \mathrm{mM}$ sulfo-NHS-biotin solution (in PBS) were added to a final volume of $2 \mathrm{~mL}$. Assuming that all proteins in serum have a molecular weight of $70 \mathrm{kDa}$, 50 equivalents of sulfo-NHS-biotin was used relative to the amount of proteins. The reaction was carried out at RT for $60 \mathrm{~min}$. Non-bound reagents were removed by using a desalting PD-10 column (Sephadex), following the manufacturer's gravitation protocol with PBS as the eluent. The concentration of the obtained biotinylated serum (Serum-Biotin) was adjusted to a $10 \%$ serum solution $(6 \mathrm{mg} / \mathrm{mL})$.

\section{PROTEIN DILUTION SERIES}

Two-fold serial dilutions ( $200 \mu \mathrm{L}$ each) were prepared from the following protein solutions: $1 \mathrm{mg} / \mathrm{mL}$ bovine serum albumin-Alexa Fluor 488 conjugate (BSA-AF488), $100 \mu \mathrm{g} / \mathrm{mL}$ streptavidin-phycoerythrin conjugate (Strep-PE) and 10\% HiLyte Fluor 488-labeled serum (Serum-HLF488, $\sim 6 \mathrm{mg} / \mathrm{mL}$ ). To each dilution, either $2 \times 10^{6}$ non-modified beads or $2 \times 10^{6}$ pSB beads were added. Beads were counted using a cell-counting chamber to ensure equal amounts of beads. Samples were covered with aluminum foil and placed on an end-over-end shaker for $1 \mathrm{~h}$ at RT. The beads were washed 3 times with PBS and analyzed by flow cytometry for their fluorescence.

\section{QUANTIFICATION BY ELISA}

Non-modified beads and $p$ SB-coated beads $\left(10 \times 10^{6}\right.$ beads per sample, counted using cell 
counting chamber) were incubated with Serum-Biotin, washed 3 times with PBS, followed by incubation with horseradish peroxidase conjugated streptavidin (Strep-HRP. 1:100 dilution from purchased stock solution) or directly with Strep-HP. Beads were washed five times with PBS, transferred to a new Eppendorf tube, and washed an additional 5 times to ensure that all non-bound Strep-HRP had been removed. Twofold serial dilutions (1.7 serial dilutions for the Serum-Biotin samples) were made of the bead samples, with each a final volume of $50 \mu \mathrm{L}$. To each dilution, $200 \mu \mathrm{L}$ 3,3',5,5'-tetramethylbenzidine (TMB) solution was added, followed by $100 \mu \mathrm{L} \mathrm{4 \%} \mathrm{HCl}$ to stop the enzymatic reaction. The optical density was measured in a 96well plate at $450 \mathrm{~nm}$ and subtracted by the absorbance measured at $620 \mathrm{~nm}$ to correct for irregularities within the plastic.

\section{PROTEIN BINDING STUDIES}

For each sample, $100 \mu \mathrm{L}$ of protein solution (or PBS only) was prepared into $2 \mathrm{~mL}$ Eppendorf tubes prior to the addition of $2 \mu \mathrm{L}$ of beads. Single protein solutions or protein mixtures were prepared in the following concentrations: $50 \mu \mathrm{g} / \mathrm{mL}$ Strep-PE, $50 \mu \mathrm{g} / \mathrm{mL}$ concanavalin A-Alexa Fluor 647 conjugate (ConA-AF647), $0.5 \mathrm{mg} / \mathrm{mL}$ BSA-AF488, and 10\% Serum-HLF488( 6 $\mathrm{mg} / \mathrm{mL})$. PBS buffer with additional $\mathrm{CaCl}_{2}(1 \mathrm{mM})$ was used for each sample that contained ConA-AF647. After adding the coated beads to the protein solutions, the tubes were covered with aluminum foil and placed on an end-over-end shaker for $1 \mathrm{~h}$ at RT. The beads were then washed 3 times with PBS and analyzed by either flow cytometry or confocal microscopy.

\subsection{Results and discussion}

\subsubsection{Coating beads with zwitterionic polymer brushes}

Our first step in the surface modification towards an antifouling bead with installed recognition elements was the introduction of an Atom Transfer Radical Polymerization (ATRP) initiator, which could be achieved straightforwardly by reacting the amino groups of the beads with the acid bromide group of bromoisobutyryl bromide (Scheme 3.1, route a). Successful incorporation of the initiator was confirmed by X-ray Photoelectron Spectroscopy (XPS) analysis (Figure 3.2), which showed the appearance of distinctive peaks characteristic for the element bromide (see Supporting Information, Figure S3.2 for XPS narrow scans). Moreover, FT-IR spectroscopy demonstrated the appearance of a carbonyl peak at $1732 \mathrm{~cm}^{-1}$ (see Supporting Information, Figure S3.9). From the installed initiator, zwitterionic polymer brushes were grown via ATRP, ${ }^{20}$ based on our previously described procedure. ${ }^{29}$ Polymer brushes were grown using the zwitterionic sulfobetaine monomer (SB) to yield pSB-coated beads (Scheme 3.1, route b).

The beads were analyzed by XPS (Figure 3.2), and the growth of the zwitterionic coating was confirmed by the appearance of two sulfur peaks, S 2s $(232.0 \mathrm{eV})$ and S 2p $(168.0 \mathrm{eV})$, originating from the negatively charged sulfonate group. In addition, the XPS N 1s spectrum 

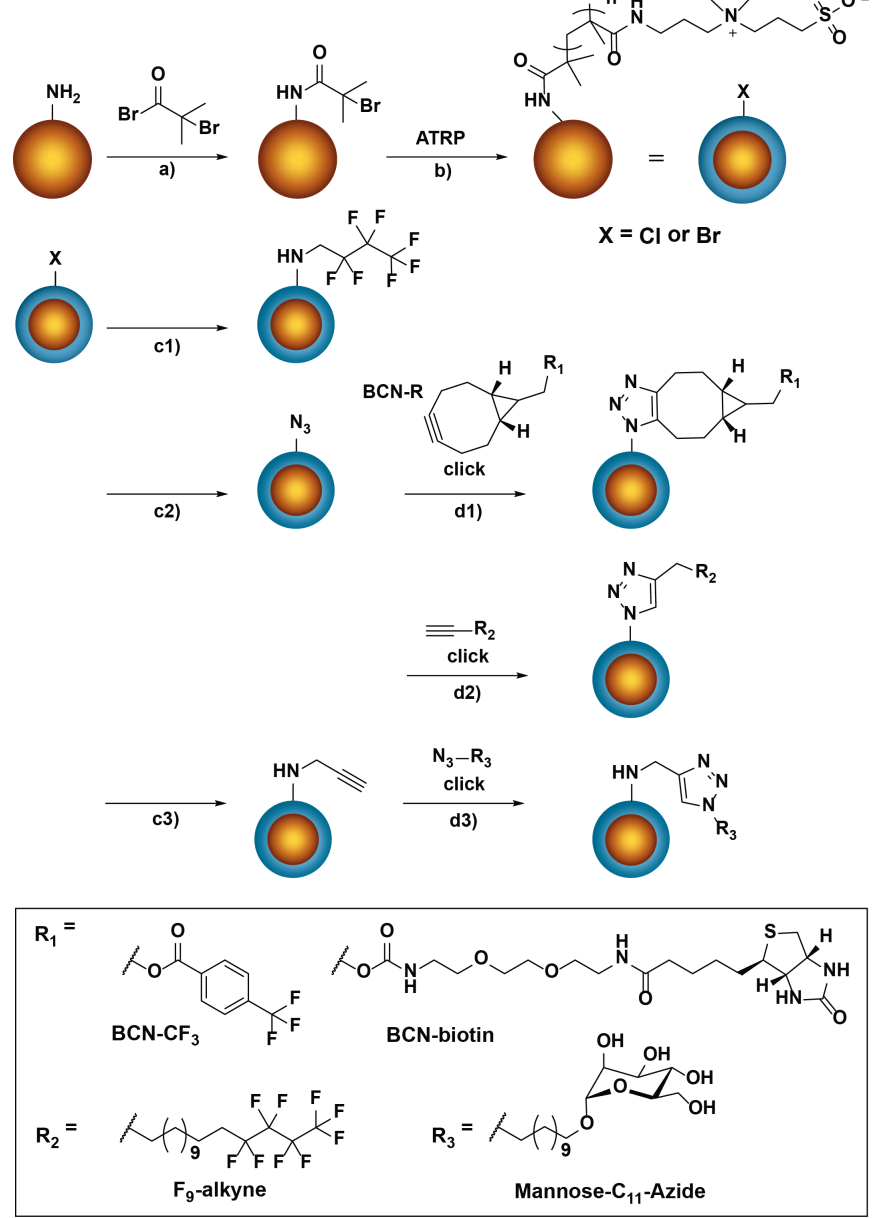

Scheme 3.1 Overview of chemical modifications on amine-terminated beads to yield functionalized antifouling pSB-coated beads. The reaction conditions represented by the arrows are: (a) $2.4 \mathrm{M}$ 2-bromoisobutyryl bromide, DCM, Et ${ }_{3} \mathrm{~N}, \mathrm{RT}, 3 \mathrm{~h}$. (b) $\mathrm{Cu}(\mathrm{I}) / \mathrm{Cu}(\mathrm{II})(9 / 1)$, bipyridine, isopropanol/ MilliQ (1/4), $1 \mathrm{mmol} \mathrm{SB}, \mathrm{RT}, 1 \mathrm{~min}$. (c1) $2 \mathrm{M}$ heptafluorobutylamine, DMF, $65{ }^{\circ} \mathrm{C}, 16 \mathrm{~h}$. (c2) $0.5 \mathrm{M}$ $\mathrm{NaN}_{3}$, PBS, RT, 16 h. (c3) 2 M propargylamine, PBS, RT, 16 h. (d1) SPAAC, 20 mM BCN-R , DMSO/MilliQ (1/1), RT, 16 h. (d2) CuAAC, $10 \mathrm{mM} \mathrm{F}_{9}$-alkyne, $0.25 \mathrm{mM} \mathrm{CuSO}_{4}, 5 \mathrm{mM}$ sodium ascorbate, $1.25 \mathrm{mM}$ THPTA, DMSO/MilliQ (1/1), RT, 16 h. (d3) CuAAC, $500 \mu \mathrm{M}$ mannose-C ${ }_{11}$-azide, $100 \mu \mathrm{M} \mathrm{CuSO}_{4}, 2.5$ mM sodium ascorbate, $500 \mu \mathrm{M}$ THPTA, DMSO/MilliQ (1/1), RT, 16 h..

shows the expected characteristic 1: 1 ratio of the amide (409.5 eV) and ammonium (412.4 $\mathrm{eV})$ peaks. The equal ratio of these two peaks implies that the signal of the underlying bead can no longer be detected, which indicates a sufficiently thick polymer layer (clearly in excess of 
$\sim 10 \mathrm{~nm}$ ) that is required for good antifouling performance. ${ }^{18}$ The XPS C 1 s spectrum displays three distinguishable peaks corresponding to the $\mathrm{C}-\mathrm{C}$ carbon atoms at $285.0 \mathrm{eV}$, the carbon atoms next to a heteroatom $(\mathrm{C}-\mathrm{O}, \mathrm{C}-\mathrm{N}$ and $\mathrm{C}-\mathrm{S})$ at $286.3 \mathrm{eV}$ and a peak corresponding to the carbonyl of the amide bond at $287.7 \mathrm{eV}$. Reassuringly, the XPS wide scan, N 1s and C 1s spectra of the polymer brush-coated beads are practically identical to previously obtained spectra for flat surfaces (see chapter 2). ${ }^{18,29}$ Worthwhile to mention is the small, remaining Br peak in the
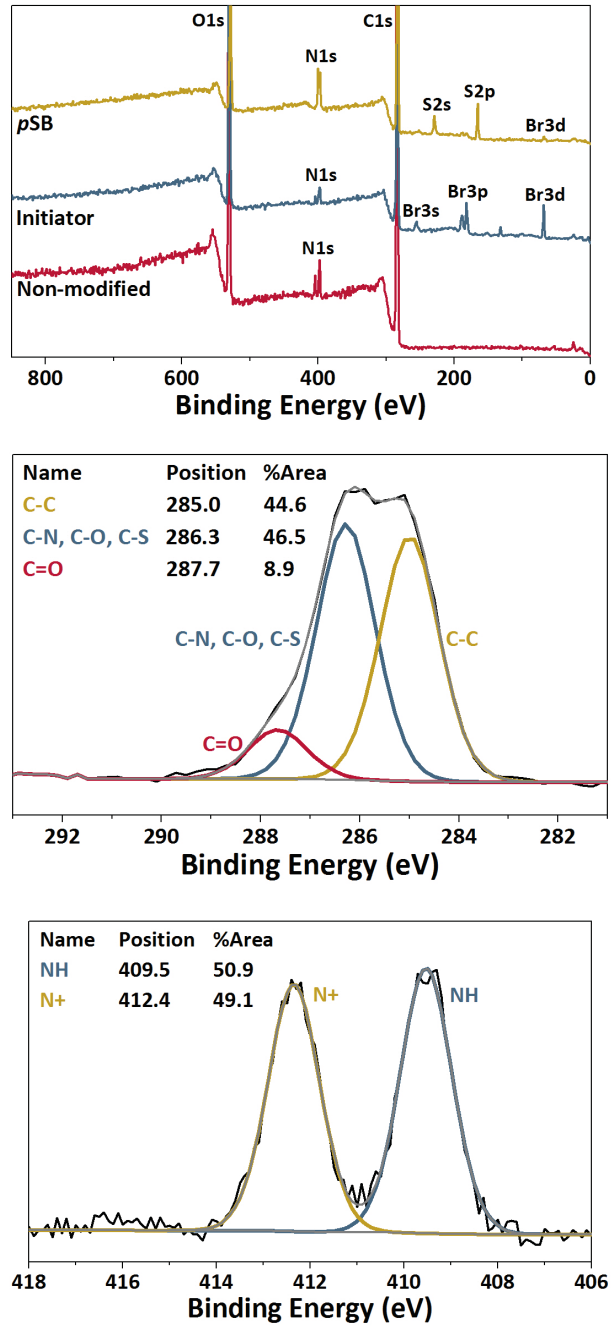

Figure 3.2 (A) XPS wide scans of non-modified beads, initiator-functionalized and pSB polymer-coated beads. (B) XPS C 1s and (C) N 1s narrow scans of pSB-coated beads. The spectra show the successful growth of zwitterionic polymer brushes from the beads. 
XPS wide scan of the pSB-coated beads that can still be discerned in the top spectrum of Figure 3.2A. This peak can be attributed to the terminal bromides that are retained at the polymer chain ends after ATRP. As described below, these bromides offer a useful functional handle to introduce specific recognition elements.

\subsubsection{Antifouling properties of pSB-coated beads}

Traditionally, antifouling properties of coated surfaces are evaluated by investigating the amount of non-specifically bound proteins by using single-protein solutions of bovine serum albumin (BSA), lysozyme or fibrinogen. ${ }^{12-13,22}$ More recently, it has become common practice to also include more complex biological media such as (diluted) blood plasma or serum. These more challenging conditions are typically used in biomedical applications and also turned out to be required to discriminate a high-efficacy antifouling surface from a moderately protein-repelling one. ${ }^{35,36}$ For the evaluation of our pSB-coated beads, we therefore selected not only fluorescently labeled BSA (BSA-AF488, $0.5 \mathrm{mg} / \mathrm{mL}$ ), but also included an in-house fluorescently labeled 10\% cow's serum solution (Serum-HLF488, 6 mg/mL). The SerumHLF488 solution has a 12 times higher total protein concentration than the BSA-AF488 solution, and contains - aside from BSA - also naturally occurring immunoglobulins and other serum proteins. Immunoglobulins, among other proteins like human serum albumin (HSA) and complement components, have been found on PEG-based surfaces that were exposed to human blood plasma, ${ }^{37}$ and these types of proteins might therefore contribute to fouling of the beads presented in this study. The ability of the pSB-coated beads to repel all the proteins in the used BSA and serum solutions was evaluated using flow cytometry. To this end, nonmodified beads and pSB-coated beads were incubated with either the BSA-AF488 or SerumHLF488 solution, and their fluorescence was measured after several washing steps. Per sample, 10,000 single beads were analyzed (see Supporting Information, Figure S3.10, for the gating strategy and corresponding explanation on gating). Figure 3.3 shows the fluorescence data of these beads, displayed as a histogram, showing the number of beads for a given fluorescent intensity of a selected fluorophore. Beads incubated in protein-free PBS are taken as reference point, while beads with a higher fluorescence than the PBS beads (shifted to the right side of the gate, which is depicted as a dashed line in the histogram) are considered as positive. Positive beads therefore indicate a significant increase in fluorescence, and therefore protein binding, as compared to beads incubated in PBS only. A pronounced shift in fluorescence was observed (Figure 3.3A) for non-modified beads that were incubated with either BSA-AF488 or Serum-HLF488, indicating a significant amount of non-specifically bound proteins on these beads. In other words, non-modified beads suffer strongly from protein fouling. The pSB-coated beads, however, showed no difference in fluorescence between incubation in PBS or BSAAF488 solution (as can be concluded from the identical blue and dark green peaks in Figure 3.3B), and only a minor increase when comparing the beads incubated with Serum-HLF488 to beads incubated in PBS. This clearly demonstrates the excellent antifouling properties of the pSB-coated beads: the coating strongly reduces non-specific binding of fluorescently labeled proteins, including proteins from a complex protein mixture. 

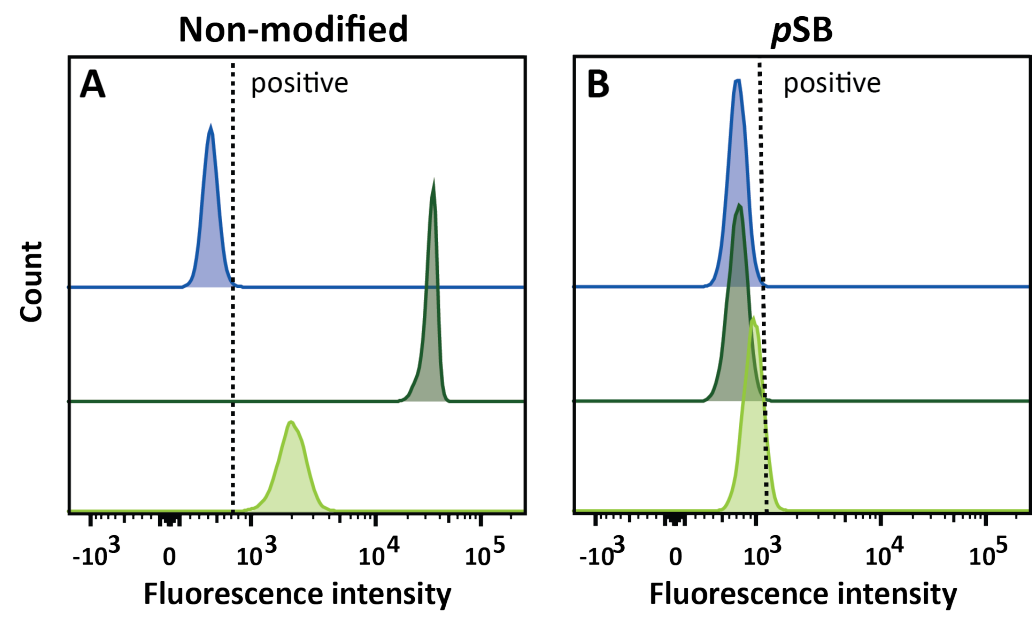

Figure 3.3 Schematic representation of (A) a non-modified bead with a significant amount of non-specifically bound proteins, (B) an antifouling zwitterionic polymer-coated bead that repels all biomolecules (before functionalization with a biorecognition unit), and (C) an antifouling polymercoated bead equipped with a recognition unit that specifically binds its target while still being able to repel all non-wanted proteins.

The mean fluorescence intensity (MFI), normalized to the intensity of beads incubated in PBS, of non-specifically bound proteins to either non-modified or pSB-coated beads is summarized in Figure 3.4. Also from these data it is evident that non-modified beads showed clear non-specific protein adsorption when exposed to BSA-AF488 and Serum-HLF488 solutions. Non-modified beads also showed a clear increase in the non-specific binding of fluorescently labeled ConA and a small but significant increase with labeled streptavidin (ConA-AF635 (MFI = 14053) and Strep-PE (MFI = 454), respectively), which are used for specific binding studies as described below. In contrast, pSB-coated beads displayed a nearly absent non-specific binding of BSAAF488, Strep-PE and ConA-AF635. Only under the most challenging biofouling conditions that were studied, i.e. incubation with Serum-HL488, pSB-coated beads started to show a slight increase in protein adsorption (MFI $=272$ ). However, this adsorption is only a fraction of the amount adsorbed on non-modified beads $(M F I=1830)$. Finally, it is worthwhile to point out that inherent to the used method, the MFI of different fluorescently labeled protein solutions cannot be directly compared with respect to the amount of bound protein (see Supporting Information).

To get further quantitative insight in the antifouling behavior of pSB-coated beads versus nonmodified beads, the beads were incubated with protein solutions at different concentrations. To this end, serial dilutions were prepared from BSA-AF488, Strep-PE, and serum-HLF488 solutions and added to the beads (see Supporting Information, Figure S3.12). In all cases, pSB- 


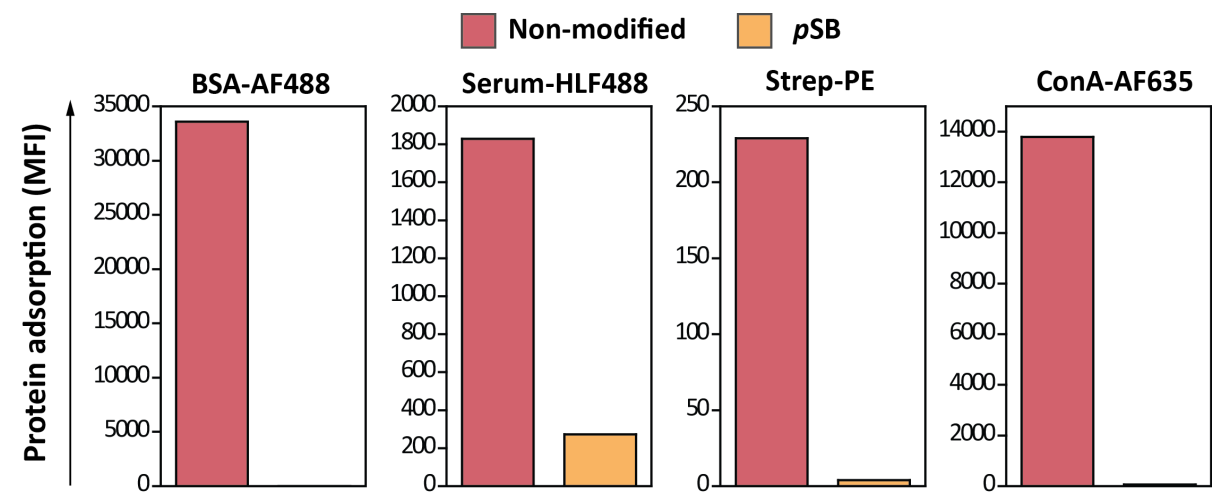

Figure 3.4 Representative flow cytometry data summarizing the mean fluorescence intensity (MFI) of non-modified and pSB-coated beads incubated with PBS, BSA-AF488 (0.5 mg/mL), Serum-HLF488 $(10 \%, \sim 6 \mathrm{mg} / \mathrm{mL})$, Strep-PE $(50 \mu \mathrm{g} / \mathrm{mL})$ or ConA-AF635 $(50 \mu \mathrm{g} / \mathrm{mL})$. MFI values are corrected for the auto-fluorescence of the beads by subtracting MFI values of their corresponding PBS samples, see Supporting Information, Table S3.1 for non-corrected values.

coated beads performed evidently better than non-modified beads. In fact, a direct comparison between non-modified beads and pSB-coated beads that were incubated with either BSAAF488 or Strep-PE was not feasible, as the amount of fouling on pSB beads was near zero. What can be concluded is that pSB-coated beads showed less fouling at the highest protein concentration than non-modified beads do with a more than 500 times diluted protein sample. Non-modified and pSB beads incubated with serum-HLF488 could be directly compared, as detectable fouling was also seen on pSB beads (for the four highest tested concentrations). Across these serum dilutions, ranging from 10 to $1.25 \%$, the pSB-coated beads showed a factor 6-10 less fouling (MFI) than non-modified beads. To verify these results, the amount of adsorbed protein on the beads was also assessed via a horseradish peroxidase (HRP) assay, similar to the well-known enzyme-linked immunosorbent assay (ELISA, see the Supporting Information, Figure S3.15). The beads were incubated with 10\% biotinylated serum followed by Strep-HRP incubation or Strep-HRP directly. Serial dilutions were prepared from the bead samples, and the enzymatic (by HRP) conversion of TMB was measured at $450 \mathrm{~nm}$. From the obtained titration curves the relative amounts of bound Strep-HRP could be determined, and hence the amount of bound biotinylated serum, by comparing the number of beads necessary to obtain half of the maximum optical density $\left(\mathrm{OD}_{450}\right.$ value). Almost no direct Strep-HRP adsorption could be detected - that is, in a solution in which Strep-HRP is the only protein, it basically does not absorb onto the pSB-coated beads - which makes a quantitative comparison with nonmodified beads unfeasible. Only when using serum, labeled with biotin and in the presence of Strep-HRP, fouling was seen on PSB beads, and thus a comparison could be made with nonmodified beads. Five to six times more pSB beads were needed to obtain an amount of signal similar to non-modified beads. This indicates that under these conditions, pSB beads contain 5-6 times less adsorbed serum proteins than the non-modified beads. The quantification by 
ELISA, therefore, corresponds well with the MFI ratio of 6 as obtained by flow cytometry for the same serum dilution (10\%), confirming the power of flow cytometry as an accurate means to measure the degree of biofouling.

\subsubsection{Functionalization of pSB-coated beads}

Figure 3.3 shows the excellent antifouling properties of the pSB-coated beads. However, to use such beads for sensing purposes, they need to be combined with specific recognition elements. For this purpose, the terminal bromide that remains at the chain ends after ATRP can be used to incorporate biologically relevant molecules, as was already previously shown on poly(sulfobetaine)-coated silicon nitride surfaces. ${ }^{38}$ To test the ability to modify pSB-coated beads in a similar fashion, several coupling reactions were performed which all aimed for the incorporation of fluorinated compounds - incorporation of fluorine facilitates the monitoring of the surface reactions by XPS, due to the high sensitivity of this technique towards fluorine atoms. Initially, pSB-coated beads were reacted with heptafluorobutylamine (Scheme 3.1, route $\mathrm{c} 1)$. The appearance of a small, but distinct F 1s peak $(686.0 \mathrm{eV})$ (Figure 3.5) and the concomitant disappearance of the Br 3d peak (see Supporting Information, Figure S3.5) in the XPS wide scan of these beads, indicated a successful substitution reaction, which thus confirms the availability of the terminal halide on the coated beads for surface reactions. To investigate the usability of pSB-coated beads for subsequent functionalization by click chemistry, the terminal bromides were first converted into azide moieties using sodium azide (Scheme 3.1, route c2). ${ }^{33}$

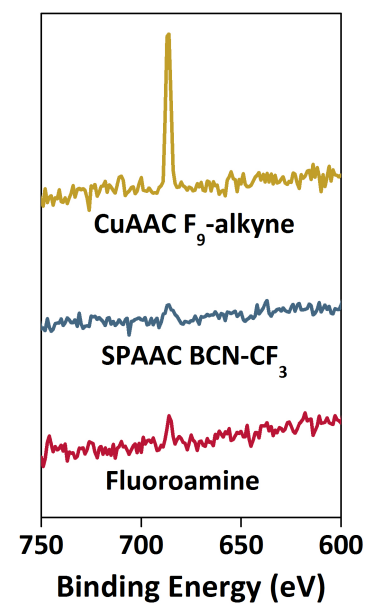

Figure 3.5 F 1s peaks from XPS wide scans obtained from pSB beads substituted with heptafluorobutylaimine, pSB-azide beads functionalized with $\mathrm{BCN}_{-} \mathrm{CF}_{3}$ via SPAAC, and pSB-azide beads functionalized with $\mathrm{F}_{9}$-alkyne via CuAAC. The appearance of $\mathrm{F}$ 1s peaks in these XPS spectra indicates the successful functionalization of the polymer-coated beads with fluorinated model compounds. 
The resulting pSB-azide beads were analyzed by FT-IR (see Supporting Information, Figure S3.9), showing a clear azide signal at $2110 \mathrm{~cm}^{-1}$. The incorporation of azides could be further confirmed by the additional peaks (apart from the amide and ammonium peak) in the XPS N 1s narrow scan (see Supporting Information, Figure S3.4) that could be assigned to the azide moiety, similar to what was previously observed for azides within sulfobetaine brushes (chapter 2).$^{29}$ The pSB-azide beads were then functionalized via the widely-used Copper-Catalyzed AzideAlkyne Cycloaddition (CuAAC) ${ }^{34}$ or Strain-Promoted Alkyne Azide Cycloaddition (SPAAC) ${ }^{29}$ click chemistry reactions using a fluorinated alkyne ( $\mathrm{F}_{9}$-alkyne, Scheme 3.1, route $\mathrm{d} 2$ ) or a $\mathrm{CF}_{3}$-labeled bicyclooctyne ( $\mathrm{BCN}-\mathrm{CF}_{3}$ Scheme 3.1, route $\mathrm{d} 2$ ), respectively. The CuAAC reaction showed the most evident F 1s peak (Figure 3.5). After correcting for the fact that the CuAAC reaction was performed with an alkyne containing nine fluorine atoms while with the SPAAC reaction only three fluorine atoms per molecule were incorporated, the CuAAC reaction was found to be more efficient than the SPAAC reaction (see Supporting Information, Figure S3.6 and Figure S3.7 for wide scans with atomic percentages) under the applied conditions.

The above described results demonstrate that the terminal halogen atoms that are present on the polymer chain ends after ATRP can indeed be used for further functionalization of the polymer-coated beads in a versatile manner. Either by directly substituting the halide with a functional amine, or by first installing an azide moiety on the bead to introduce the appropriate (bio)molecule via click chemistry. As a result, a large variety of different (biological) relevant molecules can be incorporated onto the beads (of which two examples will be described below).

\subsubsection{Specific binding on functionalized pSB beads}

To illustrate the ability to specifically bind a protein target onto the modified beads from a mixture of other proteins, pSB-azide beads were functionalized with BCN-biotin in order to bind streptavidin (Strep-PE). The specific and non-specific binding of fluorescently labeled proteins on the thus prepared pSB-biotin beads was first evaluated by confocal microscopy (Figure 3.6). To this end, non-modified beads were incubated with either BSA-AF488 or Strep-PE, and pSB and pSB-biotin beads were incubated with a mixture of BSA-AF488 $(0.5 \mathrm{mg} / \mathrm{mL})$ and Strep-PE $(50 \mu \mathrm{g} / \mathrm{mL})$. The green channel, visualizing BSA-AF488, clearly showed non-specific binding on non-modified beads (Figure 3.6, left column, top panel). In contrast, no (nonspecific) BSA-AF488 binding was observed on pSB and pSB-biotin-coated beads (Figure 3.6, left column, middle and bottom panel). In the red (PE) channel, no bound Strep-PE was visible except on pSB-biotin beads (Figure 3.6, right column, bottom panel), indicating solely specific binding of Strep-PE to the biotin moieties. Strep-PE did not show any non-specific binding to non-modified beads, which is consistent with the limited amount of bound streptavidin as measured by flow cytometry (see Figure 3.4). Interestingly, it was observed that non-specific binding of BSA-AF488 was rather patchy (better visible in Supporting Information, Figure S3.16), while the specific binding of Strep-PE was more evenly distributed. It has been shown before that serum albumin clusters are formed upon adsorption of this protein onto solid surfaces, especially at high protein concentrations that favor protein-protein interactions. ${ }^{39-40}$ 
Whether the homogenous distribution of streptavidin is a result of specific binding rather than non-specific binding remains elusive.
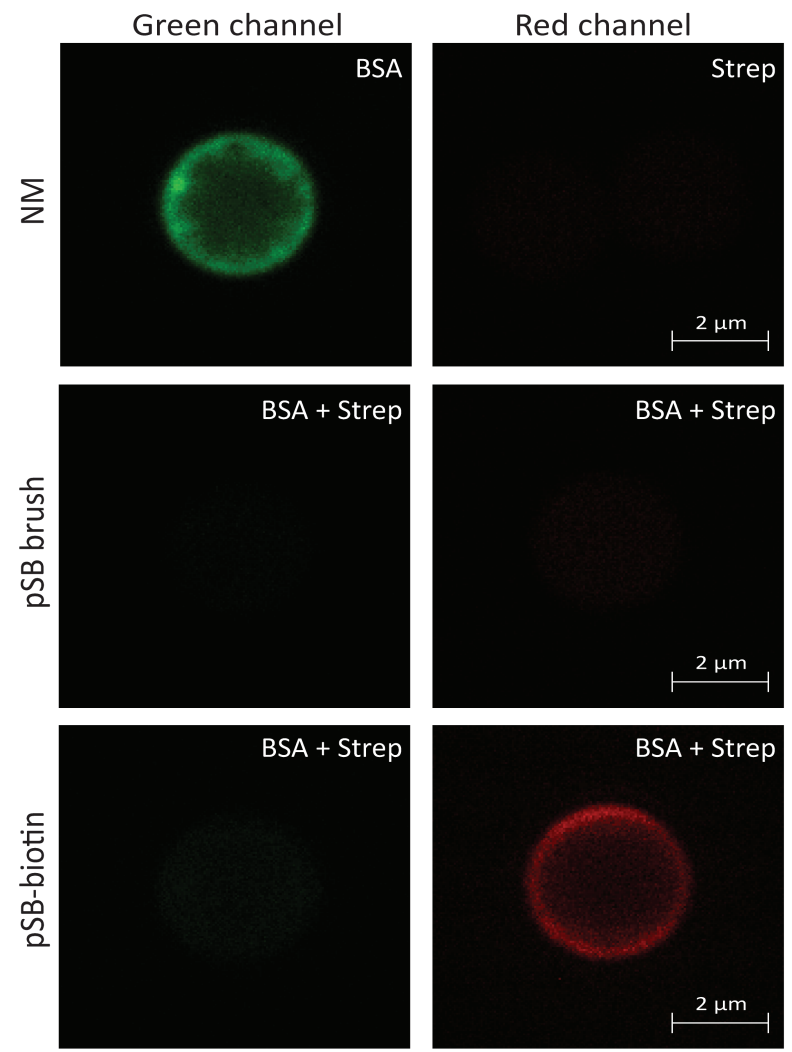

Figure 3.6 Confocal images of non-modified, pSB-coated and pSB-biotin beads incubated with BSAAF488 $(0.5 \mathrm{mg} / \mathrm{mL})$ or Strep-PE $(50 \mu \mathrm{g} / \mathrm{mL})$ or a mixture of the two. The green channel shows the fluorescence as measured with a 488-laser line and 500-531 band-pass filter, the red channel with a 561-laser line with 570-610 band-pass filter.

The pSB-biotin beads were also analyzed by flow cytometry (Figure 3.7), including incubation under the more challenging conditions with Serum-HLF488 solutions. Beads incubated with PBS were again used as a reference. The histogram for PBS (blue), BSA-AF488 (dark green) and Serum-HLF488 (light green) incubated beads are near identical (Figure 3.7A). That is, a shift in fluorescence was practically absent for pSB-biotin beads, irrespective of what protein or protein mixture it was incubated with. This indicates fully retained antifouling properties of the beads after biotin functionalization. On the contrary, a clear shift in fluorescence was observed when the pSB-biotin beads were incubated with the target analyte, Strep-PE (Figure 
3.7B), confirming the results obtained by confocal microscopy. The capacity to specifically bind Strep-PE was not hampered by the presence of a complex mixture of other proteins, as in Figure 3.7B the Strep-PE peak for beads incubated with Serum-HLF488 (magenta) is identical - both in peak width and peak position - to the peak of beads incubated with BSAAF488 (orange). The relative sharpness of the peaks, as compared to beads in PBS, indicates the homogeneous functionalization of the beads with biotin and the subsequent binding of Strep-PE. After incubation at higher streptavidin concentrations, the fluorescence intensity of bound Strep-PE to pSB-biotin beads remained unchanged (see Supporting Information, Figure S3.13), indicating saturation of Strep-PE on those beads. The non-Gaussian distribution of the fluorescence intensity of Strep-PE on pSB-biotin beads might be explained by the tetravalent nature of streptavidin: one streptavidin protein might bind to one or multiple biotin moieties on the bead surface.

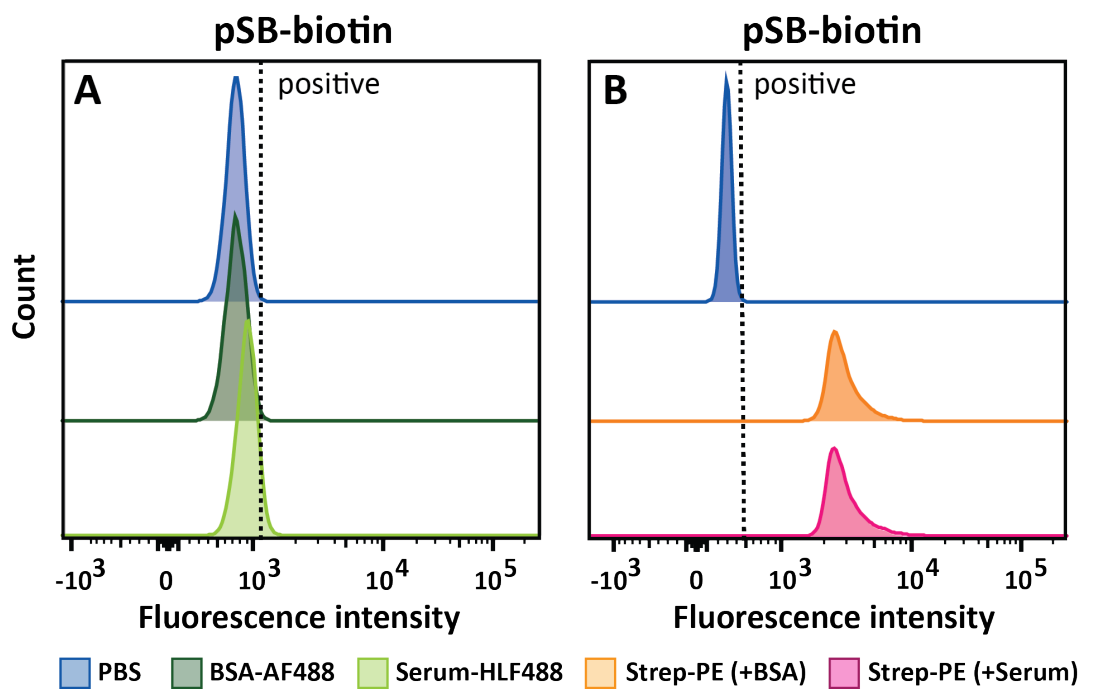

Figure 3.7 Flow cytometry data of pSB-biotin beads incubated with PBS or a mixture of Strep-PE (50 $\mu \mathrm{g} / \mathrm{mL})$ and either BSA-AF4888 $(0.5 \mathrm{mg} / \mathrm{mL})$ or Serum-HLF488 $(10 \%, \sim 6 \mathrm{mg} / \mathrm{mL})$. (A) 488 channel showing BSA-AF488 and Serum-HLF488 binding, PBS (blue) and BSA-AF488 (dark green) overlap, (B) PE channel showing Strep-PE binding, Strep-PE mixed with BSA-AF488 (orange) overlap with Strep-PE mixed with Serum-HLF488 (magenta).

To demonstrate that the use of pSB-coated Dynabeads is not limited to the 'classic', well-known biotin-streptavidin interaction with high affinity, but can also be extended to other more weakly binding interaction partners, we also investigated the ability of pSB beads functionalized with mannose to specifically bind ConA $(50 \mu \mathrm{g} / \mathrm{mL})$ from a mixture with BSA-AF488 or Serum-HL488. To this end, pSB beads were functionalized with propargylamine to first install alkyne moieties on the bead surface (Scheme 3.1, route $\mathrm{c} 3$ ), followed by a CuAAC reaction 
with mannose- $C_{11}$-azide (Scheme 3.1, route d3). Similar to pSB-biotin beads, pSB-mannose beads showed no fouling of BSA-AF488 and only slight protein adsorption when incubated with Serum-HLF488. In contrast, ConA-AF365 was specifically bound by the pSB-mannose beads from both protein mixtures (see Supporting Information, Figure S3.14), although the same level of fluorescence as for Strep-PE on pSB-biotin beads could not be reached. This could be explained by the weaker interaction between ConA and mannose, and/or by a less efficient incorporation of propargylamine or subsequent mannose attachment. Nonetheless, the ability to detect ConA demonstrates the versatility of pSB-coated beads, and illustrates their great potential to be used in sensing applications.

We envision that the here developed bead-based platform may serve multiple purposes as a generic tool to 1) study antifouling materials in a quick and scalable manner (further discussed in chapter 4), 2) investigate the interaction of poorly-understood interaction partners, 3 ) isolate biomolecules (e.g. proteins or cells, further discussed in chapter 5) and 4) serve as a biosensor system that requires stringent antifouling conditions. Billions of antifouling beads are easily prepared at once, which enables not only good statistics (thousands of beads are typically measured per sample versus 1 chip in e.g. SPR analysis, see chapter 7 for a more detailed discussion), but also provides an easy and affordable way of screening reaction conditions (e.g. the effect of certain additives on antifouling behavior). Due to the versatility of the beads with regard to functional group installment, this platform would serve well to investigate yet to be identified or poorly-understood interaction partners. Tailor-made molecules like sugars or peptides attached to the antifouling beads could, in combination with flow cytometry and other techniques, aid to get a better understanding of interacting biomolecules (e.g. which sugar/ glycan is bound by which protein) without background interference. Alternatively, the beads can be used to capture a specific protein (as will be discussed in chapter 5), cell or microbe from a complex pool of other biological entities, to be either used as biosensor platform that requires stringent antifouling conditions or to obtain highly pure biological materials.

\subsection{Conclusion}

We present a novel route towards top-functionalized zwitterionic polymer-coated micronsized beads. These beads show good antifouling properties and are able to specifically bind a target protein, even from a complex mixture of serum proteins. Confocal microscopy and flow cytometry measurements both revealed that non-specific adsorption of BSA and hardly any adsorption of serum proteins occurred on pSB-coated beads, while streptavidin and ConA could be specifically bound to pSB-biotin and pSB-mannose beads, respectively. The ability to discriminate between specific and non-specific binding on many particles simultaneously, together with the high dynamic range of protein detection, makes flow cytometry an excellent technique to study biofouling on micron-sized particles.

The unique combination of excellent antifouling properties and specific binding of target proteins with a routinely used technique as flow cytometry as read-out makes this platform attractive for biosensing applications that require stringent antifouling conditions. Moreover, the 
versatility of the beads with respect to the installment of functional groups via click chemistry (either SPAAC or CuAAC, with azide or alkyne on the bead) or substituting the terminal halide with an amine of choice, also enables these beads to be used for the investigation of a wide range of tailor-made recognition elements.

\subsection{Acknowledgments}

The authors thank Ben Meijer and Jan Willem Borst for their assistance with flow cytometry and confocal microscopy, respectively, and Stefanie C. Lange for stimulating discussions along the process of preparing the antifouling beads. This work was supported by NanoNextNL (program 3E), a micro- and nanotechnology consortium of the government of The Netherlands and 130 partners.

\subsection{References}

1. Reddy, L. H.; Arias, J. L.; Nicolas, J.; Couvreur, P. Magnetic Nanoparticles: Design and Characterization, Toxicity and Biocompatibility, Pharmaceutical and Biomedical Applications. Chem. Rev. 2012, 112, 5818-5878.

2. Haukanes, B. I.; Kvam, C. Application of Magnetic Beads in Bioassays. Biotechnology 1993, 11, 60-63.

3. Morgan, E.;Varro, R.; Sepulveda, H.; Ember, J.A.; Apgar, J.; Wilson, J.; Lowe, L.; Chen, R.; Shivraj, L.; Agadir, A.; Campos, R.; Ernst, D.; Gaur, A. Cytometric Bead Array: A Multiplexed Assay Platform with Applications in Various Areas of Biology. Clin. Immunol. 2004, 110, 252-266.

4. Arruebo, M.; Fernández-Pacheco, R.; Ibarra, M. R.; Santamaría, J. Magnetic Nanoparticles for Drug Delivery. Nano Today 2007, 2, 22-32.

5. Mornet, S.; Vasseur, S.; Grasset, F.; Veverka, P.; Goglio, G.; Demourgues, A.; Portier, J.; Pollert, E.; Duguet, E. Magnetic Nanoparticle Design for Medical Applications. Prog. Solid State Chem. 2006, 34, $237-247$.

6. Jia, G. W.; Cao, Z. Q.; Xue, H.; Xu, Y. S.; Jiang, S.Y. Novel Zwitterionic-Polymer-Coated Silica Nanoparticles. Langmuir 2009, 25, 3196-3199.

7. Yang, W.; Zhang, L.; Wang, S. L.; White, A. D.; Jiang, S. Y. Functionalizable and Ultra Stable Nanoparticles Coated with Zwitterionic Poly(Carboxybetaine) in Undiluted Blood Serum. Biomaterials 2009, 30, 5617-5621.

8. Kim, G.; Yong, Y.; Kang, H. J.; Park, K.; Kim, S. I.; Lee, M.; Huh, N. Zwitterionic Polymer-Coated Immunobeads for BloodBased Cancer Diagnostics. Biomaterials 2014, 35, 294-303.

9. Lin, R.; Li, Y. C.; MacDonald, T.; Wu, H.; Provenzale, J.; Peng, X. G.; Huang, J.; Wang, L. Y.; Wang, A. Y.; Yang, J.Y.; Mao, H. Improving Sensitivity and Specificity of Capturing and Detecting Targeted Cancer Cells with Anti-Biofouling Polymer Coated Magnetic Iron Oxide Nanoparticles. Colloids Surf., B 2017, 150, 261-270.

10. Schlenoff, J. B. Zwitteration: Coating Surfaces with Zwitterionic Functionality to Reduce Nonspecific Adsorption. Langmuir 2014, 30, 9625-9636.

11. Shao, Q.; Jiang, S.Y. Molecular Understanding and Design of Zwitterionic Materials. Adv. Mater. 2015, 27, $15-26$.

12. Prime, K. L.; Whitesides, G. M. Self-Assembled Organic Monolayers - Model Systems for Studying Adsorption of Proteins at Surfaces. Science 1991, 252, 1164-1167.

13. Li, L. Y.; Chen, S. F.; Zheng, J.; Ratner, B. D.; Jiang, S. Y. Protein Adsorption on Oligo(Ethylene Glycol)-Terminated Alkanethiolate Self-Assembled Monolayers: The Molecular Basis for Nonfouling Behavior. J. Phys. Chem. B 2005, 109, $2934-$ 2941. 
14. Ostuni, E.; Chapman, R. G.; Holmlin, R. E.;Takayama, S.; Whitesides, G. M. A Survey of Structure-Property Relationships of Surfaces That Resist the Adsorption of Protein. Langmuir 2001, 17, 5605-5620.

15. Li, L. Y.; Chen, S. F.; Jiang, S.Y. Protein Interactions with Oligo(Ethylene Glycol) (OEG) Self-Assembled Monolayers: Oeg Stability, Surface Packing Density and Protein Adsorption. J. Biomater. Sci., Polym. Ed. 2007, 18, 1415-1427.

16. Ladd, J.; Zhang, Z.; Chen, S.; Hower, J. C.; Jiang, S. Zwitterionic Polymers Exhibiting High Resistance to Nonspecific Protein Adsorption from Human Serum and Plasma. Biomacromolecules 2008, 9, 1357-1361.

17. Vaisocherova, H.; Zhang, Z.; Yang, W.; Cao, Z. Q.; Cheng, G.; Taylor, A. D.; Piliarik, M.; Homola, J.; Jiang, S.Y. Functionalizable Surface Platform with Reduced Nonspecific Protein Adsorption from Full Blood Plasma-Material Selection and Protein Immobilization Optimization. Biosens. Bioelectron. 2009, 24, 1924-1930.

18. Nguyen, A. T.; Baggerman, J.; Paulusse, J. M. J.; van Rijn, C. J. M.; Zuilhof, H. Stable Protein-Repellent Zwitterionic Polymer Brushes Grafted from Silicon Nitride. Langmuir 2011, 27, 2587-2594.

19. Kim, J. C.; Kim, M.; Jung, J.; Kim, H.; Kim, I. J.; Kim, J. R.; Ree, M. Biocompatible Characteristics of Sulfobetaine-Containing Brush Polymers. Macromol. Res. 2012, 20, 746-753.

20. Matyjaszewski, K.; Xia, J. H. Atom Transfer Radical Polymerization. Chem. Rev. 2001, 101, 2921-2990.

21. Jiang, S. Y.; Cao, Z. Q. Ultralow-Fouling, Functionalizable, and Hydrolyzable Zwitterionic Materials and Their Derivatives for Biological Applications. Adv. Mater. 2010, 22, 920-932.

22. Zhang, Z.; Vaisocherova, H.; Cheng, G.; Yang, W.; Xue, H.; Jiang, S.Y. Nonfouling Behavior of Polycarboxybetaine-Grafted Surfaces: Structural and Environmental Effects. Biomacromolecules 2008, 9, 2686-2692.

23. Ning, J. Y.; Li, G.; Haraguchi, K. Synthesis of Highly Stretchable, Mechanically Tough, Zwitterionic Sulfobetaine Nanocomposite Gels with Controlled Thermosensitivities. Macromolecules 2013, 46, 5317-5328.

24. Pochechueva, T.; Chinarev, A.; Bovin, N.; Fedier, A.; Jacob, F.; Heinzelmann-Schwarz, V. PEGylation of Microbead Surfaces Reduces Unspecific Antibody Binding in Glycan-Based Suspension Array. J. Immunol. Methods 2014, 412, 42-52.

25. Kim, G.;Yoo, C. E.; Kim, M.; Kang, H. J.; Park, D.; Lee, M.; Huh, N. Noble Polymeric Surface Conjugated with Zwitterionic Moieties and Antibodies for the Isolation of Exosomes from Human Serum. Bioconjug. Chem. 2012, 23, 2114-2120.

26. Shapiro, H. M. Practical Flow Cytometry 4th ed.; WILEY-LISS: Hoboken, New Jersey, 2003.

27. Jahan-Tigh, R. R.; Ryan, C.; Obermoser, G.; Schwarzenberger, K. Flow Cytometry. J. Invest. Dermatol. 2012, $132,1-6$.

28. Barlogie, B.; Raber, M. N.; Schumann, J.; Johnson, T. S.; Drewinko, B.; Swartzendruber, D. E.; Gohde, W.; Andreeff, M.; Freireich, E. J. Flow-Cytometry in Clinical Cancer-Research. Cancer Res. 1983, 43, 3982-3997.

29. Lange, S. C.; van Andel, E.; Smulders, M. M. J.; Zuilhof, H. Efficient and Tunable Three-Dimensional Functionalization of Fully Zwitterionic Antifouling Surface Coatings. Langmuir 2016, 32, 10199-10205.

30. Debrassi, A.; Ribbera, A.; de Vos, W. M.; Wennekes, T.; Zuilhof, H. Stability of (Bio)Functionalized Porous Aluminum Oxide. Langmuir 2014, 30, 1311-1320.

31. Pujari, S. P.; Spruijt, E.; Stuart, M. A. C.; van Rijn, C. J. M.; Paulusse, J. M. J.; Zuilhof, H. Ultralow Adhesion and Friction of Fluoro-Hydro Alkyne-Derived Self-Assembled Monolayers on H-Terminated Si(111). Langmuir 2012, 28, 17690-17700.

32. Debrassi, A.; Roeven, E.; Thijssen, S.; Scheres, L.; de Vos, W. M.; Wennekes, T.; Zuilhof, H. Versatile (Bio)Functionalization of Bromo-Terminated Phosphonate-Modified Porous Aluminum Oxide. Langmuir 2015, 31, 5633-5644.

33. Li, Y.; Giesbers, M.; Gerth, M.; Zuilhof, H. Generic Top-Functionalization of Patterned Antifouling Zwitterionic Polymers on Indium Tin Oxide. Langmuir 2012, 28, 12509-12517.

34. Hong, V.; Presolski, S. I.; Ma, C.; Finn, M. G. Analysis and Optimization of Copper-Catalyzed Azide-Alkyne Cycloaddition for Bioconjugation. Angew. Chem. Int. Ed. 2009, 48, 9879-9883.

35. Blaszykowski, C.; Sheikh, S.; Thompson, M. Surface Chemistry to Minimize Fouling from Blood-Based Fluids. Chem. Soc. Rev. 2012, 41, 5599-5612.

36. Yang, W.; Chen, S. F.; Cheng, G.; Vaisocherova, H.; Xue, H.; Li, W.; Zhang, J. L.; Jiang, S. Y. Film Thickness Dependence of Protein Adsorption from Blood Serum and Plasma onto Poly(Sulfobetaine)-Grafted Surfaces. Langmuir 2008, 24, 9211 - 
9214.

37. Riedel, T.; Riedelova-Reicheltova, Z.; Majek, P.; Rodriguez-Emmenegger, C.; Houska, M.; Dyr, J. E.; Brynda, E. Complete Identification of Proteins Responsible for Human Blood Plasma Fouling on Poly(Ethylene Glycol)-Based Surfaces. Langmuir 2013, 29, 3388-3397.

38. Nguyen, A. T.; Baggerman, J.; Paulusse, J. M. J.; Zuilhof, H.; van Rijn, C. J. M. Bioconjugation of Protein-Repellent Zwitterionic Polymer Brushes Grafted from Silicon Nitride. Langmuir 2012, 28, 604-610.

39. Cai, Y.; Schwartz, D. K. Influence of Protein Surface Coverage on Anomalously Strong Adsorption Sites. ACS Appl. Mater. Interfaces 2016, 8, 511-520.

40. Langdon, B. B.; Kastantin, M.; Walder, R.; Schwartz, D. K. Interfacial Protein-Protein Associations. Biomacromolecules 2014, 15, 66-74. 


\subsection{Supporting Information}

\subsubsection{Additional XPS spectra}
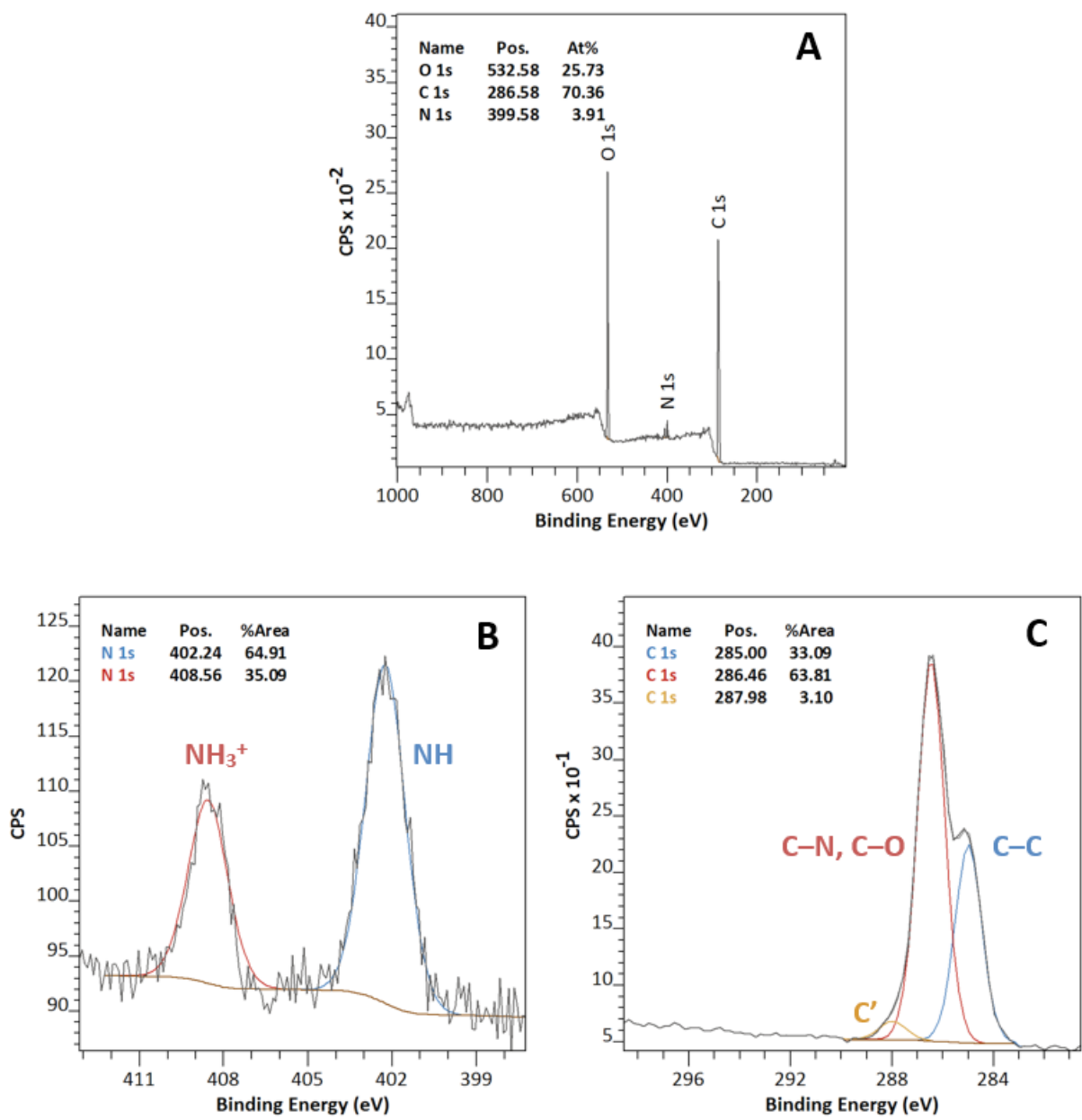

Figure S3.1 XPS spectra of non-modified Dynabeads: (A) wide scan and narrow scans of (B) N 1s and (C) C 1s. The peak labeled as $\mathrm{C}^{\prime}$ is probably from a $\mathrm{C}-\mathrm{O}$ or $\mathrm{C}-\mathrm{N}$ species which originates from the bare Dynabead (as obtained from the supplier) and can also be found in beads modified with an ATRP bromo-initiator (Figure S3.2). 

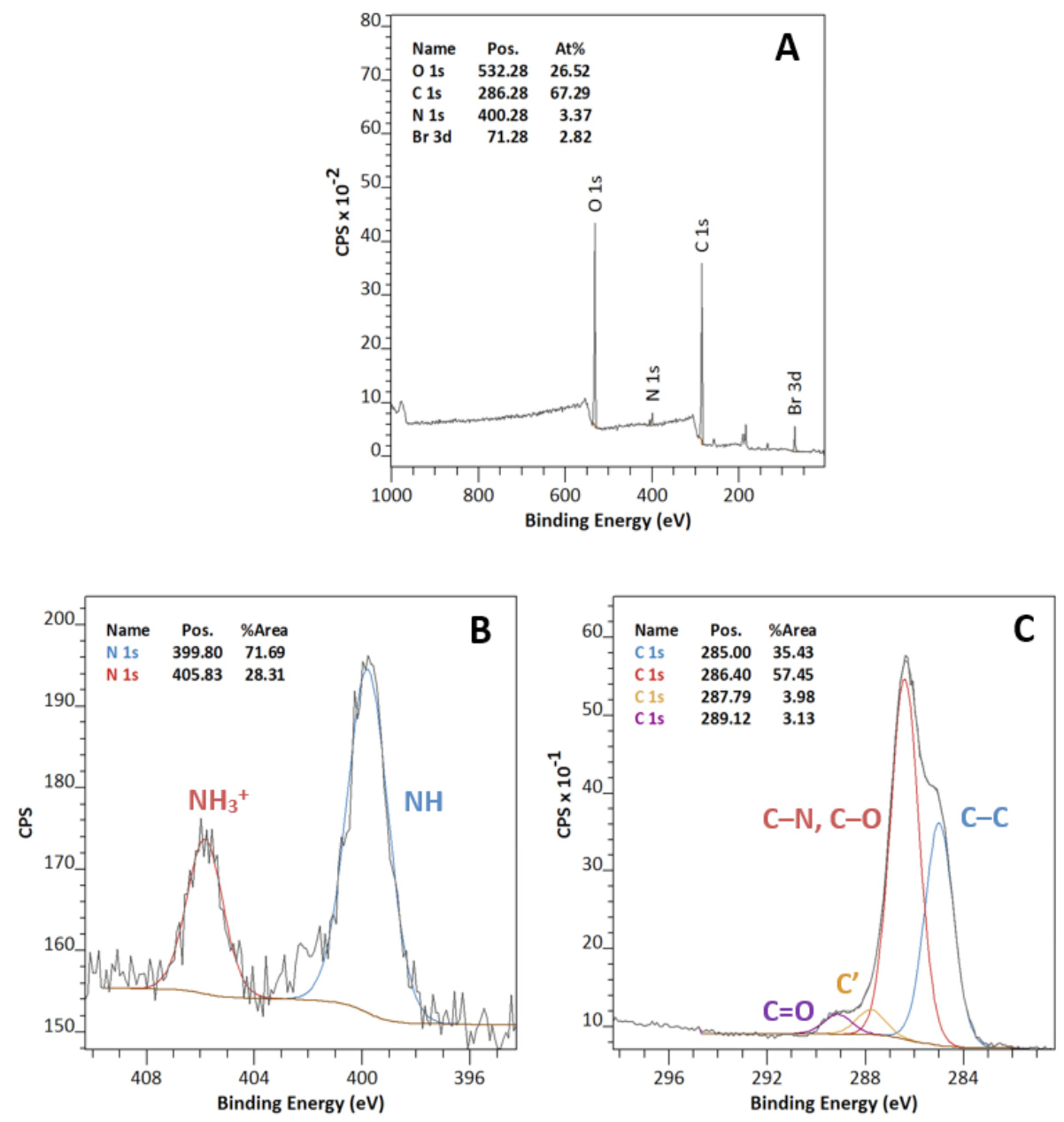

Figure S3.2 XPS spectra of Dynabeads functionalized with $\alpha$-bromoisobutyryl bromide: (A) wide scan and narrow scans of (B) N 1s and (C) C 1s. The peak labeled as C' originates from the bare Dynabead. 


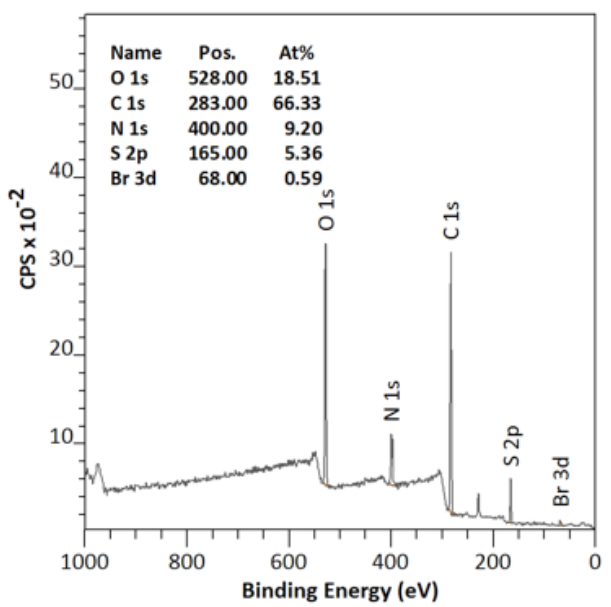

Figure S3.3 XPS wide scan of pSB-coated beads.
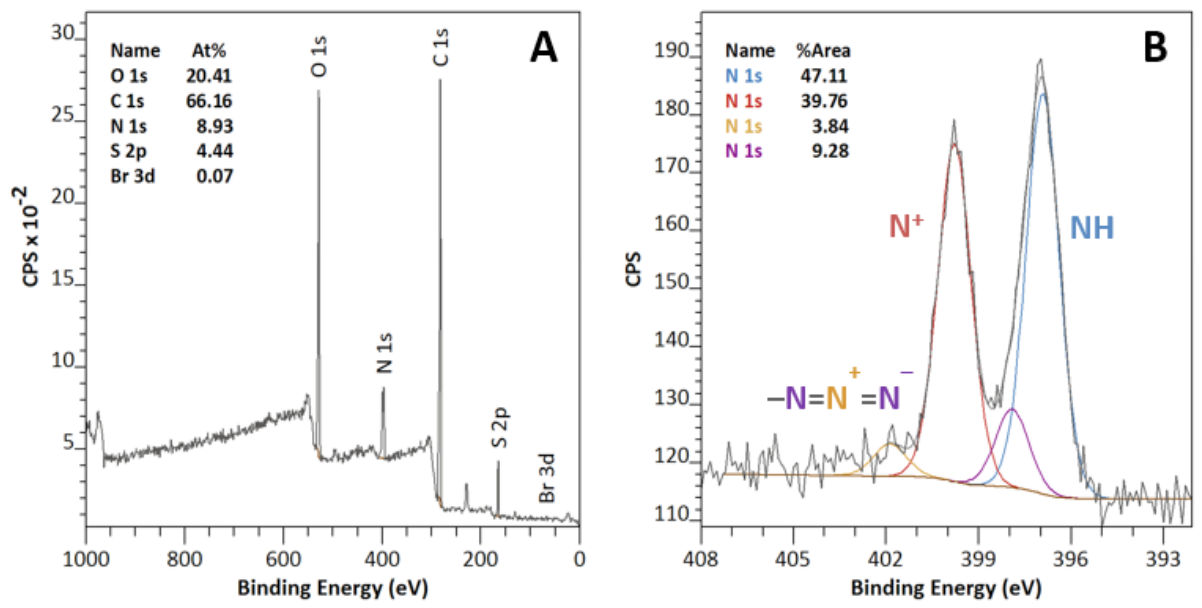

Figure S3.4 XPS spectra of pSB-azide beads: (A) wide scan and (B) N 1s narrow scan. 

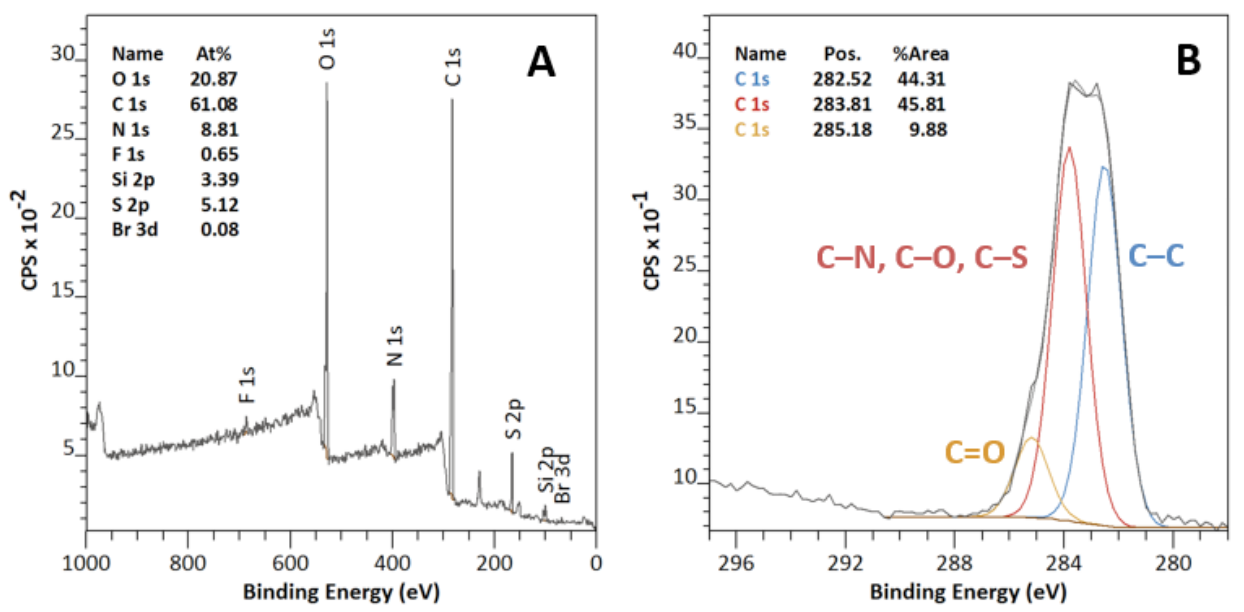

Figure S3.5 XPS spectra of $\mathrm{pSB}^{-\mathrm{F}_{7}}$ beads, as obtained via the substitution reaction between heptafluorobutylamine and the terminal bromide of the pSB-coated beads: (A) wide scan and (B) C 1s narrow scan. 

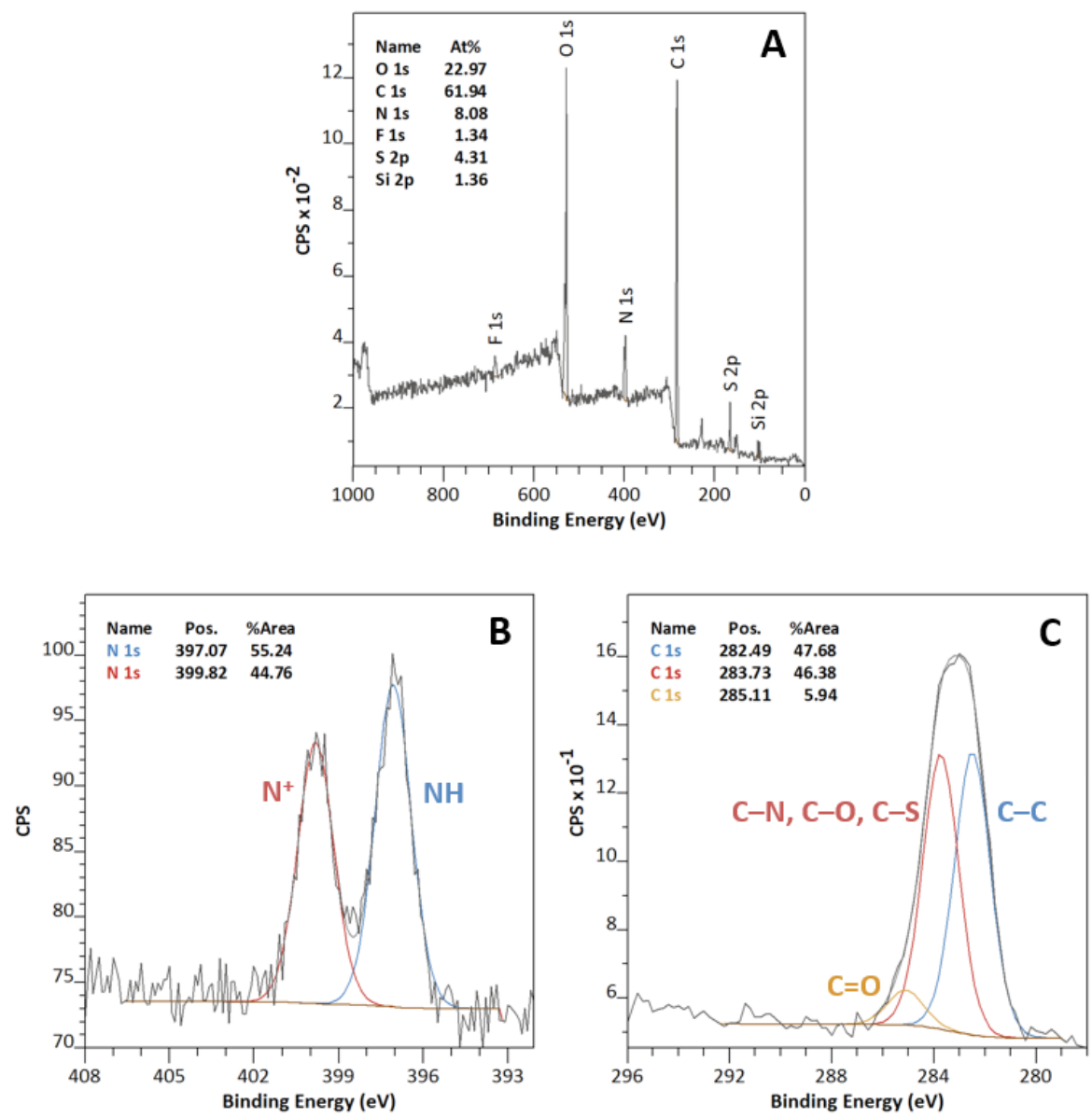

Figure S3.6 XPS spectra of pSB- $\mathrm{CF}_{3}$ beads as obtained via a SPAAC reaction of BCN-CF3 with pSB-azide beads: (A) wide scan and narrow scans of (B) $\mathrm{N} 1 \mathrm{~s}$ and (C) $\mathrm{C} 1 \mathrm{~s}$. 

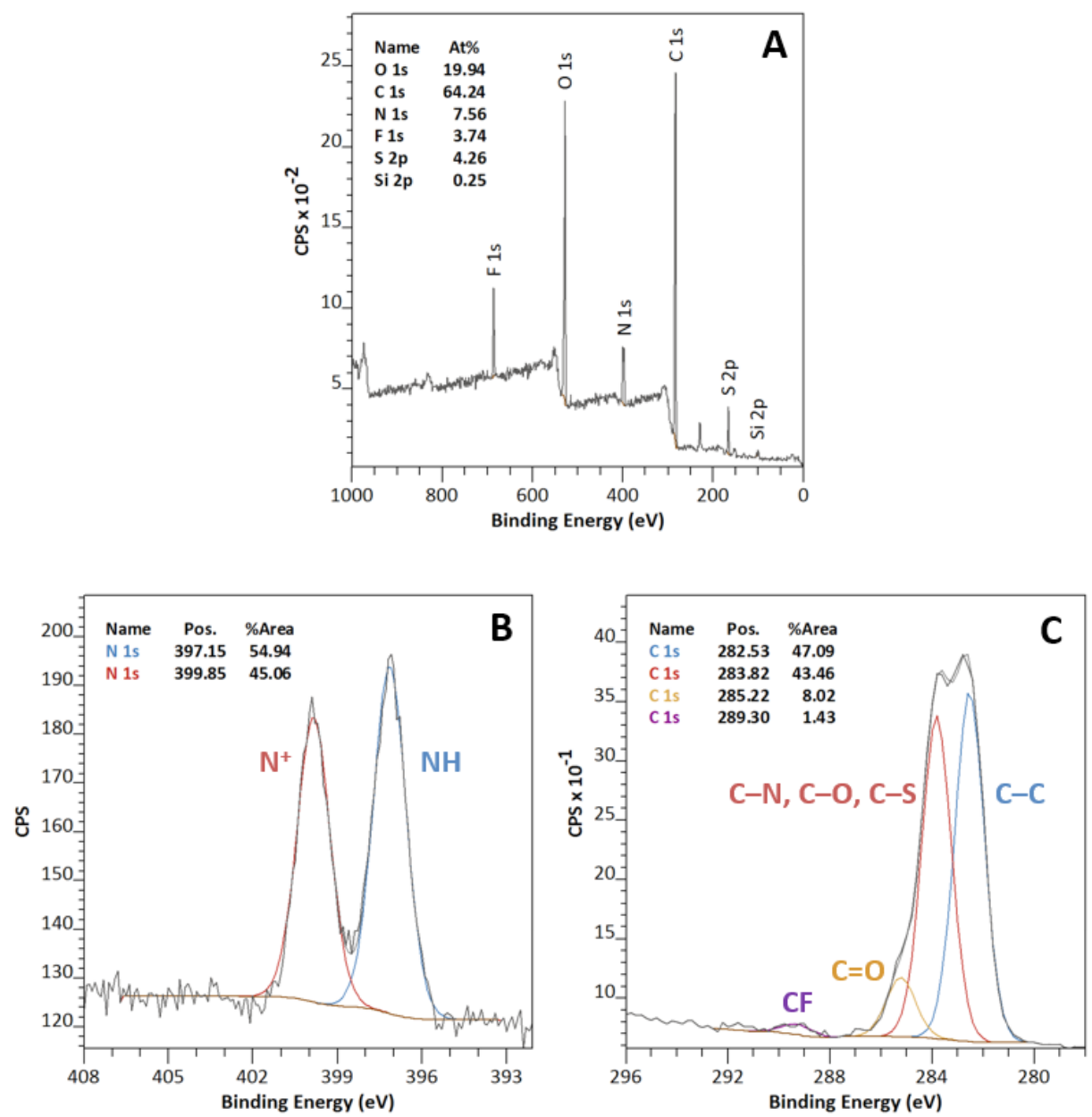

Figure S3.7 XPS spectra of pSB-F, beads as obtained via a CuAAC reaction of the $\mathrm{F}_{9}$-alkyne with pSBazide beads: (A) wide scan and narrow scans of (B) N 1s and (C) C 1s. 

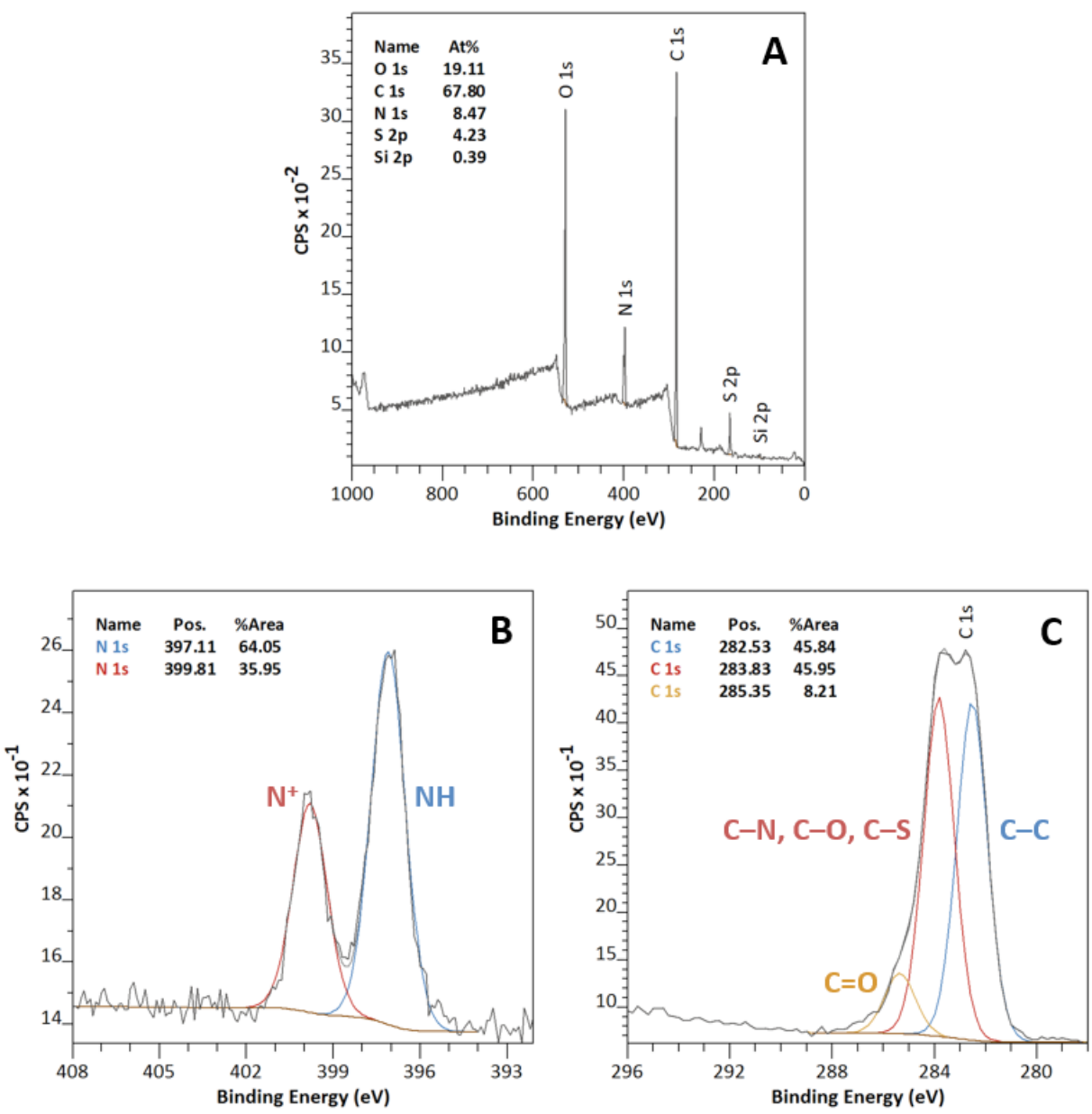

Figure S3.8 XPS spectra of pSB-biotin beads: (A) wide scan and narrow scans of (B) N 1s and (C) C 1s. 


\subsubsection{FT-IR spectroscopy}

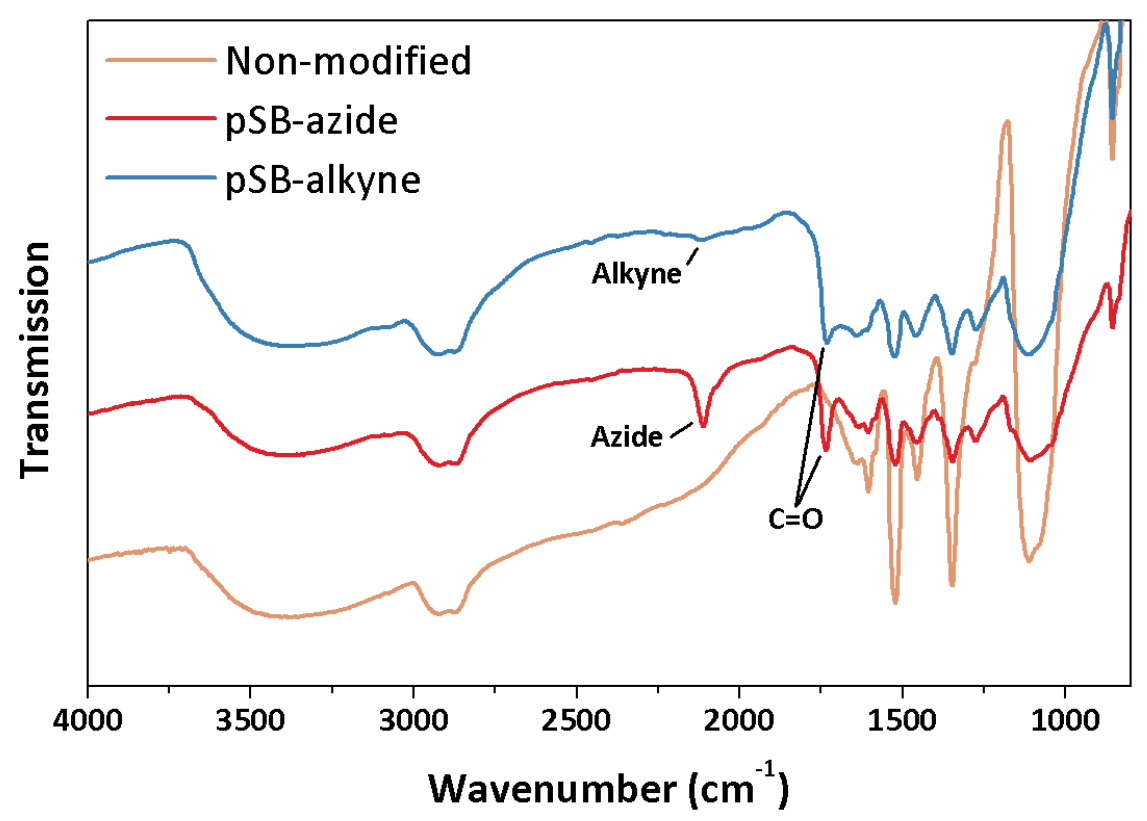

Figure S3.9 FT-IR spectra of non-modified, pSB-azide and pSB-alkyne beads.

Figure S3.9 shows a clear carbonyl peak at $1736 \mathrm{~cm}^{-1}$ for pSB-alkyne and pSB-azide-coated beads that originates from the methacrylamide monomer, while for pSB-azide beads an additional azide peak at $2112 \mathrm{~cm}^{-1}$ is clearly visible. The alkyne absorption band of pSB-alkyne beads could not be convincingly observed (at $2119 \mathrm{~cm}^{-1}$ a very small peak could be seen), most likely due to the intrinsically weak signal of the $\mathrm{C} \equiv \mathrm{C}$ stretch vibration. Moreover, the $\mathrm{C} \equiv \mathrm{C}-\mathrm{H}$ stretch vibration that should appear around $3330-3270 \mathrm{~cm}^{-1}$, overlaps with other signals and could therefore also not be detected. However, subsequent successful CuAAC reactions on the pSBalkyne beads, indicated successful instalment of the alkynes. 


\subsubsection{Additional flow cytometry data}
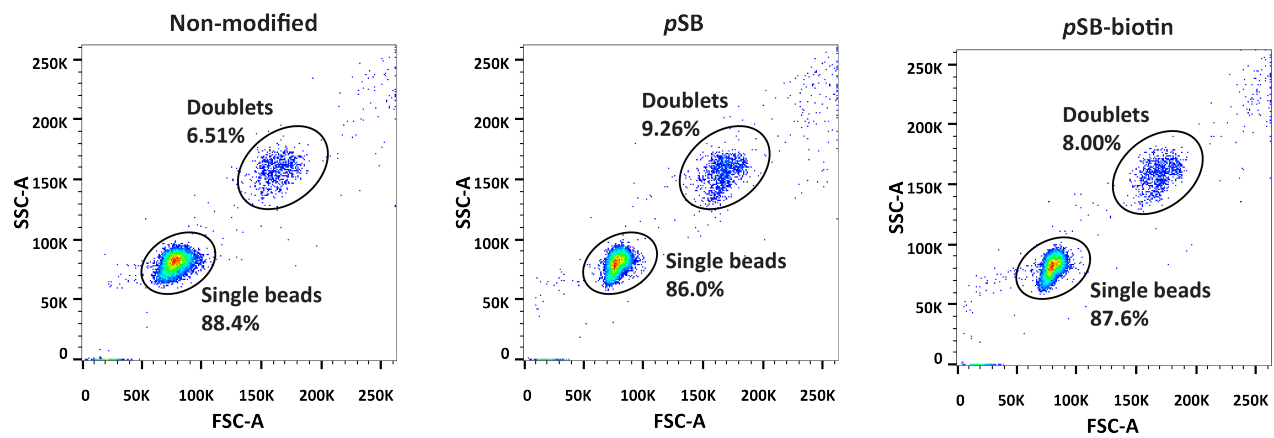

Figure S3.10 Representative gating on non-modified, pSB and pSB-biotin beads after being measured by flow cytometry. 10,000 single beads were measured per sample.

Figure S3.10 shows the dot plots of beads measured by flow cytometry, with on the $x$-axis the forward scatter (FSC) and on the $y$-axis the side scatter (SSC). The forward scatter is a measure for the size of the measured particle, while the side scatter is a measure for the granularity of the particle. Each dot represents one measuring event of a bead or a cluster of beads. A population of beads (beads with similar properties, found roughly within the same area of the dot plot) can be selected by setting the gate (represented here as black circles). For further reading about flow cytometry, the reader is referred to the excellent textbook by Shapiro. ${ }^{\mathrm{S1}}$

Figure S3.10 shows that more than $86 \%$ of the total events are single beads, demonstrating that the measured beads are well dispersed. The percentages of single beads were also very similar for non-modified beads, pSB-coated and pSB-biotin beads. For each measurement 10,000 single beads were measured, and only these single beads were used for further analysis, i.e. each histogram as shown below and in the main text represent single beads only. 

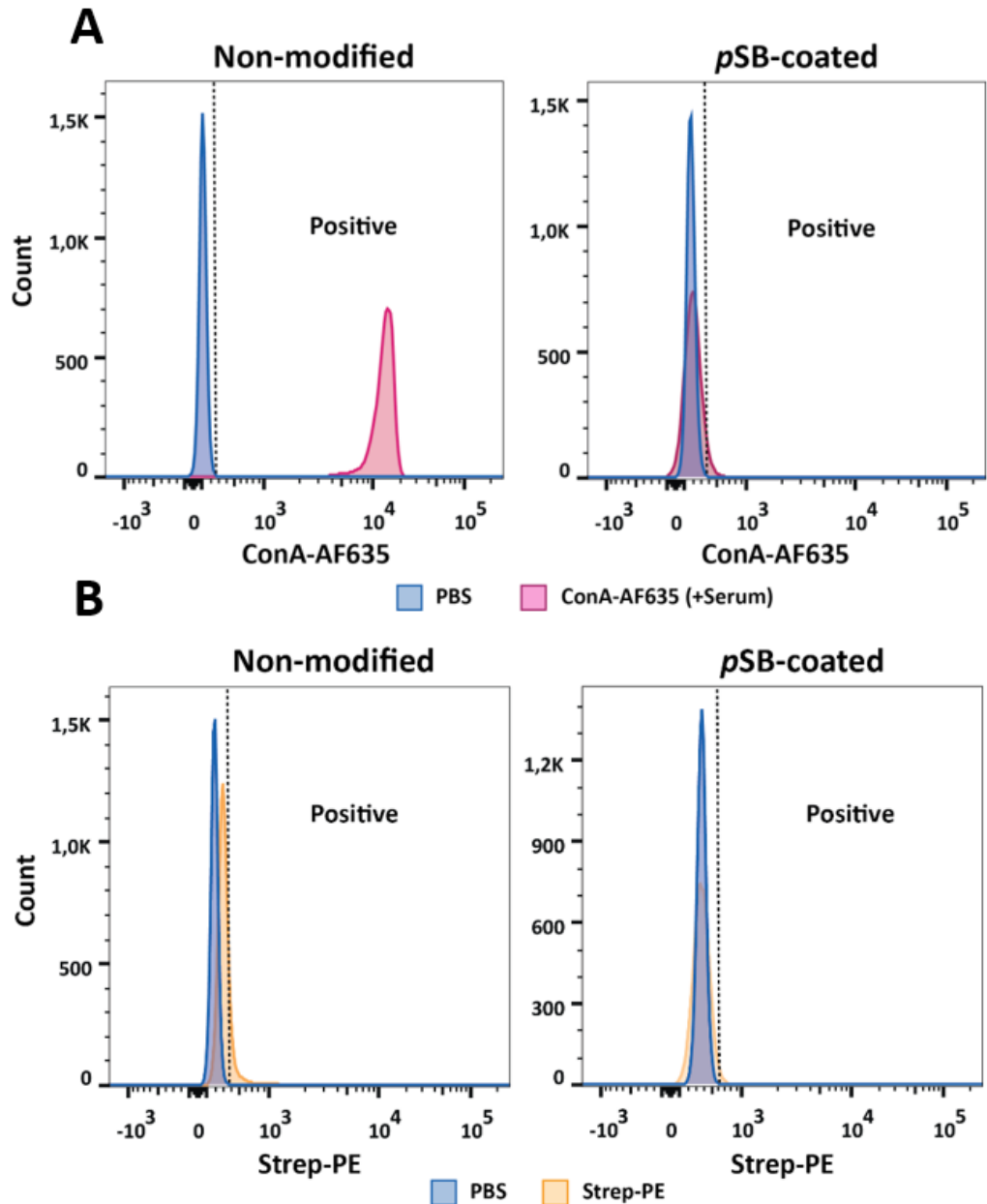

Figure S3.11 Representative flow cytometry data of non-modified and pSB-coated beads incubated with (A) PBS or ConA-AF635 and (B) PBS or Strep-PE. 


\begin{tabular}{|c|c|c|c|}
\hline & solutions & $\begin{array}{r}\text { MFI } \\
\text { BSA-AF488 } \\
\text { Serum-HLF488 }\end{array}$ & $\begin{array}{r}\text { MFI } \\
\text { Strep-PE } \\
\text { ConA-AF635 } \\
\end{array}$ \\
\hline \multirow[t]{7}{*}{ Non-modified } & PBS & 455 & \\
\hline & BSA-AF488 & 34038 & \\
\hline & Serum-HLF488 & 2285 & \\
\hline & PBS & & 343 \\
\hline & Strep-PE & & 454 \\
\hline & PBS & & 258 \\
\hline & ConA-AF635 & & 14053 \\
\hline \multirow[t]{7}{*}{ pSB } & PBS & 791 & \\
\hline & BSA-AF488 & 796 & \\
\hline & Serum-HLF488 & 1063 & \\
\hline & PBS & & 409 \\
\hline & Strep-PE & & 413 \\
\hline & PBS & & 389 \\
\hline & ConA-AF635 & & 450 \\
\hline
\end{tabular}

Table S3.1 Representative flow cytometry data summarizing MFI values of non-modified and pSBcoated beads incubated with PBS, BSA-AF488 (0.5 mg/mL), Serum-HLF488 (10\%, 6 mg/mL), StrepPE $(50 \mu \mathrm{g} / \mathrm{mL})$ or ConA-AF635 $(50 \mu \mathrm{g} / \mathrm{mL})$. Samples using the same gate are clustered.

Inherent to the used method, the MFI of different fluorescently labeled protein solutions cannot be directly compared with respect to the amount of bound protein. For example, there is a significant difference in MFI observed between BSA-AF488 (MFI = 34038) and Serum-HLF488 proteins $($ MFI $=2285)$ and this can most likely be attributed to the different fluorescent properties of the used fluorophores (Alexa Fluor 488 is known to be a highly fluorescent dye) and/or by the degree of fluorophores per protein. 

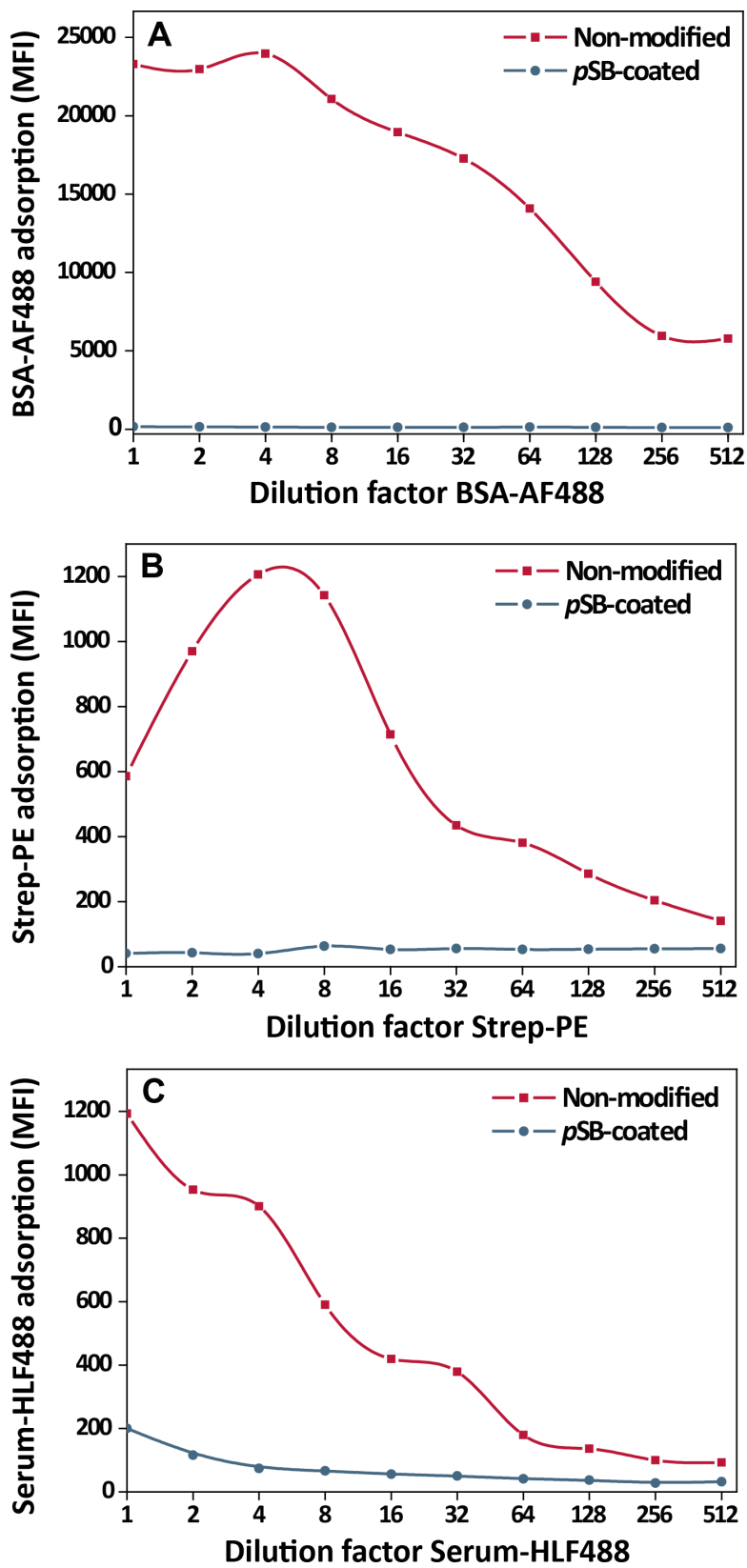

Figure S3.12 Flow cytometry data of pSB and non-modified beads, incubated with (A) BSA-AF488 (highest concentration: $1 \mathrm{mg} / \mathrm{mL}$ ), (B) Strep-PE (highest concentration $100 \mu \mathrm{g} / \mathrm{mL}$ ) or (C) SerumHLF488 (highest concentration: $10 \%$ serum, $\sim 6 \mathrm{mg} / \mathrm{mL}$ ). 


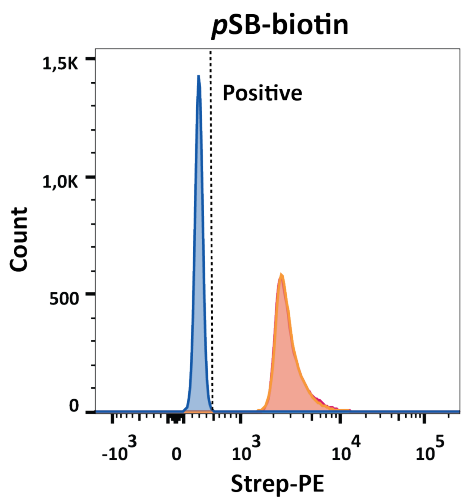

PBS $\square$ Strep-PE $50 \mu \mathrm{g} / \mathrm{mL} \square$ Strep-PE $100 \mu \mathrm{g} / \mathrm{mL}$

Figure S3.13 Flow cytometry data of pSB-biotin beads incubated with either $50 \mu \mathrm{g} / \mathrm{mL}$ or $100 \mu \mathrm{g} / \mathrm{mL}$ Strep-PE. The orange and magenta peaks completely overlap, demonstrating that there is no difference in the amount of Strep-PE binding, despite the difference in concentration.

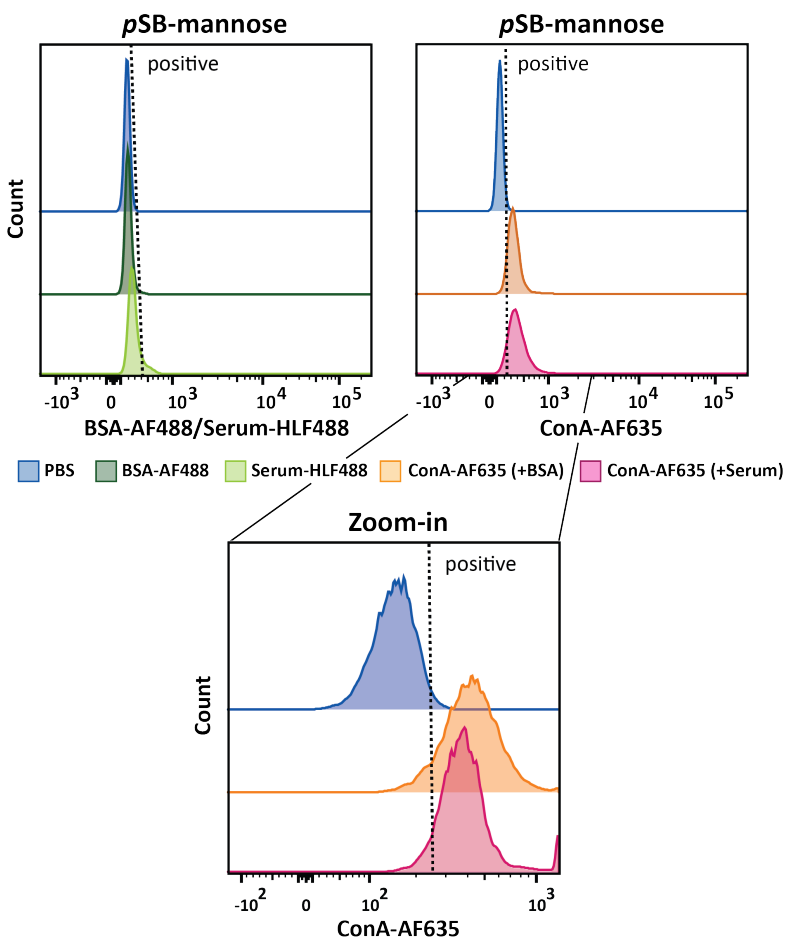

Figure S3.14 Representative flow cytometry data of pSB-mannose beads incubated with PBS, a mixture of ConA-AF635 (50 $\mu \mathrm{g} / \mathrm{mL})$ with either BSA-AF488 (0.5 mg/mL) or Serum-HLF488 (10\%, 6 mg/ $\mathrm{mL})$. 


\subsubsection{Quantification assay}
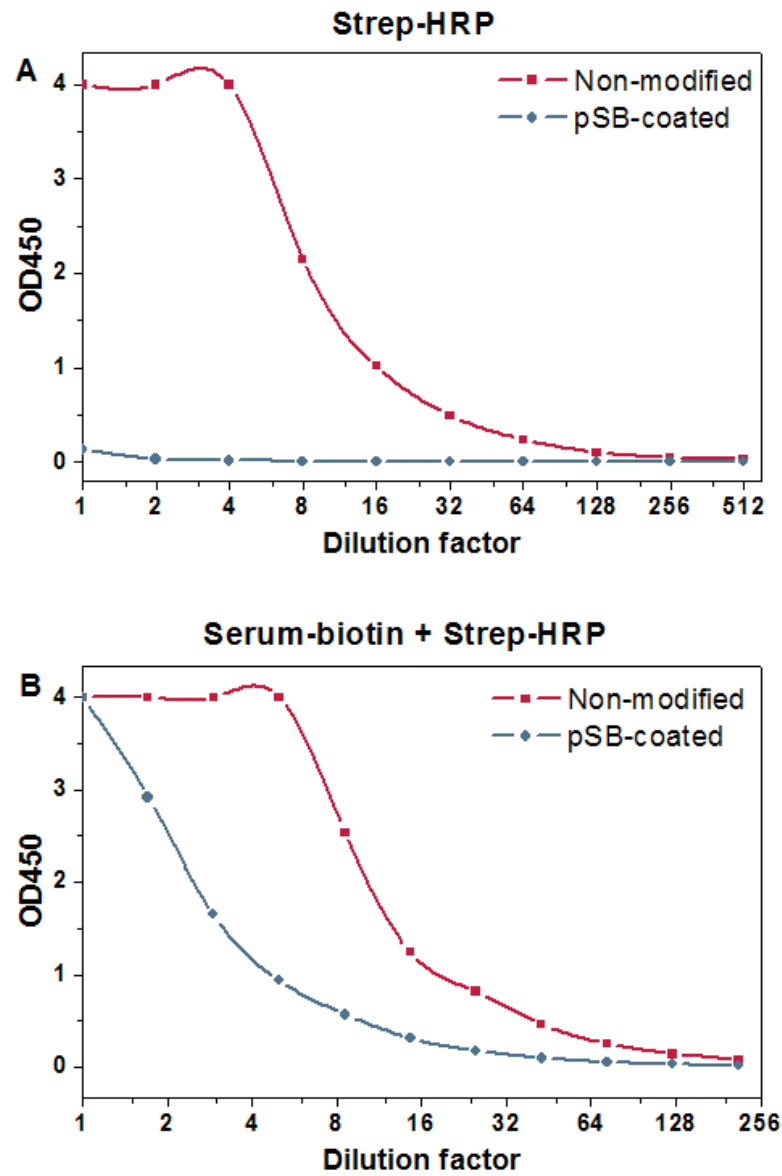

Figure S3.15 HRP quantification assay on non-modified beads and pSB-coated beads, incubated with (A) Strep-HRP and (B) with 10\% biotinylated serum followed by incubation with Strep-HRP. $10 \times 10^{6}$ Beads were incubated with protein solutions, serial dilutions were prepared from these samples before TMB (substrate for HRP) was added and the $\mathrm{OD}_{450}$ was determined. 


\subsubsection{Additional confocal microscopy images}
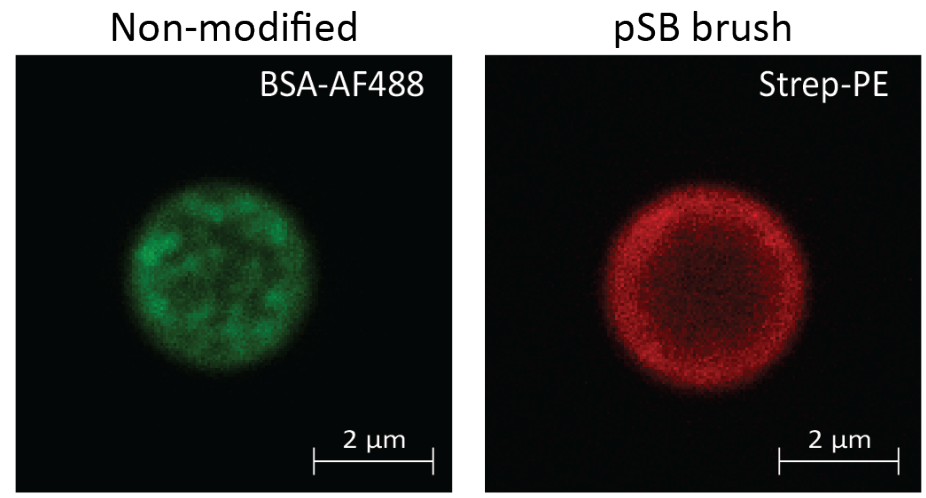

Figure S3.16 Confocal images of non-modified beads incubated with BSA-AF488 and pSB-biotin beads incubated with Strep-PE, both images were taken in such a way that the whole bead is visualized rather than a cross-section of the beads.the amount of Strep-PE binding, despite the difference in concentration.

Figure S3.16 shows the patchy non-specific adsorption of BSA-AF488 proteins on nonmodified beads and the homogeneous binding of Strep-PE to pSB-biotin beads.

\subsection{References}

S1. Shapiro, H. M., Practical Flow Cytometry 4th ed.; WILEY-LISS: Hoboken, New Jersey, 2003. 


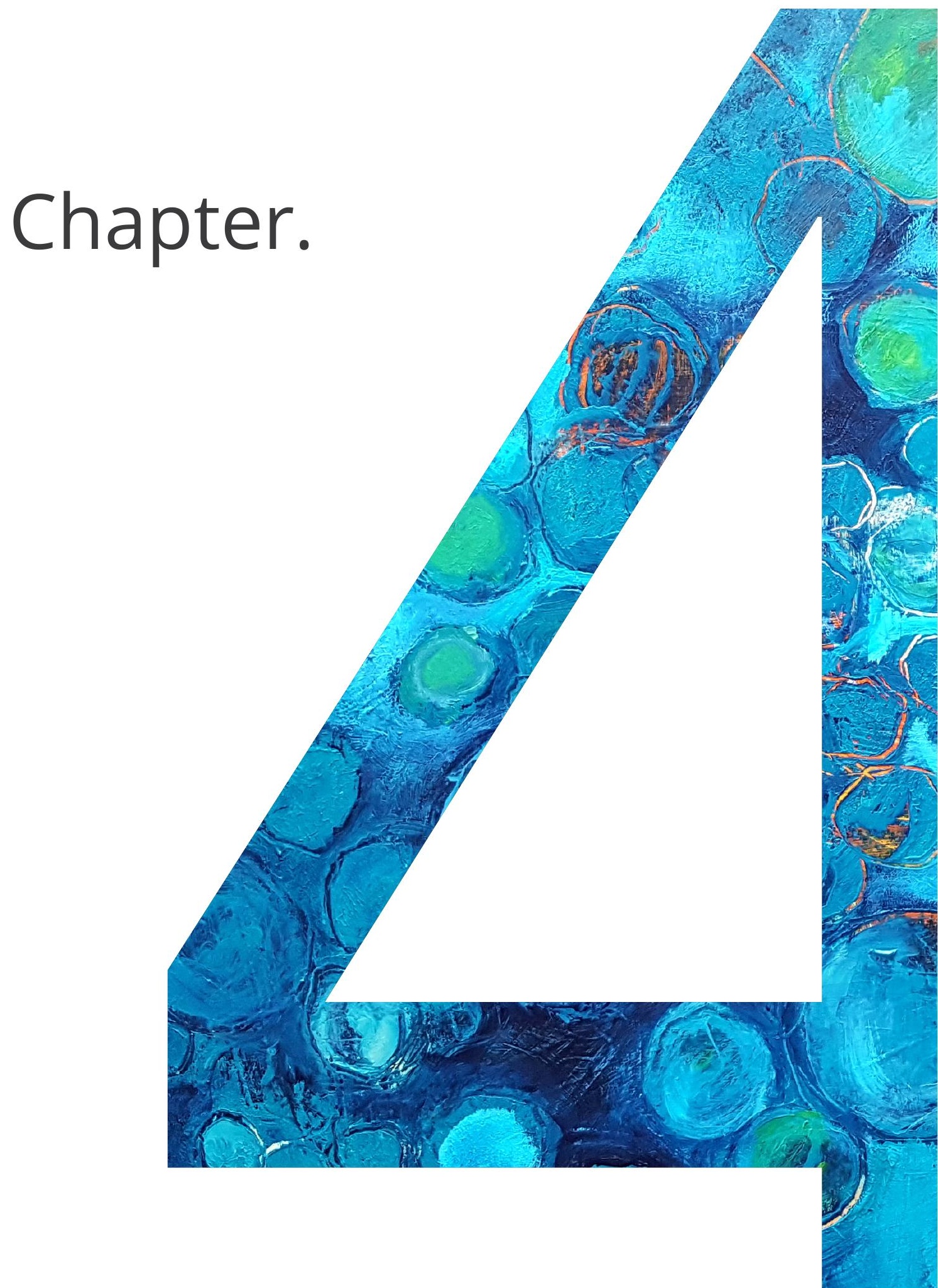




\section{Systematic \\ Comparison of}

\section{Zwitterionic and}

\section{Non-Zwitterionic}

\section{Antifouling Polymer}

Brushes on a Bead-

\section{Based Platform}

van Andel, E.; Lange, S. C.; Pujari, S. P.; Tijhaar, E. J.; Smulders, M. M. J.; Savelkoul, H. F. J.; Zuilhof, H. Systematic Comparison of Zwitterionic and Non-Zwitterionic Antifouling Polymer Brushes on a Bead-Based Platform. Langmuir 2018, DOI: 10.1021/acs.langmuir.8b01832. 


\begin{abstract}
Non-specific adsorption of biomolecules to solid surfaces, a process called biofouling, is a major concern in many biomedical applications. Great effort has been made in the development of antifouling polymer coatings that are capable of repelling the non-specific adsorption of proteins, cells and micro-organisms. In this respect, we herein contribute to the understanding of which polymer brush results in the best antifouling coating. To this end, we compared five different monomers: two sulfobetaines, a carboxybetaine, a phosphocholine and a hydroxyl acrylamide. The antifouling coatings were analyzed using the in chapter 3 described bead-based method with flow cytometry as read-out system. This method allows for the quick and automated analysis of thousands of beads per second, enabling fast analysis and good statistics. We report the first direct comparison made between a sulfobetaine with opposite charges separated by two and three methylene groups and a carboxybetaine bearing two separating methylene groups. It was concluded that both the distance between opposite charges and the nature of the anionic groups have a distinct effect on the antifouling performance. Phosphocholines and simple hydroxyl acrylamides are not often compared with the betaines. However, here we found that they perform equally well or even better, with as overall antifouling ranking: HPMAA $\geq$ PCMA$2 \approx$ CBMAA-2 > SBMAA-2 > SBMAA-3 >> non-modified beads (HPMAA being the best).
\end{abstract}




\subsection{Introduction}

Non-specific adsorption of biomolecules to surfaces is a major concern in many applications, including drug delivery systems, medical implants, and diagnostic devices. ${ }^{1-2}$ Compromised sensitivity of diagnostic tests ${ }^{3}$ and adverse immune responses against drug delivery carriers and indwelling medical devices, ${ }^{4}$ illustrate the great need for effective non-fouling materials.

Two decades ago, Whitesides and co-workers performed a systematic study on the efficiency of different monolayers to suppress protein adsorption, ${ }^{5-6}$ leading to a set of empirical guidelines that is now often referred to as the 'Whitesides rules'. ${ }^{7}$ These guidelines state that good antifouling layers have: 1) polar functional groups, i.e. are hydrophilic, 2) hydrogen bond acceptors, 3) no hydrogen bond donors, and 4) zero net charge. Many different types of antifouling materials have been developed that follow these guidelines of which poly(ethylene glycol) (PEG) based layers are probably the most widely studied and used. ${ }^{8-9}$ Despite their frequent use and ability to prevent protein adsorption from single protein solutions, their antifouling capability is limited when exposed to complex biological media such as blood plasma and blood serum. ${ }^{10}$ As an attractive alternative to PEG, zwitterionic materials have been extensively studied due to their stability in aqueous solutions, ${ }^{11}$ biocompatibility ${ }^{12}$ and excellent antifouling properties, even in complex biological media. ${ }^{10,13}$ Carboxybetaine (CB) and sulfobetaine (SB) monomers are most commonly used to graft zwitterionic brushes from surfaces, because of their commercial availability and straightforward synthesis. ${ }^{14-15}$ Next to that, phosphocholine-based (PC) polymers are the only zwitterionic antifouling materials that are FDA approved and used to enhance the performance of medical devices ${ }^{16}$ and thereby form another important class of antifouling materials. Interestingly, non-zwitterionic materials like simple hydroxyl-containing monomers have also been shown to perform really well, even in complex media, ${ }^{17-19}$ even though these materials do not fully follow the 'Whitesides rules' by being only moderately hydrophilic and containing hydrogen bond donors.

In the last decade, initial systematic studies have attempted to reveal the exact relationship between monomer structure and antifouling performance. ${ }^{10,14,17,20-25}$ These studies showed that even small changes in monomer structure can influence the antifouling performance of the resulting polymer brushes quite significantly. Important factors include, but are not limited to: type of polymerizable group (methacrylate, acrylate, methacrylamide), ${ }^{17}$ nature of the hydrophilic groups (hydroxyl, quaternary ammonium, sulfonate, carboxylate, phosphonate) $)^{10,17,26-27}$ and the carbon spacer length (CSL), ${ }^{14,17,22,24-25}$ which is defined as the number of methylene groups between the cationic and anionic groups (or between hydroxyl and acrylamide). Despite such studies, a general understanding of the ultimate antifouling material has not yet been reached. Most systematic studies either focused on carboxybetaines or sulfobetaines (or a combination thereof) or on hydroxyl-containing polyacrylamides. Direct comparisons of any betaines with the hydroxyl monomers is scarce. In addition, PC-based materials are rarely the topic of systematic studies, probably because of their challenging synthesis. ${ }^{16,28}$ Studies including sulfobetaines typically only consider SB-3 (a sulfobetaine with three methylene groups between opposite charges). Although SB-3 shows good protein 
resistance, it's antifouling efficiency is not as high as observed for CB-2. ${ }^{10}$ This can be explained by their different CSL, and besides that, differences in hydration between the anionic groups in $\mathrm{CB}$ and SB monomers have also been reported. ${ }^{26}$ Two recent studies compared the effect of the CSL in sulfobetaines on their hydration states and fouling behavior for different ionic strength and type of ions. ${ }^{24-25}$ It was concluded that at non-physiological, low ionic strength conditions (i.e. $10^{-3}-10^{-1} \mathrm{M}$, whereas isotonic phosphate-buffered saline (PBS) and blood serum are typically $>0.15 \mathrm{M}) \mathrm{p}(\mathrm{SB}-2)$ surfaces performed less well regarding non-specific protein adsorption than $\mathrm{p}(\mathrm{SB}-3)$. This was attributed to the stronger intra/inter-chain interactions within the $\mathrm{p}(\mathrm{SB}-2)$ brushes, resulting in less hydrated polymer layers. ${ }^{25}$ The fact that $\mathrm{SB}-2$ monomers were, up to our knowledge, never related to SB-3 and CB-2 monomers under the physiological conditions for which zwitterionic materials have been shown to be so useful, prompted us to directly compare polymer brushes derived from the SB-3, SB-2 and CB-2 monomers and thereby deepen our understanding of the structural dependence of monomers on the antifouling performance of resulting polymer brushes. To make a more comprehensive comparison to other main classes of well-performing polymers, polymer brushes derived from a PC and a hydroxyl acrylamide monomer were also included in the study.

Herein, we compare the antifouling performance of polymer brushes derived from two sulfobetaines, SBMAA-3 and SBMAA-2, a carboxybetaine CBMAA-2, a phosphocholine PCMA2, and a hydroxyl acrylamide HPMAA (Scheme 4.1). Polymer brushes were grown and evaluated using our bead-based platform as described in chapter 3. In this method, beads are coated with a polymer brush using standard Surface-Initiated Atom Transfer Radical Polymerization (SI-ATRP) conditions. The adsorption of proteins on the coated beads was evaluated using a purified protein solution (bovine serum albumin (BSA)) and a complex protein solution (bovine serum) and measured using flow cytometry. ${ }^{29}$ The study yielded a clear order of the antifouling capacity of the derived brushes, and detailed insights in the influence of the various factors involved in different media.

\subsection{Materials and methods}

\subsubsection{Materials}

All chemicals and solvents were used without further purification. Acetone (HPLC grade; 99.9\%, BIOSOLVE BV), N,N-dimethylformamide (DMF) (Fisher Scientific), methanol (MeOH) (HPLC grade, Rathburn Chemicals Ltd.), dichloromethane (DCM) (VWR International S.A.S.), isopropanol (HPLC grade; BIOSOLVE), absolute ethanol (EtOH) (Fisher Scientific), dry tetrahydrofuran (Sigma-Aldrich) and deionized water produced with a MilliQ Integral 3 system (Millipore, Molsheim, France) were used as solvents. N-(3-(dimethylamino) propyl)methacrylamide (DMAPMAA) (99\%), sodium 2-bromoethane-1-sulfonate (98\%), $\mathrm{N}, \mathrm{N}$-diisopropylethylamine (DIPEA), copper(I)chloride ( $\geq 99 \%)$, copper(II)chloride $(97 \%)$, copper(I) bromide (99.999 trace metals basis), copper(II) bromide (99.999\% trace metals basis), 2,2'-Bipyridine (99\%), $\alpha$-bromoisobutyryl bromide (98\%), $\beta$-propiolactone, 1,1 -diphenyl- 
2-picryl hydrazyl (DPPH) (97\%), 1,4,8,11-tetramethyl-1,4,8,11-tetraazacyclotetradecane ( $\left.\mathrm{Me}_{4} \mathrm{Cyclam}\right)$ (98\%) and 2-methacryloyloxyethyl phosphocholine (PCMA-2) (97\%) were all purchased from Sigma-Aldrich. 1,3-Propanesultone was ordered from Alfa Aesar (99\%), 11-mercaptoundec-1-yl 2-bromo-2-methylpropionate (MBMP) from Prochimia. Bovine serum albumin-Alexa Fluor 488 conjugate (BSA-AF488) and EZ-Link Sulfo-NHS-LC-Biotin were obtained from Thermo Fisher and streptavidin-phycoerythrin (PE) conjugate from eBioscience. AnaTag HiLyte Fluor 488 micro scale protein labeling kit was obtained from AnaSpec, Inc. PD10 desalting columns were bought from GE Healtcare, flat gold substrates of Au sputtered on glass $(1 \times 1 \mathrm{~cm})$ were purchased from Xantec and magnetic Dynabeads (Dynabeads M-270 amine, $2.8 \mu \mathrm{m}$ in diameter) were purchased from Invitrogen Life Technologies. The $\mathrm{N}$-(2Hydroxypropyl)methacrylamide (HPMAA) monomer was obtained from Polysciences, Inc. Phosphate-buffered saline (PBS) buffer pH 7.4 (5.4 mM Na${ }_{2} \mathrm{HPO}_{4} \cdot 2 \mathrm{H}_{2} \mathrm{O}, 1.3 \mathrm{mM} \mathrm{KH}_{2} \mathrm{PO}_{4}$, $154 \mathrm{mM} \mathrm{NaCl}$ ) was used for all experiments, unless stated otherwise.

\subsubsection{Synthesis of SBMAA-2, SBMAA-3 and CBMAA-2}

\section{SBMAA-3}

3-((3-Methacrylamidopropyl) dimethylammonio)propane-1-sulfonate (SBMAA-3) was synthesized based on previously reported methods (see also chapter 2$).{ }^{30} 12.2 \mathrm{~g}$ (100 mmol) 1,3-Propanesultone was dissolved in $150 \mathrm{~mL}$ acetone and cooled to $0{ }^{\circ} \mathrm{C}$. After adding $10.0 \mathrm{~mL}$ of (55.0 mmol) DMAPMAA, the reaction mixture was allowed to warm up to room temperature and stirred overnight. The resulting precipitate was filtered off and washed rigorously with $500 \mathrm{~mL}$ acetone. After drying under high vacuum, the desired product was obtained as a white powder $(15.1 \mathrm{~g}, 51.7 \mathrm{mmol}, 94 \%)$. The monomer was stored in the fridge at $4{ }^{\circ} \mathrm{C}$. (For detailed characterization, see Supporting Information)

\section{SBMAA-2}

For the synthesis of 2-((3-methacrylamidopropyl)dimethylammonio)ethane-1-sulfonate (SBMAA-2), $5.0 \mathrm{~g}$ DMAPMAA (29 mmol) and $6.2 \mathrm{~g}$ 2-bromoethane sulfonate $(29 \mathrm{mmol}$ ) were added to a $250 \mathrm{~mL}$ round-bottom flask. The reagents were dissolved in $100 \mathrm{~mL}$ DMF under nitrogen atmosphere and vigorous stirring at $60^{\circ} \mathrm{C}$ overnight. Next, $5.1 \mathrm{~mL}$ DIPEA (43 mmol) was added and $2 \mathrm{~h}$ later followed by another equivalent of 2 -bromoethane sulfonate. The reaction mixture was stirred for an additional three days at $70{ }^{\circ} \mathrm{C}$. DMF was removed under reduced pressure and the resulting mixture filtered over a plug of silica using EtOH:DCM $(1: 1)$, followed by flushing with $\mathrm{MeOH}$ to elute the product. A second column using $100 \% \mathrm{MeOH}$ yielded the product as a white solid ( $4.5 \mathrm{~g}, 16 \mathrm{mmol}, 56 \%$ isolated yield). The product was stored in the freezer at $-20{ }^{\circ} \mathrm{C}$. (For detailed characterization, see Supporting Information) 


\section{CBMAA-2}

The synthesis of 3-((3-methacrylamidopropyl) dimethylammonio)propanoate (CBMAA-2) was based on the procedure of Rodriguez-Emmenegger et al. ${ }^{31} 47.2 \mathrm{~g}$ (277 mmol) of DMAPMAA and a small amount diphenylpicryl hydrazyl (DPPH) as inhibitor were dissolved in $250 \mathrm{~mL}$ dry THF and cooled to $0{ }^{\circ} \mathrm{C} .25 .0 \mathrm{~g}$ (347 mmol) of $\beta$-propiolactone was dissolved in $50 \mathrm{~mL}$ of dry THF and dropwise added to the DMAPMAA solution. After $3 \mathrm{~h}$ at $0{ }^{\circ} \mathrm{C}$, stirring was continued for $24 \mathrm{~h}$ at $4{ }^{\circ} \mathrm{C}$. The formed precipitate was filtered off over a glass filter and the filtrate was washed with THF and ether. The product was dissolved in $\mathrm{MeOH}$ and subsequently precipitated in THF to yield a white solid (30.0 g, $124 \mathrm{mmol}, 45 \%$ yield). The product was stored in the freezer at $-20{ }^{\circ} \mathrm{C}$. (For detailed characterization, see Supporting Information)

\subsubsection{Initiator attachment}

\section{AU SURFACES}

Prior to use, Au surfaces (Xantec) were rinsed with EtOH, MilliQ water and again EtOH, followed by drying in an argon stream. The Au surfaces were immersed in a $2.5 \mu \mathrm{L} / \mathrm{mL}$ solution of 11-mercaptoundec-1-yl 2-bromo-2-methylpropionate (MBMP) in absolute EtOH. The immersed surfaces were placed on a shaker $(80 \mathrm{rpm})$ at room temperature for $24 \mathrm{~h} .{ }^{32}$ The initiator-modified surfaces were cleaned by rinsing with, and sonicating in, EtOH, followed by rinsing with EtOH, MilliQ water, EtOH and DCM, and dried in a stream of argon.

\section{BEADS.}

Amine-terminated Dynabeads ${ }^{\mathrm{TM}}$ (M-270 Amine) were functionalized with an ATRP-initiator based on a previously described procedure (see also chapter 2). ${ }^{29}$ In short, $600 \mu \mathrm{L}$ of Dynabeads were dried in a vacuum oven at $50{ }^{\circ} \mathrm{C}$ for at least $2 \mathrm{~h}$. The beads were resuspended in $2 \mathrm{~mL}$ of dry DCM and $0.5 \mathrm{~mL}(2.9 \mathrm{mmol})$ of DIPEA before $0.6 \mathrm{~mL}(4.9 \mathrm{mmol})$ of $\alpha$-bromoisobutyryl bromide was added. The reaction flask was wrapped with aluminum foil and placed overnight on an end-over-end shaker at room temperature. The beads were washed with copious amount of DCM, then washed twice with $1 \mathrm{~mL}$ of isopropanol and twice with $1 \mathrm{~mL}$ of MilliQ water. The beads were used the same day for surface-initiated polymerization.

\subsubsection{Surface-initiated polymerization of SBMAA-3, SBMAA-2, CBMAA-2 and PCMA-2}

Surface-initiated Atom Transfer Radical Polymerization (SI-ATRP) was performed according to previously described procedures ${ }^{29,33}$ with slight modifications. All steps were performed under an argon atmosphere in Schlenk flasks, and solutions were transferred using argon-flushed syringes. 


\section{AU SURFACES}

An isopropanol/MilliQ water mixture (20/80) was degassed by 5 min of sonication and 30 min of argon bubbling. $16.1 \mathrm{mg}(0.16 \mathrm{mmol})$ of an $\mathrm{Cu}(\mathrm{I}) \mathrm{Cl} / \mathrm{Cu}(\mathrm{II}) \mathrm{Cl}_{2}(90 / 10)$ mixture and $54.7 \mathrm{mg}(0.35 \mathrm{mmol})$ 2,2'-bipyridine were dissolved in $7.0 \mathrm{~mL}$ of the isopropanol/MilliQ mixture and stirred for $15 \mathrm{~min}$. From the resulting brown solution, $1.1 \mathrm{~mL}$ was transferred per flask containing $1.00 \mathrm{mmol}$ of the appropriate monomer (292 mg SBMAA-3, $278 \mathrm{mg}$ SBMAA-2, 242 mg CBMAA-2 and 295 mg PCMA-2, respectively). After 15 min of stirring, except for PCMA for which sample handling was performed as quick as possible to avoid polymerization in solution, the reaction mixtures were transferred to the initiator-modified gold surfaces. The surfaces were placed separately and diagonally in Schlenk flasks with the modified surfaces facing downwards, enabling stirring underneath the surfaces and avoiding sedimentation of solids on the reactive side of the chip. The polymerization reactions were carried out for $12 \mathrm{~min}$ at room temperature while the flasks were covered with aluminum foil. The reactions were stopped by rinsing with, and sonicating in, $60{ }^{\circ} \mathrm{C}$ MilliQ water for 5 min. The surfaces were rinsed with acetone and dried in a stream of argon. Surfaces were stored in an argon glovebox until further use.

\section{BEADS}

SI-ATRP on beads was performed similarly as on the above described gold surfaces with the following exceptions: $23.0 \mathrm{mg}(0.23 \mathrm{mmol})$ of the $\mathrm{Cu}(\mathrm{I}) \mathrm{Cl} / \mathrm{Cu}(\mathrm{II}) \mathrm{Cl}_{2}(90 / 10)$ mixture and $78.1 \mathrm{mg}(0.5 \mathrm{mmol})$ 2,2'-bipyridine were dissolved in $8.2 \mathrm{~mL}$ of degassed isopropanol/ MilliQ. To each monomer, $900 \mu \mathrm{L}$ of the Cu-Bpy mixture was added, which was subsequently transferred to a flask containing initiator-modified beads (amount of beads comparable to 100 $\mu \mathrm{L}$ non-modified beads) dispersed in $200 \mu \mathrm{L}$ of the isopropanol/MilliQ mixture. The reaction was carried out in an aluminum-covered Schlenk flask which was placed under a $45^{\circ}$ angle on a shaker $(80 \mathrm{rpm})$. The reaction was stopped by collecting the beads with a magnetic stand (Promega) and washing them with copious amounts of MilliQ, twice with phosphate-buffered saline (PBS pH 7.4), and then again twice with MilliQ. The beads were stored in MilliQ in a refrigerator $\left(4^{\circ} \mathrm{C}\right)$ until further use.

\subsubsection{Surface-initiated polymerization of HPMAA}

SI-ATRP using HPMAA was adapted from a protocol developed by Rodriguez-Emmenegger et al. ${ }^{19}$ All steps were performed under an argon atmosphere and using Schlenk flasks. Absolute $\mathrm{EtOH}$ and initiator-functionalized Dynabeads in $0.5 \mathrm{~mL}$ of EtOH were degassed by performing eight freeze-pump-thaw cycles using liquid nitrogen to freeze and $30{ }^{\circ} \mathrm{C}$ water to thaw. To a mixture of $3.1 \mathrm{mg}(14 \mu \mathrm{mol}) \mathrm{CuBr}_{2}, 9.6 \mathrm{mg}(67 \mu \mathrm{mol}) \mathrm{CuBr}$ and $20.5 \mathrm{mg}(80 \mu \mathrm{mol})$ of $\mathrm{Me}_{4}$ Cyclam, $4 \mathrm{~mL}$ of degassed EtOH was added. After rigorous stirring and a short sonication step, the resulting bright blue reaction mixture was transferred to a flask containing $480 \mathrm{mg}$ (3.4 mmol) of HPMAA and this mixture was stirred until HPMAA was fully dissolved. To the beads and to an initiator-modified gold surface in $0.5 \mathrm{~mL}$ of degassed EtOH, $2 \mathrm{~mL}$ of HPMAA 
solution was added to a final volume of $2.5 \mathrm{~mL}$. The flasks were placed on a shaker (at an $45^{\circ}$ angle, shaking at $80 \mathrm{rpm}$ ) covered in aluminum foil and allowed to react for $2.5 \mathrm{~h}$ at $30{ }^{\circ} \mathrm{C}$. The surface and beads were washed with $\mathrm{EtOH}$, the surface also sonicated in $\mathrm{EtOH}$ for 5 min, and rinsed with MilliQ.

\subsubsection{Surface characterization}

\section{STATIC WATER CONTACT ANGLE (SCA)}

The wettability of modified gold surfaces was determined by automated static water contact angle measurements using a Krüss DSA100 goniometer. Droplets of MilliQ water $(3 \mu \mathrm{L})$ were dispensed on the surface and angles were measured with a CCD Camera using a tangential method.

\section{X-RAY PHOTOELECTRON SPECTROSCOPY (XPS)}

Prior to XPS analysis, modified beads (in MilliQ) were dropcasted on a plasma-cleaned piece of $\mathrm{Si}(111)$ and dried in a vacuum oven at $50{ }^{\circ} \mathrm{C}$ for at least $2 \mathrm{~h} .{ }^{29}$ Dropcasted beads and modified gold surfaces were subsequently analyzed using a JPS-9200 photoelectron spectrometer (JEOL, Japan). The spectra were obtained using monochromatic Al K $\alpha$ X-Ray radiation at $12 \mathrm{kV}$ and $20 \mathrm{~mA}$, with an analyzer energy pass of $10 \mathrm{eV}$ for narrow scans. The obtained spectra were processed using CASA XPS peak fit program (version 2.3.16 PR 1.6).

Polymer brush layer thicknesses on gold surfaces were calculated using the following equation: ${ }^{32,}$ ${ }^{34} \mathrm{~d}=\ln \left(\mathrm{I}_{\mathrm{Au}}\right) / \mathrm{I}_{\mathrm{Au}} \cdot \lambda_{\mathrm{Au}} \cdot \cos \theta$, where $\mathrm{d}=$ thickness (in $\mathrm{nm}$ ) of the organic layer, $\mathrm{I}_{\mathrm{Au} 0}=$ intensity of XPS signal of Au $4 \mathrm{f}_{7 / 2}$ at $83.9 \mathrm{eV}$ (relative to $\mathrm{C} 1 \mathrm{~s}$ signal) in unmodified gold, $\mathrm{I}_{\mathrm{Au}}=$ intensity of XPS signal of $\mathrm{Au} 4 \mathrm{f}_{7 / 2}$ (relative to $\mathrm{C} 1 \mathrm{~s}$ signal) in modified gold, $\lambda_{\mathrm{Au}}=$ effective attenuation length of $\mathrm{Au} 4 \mathrm{f}$ electrons in the organic films (using a value of $3.858 \mathrm{~nm}$ as reported by Petrovykh et al. $\left.{ }^{35}\right), \theta=$ the photoelectron emission takeoff angle relative to the surface normal $\left(10^{\circ}\right)$.

\section{ZETA POTENTIAL}

Zeta $(\zeta)$ potential measurements were performed using a Zetasizer Nano-ZS apparatus (Malvern Panalytical), equipped with a He-Ne laser operating at $633 \mathrm{~nm}$ and a dip cell with palladium electrodes (ZEN1002, Malvern Panalytical). Bead suspensions were prepared in PBS pH 7.4 (1 $\mathrm{mL}$ total), vortexed prior to use, and transferred to disposable cuvettes (PS $2.5 \mathrm{~mL}$, CAT NO. 7590, BRAND). Measurements were performed at $25^{\circ} \mathrm{C}$. The Zetasizer MalvernVer. 7.02 software was used to acquire the data. The voltage was manually set to $4 \mathrm{~V}$ and used in combination with monomodal measurement settings (to allow measurements in PBS). Measurements were performed using 2 min equilibration time, 10-100 runs (automatically determined) per measurement, five subsequent measurements per sample and using Smoluchowski's model to determine the zeta potentials. All measurements were carried out in duplicate. 


\subsubsection{Serum labeling}

Bovine serum was obtained as described in chapter $3 .{ }^{29}$ Bovine blood serum samples were obtained from healthy cows via coccygeal vein sampling. Serum was collected using Vacuette Blood Collection tubes ( $4 \mathrm{ml}$ Z Serum Separator Clot Activator $13 \times 75$ gold cap-gold ring, premium) from Greiner Bio-One. Blood sample collection was approved by the Board on Animal Ethics and Experiments from Wageningen University (DEC number: 2014005.b). Serum samples (from three different cows) were combined, and heated for $30 \mathrm{~min}$ at $56{ }^{\circ} \mathrm{C}$ in a water bath to inactivate any immunologically active complement proteins. Serum samples were stored at $-20{ }^{\circ} \mathrm{C}$ and prior to use thawed and centrifuged at $9000 \times \mathrm{g}$ for $2 \mathrm{~min}$.

Serum proteins were biotinylated using an EZ-Link sulfo-NHS-LC-Biotin reagent, using the manufacturer's instructions. Assuming that the average molecular weight of serum proteins is $70 \mathrm{kDa}, 50$ equivalents of sulfo-NHS-biotin to serum proteins were used. The reaction was carried out at room temperature for $60 \mathrm{~min}$. Non-reacted material was removed using a desalting PD-10 column (GE Healthcare), following the manufacturer's gravitation protocol with PBS as eluent. The concentration of the obtained biotinylated serum (Serum-biotin) was adjusted with PBS to $10 \%$ serum solution (i.e. $\nabla 6 \mathrm{mg}$ total protein $/ \mathrm{mL}$ ).

\subsubsection{Antifouling studies}

\section{FLOW CYTOMETRY}

For each type of polymer-coated beads 3 samples were prepared: beads incubated in PBS, beads incubated in a BSA-AF488 $(0.5 \mathrm{mg} / \mathrm{mL}$ in PBS) solution and beads incubated in $10 \%$ biotinylated serum. To a $2 \mathrm{~mL}$ Eppendorf tube, $200 \mu \mathrm{L}$ of the appropriate solution and $2 \mu \mathrm{L}$ of the appropriate beads dispersion were added and mixed. The tubes were covered with aluminum foil and placed on an end-over-end shaker for $30 \mathrm{~min}$ at room temperature. The beads were collected using a magnetic stand and washed three times with PBS. The beads that were incubated with biotinylated serum were subsequently incubated for 30 min with a streptavidin-phycoerythrin conjugate (Strep-PE, 1:50 dilution), followed by washing three times with PBS. After the last washing step, the beads were resuspended in $0.5 \mathrm{~mL}$ PBS and transferred to FACS tubes. The beads were analyzed with a BD FACS Canto A (BD Biosciences) flow cytometer. Per sample, 10,000 single beads were measured. BSA-AF488 was visualized in the FITC-channel and Strep-PE in the PE channel. Data analysis was performed using FlowJo software V10.

\section{SURFACE PLASMON RESONANCE}

$p$ (SBMAA-2) and $p$ (SBMAA-3) functionalized gold surfaces were glued onto sample holders (Xantec) using super glue gel (Bison). Protein adsorption was measured by SPR using a Biacore 3000 (GE Healthcare) at $25^{\circ} \mathrm{C}$ with a constant flow of $2 \mu \mathrm{L} / \mathrm{min}$. Protein adsorption was 
monitored by first wetting the surfaces by flowing running buffer (PBS) for 30 min followed by injection of a BSA-containing solution $(1 \mathrm{mg} / \mathrm{mL})$ or dialyzed bovine blood serum $(10 \%$, $33 \%$ and $100 \%$ in PBS) for 20 min, followed by washing with running buffer. The response obtained after injection, relative to the starting baseline, was taken as a measure for the amount of protein fouling. To correct for baseline drifts, linear background subtraction was applied (Origin version 8.5) to all obtained sensorgrams.

\subsection{Results and Discussion}

\subsubsection{Synthesis of SBMAA-3, SBMAA-2 and CBMAA-2}

While the phosphocholine (PCMA-2) and hydroxyl methacrylate (HPMAA) are commercially available, the sulfobetaines (SBMAA-3 and SBMAA-2) and carboxybetaine (CBMAA-2) monomers had to be synthesized. Zwitterionic betaines are typically prepared via a ring-opening reaction of a sultone or a lactone with an acrylate or acrylamide like $\mathrm{N}$-(3-(dimethylamino) propyl) acrylamide (DMAPMAA) to yield the desired sulfobetaine or carboxybetaine, respectively. ${ }^{36}$ The carbon spacer length between the opposite charges of the resulting betaines can be tuned by changing the size of the ring. Various one-step ring-opening syntheses have been described with high yield and easy purification. ${ }^{30-31,37}$ SBMAA-3 and CBMAA-2 were synthesized in this fashion using DMAPMAA with 1,3-propanesultone ${ }^{30}$ (as described in chapter 2) and 1,3-propiolactone, ${ }^{31}$ respectively. Following this synthetic approach, a sultone with two methylene groups, a $\beta$-sultone, should yield the SBMAA- 2 monomer. Yet, it has been reported that $\beta$-sultones are too instable to be isolated. ${ }^{38}$ Two alternative approaches have been described to obtain a sulfobetaine with a carbon spacer length of two: the Michael addition of ethenesulfinyl chloride with N,N-dimethylaminoethyl methacrylate, ${ }^{36}$ and the nucleophilic substitution reaction of 2-bromoethanesulfonic acid with DMAPMA. ${ }^{25}$ We improved the latter approach with respect to the yield and purity by adding reactant and base in a stepwise manner, and by performing two columns to subsequently isolate and purify the product. The thus acquired SBMAA-2 monomer was obtained in an improved yield of 56\% at a $5 \mathrm{~g}$ scale. See for characterization the Supporting Information.

\subsubsection{Modification of gold surfaces}

To establish the right polymerization conditions to obtain brushes of equal thickness for each of the monomers, polymer brushes were first grown from gold-coated glass chips, after which the reaction conditions were transferred to functionalize microbeads (Scheme 4.1). Polymer brushcoated gold substrates allowed the determination of wetting properties and layer thicknesses of the grafted polymer brushes. For this purpose, the surfaces were functionalized with an ATRP initiator via the self-assembly of 11-mercaptoundec-1-yl 2-bromo-2-methylpropionate (MBMP) on the gold layer. SI-ATRP was subsequently performed to grow polymer brushes from the surface using SBMAA-3, SBMAA-2, CBMAA-2, PCMA-2 or HPMAA as monomer. The 
zwitterionic brushes were grown following our developed procedure as described in chapter $2,{ }^{33}$ while the $p$ (HPMAA) brushes were grown as described by Rodriguez-Emmenegger and CO-workers. ${ }^{19}$ The XPS wide scan spectra (see also Supporting Information Figure S4.1 A, S4.3A, S4.5A, S4.7A for quantitative analysis), water contact angles and polymer thicknesses of the obtained surfaces are shown in Figure 4.1, while the XPS narrow scan spectra are presented in the Supporting Information (Figure S4.1, S4.3, S4.5, S4.7).

The XPS wide scan spectra showed the expected elements for each type of polymer brush. That is, besides oxygen, nitrogen and carbon for the $\mathrm{p}$ (HPMAA) and $\mathrm{p}$ (CBMAA-2) layers, also phosphorus (133 eV for P 2p, $190 \mathrm{eV}$ for P 2s) for the phosphocholine-based brushes and

\section{Gold surface}
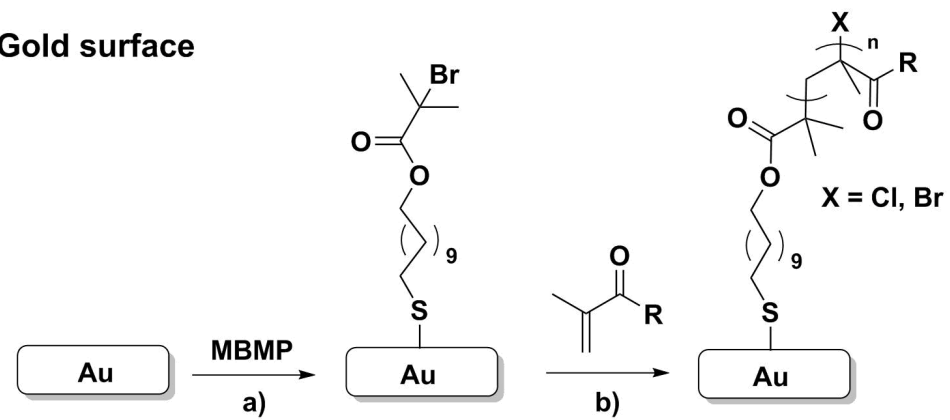

\section{Dynabead}
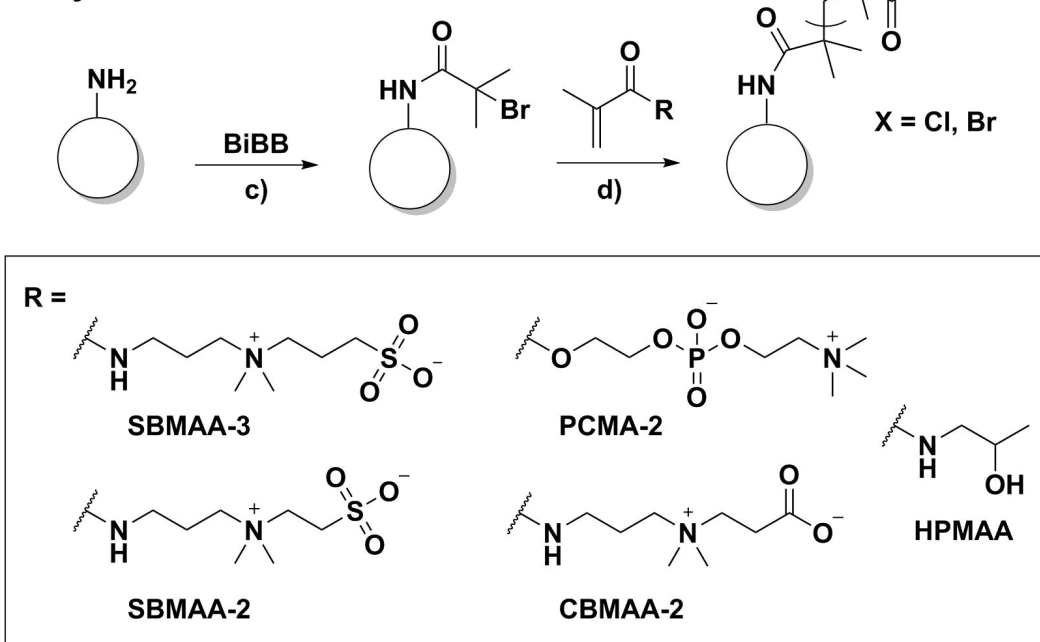

Scheme 4.1 Schematic overview of stepwise polymer brush formation from gold surfaces and dynabeads. (a) MBMP, EtOH, 24 h, RT; (b \& d) for SBMAA-3, SBMAA-2, CBMAA-2, PCMA-2: Cu(I)/ $\mathrm{Cu}$ (II) (90/10), 2,2'-bipyridine, isopropanol/water (20/80), 12 min, RT, for HPMAA: $\mathrm{Cu}$ (I)/Cu(II) (90/10), $\mathrm{Me}_{4}$ Cyclam, EtOH, 2.5 h, $30^{\circ} \mathrm{C}$; (c) $\alpha$-bromoisobutyryl bromide, DIPEA, DCM, overnight, RT. 
sulfur (166 eV for S 2p, $230 \mathrm{eV}$ for S 2s) for the sulfobetaine-based brushes, were detected. Moreover, the carboxy- (Figure S4.5C) and sulfobetaines (Figure S4.1C, S4.3C) showed the characteristic $1: 1$ ratio of the ammonium (401-402 eV) and amide nitrogen (398-399 eV) in the $\mathrm{N}$ 1s narrow scan spectra, confirming the successful growth of intact betaine-based coatings. The $p$ (HPMAA) and p(PCMA-2) brushes showed only one peak in the $\mathrm{N} 1 \mathrm{~s}$ narrow scans (Figure S4.9 and S4.7), which corresponds to either the quaternary ammonium (PCMA2, $401 \mathrm{eV}$ ) or the amide nitrogen (HPMAA, $400 \mathrm{eV}$ ). For all five tested monomers, a distinct carbonyl, $\mathrm{C}-\mathrm{N} / \mathrm{C}-\mathrm{O}$ and $\mathrm{C}-\mathrm{C}$ peak could be observed in the XPS C 1s spectra at 288-289 eV, $286 \mathrm{eV}$ and $285 \mathrm{eV}$, respectively.

In each wide scan spectrum a gold (Au $4 \mathrm{f}$ ) peak was visible at $85 \mathrm{eV}$, allowing us to calculate the thicknesses of the coatings. The zwitterionic polymer brushes were grown under identical conditions and reaction times $(\sim 12 \mathrm{~min})$, which lead in all cases to brush thicknesses between 20-22 nm. Polymer thicknesses of $\sim 20 \mathrm{~nm}$ have been shown to be proficient to obtain excellent antifouling performances. ${ }^{39}$ The polymerization of HPMAA turned out to be non-optimal using our standard polymerization method in water. Therefore a modified procedure using $\mathrm{Me}_{4}$ Cyclam as ligand and EtOH as solvent was used. ${ }^{19}$ Using the $\mathrm{Me}_{4} \mathrm{Cyclam} / \mathrm{EtOH}$ protocol, a layer of $13 \mathrm{~nm}$ was obtained for the p(HPMAA) brushes. It is known that ATRP reactions carried out in less polar solvents $(\mathrm{EtOH})$ are slower than ATRP reactions performed in water, ${ }^{40}$ explaining why after $2.5 \mathrm{~h}$ at $30{ }^{\circ} \mathrm{C}$ thinner brushes were obtained for the HPMAA monomer.

The static water contact angle of the zwitterionic layers were all found to be below $20^{\circ}$, showing their excellent wetting behavior that is often described as key for good antifouling performance. ${ }^{2}$ A water contact angle of $45^{\circ}$ was found for the p(HPMAA) layers, which is in good agreement with previously reported HPMAA-based coatings, ${ }^{19,41}$ suggesting a sufficiently thick brush for good antifouling properties. The contact angle is in line with the hydrophilic nature of the monomer, but, of course, not as low as those obtained for the zwitterionic coatings.

\subsubsection{Modification of microbeads}

Magnetic Dynabeads were coated with p(SBMAA-3), p(SBMAA-2), p(CBMAA-2), p(PCMA-2) or $p$ (HPMAA) brushes via a SI-ATRP procedure that was adapted to beads (as was described in chapter 3), ${ }^{29}$ but similar in terms of concentrations and reaction times compared to the brushes grown on the flat gold surfaces. As the ATRP procedures used on gold surfaces resulted in polymer brushes of similar thicknesses for all monomers, it was assumed that the brushes on the beads will also result in comparable thicknesses for the different monomers. To modify the beads, an ATRP initiator was installed on amine-terminated magnetic Dynabeads via the reaction of the amine groups with $\alpha$-bromoisobutyryl bromide, followed by ATRP for each of the monomers. ${ }^{29}$ XPS wide scan spectra and characteristic narrow scan spectra are shown in Figure 4.1 for each of the polymer-coated beads (for quantitative evaluation of wide scans and additional narrow scan spectra see Supporting Information Figure S4.2, S4.4, S4.6, S4.8, S4.10). 
XPS spectra showed a similar composition for the polymer-coated beads as compared to the coated gold surfaces (with the obvious exception that no Au $4 \mathrm{f}$ peak was seen in the wide scan spectra of the beads). The $p$ (SBMAA-3) and $p($ SBMAA-2)-coated beads displayed two minor
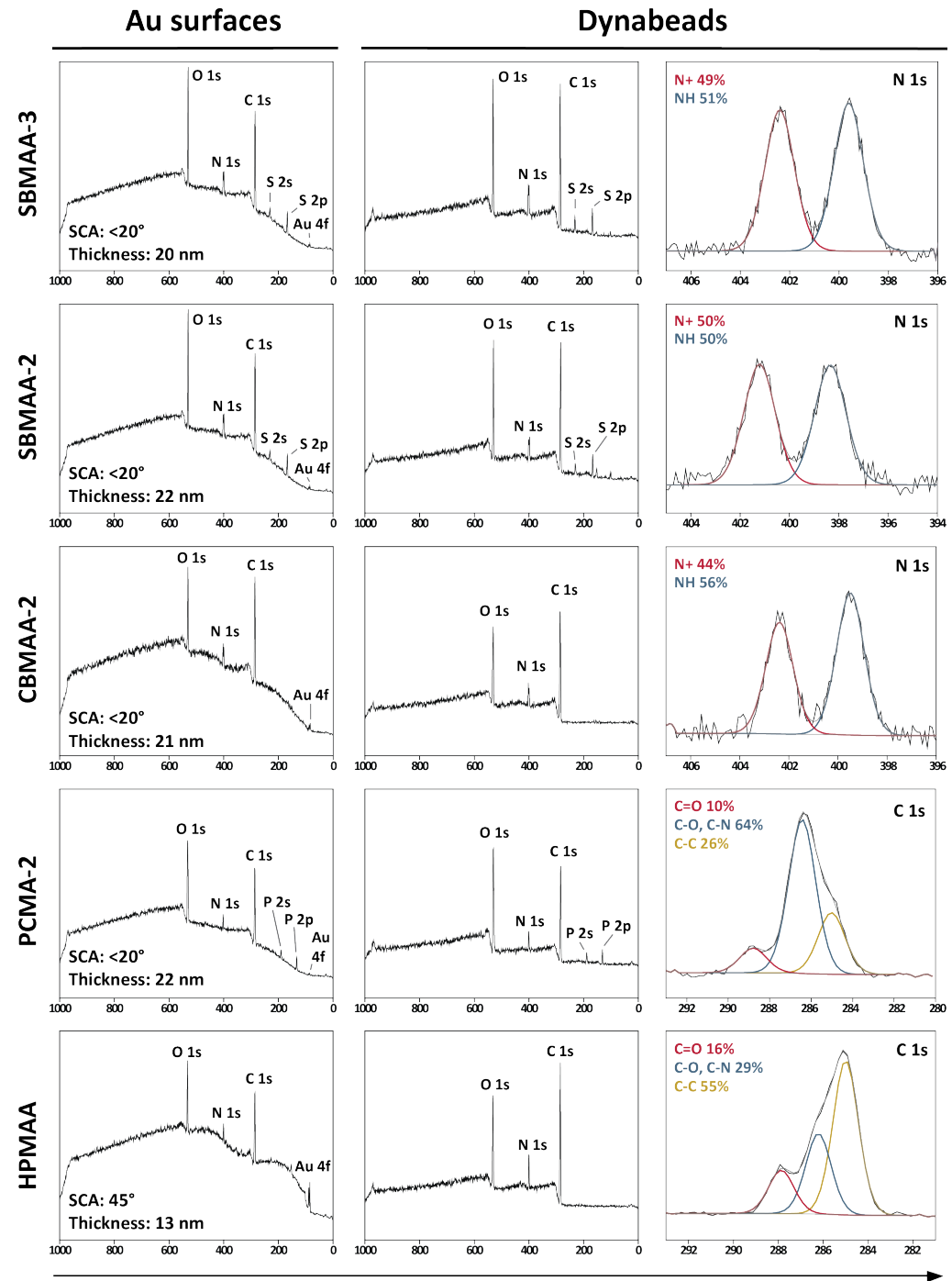

Binding energy (eV)

Figure 4.1 Overview of XPS spectra obtained for gold (Au) surfaces and Dynabeads modified with SBMAA-3, SBMAA-2, CBMAA-2, PCMA-2, or HPMAA polymer brushes, respectively. Reported thicknesses are based on the average of two measurements and based on XPS Au/C ratios. Wide scan spectra and either the $\mathrm{N}$ 1s or $\mathrm{C} 1 \mathrm{~s}$ narrow scan spectrum are depicted for the modified beads. 
silicon peaks at 149 and $100 \mathrm{eV}$, which correspond to the underlying $\operatorname{Si}(111)$ surface on which the beads were deposited for the XPS measurement. The characteristic 1:1 ratio of the nitrogen peaks in the $\mathrm{N}$ 1s spectrum, corresponding to the quaternary ammonium at 401-402 eV and the amide nitrogen at 398-399 eV, were clearly observed for p(SBMAA-3)- and p(SBMAA-2)coated beads.

However, this 1 : 1 ratio was not obtained for $p($ CBMAA-2)-coated beads; at best $44 \%$ ammonium versus $56 \%$ for the amide was found. This is in contrast to the results obtained for the $p$ (CBMAA-2)-coated gold surfaces. It was not caused by an insufficient thickness of the polymer layer, but turned out to be a time-dependent degradation of the p(CBMAA-2) layers within the XPS. This was revealed by XPS analysis of $p($ CBMAA-2) brushes that were grown for $12 \mathrm{~min}$, as in the standard procedure, but in additional experiments also grown for $30 \mathrm{~min}$ and $90 \mathrm{~min}$ on both gold surfaces and beads. However, in none of these the $1: 1$ ammonium to amide peak ratio was observed (see Figure S4.11). Based on the XPS spectra, we speculated that there was an effect of time between sample preparation and XPS analysis on the nitrogen ratio. To evaluate this unexpected observation, the 12 min gold sample was measured twice, without taking the sample out of the ultra-high vacuum (UHV) chamber of the XPS, with $12 \mathrm{~h}$ in between the two measurements. In the second measurement the ammonium peak had become appreciably smaller (see Figure 4.2), indicating that the p(CBMAA-2) coatings degrade over a relatively short period of time within the vacuum of the XPS. This also explains why an intact CBMAA-2 brush could be measured on a gold substrate but not on a bead substrate, as the gold surfaces could be directly measured after preparation while the beads were first dropcasted on a silicon substrate and dried for several hours in a vacuum oven. The disappearance of the ammonium peak was also observed for p(CBMAA-2)-coated gold surfaces upon storage for several days in an argon glovebox (data not shown).

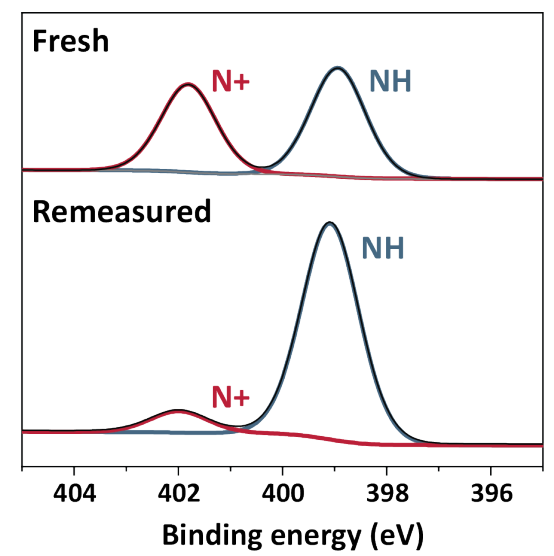

Figure 4.2 XPS N 1s narrow scan of a p(CBMAA-2)-coated gold surface grown via 12 min of SI-ATRP, measured directly after preparation (fresh) and remeasured after $\sim 12 \mathrm{~h}$ (remeasured) within the same XPS measurement (i.e. without taking the sample out of the UHV chamber of the XPS apparatus). 
As the brush thicknesses stayed more or less the same (see Figure S4.12), and the relative nitrogen to carbon-oxygen percentages increased slightly upon storage, we hypothesize that an elimination reaction occurred in which a $\mathrm{C}_{2} \mathrm{H}_{3} \mathrm{COO}^{-}$group is eliminated and a tertiary amine remains on the polymer brush (which thereby loses its quaternary character). It has been reported that CB-2 monomers and resulting polymers are unstable in both acid and base and can undergo a Hofmann elimination. ${ }^{42-44}$ A similar degradation process was not observed for the sulfobetaine or phosphocholine polymer brushes; it is currently not known whether the degradation we observed is specific for the UHV conditions required for the XPS analysis, or whether it also takes place to a significant degree upon prolonged storage in other media. While CB-2-based polymer layers have been established as one of the best antifouling coatings, ${ }^{2}$ this type of degradation has - to the best of our knowledge - never been evaluated in relation to the corresponding antifouling properties. The beads that were used for antifouling studies were kept in an aqueous solution at all steps and did not undergo any drying phase as is required for XPS analysis.

\section{Nonmodified beads}

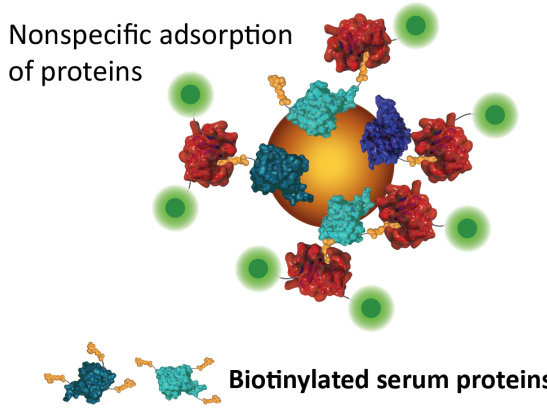

\section{Polymer-coated beads}

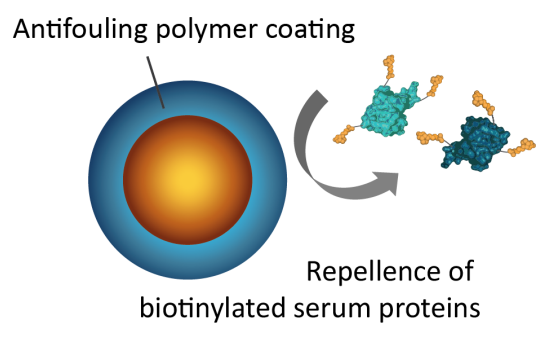

Streptavidin
Fluorescent label

Figure 4.3 Schematic representation of antifouling experiments on non-modified and polymer-coated beads using biotinylated serum proteins. Non-specifically adsorbed serum proteins on the beads are subsequently incubated with fluorescently labeled streptavidin. The fluorescence intensity reflects the amount of adsorped proteins. Polymer-coated beads largely repel the serum proteins and show therefore low fluorescent intensities.

\subsubsection{Antifouling performance}

It was shown in chapter 3 that the antifouling performance and specific binding capabilities of polymer coatings can be reliably evaluated using a bead-based platform with flow cytometry as read-out system. ${ }^{29}$ Flow cytometry allows for the automated measurement of thousands of micron-sized particles per second, enabling quick analysis and good statistics. ${ }^{45}$ Hence, the obtained p(SBMAA-3), p(SBMAA-2), p(CBMAA-2), p(PCMA-2) and p(HPMAA)-coated beads were compared for their antifouling behavior using this platform. To this end, the polymercoated beads were either incubated in PBS, in a solution containing fluorescently labeled 
BSA, or in a biotinylated 10\% serum solution. Beads incubated with biotinylated serum were subsequently stained with fluorescently labeled streptavidin (see Figure 4.3 for a schematic representation). We chose to first biotinylate the serum proteins because a higher fluorescence signal, and therefore sensitivity, could be obtained as compared to the directly fluorescently labeled serum described in chapter $3 .{ }^{29}$ This we rationalize by noting that the serum proteins can be equipped with multiple biotin units, which can all be bound by streptavidin, while each streptavidin can in turn all have multiple fluorescent groups (see Figure 4.3), and by the higher fluorescence of PE compared to HLF-488. The increase in sensitivity enabled us to discriminate a good performing antifouling layer from an excellent one.

For each sample the median fluorescence intensity (MFI) was corrected for the auto-fluorescence of the beads by subtracting the MFI value of a bead sample that was incubated in only PBS (see Table S4.1-Table S4.2 for non-corrected values). The corrected MFI values are depicted in Figure 4.4 Adsorption of more fluorescently labeled proteins onto the beads leads evidently ta a higher fluorescence intensity of those beads. Hence, a higher fluorescence intensity corresponds to a higher degree of fouling. All polymer-coated beads can clearly suppress the non-specific adsorption of proteins as compared to the non-modified (NM) beads (by more than 2 orders of magnitude for BSA and 1 order of magnitude for $10 \%$ serum). In fact, it can be concluded that all polymer-coated beads were able to suppress the adsorption of BSA to levels within the experimental noise. While in itself a useful result, antifouling properties towards single protein solutions do not allow extrapolation towards the antifouling behavior in more complex biological media, like serum solutions. ${ }^{10}$ When the beads were incubated with the $10 \%$ serum solution, differences between the monomers could be observed. These observations lead to the following ranking of antifouling performance based on the amount of adsorbed serum proteins: HPMAA $\geq$ CBMAA-2 $\approx$ PCMA-2 $>$ SBMAA-2 $>$ SBMAA-3 $>>$ non-modified beads.

\subsubsection{Factors determining the antifouling properties of polymer brushes.}

Considering the two sulfobetaine-based coatings, p(SBMAA-2)-coated beads performed better (factor 2.8) in a 10\% serum solution than p(SBMAA-3)-coated beads, which should be attributed to the difference in carbon spacer length between the charges. This is in line with studies that investigated the antifouling behavior of $p$ (carboxybetaines $)^{10,13}$ and $p$ (N-hydroxyl alkyl amide) materials ${ }^{20,46}$ with varying carbon spacer length; reducing the distance between the charges results in improved antifouling properties. Conversely, it was found by Wang et al. ${ }^{25}$ that $p(\mathrm{SB}-3)$, compared to $p(\mathrm{SB}-2)$ and $p(\mathrm{SB}-4)$, is best in preventing non-specific BSA adsorption at non-physiologically low ionic strengths, while at salt concentrations $>0.1 \mathrm{M}$ no differences were seen in BSA adsorption. It has been shown that the behavior of $p(\mathrm{SB})$ materials is highly dependent on the ionic strength as the intra/inter-chain associations between sulfobetaine units can be disrupted by the addition of salt, leading to swelling of the polymer brush. ${ }^{24-26}$ The combination of our data and literature data leads to the following picture: with smaller CSL (less hydrophobic methylene moieties) the interaction between oppositely charged groups is stronger. This charge-charge interaction can be weakened by increasing the salt concentration. 


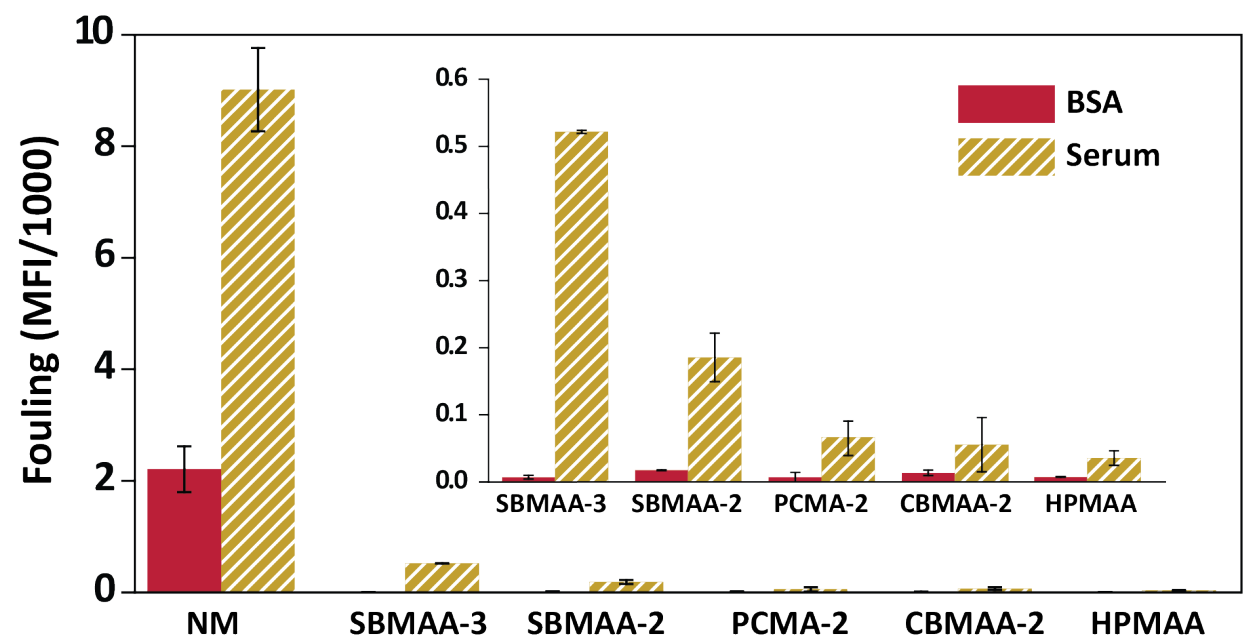

Figure 4.4 Antifouling performance of polymer-coated Dynabeads measured by flow cytometry. Nonmodified beads (NM) and polymer brush-coated beads were either incubated with fluorescently labeled BSA $(0.5 \mathrm{mg} / \mathrm{mL})$ or biotinylated serum $(10 \%$ serum solution, $\sim 6 \mathrm{mg} / \mathrm{mL})$ and fluorescently labeled streptavidin. The median fluorescence intensity (MFI) of the beads was corrected for the MFI values obtained for beads incubated in PBS. Presented data are averages from at least duplicates of independently performed experiments. Standard deviations are presented as error bars. The inset shows a zoom-in of the same data.

For larger CSL, only a small amount of salt is required, while for e.g. SBMAA-2 higher ionic strengths will be required. Once swelling takes places, the hydration of such closely spaced charged (as in p(SB-2) brushes) is also increased, and as a result such brushes are more diffuse and swollen than $\mathrm{p}(\mathrm{SB}-3)$ brushes at $100 \mathrm{mM} \mathrm{NaCl}$ concentration. This suggests that in the low ionic strength regime $p$ (SB-3) brushes are more swollen/better hydrated than $p(\mathrm{SB}-2)$ brushes, which is in line with the finding that their antifouling characteristics under these conditions are better than $p(\mathrm{SB}-2)$ (as indeed found by Wang et al. ${ }^{25}$ ). While relevant for a mechanistic understanding, this regime is of little practical importance, as in most biologically relevant samples the ionic strength is $>0.1 \mathrm{M}$. In contrast, $\mathrm{p}(\mathrm{SB}-2)$ brushes get more swollen than $p(\mathrm{SB}-3)$ brushes in the $>0.1 \mathrm{M}$ salt regime and should therefore perform better at higher salt concentrations (Figure 4.4). To study the differences between $p(\mathrm{SB}-2)$ and $p(\mathrm{SB}-3)$ in more detail, the difference between SBMAA-3 and SBMAA-2 was also evaluated using surface plasmon resonance (SPR), a commonly used method to study antifouling. Figure 4.5 confirms that, when using $3.3 \%, 10 \%$ or $33 \%$ serum solutions, SBMAA-2 consistently outperforms SBMAA-3 in terms of antifouling performance. In addition, the relative difference is bigger for more dilute samples, and like in the bead assay at $10 \%$ serum SBMAA- 2 adsorbs about $3 \times$ less protein than SBMAA-3 (see also Figure 4.4). This also shows the relative quantitative agreement between these techniques. 


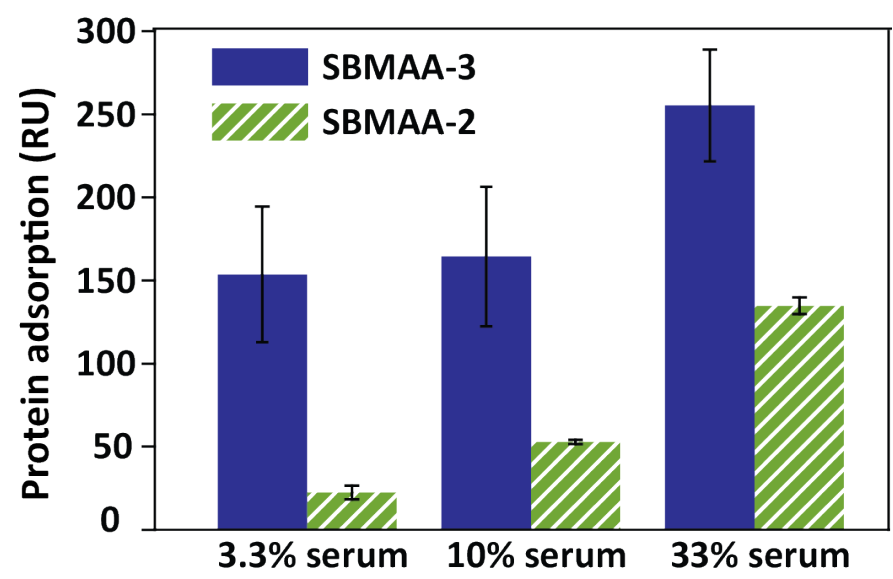

Figure 4.5 Protein adsorption from 3.3\%, 10\% or 33\% cow serum on p(SBMAA-3)- and p(SBMAA2)-coated gold surfaces as measured by SPR. Averages of three independent measurements are shown, error bars represent standard deviations of those measurements. The SPR data were obtained using dialyzed serum solutions as otherwise artefacts were seen in the sensorgrams caused by differences in salt concentration (see Supporting Information for further explanation).

Besides the comparison between SBMAA-2 and SBMAA-3, Figure 4.4 also reveals the differences between SBMAA-2 and CBMAA-2; these data constitute - to the best of our knowledge - the first direct experimental comparison of the antifouling behavior of polymer brushes derived from SB-2 or CB-2 monomers. Figure 4.4 shows that $\mathrm{P}(\mathrm{CBMAA}-2)$ performs better than $p$ (SBMAA-2) in a 10\% serum solution. As the chemical structures of CBMAA-2 and SBMAA-2 are the same except for their anionic group, it can be concluded that as anionic group carboxylates outperform sulfonates. Sulfonate anions are larger than carboxylates, and have their negative charge distributed over more oxygen atoms. As a result, it is expected that more water will surround sulfonates than carboxylates, but that water will be more strongly bound by carboxylates. This qualitative picture has indeed be simulated accurately by Jiang and co-workers. ${ }^{47}$ Together this suggests that a material with few but tightly bound water molecules is more effective in resisting non-specific adsorption of proteins than a material with more water molecules that are loosely bound. To further asses the properties of the polymer brushes, the zeta potential of the polymer-coated beads was also measured, see Figure S4.13. All polymer-coated beads yielded moderately negative zeta potentials, as was previously reported for zwitterionic particles. ${ }^{48-50}$ The zeta potential for p(SBMAA-3), p(SBMAA-2) and $p($ CBMAA-2) are within the same range: $-9.7 \pm 0.7,-8.2 \pm 1.1$, and $-11.1 \pm 0.6$, respectively. Given the similarity in their zeta potential, the observed difference in antifouling capacity of the beads cannot be accounted for by differences in the surface charge of the sulfobetaine- and carboxybetaine-coated beads. It is unclear whether the aforementioned degradation of the p(CBMAA-2) layers in vacuum or inert atmosphere plays a role in the obtained zeta potential and antifouling properties of this material. 
As can be seen in Figure 4.4, p(HPMAA) performs better than the sulfobetaines, and slightly better than $p($ CBMAA-2) and $p$ (PCMA-2). That $p$ (HPMAA) performs similar or better than $p$ (CBMAA-2) is consistent with literature ${ }^{19,27}$ Why HPMAA and other simple hydroxyl methacrylamide monomers perform so well is not entirely understood. In comparison to zwitterionic brushes, they are only moderately hydrophilic and ionic solvation, as occurs for zwitterionic materials, is stronger than hydrogen-bonding solvation. ${ }^{17,}{ }^{19}$ It might be related to tightly bound water molecules by hydrogen bond bridges that can be formed between one water molecule and the hydroxyl and amide group of the same monomer unit. Preliminary quantum chemical calculations on model systems (B97/6-311+G(d,p) using a PCM selfconsistent reaction field model to simulate water) yielded complexation energies of a HPMAA unit and water - bound by two hydrogen bonds (Figure 4.6) - of 6 to $12 \mathrm{kcal} / \mathrm{mol}$. These data indeed point to strong hydration of HMPAA, and in combination with the antifouling data indicate the usefulness of deeper analyses. Besides the hydration properties, the low surface charge of $\mathrm{p}$ (HPMAA)-coated beads may also contribute to its excellent antifouling properties; p(HPMAA)-coated beads showed the lowest zeta potential of the five monomers tested within this study (see Figure S4.13).

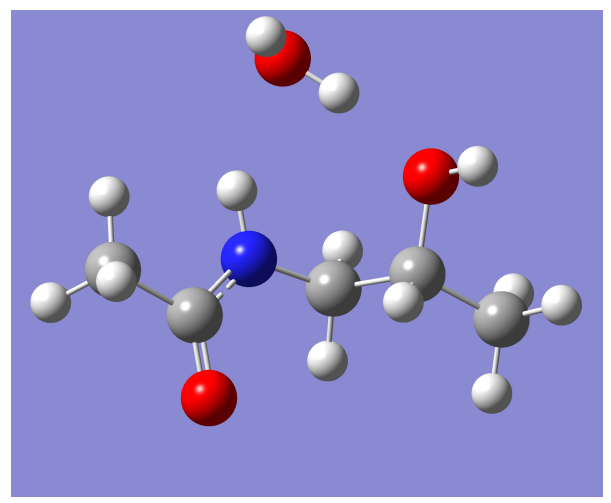

Figure 4.6 Hydration of an HPMAA model via $\mathrm{H}$-bonds consisting of $\mathrm{N}-\mathrm{H} \cdots \mathrm{O}$ and $\mathrm{O}-\mathrm{H} \cdots \mathrm{H}$ interactions (B97D/6-311+G(d,p)-optimized structure; see text for details).

Similar to the hydroxyl acrylamide monomers, phosphocholines are not often compared to other types of antifouling polymer brushes. In a study by Rodriguez-Emmenegger ${ }^{27}$ it was found that $p(\mathrm{PC})$ performed less well than $p(\mathrm{SB}-3)$ and $p(\mathrm{CB}-2)$ - in contrast, we observe that $p$ (PCMA-2) did better than $p$ (SBMAA-3) and equally good as p(CBMAA-2). Noteworthy, the zeta potential of $p$ (PCMA-2) is lower than for the sulfobetaine- and carboxybetaine-coated beads (see Figure S4.13).

Based on our findings, we thus conclude that p(HPMAA)-coated beads are best capable of resisting non-specific protein adsorption from BSA and serum solutions, while all zwitterionic brushes are good, but not as good. In selecting the optimal antifouling coating - besides 
the actual antifouling performance - several other factors come into play, depending on the application. 1) $\mathrm{pH}$ of the medium: the antifouling performance of $\mathrm{p}(\mathrm{CBMAA}-2)$ is $\mathrm{pH}$ dependent $t^{14}$ as the zwitterionic character is lost upon protonation of the carboxylic acid at low pH. 2) Ionic strength and temperature: the antifouling characteristics of sulfobetaines are highly dependent on ionic strength and temperature ${ }^{23-25,51}$ due to strong intra/inter-chain interactions between the sulfobetaine moieties. These are so strong, that without any added ions, sulfobetaine-based polymers can even be used as the basis of self-healing antifouling materials. ${ }^{52,53}$ 3) Biofunctionalization: CBMAA-2 and HPMAA are the only monomers that can directly be functionalized within the brush with bio-recognition elements, ${ }^{2,19}$ albeit at the cost of diminished antifouling properties; ${ }^{54}$ for sulfobetaines an efficient synthesis of azidefunctionalized monomers is available, that allows azide-alkyne click-based biofunctionalizations to take place without loss of the zwitterionic character (see chapter 2, 5 and 6)..$^{33} 4$ ) Ease of use: The growth of sulfobetaine brushes is extremely reproducible and allows for minor deviations from the protocol. Growing HPMAA brushes, on the other hand, turned out to be more challenging, and was found to be more sensitive to oxygen contamination. Further optimization of the synthetic protocols for p(HPMAA) would be well deserved given its antifouling characteristics. PCMA-2 easily self-polymerizes ${ }^{55}$ and was therefore slightly less robust in use than the sulfobetaines. 5) FDA approval: of the materials under study only PCMA2-based brushes are currently FDA approved for biomedical applications. ${ }^{16}$ 6) Characterization: p(CBMAA-2) brush characterization was challenging due to the aforementioned degradation process during UHV analysis. These six factors involved in the use of these different antifouling brushes imply that further research into this field is both required and worthwhile.

\subsection{Conclusion}

In this study we systematically compared five hydrogen-bonding and zwitterionic polymer brushes for their antifouling performance using a bead-based assay. In essence, all brushes fully prevent the non-specific adsorption in single-protein BSA solutions. In solutions containing more complex protein mixtures (e.g. a 10\% serum solution) fouling is reduced by at least an order of magnitude as compared to non-modified beads, but no complete antifouling is observed for any monomer. Our observations lead to the following antifouling ranking based on the amount of adsorbed serum proteins: HPMAA $\geq$ CBMAA-2 $\approx$ PCMA-2 $>$ SBMAA-2 $>$ SBMAA-3 >> non-modified beads.

Each brush has its own advantages and disadvantages, which may direct the preferred use in different situations. Of the family of sulfobetaines, we show for the first time that $p$ (SBMAA-2) performs consistently better in antifouling studies than P(SBMAA-3), due to the reduced spacer length between opposing charges. The excellent performance of p(HPMAA) - equal to or better than any of the zwitterionic monomers under study - is likely related to strong and multiple hydrogen bond formation and/or low surface charge.

We have shown that our bead-based platform is suitable for screening different antifouling coatings for their antifouling capabilities. As thousands of beads can be prepared and analyzed 
at once, it is a valuable and statistically robust method to measure the antifouling behavior of polymer-coated beads in detail.

\subsection{Acknowledgments}

The authors thank Digvijay Gahtory, Judith Firet and Luc Scheres for their help regarding the synthesis of the SBMAA-2 and CBMAA-2 monomer, Andriy Kuzmyn for assistance with the polymerization of HPMAA, René Achterberg for his help using the Biacore SPR apparatus, Remco Fokkink for his input on the zeta potential measurements and Ben Meijer for his assistance with the flow cytometer. This work was supported by NanoNextNL (program 3E), a micro- and nanotechnology consortium of the government of The Netherlands and 130 partners.

\subsection{References}

1. Horbett, T. A. The Role of Adsorbed Proteins in Animal-Cell Adhesion. Colloids Surf., B 1994, 2, 225-240.

2. Jiang, S. Y.; Cao, Z. Q. Ultralow-Fouling, Functionalizable, and Hydrolyzable Zwitterionic Materials and Their Derivatives for Biological Applications. Adv. Mater. 2010, 22, 920-932.

3. Chae, K. H.; Jang, Y. M.; Kim, Y. H.; Sohn, O. J.; Rhee, J. I. Anti-Fouling Epoxy Coatings for Optical Biosensor Application Based on Phosphorylcholine. Sens. Actuators, B 2007, 124, 153-160.

4. Donlan, R. M. Biofilms and Device-Associated Infections. Emerg. Infect. Dis. 2001, 7, 277-281.

5. Chapman, R. G.; Ostuni, E.; Takayama, S.; Holmlin, R. E.; Yan, L.; Whitesides, G. M. Surveying for Surfaces That Resist the Adsorption of Proteins. J.Am. Chem. Soc. 2000, 122, 8303-8304.

6. Ostuni, E.; Chapman, R. G.; Holmlin, R. E.;Takayama, S.; Whitesides, G. M. A Survey of Structure-Property Relationships of Surfaces That Resist the Adsorption of Protein. Langmuir 2001, 17, 5605-5620.

7. Wei, Q.; Becherer,T.; Angioletti-Uberti, S.; Dzubiella, J.; Wischke, C.; Neffe, A. T.; Lendlein, A.; Ballauff, M.; Haag, R. Protein Interactions with Polymer Coatings and Biomaterials. Angew. Chem. Int. Ed. 2014, 53, 8004-8031.

8. Prime, K. L.; Whitesides, G. M. Self-Assembled Organic Monolayers - Model Systems for Studying Adsorption of Proteins at Surfaces. Science 1991, 252, 1164-1167.

9. Li, L. Y.; Chen, S. F.; Zheng, J.; Ratner, B. D.; Jiang, S. Y. Protein Adsorption on Oligo(Ethylene Glycol)-Terminated Alkanethiolate Self-Assembled Monolayers: The Molecular Basis for Nonfouling Behavior. J. Phys. Chem. B 2005, 109, 29342941.

10. Ladd, J.; Zhang, Z.; Chen, S.; Hower, J. C.; Jiang, S. Zwitterionic Polymers Exhibiting High Resistance to Nonspecific Protein Adsorption from Human Serum and Plasma. Biomacromolecules 2008, 9, 1357-1361.

11. Nguyen, A.T.; Baggerman, J.; Paulusse, J. M. J.; van Rijn, C. J. M.; Zuilhof, H. Stable Protein-Repellent Zwitterionic Polymer Brushes Grafted from Silicon Nitride. Langmuir 2011, 27, 2587-2594.

12. Kim, J. C.; Kim, M.; Jung, J.; Kim, H.; Kim, I. J.; Kim, J. R.; Ree, M. Biocompatible Characteristics of Sulfobetaine-Containing Brush Polymers. Macromol. Res. 2012, 20, 746-753.

13. Vaisocherova, H.; Zhang, Z.; Yang, W.; Cao, Z.; Cheng, G.; Taylor, A. D.; Piliarik, M.; Homola, J.; Jiang, S. Functionalizable Surface Platform with Reduced Nonspecific Protein Adsorption from Full Blood Plasma - Material Selection and Protein Immobilization Optimization. Biosens. bioelectron. 2009, 24, 1924-1930.

14. Zhang, Z.; Vaisocherova, H.; Cheng, G.; Yang, W.; Xue, H.; Jiang, S. Y. Nonfouling Behavior of Polycarboxybetaine-Grafted 
Surfaces: Structural and Environmental Effects. Biomacromolecules 2008, 9, 2686-2692.

15. Ning, J. Y.; Li, G.; Haraguchi, K. Synthesis of Highly Stretchable, Mechanically Tough, Zwitterionic Sulfobetaine Nanocomposite Gels with Controlled Thermosensitivities. Macromolecules 2013, 46, 5317-5328.

16. Iwasaki, Y.; Ishihara, K. Cell Membrane-Inspired Phospholipid Polymers for Developing Medical Devices with Excellent Biointerfaces. Sci.Technol.Adv. Mat. 2012, 13, 064101.

17. Chen, H.; Zhao, C.; Zhang, M. Z.; Chen, Q.; Ma, J.; Zheng, J. Molecular Understanding and Structural-Based Design of Polyacrylamides and Polyacrylates as Antifouling Materials. Langmuir 2016, 32, 3315-3330.

18. Pereira, A. D.; Rodriguez-Emmenegger, C.; Surman, F.; Riedel, T.; Alles, A. B.; Brynda, E. Use of Pooled Blood Plasmas in the Assessment of Fouling Resistance. RSC Adv. 2014, 4, 2318-2321.

19. Rodriguez-Emmenegger, C.; Brynda, E.; Riedel, T.; Houska, M.; Subr, V.; Alles, A. B.; Hasan, E.; Gautrot, J. E.; Huck, W.T. S. Polymer Brushes Showing Non-Fouling in Blood Plasma Challenge the Currently Accepted Design of Protein Resistant Surfaces. Macromol. Rapid Commun. 2011, 32, 952-957.

20. Yang, J.; Zhang, M.; Chen, H.; Chang, Y.; Chen, Z.; Zheng, J. Probing the Structural Dependence of Carbon Space Lengths of Poly(N-Hydroxyalkyl Acrylamide)-Based Brushes on Antifouling Performance. Biomacromolecules 2014, 15, $2982-2991$.

21. Vaisocherova, H.; Zhang, Z.; Yang, W.; Cao, Z. Q.; Cheng, G.; Taylor, A. D.; Piliarik, M.; Homola, J.; Jiang, S.Y. Functionalizable Surface Platform with Reduced Nonspecific Protein Adsorption from Full Blood Plasma-Material Selection and Protein Immobilization Optimization. Biosens. Bioelectron. 2009, 24, 1924-1930.

22. Shao, Q.; Jiang, S.Y. Effect of Carbon Spacer Length on Zwitterionic Carboxybetaines. J.Phys. Chem. B 2013, 117, $1357-1366$.

23. Shao, Q.; Jiang, S.Y. Molecular Understanding and Design of Zwitterionic Materials. Adv. Mater. 2015, 27, 15-26.

24. Higaki,Y.; Inutsuka,Y.; Sakamaki,T.; Terayama,Y.; Takenaka, A.; Higaki, K.;Yamada, N. L.; Moriwaki,T.; Ikemoto, Y.; Takahara, A. Effect of Charged Group Spacer Length on Hydration State in Zwitterionic Poly(Sulfobetaine) Brushes. Langmuir 2017, 33, 8404-8412.

25. Wang, T.; Kou, R.; Liu, H.; Liu, L.; Zhang, G.; Liu, G. Anion Specificity of Polyzwitterionic Brushes with Different Carbon Spacer Lengths and Its Application for Controlling Protein Adsorption. Langmuir 2016, 32, 2698-2707.

26. Shao, Q.; Mi, L.; Han, X.; Bai, T.; Liu, S.; Li, Y.; Jiang, S. Differences in Cationic and Anionic Charge Densities Dictate Zwitterionic Associations and Stimuli Responses. J. Phys. Chem. B 2014, 118, 6956-6962.

27. Rodriguez-Emmenegger, C.; Brynda, E.; Riedel, T.; Sedlakova, Z.; Houska, M.; Alles, A. B. Interaction of Blood Plasma with Antifouling Surfaces. Langmuir 2009, 25, 6328-6333.

28. Ueda, T.; Oshida, H.; Kurita, K.; Ishihara, K.; Nakabayashi, N. Preparation of 2-Methacryloyloxyethyl Phosphorylcholine Copolymers with Alkyl Methacrylates and Their Blood Compatibility. Polym.J. 1992, 24, 1259-1269.

29. van Andel, E.; de Bus, I.; Tijhaar, E. J.; Smulders, M. M. J.; Savelkoul, H. F. J.; Zuilhof, H. Highly Specific Binding on Antifouling Zwitterionic Polymer-Coated Microbeads as Measured by Flow Cytometry. ACS Appl. Mater. Interfaces 2017, 9 , 38211-38221.

30. Chen, L.; Honma, Y.; Mizutani, T.; Liaw, D. J.; Gong, J. P.; Osada, Y. Effects of Polyelectrolyte Complexation on the UCST of Zwitterionic Polymer. Polymer 2000, 41, 141-147.

31. Rodriguez-Emmenegger, C.; Schmidt, B. V. K. J.; Sedlakova, Z.; Subr, V.; Alles, A. B.; Brynda, E.; Barner-Kowollik, C. Low Temperature Aqueous Living/Controlled (RAFT) Polymerization of Carboxybetaine Methacrylamide up to High Molecular Weights. Macromol. Rapid Commun. 2011, 32, 958-965.

32. Joshi, S.; Pellacani, P.; van Beek, T. A.; Zuilhof, H.; Nielen, M. W. F. Surface Characterization and Antifouling Properties of Nanostructured Gold Chips for Imaging Surface Plasmon Resonance Biosensing. Sens. Actuators, B 2015, 209, 505-514.

33. Lange, S. C.; van Andel, E.; Smulders, M. M. J.; Zuilhof, H. Efficient and Tunable Three-Dimensional Functionalization of Fully Zwitterionic Antifouling Surface Coatings. Langmuir 2016, 32, 10199-10205.

34. Hofmann, S. Quantitative Analysis (Data Evaluation). In Auger- and X-Ray Photoelectron Spectroscopy in Materials Science; SpringerVerlag: Berlin/Heidelberg, 2013, p 137. 
35. Petrovykh, D. Y.; Kimura-Suda, H.; Tarlov, M. J.; Whitman, L. J. Quantitative Characterization of DNA Films by X-Ray Photoelectron Spectroscopy. Langmuir 2004, 20, 429-440.

36. Gauthier, M.; Carrozzella, T.; Penlidis, A. Sulfobetaine Zwitterionomers Based Onn-Butyl Acrylate and 2-Ethoxyethyl Acrylate: Monomer Synthesis and Copolymerization Behavior. J. Polym. Sci., Part A: Polym. Chem. 2002, 40, 511-523.

37. Ning, J.; Li, G.; Haraguchi, K. Synthesis of Highly Stretchable, Mechanically Tough, Zwitterionic Sulfobetaine Nanocomposite Gels with Controlled Thermosensitivities. Macromolecules 2013, 46, 5317-5328.

38. Tang, Y.; Lu, J. R.; Lewis, A. L.; Vick, T. A.; Stratford, P. W. Swelling of Zwitterionic Polymer Films Characterized by Spectroscopic Ellipsometry. Macromolecules 2001, 34, 8768-8776.

39. Blaszykowski, C.; Sheikh, S.; Thompson, M. A Survey of State-of-the-Art Surface Chemistries to Minimize Fouling from Human and Animal Biofluids. Biomater. Sci. 2015, 3, 1335-1370.

40. Matyjaszewski, K. Atom Transfer Radical Polymerization (ATRP): Current Status and Future Perspectives. Macromolecules 2012, 45, 4015-4039.

41. Yildirim, E.; Cimen, D.; Zengin, A.; Caykara, T. Synthesis of Poly(N-(2-Hydroxypropyl) Methacrylamide) Brushes by Interface-Mediated RAFT Polymerization. RSC Adv. 2016, 6, 45259-45264.

42. Chevalier, Y.; Storet, Y.; Pourchet, S.; Leperchec, P. Tensioactive Properties of Zwitterionic Carboxybetaine Amphiphiles. Langmuir 1991, 7, 848-853.

43. Wielema,T. A.; Engberts, J. B. F. N. Zwitterionic Polymers II Synthesis of a Novel Series of Poly(Vinylbetaines) and the Effect of the Polymeric Structure on the Solubility Behavior in Water. Eur. Polym.J. 1990, 26, 415-421.

44. Cao, B.; Li, L. L.; Tang, Q.; Cheng, G. The Impact of Structure on Elasticity, Switchability, Stability and Functionality of an All-in-One Carboxybetaine Elastomer. Biomaterials 2013, 34, 7592-7600.

45. Shapiro, H. M. Practical Flow Cytometry 4th ed.; WILEY-LISS: Hoboken, New Jersey, 2003.

46. Chen, S. F.; Li, L. Y.; Zhao, C.; Zheng, J. Surface Hydration: Principles and Applications toward Low-Fouling/Nonfouling Biomaterials. Polymer 2010, 51, 5283-5293.

47. Shao, Q.; He, Y.; White, A. D.; Jiang, S.Y. Difference in Hydration between Carboxybetaine and Sulfobetaine. J. Phys. Chem. B 2010, 114, 16625-16631.

48. Jiang, W.; Fischer, G.; Girmay, Y.; Irgum, K. Zwitterionic Stationary Phase with Covalently Bonded Phosphorylcholine Type Polymer Grafts and Its Applicability to Separation of Peptides in the Hydrophilic Interaction Liquid Chromatography Mode. J.Chromatogr., A 2006, 1127, 82-91.

49. Guo, S.; Janczewski, D.; Zhu, X.; Quintana, R.; He,T.; Neoh, K. G. Surface Charge Control for Zwitterionic Polymer Brushes: Tailoring Surface Properties to Antifouling Applications. J. Colloid Interface Sci. 2015, 452, 43-53.

50. Weng, X.-D.; Bao, X.-J.; Jiang, H.-D.; Chen, L.; Ji, Y.-L.; An, Q.-F.; Gao, C.-J. pH-Responsive Nanofiltration Membranes Containing Carboxybetaine with Tunable Ion Selectivity for Charge-Based Separations. J. Membr. Sci. 2016, 520, $294-302$.

51. Gauthier, M.; Carrozzella, T.; Snell, G. Sulfobetaine Zwitterionomers Based on N-Butyl Acrylate and 2-Ethoxyethyl Acrylate: Physical Properties. J. Polym. Sci. Pol. Phys. 2002, 40, 2303-2312.

52. Wang, Z.; van Andel, E.; Pujari, S. P.; Feng, H.; Dijksman, J. A.; Smulders, M. M. J.; Zuilhof, H. Water-Repairable Zwitterionic Polymer Coatings for Anti-Biofouling Surfaces. J. Mater. Chem. B 2017, 5, 6728-6733.

53. Wang, Z.; Fei, G.; Xia, H.; Zuilhof, H. Dual Water-Healable Zwitterionic Polymer Coatings for Anti-Biofouling Surfaces. J. Mater. Chem. B 2018, in press, DOI: 10.1039/c8tb01863d

54. Lisalova, H.; Brynda, E.; Houska, M.; Visova, I.; Mrkvova, K.; Song, X. C.; Gedeonova, E.; Surman, F.; Riedel, T.; PopGeorgievski, O.; Homola, J. Ultralow-Fouling Behavior of Biorecognition Coatings Based on Carboxy-Functional Brushes of Zwitterionic Homo- and Copolymers in Blood Plasma: Functionalization Matters. Anal. Chem. 2017, 89, 3524-3531.

55. Lobb, E. J.; Ma, I.; Billingham, N. C.; Armes, S. P.; Lewis, A. L. Facile Synthesis of Well-Defined, Biocompatible Phosphorylcholine-Based Methacrylate Copolymers Via Atom Transfer Radical Polymerization at 20 Degrees C. J.Am. Chem. Soc. 2001, 123, 7913-7914. 


\subsection{Supporting Information}

\subsubsection{Characterization of synthesized monomers}

\section{3-((3-METHACRYLAMIDOPROPYL)DIMETHYLAMMONIO)ETHANE-1- SULFONATE (SBMAA-2)}

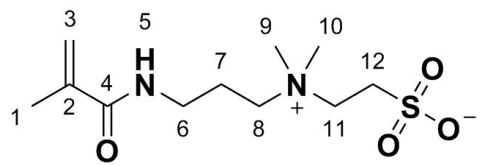

Scheme S4.1 SBMAA-2 structure.

Yield: $4.5 \mathrm{~g}(16 \mathrm{mmol}, 56 \%),\left[\mathrm{C}_{11} \mathrm{H}_{22} \mathrm{~N}_{2} \mathrm{O}_{4} \mathrm{~S}, \mathrm{M}=278.37 \mathrm{~g} / \mathrm{mol}\right]$

${ }^{1} \mathrm{H}$ NMR (400 MHz, D O) $\delta 5.62$ (s, 1H, 3a), 5.37 (s, 1H, 3b), $3.65-3.55(\mathrm{~m}, 2 \mathrm{H}, 11-\mathrm{H})$, $3.34-3.23(\mathrm{~m}, 6 \mathrm{H}, 6,8,12-\mathrm{H}), 3.05(\mathrm{~s}, 6 \mathrm{H}, 9,10-\mathrm{H}), 1.97(\mathrm{dt}, \mathrm{J}=14.9,6.7 \mathrm{~Hz}, 2 \mathrm{H}, 7-\mathrm{H})$, $1.83(\mathrm{~s}, 3 \mathrm{H}, 1 \mathrm{H}) \mathrm{ppm}$.

${ }^{13} \mathrm{C}$ NMR (101 MHz, D O) $\delta 171.99$ (C-4), 138.82 (C-2), 121.36 (C-3), 62.20 (C-8), 59.17 (C-11), 50.93 (C-9,10), 44.05 (C-6), 36.13 (C-12), 22.28 (C-7), 17.60 (C-1) ppm.

ESI $^{+}(\mathrm{MeOH}):$ calc.: $\mathrm{m} / \mathrm{z}=301.1198[\mathrm{M}+\mathrm{Na}]^{+}$; det.: $\mathrm{m} / \mathrm{z}=301.1187[\mathrm{M}+\mathrm{Na}]^{+}$.

IR (ATR): $v=3382\left(\mathrm{w}, \mathrm{N}-\mathrm{H}\right.$ stretching), 3041 (w, asymmetric $\mathrm{CH}_{2}$ stretching), 2947 (w, asymmetric $\mathrm{CH}_{2}$ stretching), 1656 (m, C=O bending), 1614 (m, alkene stretching), 1524 (m, $\mathrm{N}-\mathrm{H}$ bending), 1202 (s, $\mathrm{SO}_{2}$ symmetric stretching), 1038 (s, $\mathrm{SO}_{3}$ stretching) $\mathrm{cm}^{-1}$.

\section{3-((3-METHACRYLAMIDOPROPYL)DIMETHYLAMMONIO)PROPANE-1-} SULFONATE (SBMAA-3)

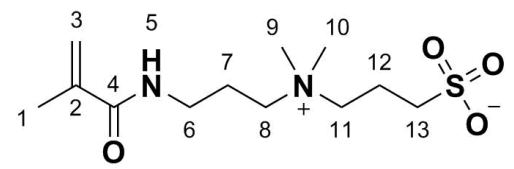

Scheme S4.2 SBMAA-3 structure.

Yield: $15.1 \mathrm{~g}(51.7 \mathrm{mmol}, 94 \%),\left[\mathrm{C}_{12} \mathrm{H}_{24} \mathrm{~N}_{2} \mathrm{O}_{4} \mathrm{~S}, \mathrm{M}=292.39 \mathrm{~g} / \mathrm{mol}\right]$

${ }^{1} \mathrm{H}$ NMR $\left(400 \mathrm{MHz}, \mathrm{D}_{2} \mathrm{O}\right) \delta 5.68(\mathrm{~s}, 1 \mathrm{H}, 3 \mathrm{a}-\mathrm{H}), 5.44(\mathrm{~s}, 1 \mathrm{H}, 3 \mathrm{~b}-\mathrm{H}), 3.49-3.40(\mathrm{~m}, 2 \mathrm{H}, 11-$ 
H), $3.38-3.29(\mathrm{~m}, 4 \mathrm{H}, 6,8-\mathrm{H}), 3.08$ (s, 6H, 9,10-H), $2.94(\mathrm{t}, \mathrm{J}=7.2 \mathrm{~Hz}, 2 \mathrm{H}, 13-\mathrm{H}), 2.24$ -2.11 (m, 2H, 12-H), 2.07-1.96 (m, 2H, 7-H), 1.90 (s, 3H, 1-H) ppm.

${ }^{13} \mathrm{C}$ NMR (101 MHz, D $\left.\mathrm{O}\right) \delta 171.95$ (C4), 138.76 (C2), 121.33 (C3), 62.12 (C11), 61.81 (C8), 50.68 (C9,10), 47.14 (C13), 36.15 (C6), 22.21 (C7), 18.08 (C12), 17.57 (C1) ppm.

ESI $^{+}(\mathrm{MeOH}):$ calc.: $\mathrm{m} / \mathrm{z}=293.1530[\mathrm{M}+\mathrm{H}]^{+}, 315.1349[\mathrm{M}+\mathrm{Na}]^{+}, 585.2986[2 \mathrm{M}+\mathrm{H}]^{+}$, $607.2806[2 \mathrm{M}+\mathrm{Na}]^{+} ;$det.: $\mathrm{m} / \mathrm{z}=293.1515[\mathrm{M}+\mathrm{H}]^{+}, 315.1332[\mathrm{M}+\mathrm{Na}]^{+}$, $585.2956[2 \mathrm{M}+\mathrm{H}]^{+}, 607.2774[2 \mathrm{M}+\mathrm{Na}]^{+}$.

IR (ATR): $v=3305$ (w, N-H stretching), 3031 (w, asymmetric $\mathrm{CH}_{2}$ stretching), 2977 (w, symmetric $\mathrm{CH}_{2}$ stretching), 1656 (m, C=O bending), 1614 (m, alkene stretching), 1546 (m, $\mathrm{N}-\mathrm{H}$ bending), 11911174 (s, $\mathrm{SO}_{2}$ symmetric stretching ), 1038 (s, $\mathrm{SO}_{3}$ stretching ) $\mathrm{cm}^{-1}$.

\section{3-((3-METHACRYLAMIDOPROPYL)DIMETHYLAMMONIO)PROPANOATE (CB- MAA-2)}

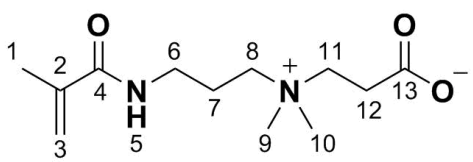

Scheme $\mathbf{S 4 . 3}$ CBMAA-2 structure.

Yield: $30.0 \mathrm{~g}(124 \mathrm{mmol}, 45 \%),\left[\mathrm{C}_{12} \mathrm{H}_{22} \mathrm{~N}_{2} \mathrm{O}_{3}, \mathrm{M}=242.32 \mathrm{~g} / \mathrm{mol}\right]$

${ }^{1} \mathrm{H}$ NMR $\left(400 \mathrm{MHz}, \mathrm{D}_{2} \mathrm{O}\right) \delta 5.63(\mathrm{~s}, 1 \mathrm{H}, 3 \mathrm{a}-\mathrm{H}), 5.39(\mathrm{~s}, 1 \mathrm{H}, 3 \mathrm{~b}-\mathrm{H}), 3.48(\mathrm{t}, \mathrm{J}=7.8 \mathrm{~Hz}, 2 \mathrm{H}$, 6-H), $3.32-3.20(\mathrm{~m}, 4 \mathrm{H}, 8,11-\mathrm{H}), 2.99$ (s, 6H, 9,10-H), $2.62-2.52$ (m, 2H, 12-H), 2.03 $-1.90(\mathrm{~m}, 2 \mathrm{H}, 7-\mathrm{H}), 1.84$ (d, J = $1.2 \mathrm{~Hz}, 3 \mathrm{H}, 1-\mathrm{H}) \mathrm{ppm}$.

${ }^{13} \mathrm{C}$ NMR (101 MHz, D O) $\delta 176.53$ (C-13), 172.03 (C-4), 138.81 (C-2), 121.29 (C-3), 61.93 (C-11), 61.19 (C-8), 50.55-50.46 (C-9,10), 36.19 (C-6), 30.71 (C-12), 22.22 (C-7), $17.56(\mathrm{C}-1) \mathrm{ppm}$.

ESI $^{+}(\mathrm{MeOH}):$ calc.: $\mathrm{m} / \mathrm{z}=243.1703[\mathrm{M}+\mathrm{H}]^{+}, 265.1523[\mathrm{M}+\mathrm{Na}]^{+}$; det: $\mathrm{m} / \mathrm{z}=243.1698$ $[\mathrm{M}+\mathrm{H}]^{+}, 265.1517[\mathrm{M}+\mathrm{Na}]^{+}$.

IR (ATR): $v=3284\left(\mathrm{~m}\right.$, carboxylate), 3037 (w, asymmetric $\mathrm{CH}_{2}$ stretching), 2957 (w, symmetric $\mathrm{CH}_{2}$ stretching), 1734 (w, carboxylic acid $\mathrm{C}=\mathrm{O}$ bending), 1658 (m, amide $\mathrm{C}=\mathrm{O}$ bending), 1591 (s, alkene stretching), 1540 (s, N-H bending) $\mathrm{cm}^{-1}$. 


\subsubsection{Additional XPS spectra}
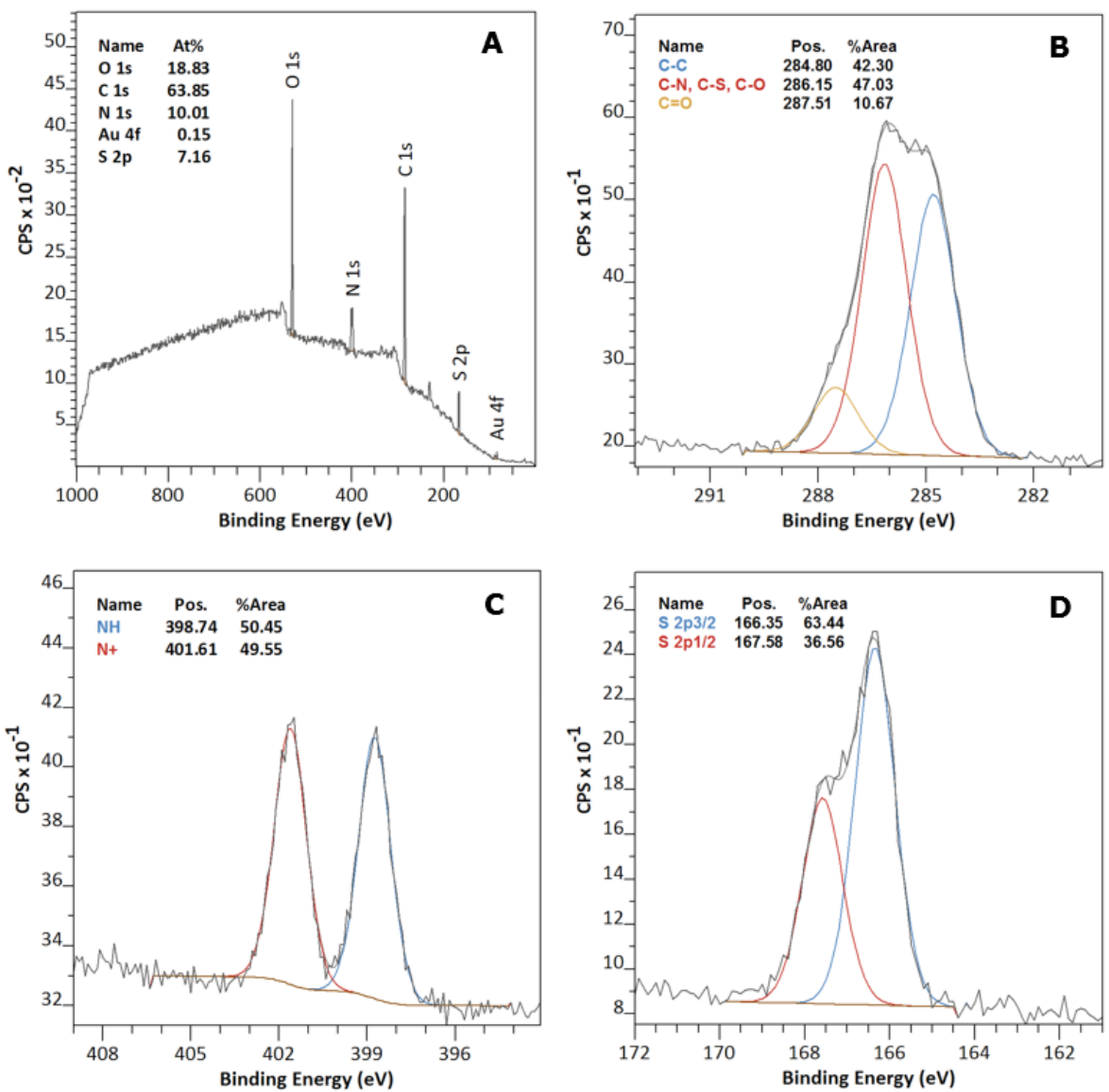

Figure S4.1 XPS spectra of gold surfaces coated with SBMAA-2 polymer brushes grown via SI-ATRP: (A) wide scan spectrum, (B) C 1s narrow scan spectrum, (C) N 1s narrow scan spectrum and (D) S 2p narrow scan spectrum. 

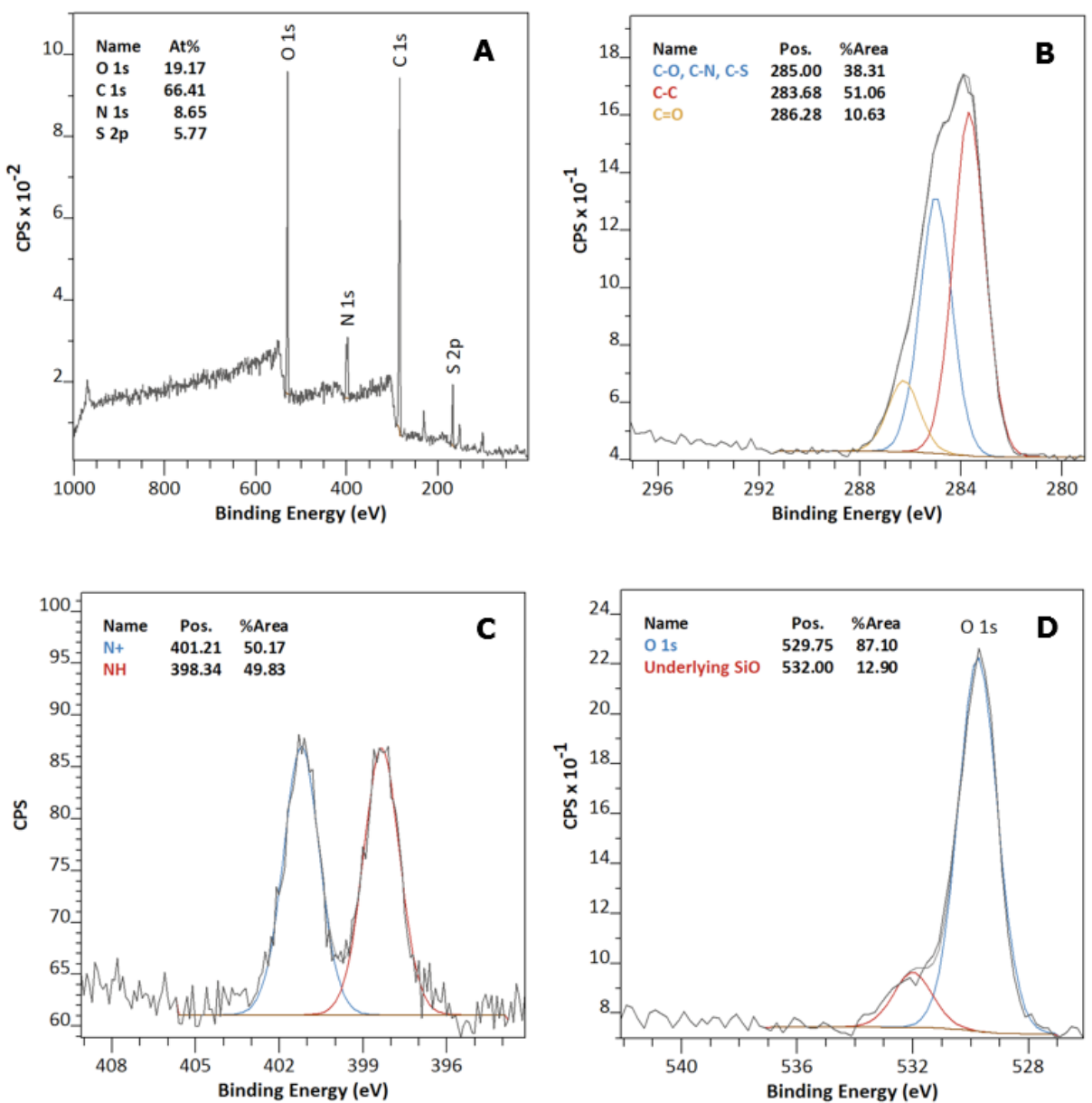

Figure S4.2 XPS spectra of Dynabeads modified with SBMAA-2 polymer brushes grown via SI-ATRP: (A) wide scan spectrum, (B) C 1s narrow scan spectrum, (C) N 1s narrow scan spectrum and (D) O 1s narrow scan spectrum. SBMAA-2-coated Dynabeads were dropcasted on a $\mathrm{Si}(111)$ surface to allow for XPS analysis. *The two extra peaks (to the right of the S 2p peak) in the wide scans and the minor oxygen peak in thw oxygen narrow scan spectrum originate from the underlying $\mathrm{SiO}$ layer. 

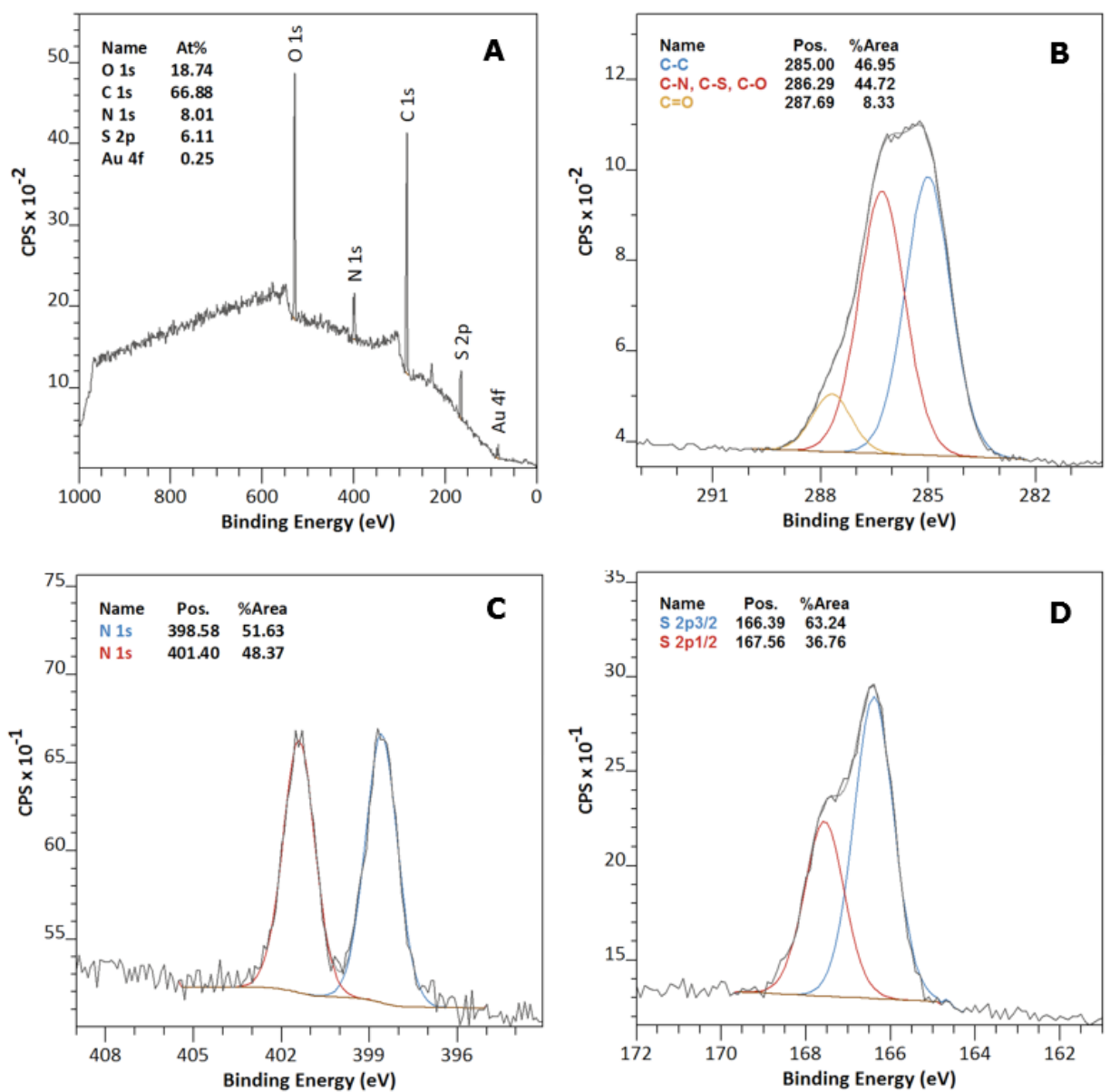

Figure S4.3 XPS spectra of gold surfaces coated with SBMAA-3 polymer brushes grown via SI-ATRP: (A) wide scan spectrum, (B) C 1s narrow scan spectrum, (C) $\mathrm{N}$ 1s narrow scan spectrum and (D) S 2p narrow scan spectrum. 

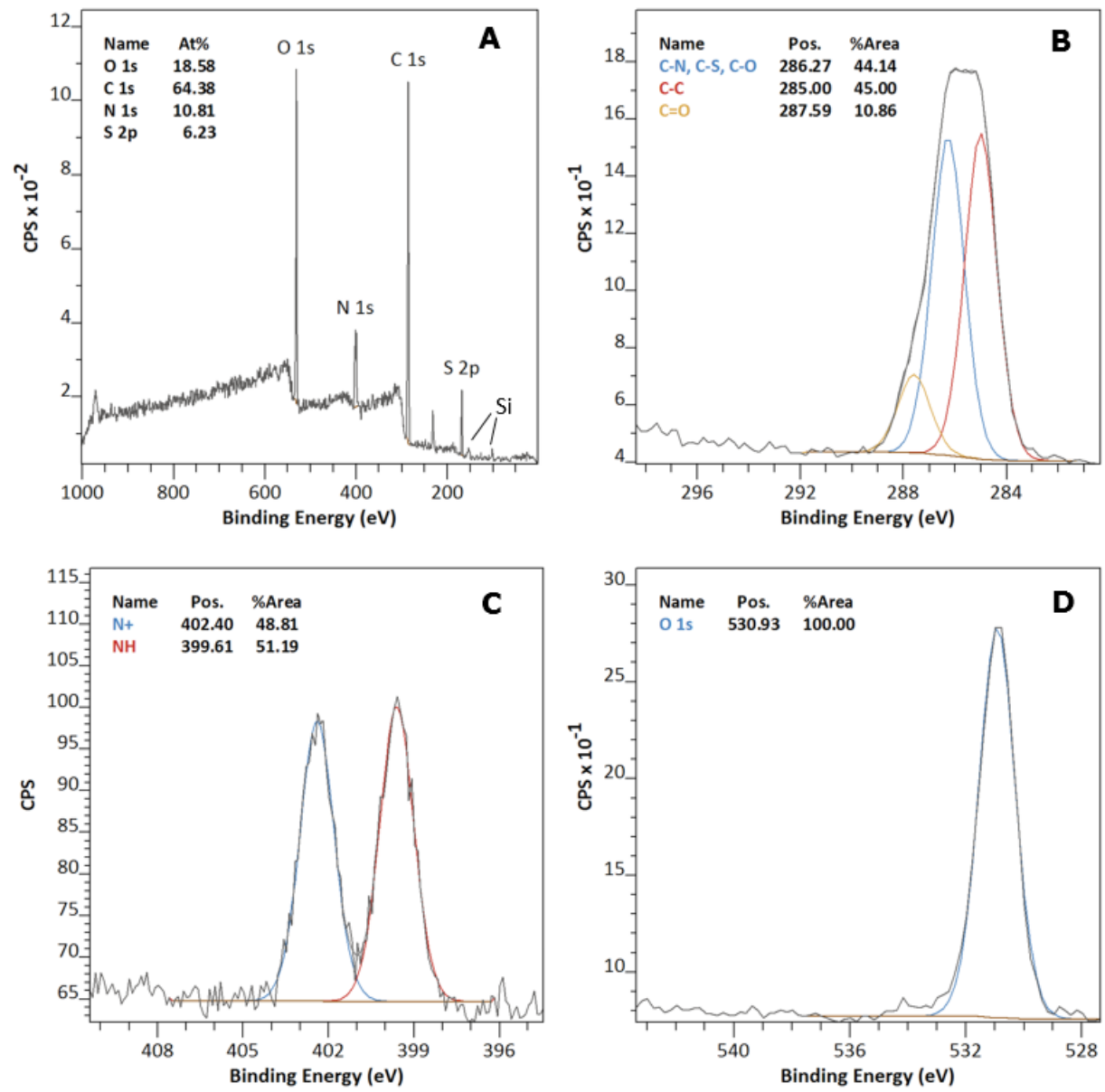

Figure S4.4 XPS spectra of Dynabeads modified with SBMAA-3 polymer brushes grown via SI-ATRP: (A) wide scan spectrum, (B) C 1s narrow scan spectrum, (C) $\mathrm{N}$ 1s narrow scan spectrum and (D) $\mathrm{O} 1 \mathrm{~s}$ narrow scan spectrum. SBMAA-3-coated Dynabeads were dropcasted on a $\mathrm{Si}(111)$ surface to allow for XPS analysis (hence the small Si peaks in the wide scan spectrum). 

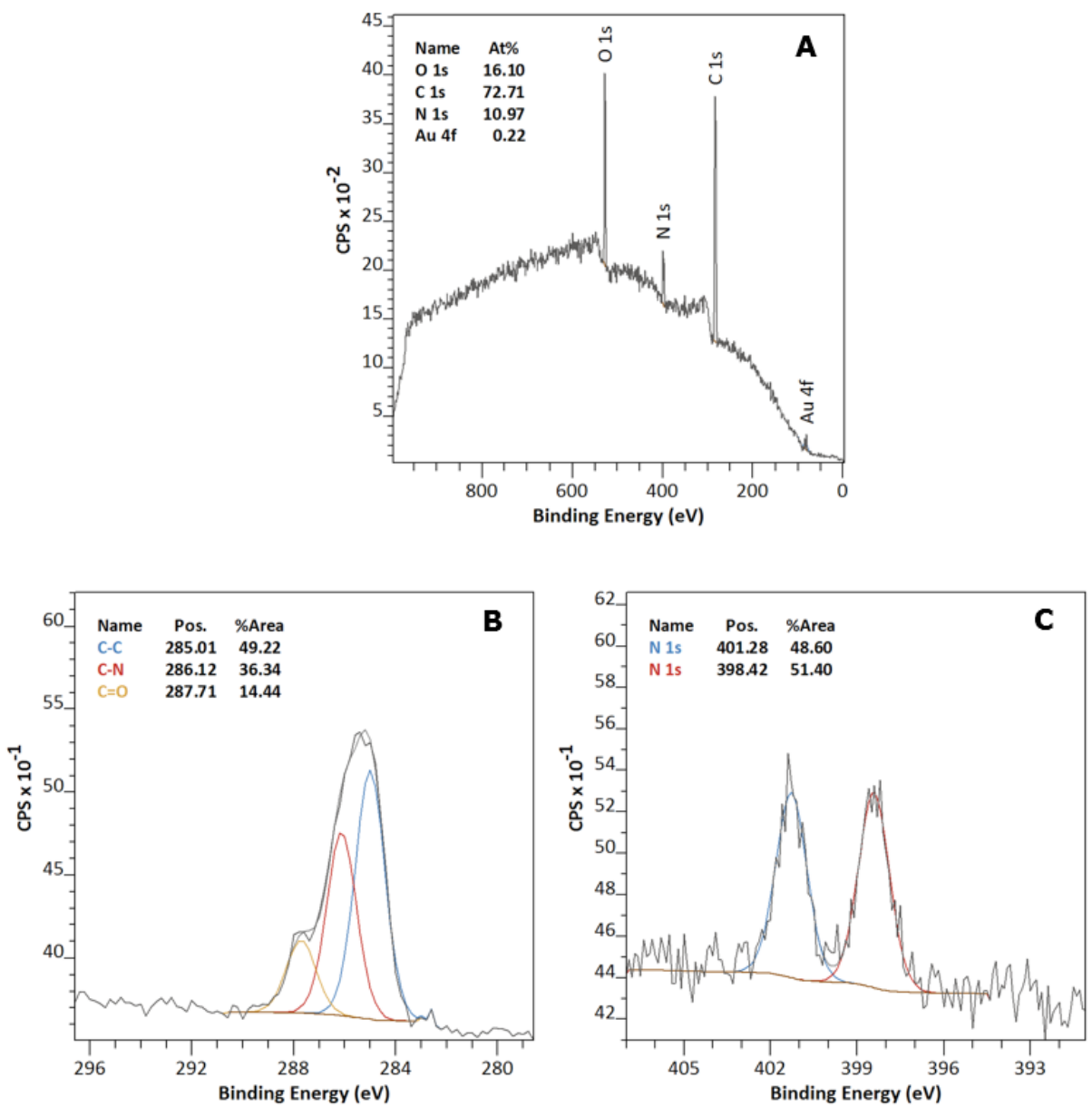

Figure S4.5 XPS spectra of gold surfaces coated with CBMAA-2 polymer brushes grown via SI-ATRP: (A) wide scan spectrum and (B) N 1s narrow scan spectrum. 

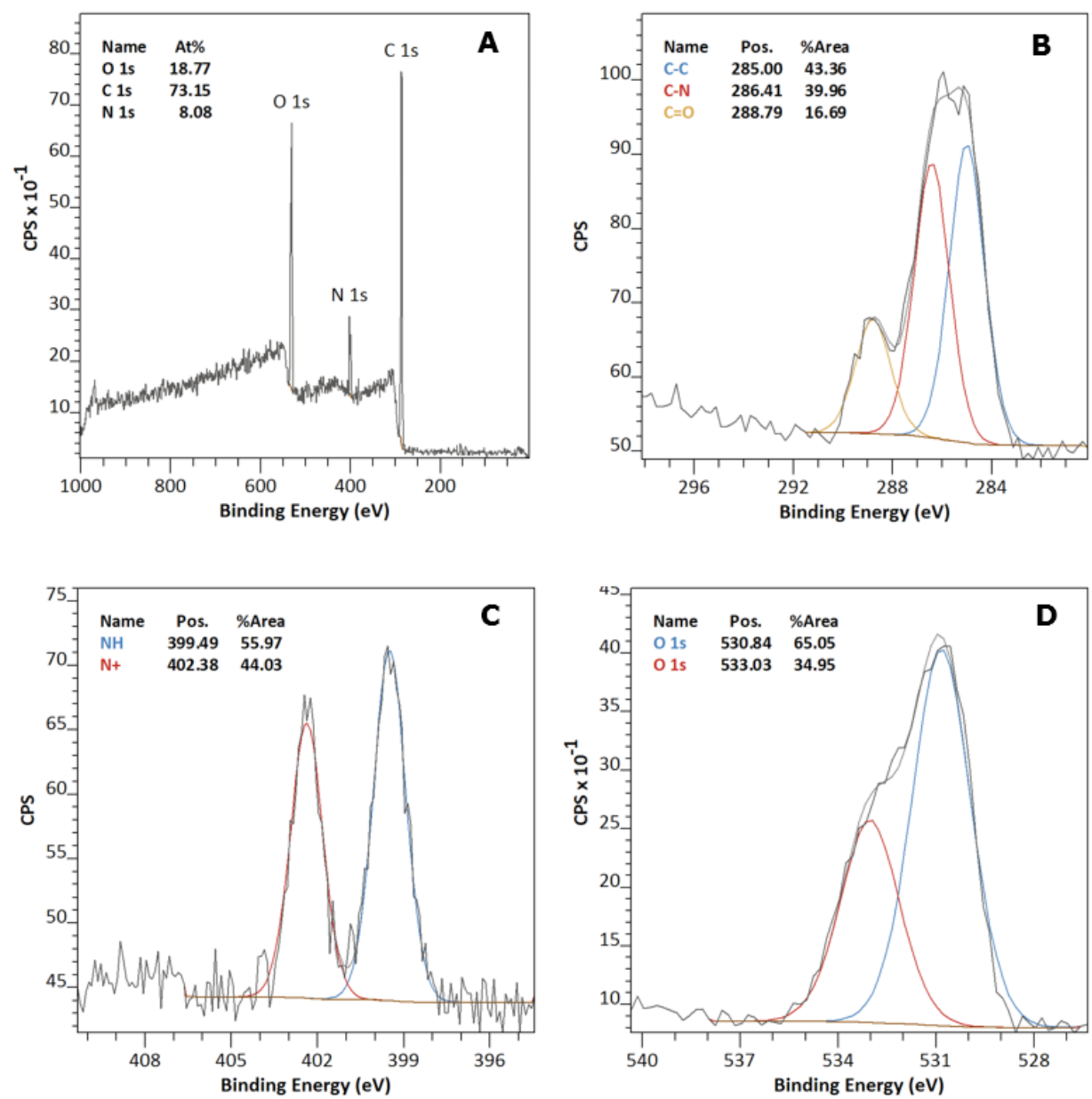

Figure S4.6 XPS spectra of Dynabeads modified with CBMAA-2 polymer brushes grown via SI-ATRP: (A) wide scan spectrum, (B) C 1s narrow scan spectrum, (C) N 1s narrow scan spectrum and (D) O 1s narrow scan spectrum. CBMAA-2-coated Dynabeads were dropcasted on a $\mathrm{Si}(111)$ surface to allow for XPS analysis. 

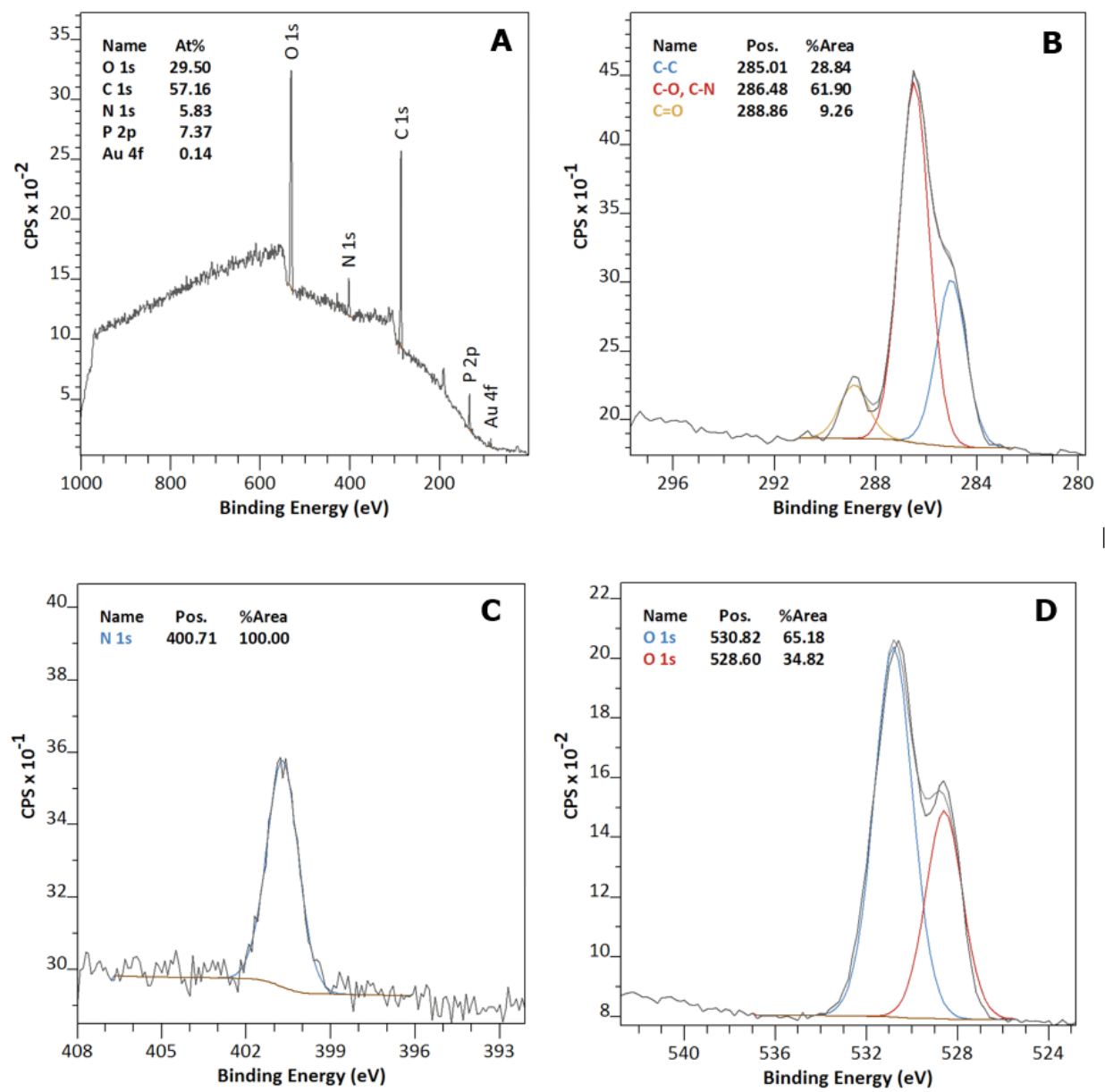

Figure S4.7 XPS spectra of gold surfaces coated with PCMA-2 polymer brushes grown via SI-ATRP: (A) wide scan spectrum, (B) C 1s narrow scan spectrum, (C) N 1s narrow scan spectrum and (D) O 1s narrow scan spectrum. 

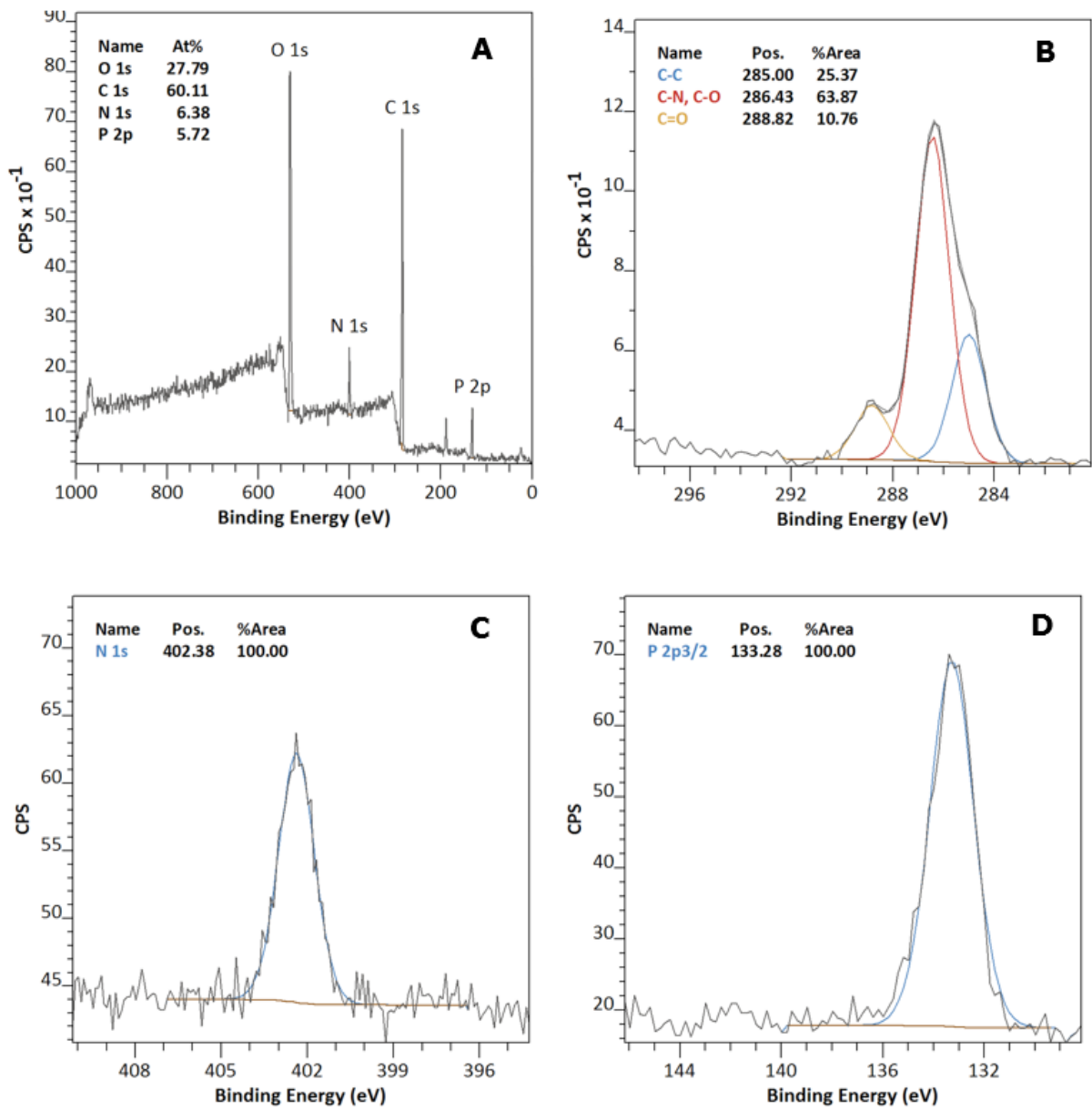

Figure S4.8 XPS spectra of Dynabeads modified with PCMA-2 polymer brushes grown via SI-ATRP: (A) wide scan spectrum, (B) C 1s narrow scan spectrum, (C) $\mathrm{N}$ 1s narrow scan spectrum and (D) P 2p narrow scan spectrum. PCMA-2-coated Dynabeads were dropcasted on a Si(111) surface to allow for XPS analysis. 

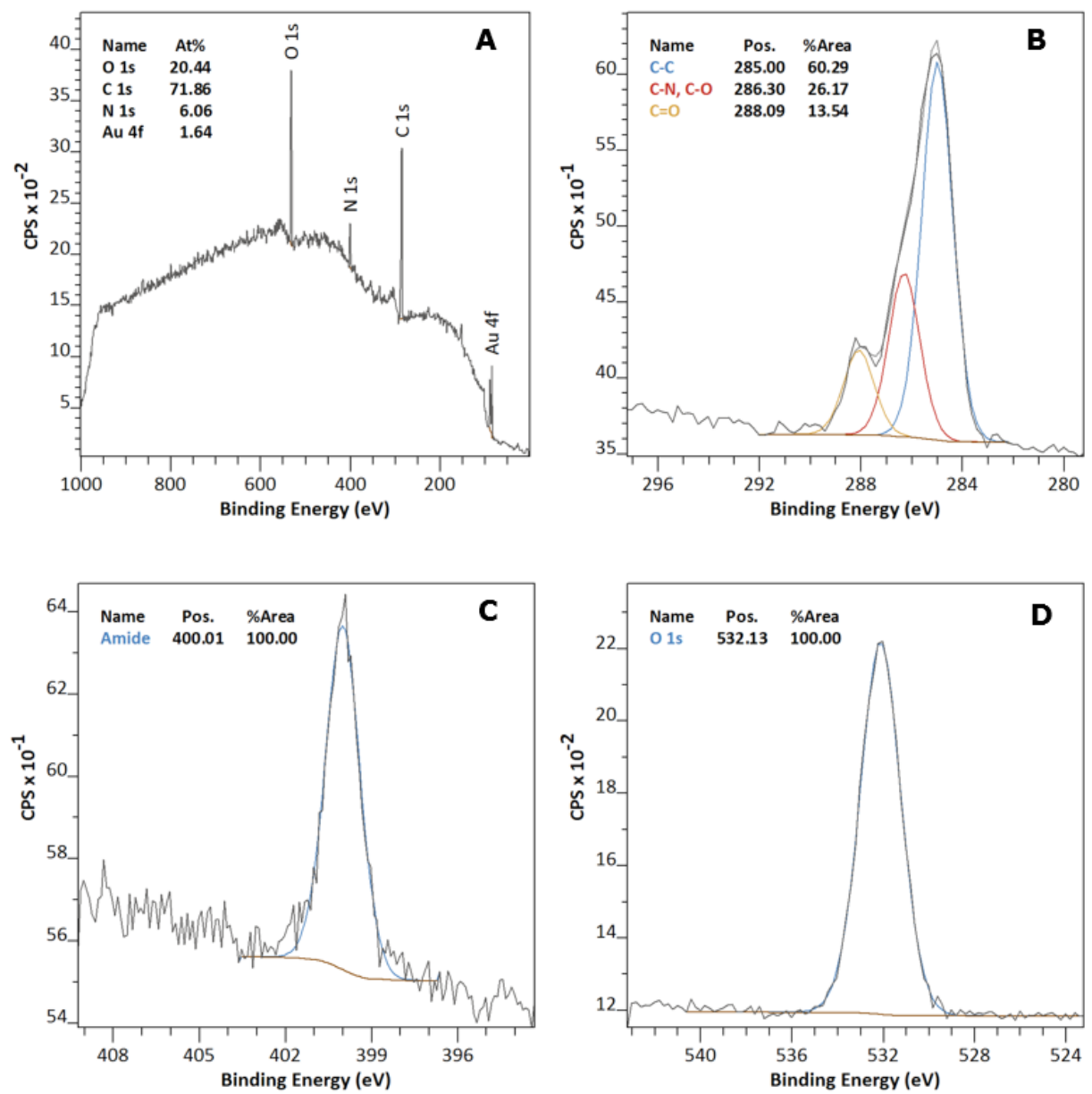

Figure S4.9 XPS spectra of gold surfaces coated with HPMAA polymer brushes grown via SI-ATRP: (A) wide scan spectrum, (B) C 1s narrow scan spectrum, (C) N 1s narrow scan spectrum and (D) O 1s narrow scan spectrum. 

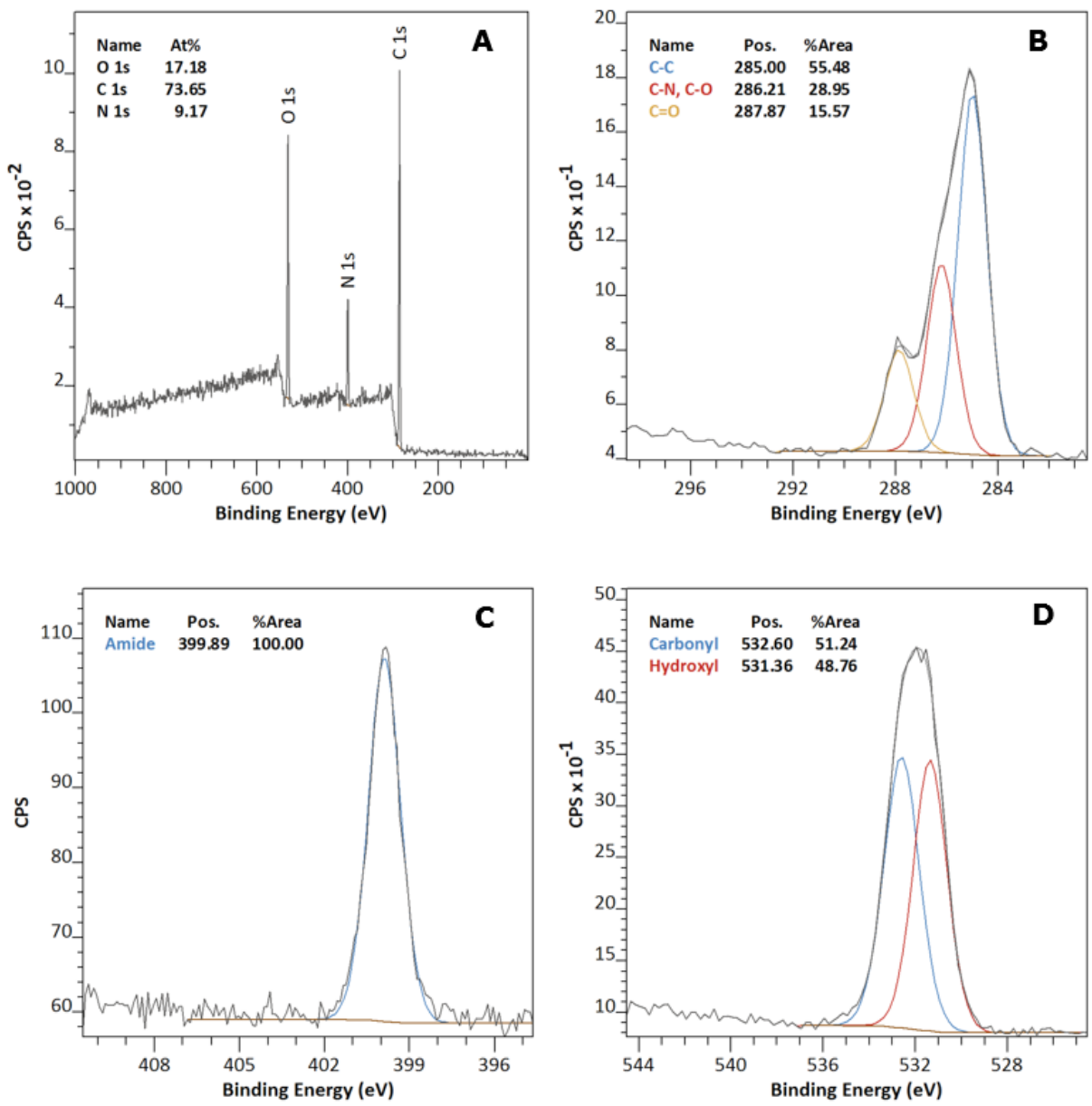

Figure S4.10 XPS spectra of Dynabeads modified with HPMAA polymer brushes grown via SI-ATRP: (A) wide scan spectrum, (B) C 1s narrow scan spectrum, (C) N 1s narrow scan spectrum and (D) O 1s narrow scan spectrum. p(HPMAA)-coated Dynabeads were dropcasted on a Si(111) surface to allow for XPS analysis. 

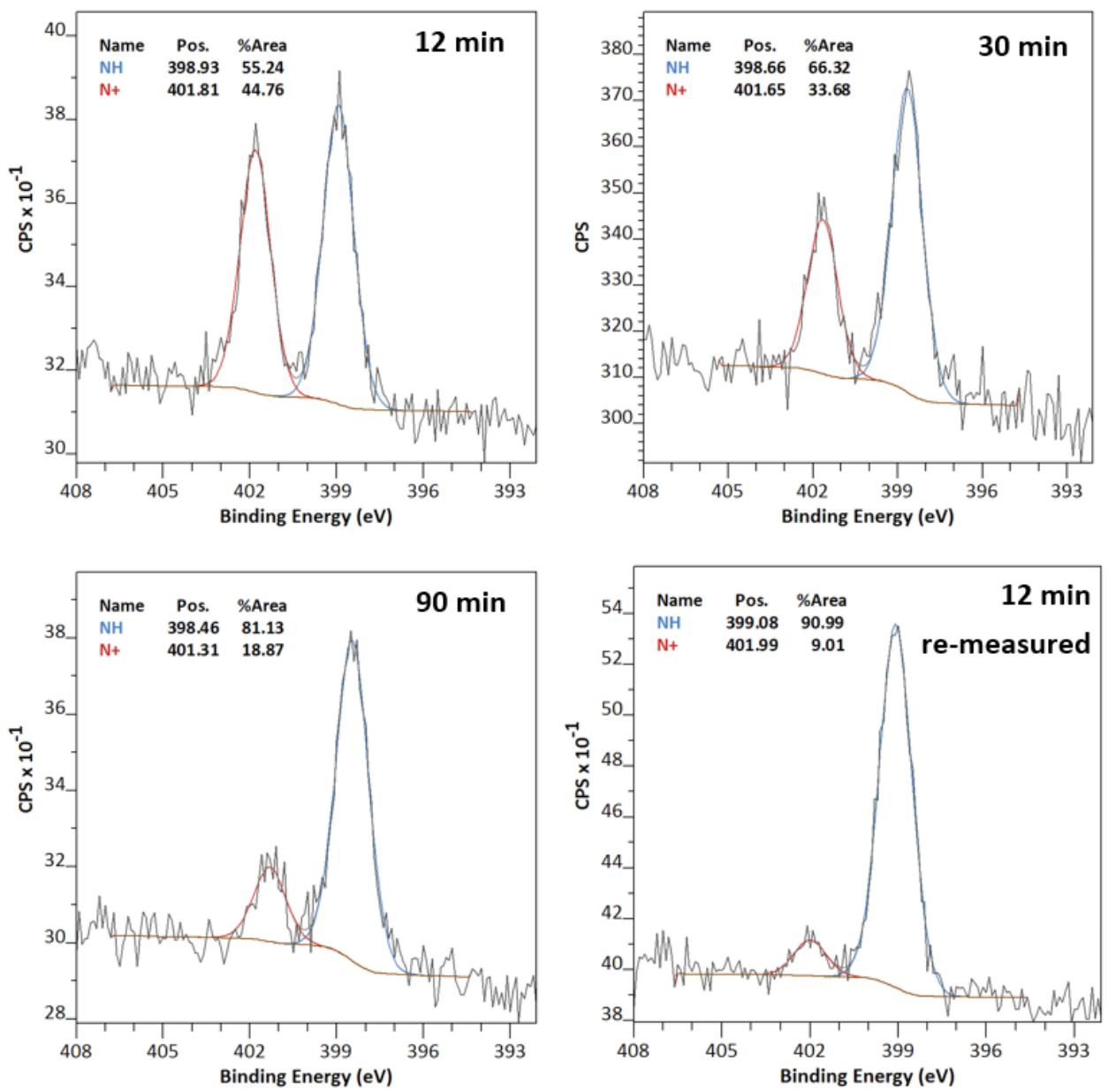

Figure S4.11 XPS N 1s narrow scan spectra of P(CBMAA-2)-coated gold surfaces grown via SI-ATRP for 12, 30 and $90 \mathrm{~min}$. The surfaces were placed together inside the XPS chamber and measured sequentially in the order of 12, 30 and 90 min ATRP. Without having taken the samples out of the XPS, the 12 min gold surface was measured again. These data revealed that the time within the XPS chamber affects the relative heights of the $\mathrm{N} 1 \mathrm{~s}$ peaks. 

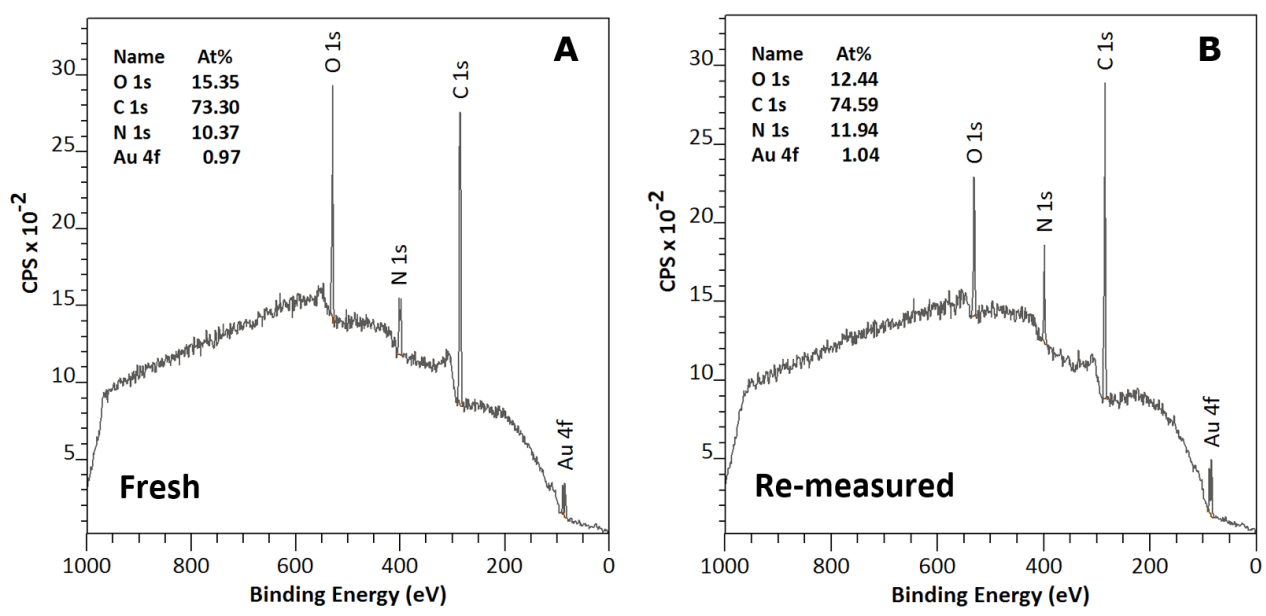

Figure S4.12 XPS wide scan spectra of a p(CBMAA-2)-coated gold surface prepared by 12 min SI-ATRP: either measured directly (A) or after approximately 12 hours (B) without having taken the sample out of the XPS.

\subsubsection{Zeta potential measurements}

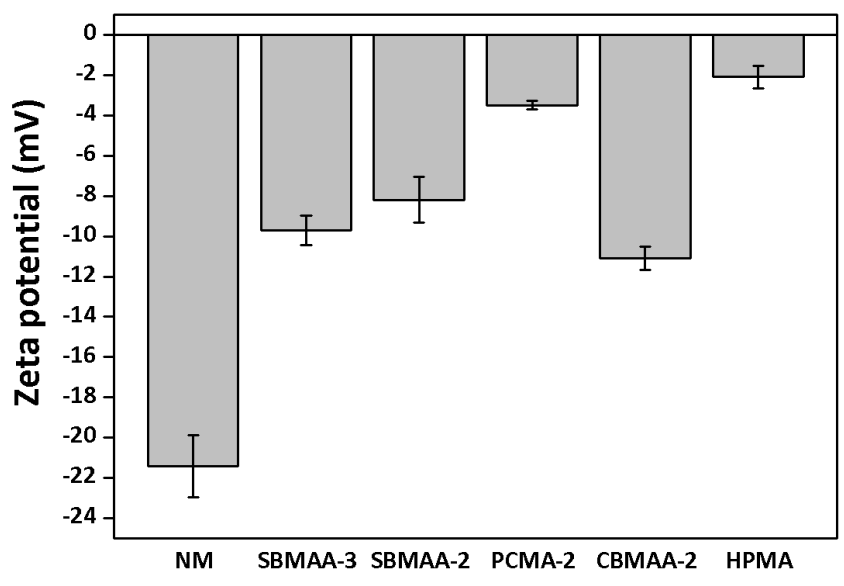

Figure S4.13 Zeta potentials of non-modified (NM) and polymer-coated Dynabeads, measured in PBS $\mathrm{pH} 7.4$ at $25^{\circ} \mathrm{C}$. Presented data are averages of two independent measurements, standard deviations are presented as error bars. ${ }^{*} \mathrm{NM}$ beads showed positive zeta potentials in MilliQ (30.6 \pm 0.7$)$ whereas all polymer-coated beads were negative in MilliQ. 


\subsubsection{Additional flow cytometry data}

\begin{tabular}{l|l|l|l|l|l} 
Sample & PBS & BSA & $\begin{array}{l}\text { Corrected } \\
\text { value }\end{array}$ & Average & $\begin{array}{l}\text { Standard } \\
\text { deviation }\end{array}$ \\
\hline Non-modified & 208 & 2409 & 2201 & & \\
& 163 & 1967 & 1804 & & \\
& 160 & 2782 & 2622 & 2209.0 & 409.1 \\
\hline SBMAA-3 & 312 & 321 & 9 & & \\
& 253 & 258 & 5 & 7.0 & 2.8 \\
\hline SBMAA-2 & 318 & 336 & 18 & & \\
& 256 & 273 & 17 & 17.5 & 0.7 \\
\hline CBMAA-2 & 210 & 221 & 11 & & \\
& 229 & 246 & 17 & 14.0 & 4.2 \\
\hline PCMA-2 & 310 & 311 & 1 & & \\
& 244 & 257 & 13 & 7.0 & 8.5 \\
\hline HPMAA & 311 & 318 & 7 & & \\
& 253 & 261 & 8 & 7.5 & 0.7
\end{tabular}

Table S4.1 Median fluorescence data (FITC-channel) obtained for non-modified and polymer-coated beads incubated in PBS or BSA-AF488 $(0.5 \mathrm{mg} / \mathrm{mL}$ in PBS). PBS values are subtracted from BSA values to obtain the corrected values. The listed averages and standard deviations are used for Figure 4.4.

\begin{tabular}{l|l|l|l|l|l} 
Sample & PBS & Serum & $\begin{array}{l}\text { Corrected } \\
\text { value }\end{array}$ & Average & $\begin{array}{l}\text { Standard } \\
\text { deviation }\end{array}$ \\
\hline Non-modified & 95.7 & 8980 & 8884.3 & & \\
& 97.5 & 9915 & 9817.5 & & \\
& 95.1 & 8430 & 8334.9 & 9012.2 & 749.5 \\
\hline SBMAA-3 & 121 & 644 & 523 & & \\
& 135 & 655 & 520 & 521.5 & 2.1 \\
\hline SBMAA-2 & 130 & 344 & 214 & & \\
& 121 & 266 & 145 & & \\
& 135 & 332 & 197 & 185.3 & 35.9 \\
\hline CBMAA-2 & 115 & 142 & 27 & & \\
& 121 & 205 & 84 & 55.5 & 40.3 \\
\hline PCMA-2 & 129 & 171 & 42 & & \\
& 121 & 185 & 64 & & \\
& 133 & 226 & 93 & 66.3 & 25.6 \\
\hline HPMAA & 129 & 155 & 26 & & \\
& 121 & 154 & 33 & & \\
& 134 & 181 & 47 & 35.3 & 10.7
\end{tabular}

Table S4.2 Median fluorescence data (PE-channel) obtained for non-modified and polymer-coated beads incubated in PBS or biotinylated serum $(\sim 6 \mathrm{mg} / \mathrm{mL})$ followed by Strep-PE. PBS values are subtracted from values for serum to obtain the corrected values. The listed averages and standard deviations are used for Figure 4.4 . 


\subsubsection{SPR experiments}

During our SPR experiments using sulfobetaine-coated surfaces, we observed a negative dip in the relative response units just after sample injection. The signal only stabilized after an extended time of flushing with running buffer. The dip was attributed to the difference in salt concentration between running buffer and sample. This is supported by a similar dip that was observed when we injected buffer with an increased salt concentration. No dip was observed when a sucrose solution was injected with equal salt concentration between sample and running buffer. The dip is probably caused by a sudden swelling of the brushes caused by a difference in salt concentration between serum and the running buffer. Sulfobetaine-based materials swell considerably with increased ionic strength ${ }^{\mathrm{S1}, \mathrm{s} 2}$ and as the refractive index lowers upon swelling, ${ }^{\mathrm{S3}}$ a decrease in signal can be observed. To avoid salt effects, $100 \%$ bovine serum (pooled from 3 adult cows) was dialyzed against the running buffer. The serum dilutions were prepared from the dialyzed serum solution. Long injection times and low flow rates were needed in order to get enough fouling to be able to discriminate SBMAA-3 from SBMAA-2.

\subsection{References}

S1. Higaki,Y.; Inutsuka, Y.; Sakamaki,T.; Terayama,Y.; Takenaka, A.; Higaki, K.; Yamada, N. L.; Moriwaki, T.; Ikemoto, Y.; Takahara, A. Effect of Charged Group Spacer Length on Hydration State in Zwitterionic Poly(sulfobetaine) Brushes. Langmuir 2017, 33, 8404-8412.

S2. Wang, T.; Kou, R.; Liu, H.; Liu, L.; Zhang, G.; Liu, G. Anion Specificity of Polyzwitterionic Brushes with Different Carbon Spacer Lengths and Its Application for Controlling Protein Adsorption. Langmuir 2016, 32, 2698-2707.

S3. Tang,Y.; Lu, J. R.; Lewis, A. L.;Vick,T.A.; Stratford, P.W. Swelling of zwitterionic polymer films characterized by spectroscopic ellipsometry. Macromolecules 2001, 34, 8768-8776. 


\section{Chapter.}

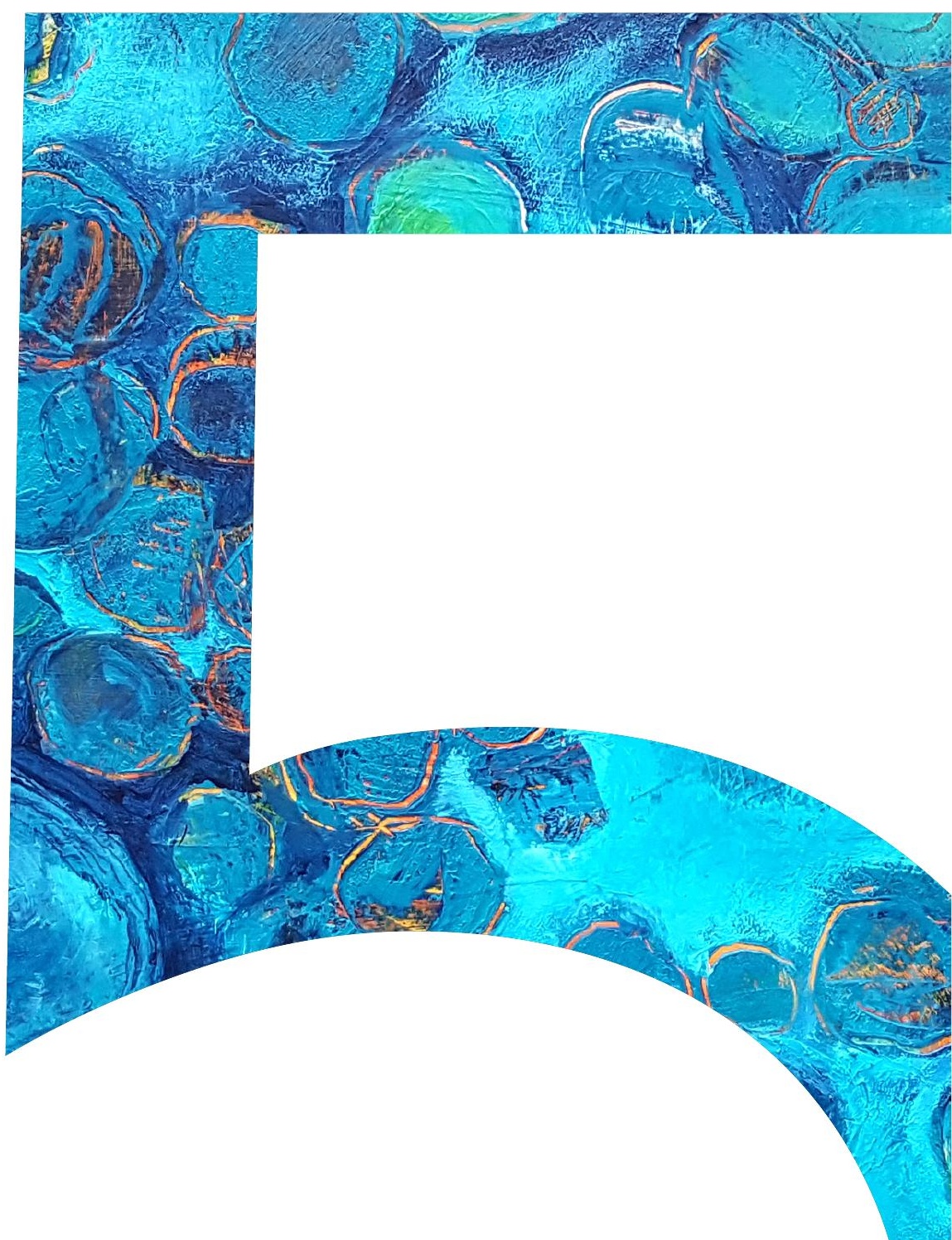




\section{Specific Protein Identification by Immunoprecipitation- Mass Spectrometry Using Antifouling Microbeads}

van Andel, E.; Roosjen, M.; van der Zanden, S.; Lange, S. C.; Weijers, D.; Smulders, M. M. J.; Savelkoul, H. F. J.; Zuilhof, H.; Tijhaar, E. J. Specific Protein Identification by ImmunoprecipitationMass Spectrometry Using Antifouling Microbeads. 2018, Manuscript in preparation. 


\begin{abstract}
Proteins play an essential role in many biological processes, typically acting in multiprotein complexes. To fully understand how proteins function within these complexes, both in health and disease, it is crucial to identify the protein-protein interactions. Immunoprecipitations followed by mass spectrometry (IP-MS) have been shown to be a powerful tool to determine those interactions. However, it is often challenging to discriminate true protein interactors from contaminating proteins that bind nonspecifically to the IP beads. Many strategies have been developed to combat this issue, but very few have tackled it at the core, namely via reducing the non-specific binding of proteins onto the IP beads. Here, we describe the preparation of antifouling azidefunctionalized polymer-coated beads that can be equipped with an antibody of choice via click chemistry. We show the preparation of generic anti-GFP immunoprecipitation beads to target GFP-fusion proteins. Our antifouling anti-GFP coupled beads show similar GFP capture efficiency, but with strongly reduced non-specific protein binding, as compared to commercially available anti-GFP beads. As a result, in an IP-MS experiment many contaminating proteins were identified for the commercially available beads, whereas our antifouling beads were able to co-purify known interaction partners of the used GFP-tagged bait protein with minimal contamination. The antifouling antibody-coated beads therefore provide an excellent novel tool to identify unknown protein-protein interactions.
\end{abstract}




\subsection{Introduction}

Proteins are the workhorses of life as they take part in essentially all biological processes. In this, they rarely operate alone, but typically act in multi-protein complexes. ${ }^{1}$ To fully understand biological mechanisms, both in health and disease, it is therefore crucial to reliably identify protein interaction partners. ${ }^{2-3}$ In the last decades, along with other techniques like yeast two-hybrid, ${ }^{4-5}$ immunoprecipitation followed by mass spectrometry (IP-MS) has been proven to be a powerful tool to identify these protein-protein interactions, mainly due to the continuing improvement in accuracy and speed of mass spectrometers. ${ }^{6-7}$ In a typical IPMS experiment, a solid support with antibodies against a bait protein is used to pull-down protein complexes. The captured proteins are subsequently converted into peptides that are analyzed by mass spectrometry. ${ }^{8}$ The stable core subunits from a multi-protein complex are usually readily identified, but it remains challenging to distinguish subunits that, for example, bind non-stoichiometrically or with low affinity, from that of contaminating proteins that are non-specifically retrieved from the biological sample. ${ }^{7,9}$ In fact, the majority of proteins typically being identified during an IP-MS experiment are non-specific binders. ${ }^{7}$ The nonspecific binders most frequently originate from proteins sticking to the solid support itself, e.g. the sepharose or agarose beads, and to a smaller degree to the non-specific binding of proteins to the antibodies that are attached to the bead or to protein tags (such as GFP). ${ }^{7}, 10$

Several approaches have been developed in order to discriminate true protein-protein interactions from background noise. Stringent washing conditions are easily implemented, but these do often lead to the loss of (target) proteins precipitated from weak or transient protein interactions. ${ }^{11}$ Another common strategy is to use Stable Isotype Labeling with Amino acids in cell Culture (SILAC), ${ }^{12-13}$ in which cells containing bait proteins are grown in 'heavy' medium containing amino acids that are isotopically labeled, while negative controls cells (without bait proteins) are grown in standard 'light' medium. An IP-MS experiment can then be performed on the combined samples, the ratios between the heavy and light peaks should then indicate whether the identified protein is a true interactor or a non-specific binder. Recently also label-free quantitative methodologies have been developed that are less laborious and more suitable for high-throughput screenings than SILAC. ${ }^{9}$ Moreover, software tools have also been developed and lists of common contaminants are being compiled to assist in the data analysis and interpretation of IP-MS experiments. ${ }^{11,14-15}$ Nonetheless, the majority of proteins identified in an IP-MS experiment are still non-specific binders and specific interactions cannot always be unambiguously determined, especially close to the threshold level where signal-to-noise ratios are low. ${ }^{7} 9$ The above described methodologies all seem to take non-specific binding as an inevitable aspect of an IP-MS experiment, instead of tackling the issue at its core, via the reduction of that non-specific binding on the solid support.

Non-specific binding of biomolecules to solid surfaces, often referred to as fouling, is a recurring problem in various biomedical and bioanalytical applications. ${ }^{16}$ To prevent or reduce fouling, various types of antifouling surface coatings have been investigated, yet mainly on flat surfaces. ${ }^{17-19}$ Poly (ethylene glycol) (PEG)-based materials are the most frequently used and 
studied coatings; however, their long-term stability and performance in real-life biological fluids like blood are too limited for many biomedical applications. ${ }^{20-22}$ Polymeric zwitterionic coatings have emerged as excellent alternatives. ${ }^{16-17}$ Their outstanding antifouling properties have been attributed to the formation of an electrostatically induced hydration layer, which facilitates the repellence of proteins. ${ }^{23}$ It has been widely investigated which exact properties, ${ }^{24-27}$ e.g. nature of charged groups or thickness of polymer layer, determine the antifouling performance of a zwitterionic coating, as well as ways to functionalize the polymers with biomolecules to tune their applicability. ${ }^{28-32}$ Given their success, zwitterionic coatings are increasingly implemented in applications, for example on indwelling medical devices to reduce wear and fouling, ${ }^{33-34}$ to enhance the sensitivity of biosensing platforms, ${ }^{29,35-36}$ and for the production of antimicrobial surfaces. $^{37}$

The identification of protein-protein interactions via IP-MS procedures relies on the ability to discriminate true interactors from non-specific binders. We therefore anticipated that the incorporation of antifouling materials into the existing IP-MS methodologies would be highly valuable. By coating the solid support with an antifouling coating, similar as described previously, ${ }^{38}$ the adsorption of non-specific binders can be greatly reduced, which will make the identification of true protein interactors more reliable and straightforward.

Here we show the development of antifouling zwitterionic polymer-coated magnetic beads that can be functionalized with antibodies and subsequently be used within a standard IP-MS protocol (see Figure 5.1 for schematic representation of the IP-MS workflow). To this end, we integrated our previous work on antifouling beads (as described in chapter 3$)^{38}$ with that of copolymer brushes that incorporate azide functionalities within the still fully zwitterionic polymer layer (as described in chapter 2). ${ }^{28}$ The incorporated azide moieties were used as a reactive handle to allow efficient attachment of antibodies via click chemistry. As an example to show the potential of this approach, antibodies against GFP were used in an IP-MS experiment with nuclear extracts of HeLa cells in which a GFP-tagged Methyl-CpG-binding domain protein 3 (MBD3-GFP) was stably expressed. Our beads showed a strong reduction in non-specific protein binding in both flow cytometry and IP-MS based measurements, and thereby a highly improved performance when compared to commercially available anti-GFP beads.

\subsection{Materials and methods}

\subsubsection{Materials}

a-Bromoisobutyryl bromide (98\%), N,N-diisopropylethylamine, copper(I) chloride ( $\geq 99 \%)$, copper(II) chloride (97\%), dimethyl sulfoxide (DMSO) (anhydrous, $\geq 99.9 \%$ ), iodoacetamide and acrylamide were purchased from Sigma-Aldrich. Dimethylformamide (DMF) for peptide synthesis (99.8\%) was obtained from Acros Organics and dried over heat-activated molecular

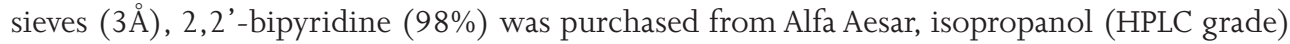
from BioSolve, and dichloromethane (DCM) from VWR International S.A.S. 
1. MIX BEADS WITH LYSATE

2. SEPARATE BEADS FROM NON-BOUND PROTEINS

\section{Standard beads}

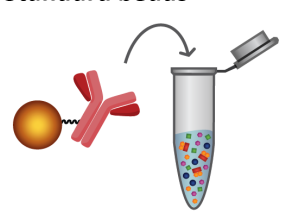

Antifouling beads

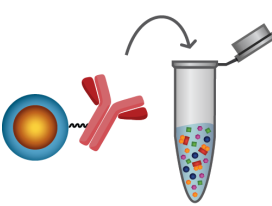

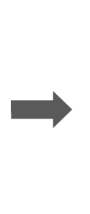

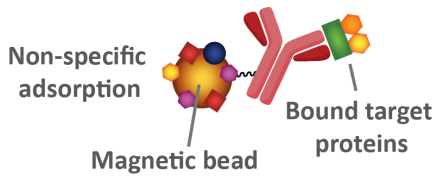

Magnetic bead

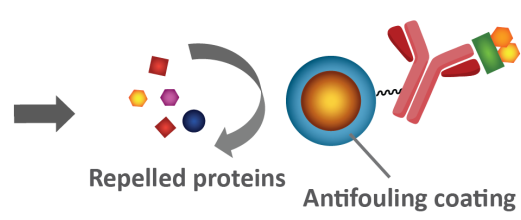

3. PROTEIN ANALYSIS: LC-MS/MS
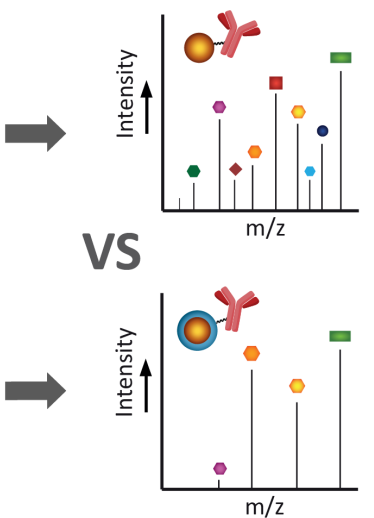

Figure 5.1 Schematic representation of IP-MS/MS workflow using antibody-functionalized magnetic beads, with or without antifouling coating: 1) The beads are mixed with a complex protein mixture. 2) Beads are separated from the protein sample using a magnet. Beads with antifouling coating bind only target proteins while other proteins are being repelled, whereas beads without antifouling coating bind target proteins, but also contain non-specifically bound proteins. 3) Subsequent protein analysis by nano LC-MS/MS shows a significant reduction in contaminating proteins for the antifouling beads.

MilliQ was produced with a MilliQ Integral 3 system (Millipore). Dynabeads (Dynabeads M-270 amine; $2.8 \mu \mathrm{m}$ diameter) were purchased from Invitrogen Life Technologies, and GFP-Trap ${ }^{\circledR} \_\mathrm{M}$ (anti-GFP $V_{\mathrm{H}} \mathrm{H}$ coupled to magnetic microparticles) and GFP-binding protein (anti-GFP $\mathrm{V}_{\mathrm{H}} \mathrm{H}$ purified protein) from Chromotek. Phycoerythrin (PE)-conjugated Goat anti-mouse IgG antibody (aMouse-PE, clone: Poly4053) was obtained from Biolegend. Bovine serum albuminAlexa Fluor 488 conjugate (BSA-AF488) and EZ-Link Sulfo-NHS-LC-Biotin were obtained from Thermo Fisher, streptavidin-phycoerythrin (Strep-PE) conjugate and streptavidin-FITC (Strep-FITC) from eBioscience. Lissamine rhodamine B $\mathrm{PEG}_{3}$ azide (Azide-lissamine) was purchased from Tenova Pharmaceuticals, and endo-BCN-PEG - NHS ester (BCN-NHS) from tebubio. Complete ${ }^{\mathrm{TM}}$ Protease Inhibitor Cocktail, trypsin and mouse monoclonal anti-GFP antibody ( $\operatorname{IgG}_{1} \kappa$, clones 7.1 and 13.1) were obtained from Roche. Bradford reagent was purchased from Bio-Rad, Fetal Calf Serum (FCS) from Gibco, Bolt ${ }^{\mathrm{TM}}$ sample buffer from Invitrogen, GelCode ${ }^{\mathbb{B}}$ Blue Stain Reagent from Thermo Scientific, ECL western blotting detection reagent from GE Healthcare and goat anti-mouse IgG antibody-HRP conjugate (AP127P) from Millipore.

\subsubsection{Synthesis}

The syntheses of 3-((3-methacrylamidopropyl)dimethylammonio)propane-1-sulfonate (SB3) monomer and 3-((3-azidopropyl)(3-methacrylamidopropyl) (methyl)ammonio)propane-1sulfonate (azido-SB) monomer were performed as described in chapter $2 .{ }^{28}$ 


\subsubsection{Bead handling}

For all collection and washing steps, beads were separated from solvent and reactants using a magnetic stand (Promega). In all cases, unless stated otherwise, reactions with beads were performed in $2 \mathrm{~mL}$ Eppendorf tubes; this allows for better bead collection when using the magnetic stand. To ensure similar amount of beads, beads were counted using a Bürker counting chamber prior to antibody immobilization, flow cytometry analysis, western blotting and IPMS.

\subsubsection{Initiator attachment}

The required amount of Dynabeads, i.e. $500 \mu \mathrm{L}$ of bead suspension as supplied by the manufacturer, was transferred to a glass tube with screwcap connection. The liquid of this bead suspension was removed using a magnet, the beads were further dried in a vacuum oven at $50{ }^{\circ} \mathrm{C}$ for $2-4 \mathrm{~h}$. The beads were resuspended in $2 \mathrm{~mL}$ of dry DCM, to this bead suspension $0.5 \mathrm{~mL}$ of $\mathrm{N}, \mathrm{N}$-diisopropylethylamine and $0.6 \mathrm{~mL}$ of $\alpha$-bromoisobutyryl bromide were added. The reaction tube was wrapped with aluminum foil and placed overnight on an end-over-end shaker at room temperature (RT). Afterwards, the beads were washed with copious amounts of DCM, washed twice with isopropanol, and subsequently twice with MilliQ water. This protocol was adapted from previous work (chapter 3 and 4$){ }^{28}$

\subsubsection{Surface-initiated polymerization}

Surface-initiated atom transfer radical polymerization (SI-ATRP) was performed as previously described (see chapter 2 and 3), but adapted to the use of beads and azido-SB monomer. ${ }^{28,38} \mathrm{All}$ steps were performed under argon atmosphere in Schlenk flasks and solutions were transferred via argon-flushed needles. A mixture of isopropanol/MilliQ water (20/80) was degassed by 5 min sonication and $30 \mathrm{~min}$ of argon bubbling. Within a glove box, $78.1 \mathrm{mg}(0.50 \mathrm{mmol})$ 2,2'-bipyridine and $23.0 \mathrm{mg}(0.23 \mathrm{mmol})$ of an $\mathrm{Cu}(\mathrm{I}) \mathrm{Cl} / \mathrm{Cu}(\mathrm{II}) \mathrm{Cl}_{2}(9 / 1)$ mixture was added to a Schlenk flask, the flask was then transferred to the fume hood where $8.2 \mathrm{~mL}$ of the degassed isopropanol/water mixture was added. The resulting mixture was stirred for $15 \mathrm{~min}$ at RT. Meanwhile, the required amount of thawed aliquot containing azido-SB monomer in MilliQ (28.9 mg, $0.08 \mathrm{mmol}$, for pSB-co-(azido) ${ }_{8 \%}$ beads) was transferred to a Schlenk flask, MilliQ from this aliquot was removed under reduced pressure before the SB3 monomer (269 $\mathrm{mg}, 0.92 \mathrm{mmol}$, for pSB-co-(azido) ${ }_{8 \%}$ beads) was added. To the monomer mixture, $900 \mu \mathrm{L}$ of brown copper/bipyridine-containing solution was added and stirred for $15 \mathrm{~min}$ at RT to fully solubilize the monomers. Initiator-functionalized Dynabeads were resuspended in $200 \mu \mathrm{L}$ of isopropanol/MilliQ mixture and bubbled with argon for $10 \mathrm{~min}$. The monomer-containing ATRP solution was transferred to the beads, the flask was closed, covered with aluminum foil and placed on a shaker at $80 \mathrm{rpm}$ for $15 \mathrm{~min}$ at RT. The reaction was stopped by opening the sample to air, pouring the solution into an Erlenmeyer flask and adding MilliQ while swirling, 
until the solution turned blue (which indicates the inactivation of the copper catalyst that typically takes $\sim 5 \mathrm{sec}$ ). The pSB-Co-(azido) ${ }_{8 \%}$-coated beads were collected using a magnet and then washed once with isopropanol/MilliQ (1/4), twice with MilliQ water and twice with PBS $\mathrm{pH}$ 7.4. The beads were stored in PBS at $4{ }^{\circ} \mathrm{C}$ until further use.

\subsubsection{Bead characterization}

\section{XPS}

X-ray photoelectron spectroscopy (XPS) samples were prepared as previously described. ${ }^{38}$ In short, beads (in MilliQ) were concentrated and dropcasted onto a piece of $\operatorname{Si}(111)$ (Siltronix, $\mathrm{N}$-type, phosphorus doped), which was cleaned by sonicating for $5 \mathrm{~min}$ in semiconductorgrade acetone followed by oxygen plasma treatment (Diener electronic, Femto A) for 5 min at $50 \%$ power. The samples were subsequently dried in a vacuum oven $(15 \mathrm{mbar})$ at $50{ }^{\circ} \mathrm{C}$ for at least $2 \mathrm{~h}$. XPS spectra were obtained using a JPS-9200 photoelectron spectrometer (JEOL, Japan) with monochromatic Al Ka X-ray radiation at $12 \mathrm{kV}$ and $20 \mathrm{~mA}$. The obtained spectra were analyzed using CASA XPS software (version 2.3.16 PR 1.6).

\subsubsection{Antibody production and purification}

Monoclonal TA99 antibody $\left(\operatorname{IgG}_{2 a}\right)$ was produced by a hybridoma cell line obtained from the American Tissue Culture Collection (ATCC $\AA$ HB-8704 ${ }^{\mathrm{TM}}$ ) that was cultured in Roswell Park Memorial Institute (RPMI) medium (Lonza), supplemented with L-glutamine, 10\% fetal calf serum (FCS), $100 \mu \mathrm{g} / \mathrm{mL}$ penicillin and $100 \mu \mathrm{g} / \mathrm{mL}$ streptomycin, at $37{ }^{\circ} \mathrm{C}$ and $5 \% \mathrm{CO}_{2}$. The antibody was isolated from the hybridoma cell line supernatant by repetitive passing of the supernatant over a Pierce Thiophilic Adsorption column (Thermo Fisher Scientific) according to the manufacturer's protocol. The concentration of the antibody was determined by a Nanodrop 1000 Spectrophotometer and checked for its purity using SDS-PAGE.

\subsubsection{Antibody functionalization}

\section{BCN-NHS}

The antibody of choice (the mouse $\operatorname{IgG}_{2 \mathrm{a}}$ TA99 or alpaca $\mathrm{V}_{\mathrm{H}} \mathrm{H}$ anti-GFP antibody) was concentrated and transferred to PBS ( $\mathrm{pH}$ 6.5) buffer using Amicon Ultra $0.5 \mathrm{~mL} 0.3 \mathrm{kDa}$ Molecular Weight Cut Off (MWCO) centrifugal filter tubes (Merck Millipore) using the manufacturer's instructions. The antibodies were subsequently labeled at a $4 \mathrm{mg} / \mathrm{mL}$ antibody concentration with an endo-BCN-PEG - -NHS ester (BCN-NHS) linker in a $1: 8$ ratio (assuming that the molecular mass of the mouse IgG TA99 is $150 \mathrm{kDa}$ and that of antiGFP is $14.1 \mathrm{kDa}$ ) by adding the BCN-NHS linker from a stock solution in which the linker was dissolved in dry DMF, to a final DMF concentration of $10 \%$. The antibody was incubated with the linker under 
ambient conditions at RT for $1 \mathrm{~h}$. The reaction was stopped by removing unreacted BCN-NHS by washing 3 times with $500 \mu \mathrm{L}$ PBS pH 6.5 using the filter tubes (in which the first 2 times 10 min centrifugation at $2100 \mathrm{~g}$ was used and the third time $30 \mathrm{~min}$ centrifugation at $2100 \mathrm{~g}$ ).

\section{AZIDE-LISSAMINE STAINING}

BCN-labeled antibodies ( $4 \mathrm{mg} / \mathrm{mL}$ ) were incubated with Azide-Lissamine in PBS pH 7.4 with an antibody/Azide-Lissamine molar ratio of $1: 20$ and a final DMSO concentration of $10 \%$. The reaction was carried out overnight under ambient conditions at RT, with the reaction mixture protected from light. The resulting reaction mixture was directly used for SDS-PAGE without further purification.

\subsubsection{Antibody attachment to beads}

BCN-labeled antibodies were attached to pSB-co-(azido) ${ }_{8 \%}$ beads $\left(\sim 50 \times 10^{6}\right.$ beads $)$ at an antibody concentration of $0.25,1$ or $4 \mathrm{mg} / \mathrm{mL}$ in $\mathrm{PBS} \mathrm{pH} 7.4$, in a final volume of $50 \mu \mathrm{L}$ in a PCR tube. The tube was fixed on an end-over-end shaker which was placed vertically, and incubated overnight at RT. To obtain homogeneous attachment it is crucial to have a proper dispersion of the beads, for further details on this, see the Supporting Information. The beads were transferred to a $2 \mathrm{~mL}$ Eppendorf tube and 3 times washed with PBS pH 7.4. The beads were stored in PBS at $4{ }^{\circ} \mathrm{C}$.

\subsubsection{Serum biotinylation}

Bovine serum was obtained and biotinylated as previously described (chapter 3 and 4 ). ${ }^{38}$ In short, sera of three adult cows were pooled and heated at $56{ }^{\circ} \mathrm{C}$ for $30 \mathrm{~min}$ (to inactivate complement proteins). Serum proteins were biotinylated using an EZ-Link Sulfo-NHS-LCBiotin reagent, using the manufacturer's instructions. Assuming that the average molecular weight of serum proteins is $70 \mathrm{kDa}, 50$ equivalents of sulfo-NHS-biotin to serum proteins was used. The reaction was carried out at RT for $60 \mathrm{~min}$. Non-bound reagents were removed using a desalting PD-10 column (Sephadex, from GE Healthcare), following the manufacturer's gravitation protocol with PBS as eluent. The concentration of the obtained biotinylated serum (Serum-Biotin) was adjusted to $10 \%$ serum solution $(\sim 6 \mathrm{mg} / \mathrm{mL})$ with PBS. Bovine blood sample collection was approved by the Board on Animal Ethics and Experiments from Wageningen University (DEC number: 2014005.b).

\subsubsection{Flow cytometry}

TA99-functionalized pSB-Co-(azido) ${ }_{8 \%}$ beads $\left(\sim 2 \times 10^{6}\right.$ beads) were incubated in PBS, BSAAF488 $(0.5 \mathrm{mg} / \mathrm{mL})$ or anti-Mouse-PE (1 : 50 dilution). Serum binding was evaluated by incubating the beads with Serum-Biotin (10\% solution) followed by Strep-FITC (1 : 200 
dilution). Specific binding of anti-Mouse-PE was evaluated by first incubating the beads in Serum-Biotin, followed by a mixture of anti-Mouse-PE and BSA-AF488 or a mixture of antiMouse-PE and Strep-FITC. All protein solutions were diluted in PBS. Anti-GFP-functionalized pSB-co-(azido $)_{8 \%}$ beads and Chromotek bead $\left(2 \times 10^{6}\right.$ beads per sample) were incubated in PBS, free GFP $(10 \mu \mathrm{g} / \mathrm{mL})$, first by Serum-Biotin followed by Strep-PE (1: 50 dilution) or a mixture of GFP and Serum-Biotin followed by Strep-PE. All incubation steps were performed for $30 \mathrm{~min}$ in $100 \mu \mathrm{L}$ of total volume on an end-over-end shaker at RT and protected from light using aluminum foil, followed by 3 times washing with $1 \mathrm{~mL}$ of PBS. The beads were subsequently resuspended in $500 \mu \mathrm{L}$ PBS, transferred to a FACS tube and analyzed with a BD FACS Canto A (BD Biosciences) flow cytometer. For each sample, 10.000 beads were measured. GFP, BSA-AF488 and Strep-FITC were visualized using the FITC channel, fouling was visualized by Strep-PE using the PE channel. Data analysis was performed using FlowJo LLC Software V10.

\subsubsection{Cells}

\section{CELL CULTURE}

Wild-type (WT) HeLa cells (HeLa B-50 from ATCC (CRL-12401)) and HeLa cells stably expressing MBD3-GFP (kindly provided by prof. dr. M.Vermeulen from the Radboud Institute of Molecular Life Sciences (RIMLS) $)^{9}$ were grown in Dulbecco's Modified Eagle Medium (DMEM, Gibco) supplemented with $10 \% \mathrm{FCS}, 100 \mu \mathrm{g} / \mathrm{mL}$ penicillin and $100 \mu \mathrm{g} / \mathrm{mL}$ streptomycin at $37{ }^{\circ} \mathrm{C}$ and $5 \% \mathrm{CO}_{2}$.

\section{CELLULAR EXTRACTS}

For whole cell lysates, cells were harvested at $\sim 90 \%$ confluency using trypsin, washed twice with cold PBS and centrifuged at $4{ }^{\circ} \mathrm{C}$ for $5 \mathrm{~min}$ at $400 \mathrm{~g}$. For whole cell extracts of WT HeLa cells, the cells were resuspended in 5 pellet volumes of cold lysis buffer $(150 \mathrm{mM} \mathrm{NaCl}, 50 \mathrm{mM}$ Tris pH 8.0, 1\% NP40 and 20\% glycerol) and incubated for $1 \mathrm{~h}$ on an end-over-end shaker at $4{ }^{\circ} \mathrm{C}$. The cell lysates were centrifuged in Eppendorf tubes for $20 \mathrm{~min}$ at $21000 \mathrm{~g}$ (maximum speed) and $4{ }^{\circ} \mathrm{C}$. The supernatants were stored at $-80{ }^{\circ} \mathrm{C}$.

\section{NUCLEAR EXTRACTS}

Nuclear extracts of WT and MBD3-GFP HeLa cells were prepared according to Smits et al. ${ }^{9}$ The cells were resuspended in 5 volumes of cold Buffer A (10 mM HEPES/KOH pH 7.9, 1.5 $\mathrm{mM} \mathrm{MgCl}, 10 \mathrm{mM} \mathrm{KCl}$ ) and incubated on ice for $10 \mathrm{~min}$ in a $15 \mathrm{~mL}$ tube. The cells were centrifuged for $5 \mathrm{~min}$ at $400 \mathrm{~g}$ and resuspended in 2 cell volumes of Buffer A, supplemented with $0.15 \%$ NP40 and complete protease inhibitor. The cells were transferred to a Dounce homogenizer and after 30-40 strokes with a Type B pestle, the resulting lysate was centrifuged for $15 \mathrm{~min}$ at $3200 \mathrm{~g}$ at $4{ }^{\circ} \mathrm{C}$. The pellet, containing the nuclei, was washed once with $1 \mathrm{~mL}$ of PBS and centrifuged for $5 \mathrm{~min}$ at $3900 \mathrm{~g}$ at $4{ }^{\circ} \mathrm{C}$. The pellet was resuspended in 2 pellet volumes of Buffer C (420 mM NaCl, $20 \mathrm{mM} \mathrm{HEPES/KOH} \mathrm{pH} \mathrm{7.9,} \mathrm{20 \%} \mathrm{v/v} \mathrm{glycerol,} 2 \mathrm{mM} \mathrm{MgCl}, 0.2$ 
mM EDTA, 0.1\% NP40, $0.5 \mathrm{mM}$ DTT and complete protease inhibitors) and transferred to an Eppendorf tube. The suspension was incubated for $1 \mathrm{~h}$ on an end-over-end shaker at $4{ }^{\circ} \mathrm{C}$ and centrifuged for $30 \mathrm{~min}$ at $18000 \mathrm{~g}$ at $4{ }^{\circ} \mathrm{C}$. The supernatants were aliquoted and stored at -80 ${ }^{\circ} \mathrm{C}$ until further use. Protein concentrations were determined using Bradford reagent.

\subsubsection{Immunoprecipitations}

For each immunoprecipitation sample, $\sim 18.6$ million beads were used, which is equal to $10 \mu \mathrm{L}$ of Chromotek bead slurry. All measurements were carried out in triplicates. WT Hela whole cell lysate was used for the evaluation of non-specific protein binding on the beads. Non-modified Dynabeads, pSB-co-(aGFP) ${ }_{8 \%}$ Dynabeads and Chromotek beads were equilibrated 3 times by magnetic separation in whole cell lysate buffer $(150 \mathrm{mM} \mathrm{NaCl}, 50 \mathrm{mM}$ Tris pH 8.0, 20\% glycerol and $1 \% \mathrm{NP} 40$ ). Next, the beads were incubated for $90 \mathrm{~min}$ at $4{ }^{\circ} \mathrm{C}$ with $1 \mathrm{mg}$ whole cell lysate. Beads were subsequently washed three times in whole cell lysis buffer, three times in PBS $+1 \%$ NP40 and three times in $50 \mathrm{mM}$ ammonium bicarbonate. After the washing steps, beads were subjected to on-bead trypsin digestion (see below).

For the co-IP-MS experiments, nuclear extracts of MBD3-GFP expressing HeLa cells and WT Hela cells were subjected to GFP enrichment using Chromotek or pSB-co- $(\mathrm{aGFP})_{8 \%}$ beads. In brief, beads were equilibrated three times in buffer $\mathrm{C}$ by magnetic separation at $4{ }^{\circ} \mathrm{C}$. After equilibration, $1 \mathrm{mg}$ of nuclear extract was added and the beads were incubated for $90 \mathrm{~min}$ at $4{ }^{\circ} \mathrm{C}$ on an end-over-end shaker. The beads were washed twice in buffer $\mathrm{C}$, twice in buffer $\mathrm{C}$ without NP40 and twice in $50 \mathrm{mM}$ ammonium bicarbonate. For mass spectrometry analysis, the beads were subjected to on-bead trypsin digestion (see below). For western blot analysis, the beads were suspended in $1 \times$ Bolt $^{\mathrm{TM}}$ sample buffer and boiled for $5 \mathrm{~min}$. The total volume of supernatant was used for western blotting and compared with $50 \mu \mathrm{g}$ of the total nuclear extract ( $5 \%$ of the total input used for IP).

\subsubsection{SDS-PAGE and Western blot}

Antibody labeling was evaluated using Any kD Mini-PROTEAN ${ }^{2}$ TGX ${ }^{\mathrm{TM}}$ Precast Protein gels and Precision Plus Protein ${ }^{\mathrm{TM}}$ Dual Color Standards from Bio-Rad, with $10 \mu \mathrm{g}$ protein per sample. Fluorescently labeled proteins were visualized using a Bio-Rad ChemiDoc ${ }^{\mathrm{TM}}$ XRS+ apparatus using the standard EtBr filter (580 AF 120 Band Pass Filter) followed by staining with GelCode ${ }^{\circledR}$ Blue Stain Reagent.

For western blot analysis of proteins subjected to IP, proteins were separated on a Bolt ${ }^{\mathrm{TM}}$ bis-tris 4-12\% SDS-PAGE gradient gel (Invitrogen). After SDS-PAGE separation proteins were blotted on a nitrocellulose membrane using the transblot turbo transfer system (Bio-Rad). After the transfer, the membrane was blocked in 5\% skimmed milk powder in tris-buffered saline Tween-20 (TBST) for $45 \mathrm{~min}$ at $25{ }^{\circ} \mathrm{C}$. The membrane was then incubated overnight at $4{ }^{\circ} \mathrm{C}$ with a mouse monoclonal anti-GFP antibody. After overnight incubation, the membrane 
was washed trice in TBST and incubated with a goat anti-mouse IgG antibody-HRP conjugate for 60 min at RT. The membrane was washed trice in TBST and imaged on a ChemiDoc ${ }^{\mathrm{TM}} \mathrm{XRS}$ (BioRad) using ECL western blotting detection reagent.

A
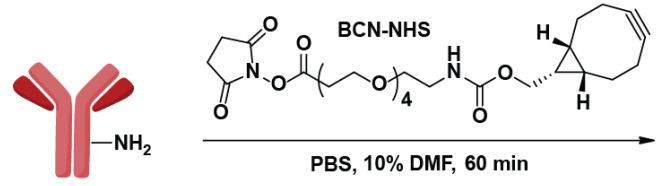

PBS, 10\% DMF, $60 \mathrm{~min}$

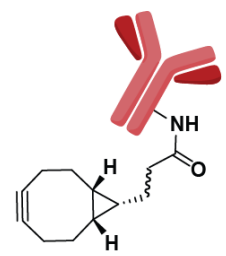

B
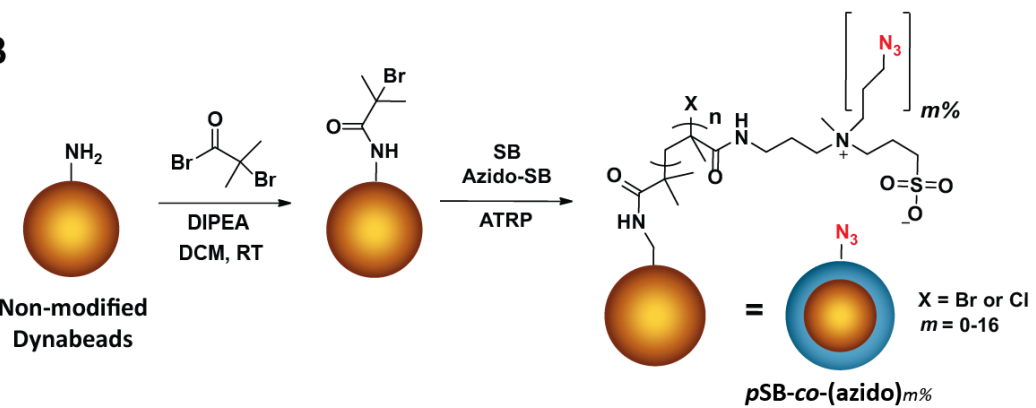

C

Non-modified

Dynabeads

DCM, RT
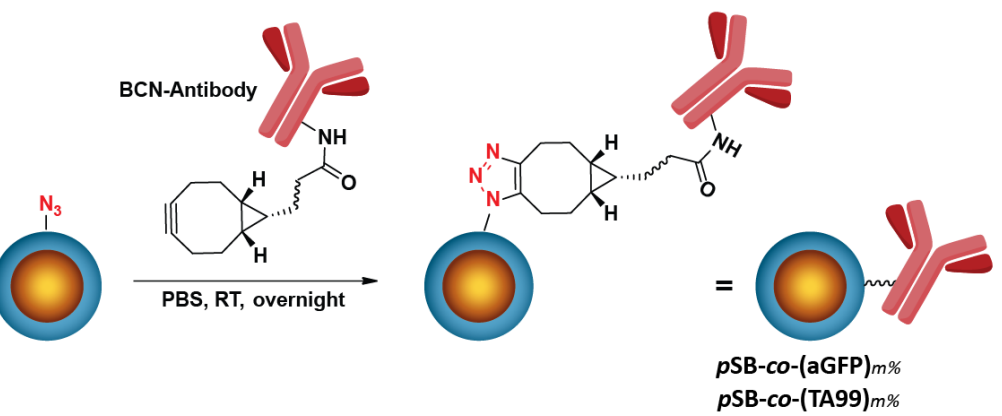

Scheme 5.1 Overview of chemical modifications of antibodies and amine-terminated beads to yield magnetic antifouling beads with coupled antibodies. Notes: (A) Functionalization of antibodies using endo-BCN-PEG - -NHS ester (BCN-NHS) linker.The NHS part of the linker reacts with free primary amines of the antibody to yield BCN-functionalized antibodies. (B) Installation of the ATRP initiator by reacting $\alpha$-bromoisobutyryl bromide with amine-terminated Dynabeads, followed by the copolymerization of a standard sulfobetaine (SB) with an azide-functionalized sulfobetaine (Azido-SB) using ATRP, to generate antifouling beads with $\mathrm{m} \%$ of azide moieties. (C) Combining the BCN-functionalized antibodies from (A) with the functional antifouling beads of (B) via the Strain-Promoted Azide-Alkyne Cycloaddition (SPAAC) reaction of BCN with the incorporated azides, to create antibody-functionalized beads. 


\subsubsection{Sample preparation for mass spectrometry}

Bead-precipitated proteins were subjected to on-bead trypsin digestion as described by Smith et al. ${ }^{9}$ In brief, beads were resuspended in elution buffer (2 M urea, $100 \mathrm{mM}$ Tris pH 7.5 and 10 $\mathrm{mM}$ DTT) and incubated for $20 \mathrm{~min}$ at $25^{\circ} \mathrm{C}$. After $20 \mathrm{~min} 50 \mathrm{mM}$ acrylamide was added and further incubated for $30 \mathrm{~min}$ at $25{ }^{\circ} \mathrm{C}$ in the dark. After alkylation, $0.35 \mu \mathrm{g}$ of trypsin was added and incubated overnight at $25{ }^{\circ} \mathrm{C}$. After overnight trypsin digestion, the obtained peptides were desalted and concentrated by $\mathrm{C} 18$ Stagetips according to Rappsilber et al., ${ }^{39}$ with the modification that on top of the Stagetips, $1 \mathrm{mg}$ LiChroprep C18 beads were used. After Stagetip processing, peptides were applied to online nano LC-MS/MS using a $60 \mathrm{~min}$ acetonitrile gradient from 8-50\%. Spectra were recorded on a LTQ-XL mass spectrometer (Thermo Scientific) and analyzed according to Wendrich et $\mathrm{al}^{40}$ Data visualization was performed in Adobe Illustrator and R. The data are represented as volcano plots in which the $-{ }^{10} \log$-transformed P-values of a False Discovery Rate (FDR)-corrected t-test are plotted against the ${ }^{2} \log$ of the relative label-free quantification (LFQ) intensities. ${ }^{40}$ Identified proteins are considered significant (black dots) with a ${ }^{2} \log$ fold-change $(\mathrm{FC})>2$ and a $\mathrm{P}$-value $<0.05$, as indicated by the gray lines.

\subsection{Results and Discussion}

\subsubsection{Zwitterionic polymer-coated beads with azide functionality}

To obtain antifouling polymer-coated microbeads we used Surface-Initiated Atom Transfer Radical Polymerization (SI-ATRP), ${ }^{41}$ in which the zwitterionic polymer brushes are grown from the surface via controlled radical polymerization. SI-ATRP has become the method of choice to create high-performance antifouling layers, as it yields densely packed coatings of tunable thicknesses. ${ }^{16,42}$ We combined here our previous work on antifouling beads made by SI-ATRP $^{38}$ with that on azide-functionalized coatings on flat surfaces ${ }^{28}$ (see Scheme 5.1B). The azide functionalities can be incorporated by the copolymerization of a standard zwitterionic sulfobetaine monomer (SB) with that of a tailor-made azide-functionalized sulfobetaine (azido$\mathrm{SB})$, enabling the incorporation of azide moieties at the desired percentages while retaining a fully zwitterionic brush.

As the first step an ATRP initiator was installed by reacting amine-terminated magnetic Dynabeads with bromoisobutyryl bromide as similarly as described in chapter 3 . This was followed by the copolymerization of the standard SB monomer with azido-SB, with azidoSB percentages $m=0$ to $16 \%$, to obtain $\mathrm{pSB}$-CO-(azido) ${ }_{\mathrm{m} \%}$ beads. The successful growth of the copolymer brushes was confirmed by XPS, showing the appearance of two sulfur peaks (168 eV for S 2p, $232 \mathrm{eV}$ for S 2s) in the XPS wide scan, that originate from the negatively charged sulfonate group (Figure 5.2A, pSB-co-(azido) ${ }_{8 \%}$ beads). The XPS C 1s spectrum shows the C-C peak at $285.0 \mathrm{eV}$, the carbon atoms next to a heteroatom (C-O, C-N, and $\mathrm{C}-\mathrm{S})$ at $286.2 \mathrm{eV}$ 
and the carbonyl peak at $287.6 \mathrm{eV}$. The experimentally derived percentages of $48.4 \%(\mathrm{C}-\mathrm{C})$, $41.5 \%(\mathrm{C}-\mathrm{O}, \mathrm{C}-\mathrm{N}$, and $\mathrm{C}-\mathrm{S})$ and $10.1 \%$ (carbonyl) fit well to the theoretical percentages of $50 \%, 41.6 \%$ and $8.3 \%$, respectively. The $1: 1$ ratio of the positively charged ammonium $(402.4$ $\mathrm{eV})$ and amide $(399.5 \mathrm{eV})$ peaks that is characteristic for these amide-linked zwitterionic moieties is clearly seen in the XPS N 1s spectrum (Figure 5.2C), confirming polymer brushes of sufficient thickness. ${ }^{38}$ Moreover, from deconvolution of the $\mathrm{N} 1 \mathrm{~s}$ spectrum of pSB-co(azido) ${ }_{8 \%}$ beads, two additional peaks (400.8 and $404.6 \mathrm{eV}, 2: 1$ ratio) can be discerned that correspond to the azide functionalities of the incorporated azido-SB monomers. Based on the area of those azide peaks, it follows that $7 \%$ of azido-SB was incorporated within the polymer brushes, which is close to the aimed 8\% azido-SB. The azide peaks fitted in Figure 5.2C are near the limit of detection by XPS; for more pronounced azide peaks see Supporting Information
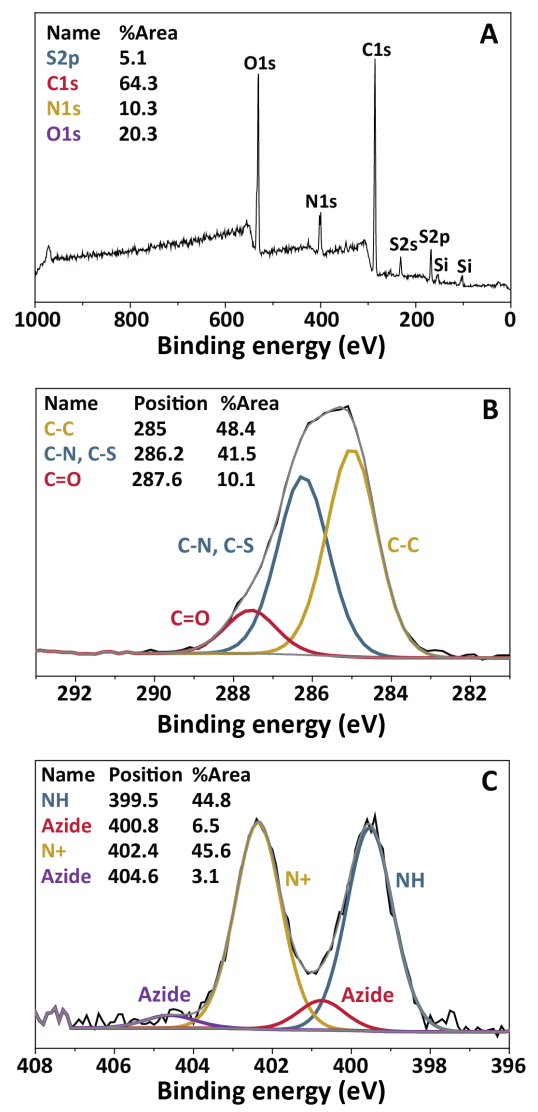

Figure 5.2 (A) XPS wide scans, (B) XPS C 1s narrow scans and (C) XPS N 1s narrow scans of pSB-cO(azido) ${ }_{8 \%}$ beads. The spectra show the successful growth of zwitterionic copolymer brushes of $\mathrm{SB}$ and azido-SB monomer from Dynabeads. The Si peaks in the wide scans correspond to the silicon substrate onto which the beads were drop casted prior to XPS analysis. 
Figure S5.3, which shows the N 1s spectrum of pSB-co-(azido) ${ }_{16 \%}$ beads. In line with this, the azide signals of $\mathrm{pSB}-\mathrm{co}$-(azido) ${ }_{\mathrm{m} \%}$ beads with $\mathrm{m} \leq 4 \%$ were too low to be detected in the XPS 1 Ns spectra (data not shown). The XPS wide scans and the C and N 1s spectra of pSB-Co-(azido) $\mathrm{m} \%$ beads are virtually identical to previously shown azido-SB brushes on flat surfaces (Figure 2.3 and Figure S2.7). ${ }^{28}$

\subsubsection{Antibody coupling to antifouling beads}

The introduction of azide moieties within the antifouling coating allows for the functionalization of the polymer brushes via click chemistry, e.g. by the Copper-Catalyzed Azide-Alkyne Cycloaddition (CuAAC) ${ }^{43}$ or Strain-Promoted Azide-Alkyne Cycloaddition (SPAAC) ${ }^{44}$ We have previously shown the incorporation of mannose and biotin on zwitterionic sulfobetaine polymer-coated beads via CuAAC and SPAAC, respectively (see chapter 3). ${ }^{38}$ To use the antifouling beads for immunoprecipitations, the beads need to be functionalized with antibodies. A commercially available BCN-NHS linker was chosen to couple antibodies to the pSB-co-(azido) $)_{\mathrm{m} \%}$ beads (see Scheme 5.1). The primary amines of the antibody can react with the NHS group to form a stable covalent amide bond, while the BCN moiety can react with the azides within the polymer layer via the aforementioned SPAAC reaction, to form a stable triazole ring. Antibodies were first reacted with the BCN-NHS linker followed by reacting the BCN-antibody conjugate with pSB-Co-(azido) ${ }_{m \%}$ beads, as this allows for the removal of unreacted or hydrolyzed BCN-NHS linker from the samples. When the linker would be first attached to the beads (overnight reaction) the NHS group could get hydrolyzed, lowering the amount of attachment sites for the antibody onto the beads. Moreover, unreacted NHS sites after antibody coupling would need to be blocked to prevent unspecific protein coupling to the beads in later steps. Both inconveniencies are avoided when first the antibodies are reacted with the linker; the remaining linker is then washed away before coupling the antibodies to the beads. In addition, to prevent antibody aggregation and obtain sufficient labeling, it turned out that the antibody concentration and $\mathrm{pH}$ during the reaction were both crucial. From pilot experiments it was established that the best results were obtained when the BCN-NHS labeling of antibodies was carried out at $\mathrm{pH} 6.5$ at $4 \mathrm{mg} / \mathrm{mL}$ antibody concentration.

The monoclonal mouse $\operatorname{IgG}_{2 \mathrm{a}}$ TA99 antibody against tyrosinase-related protein 1 (TYRP1), ${ }^{45}$ which is expressed on melanoma cells, was used as a model antibody to optimize the antibody coupling to pSB-CO-(azido) ${ }_{\mathrm{m} \%}$ beads. The attachment of the BCN-NHS linker to obtain BCN-TA99 was evaluated by reacting the BCN-TA99 conjugate with an azide-functionalized fluorophore: Azide-Lissamine. Unmodified TA99, BCN-TA99 and Lissamine-TA99 were loaded on an SDSPAGE gel, after which a fluorescence image was taken to visualize the Lissamine, followed by Coomassie blue staining (see Figure 5.3; see Supporting Information Figure S5.4 for full-size gel images). The Coomassie-stained gel showed clear bands corresponding to the heavy (50 $\mathrm{kDa})$ and the light chain $(25 \mathrm{kDa})$ of the TA99 antibody in all samples. The fluorescence image only showed the heavy and light chain of Lissamine-TA99, confirming the successful reaction of BCN-NHS with TA99 and the subsequent reaction of BCN-NHS with Azide-Lissamine. 
A

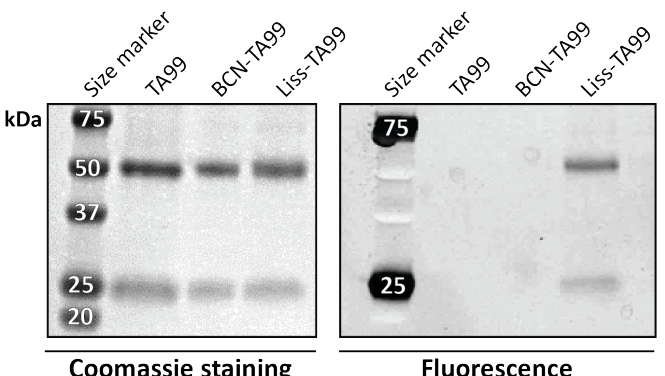

B Antibody attachment

Fouling

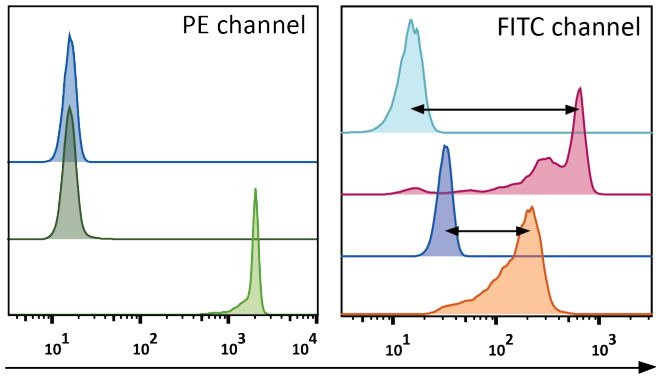

Fluorescence intensity

$\square$ pSB + PBS $\square$ pSB + aMouse $\square$ pSB-TA99 + aMouse

$\square \mathrm{NM}+$ PBS $\square \mathrm{NM}+$ Serum $\square$ pSB-TA99 + Serum
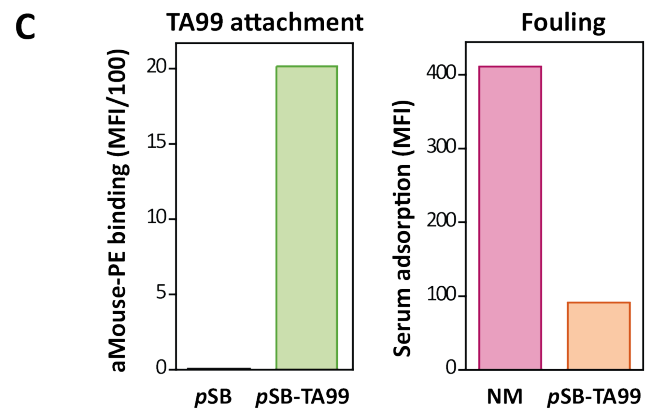

Figure 5.3 Coupling efficiency and antifouling performance of TA99 antibody to pSB-co-(azido) ${ }_{8 \%}$ (pSB) beads. (A) Evaluation of BCN-NHS coupling to TA99 antibody by SDS-PAGE analysis. Untreated TA99 antibody, TA99 labeled with BCN-NHS (BCN-TA99), and BCN-TA99 reacted with Azide-Lissamine (LissTA99) are visualized by fluorescence and Coomassie blue staining. (B) Representative flow cytometry data of non-modified Dynabeads (NM), pSB-co-(azido) ${ }_{8 \%}(\mathrm{pSB})$ and pSB-co-(TA99) ${ }_{8 \%}$ (pSB-TA99) beads measured for their antibody attachment and antifouling performance. The TA99 antibody attachment is visualized using an aMouse-PE antibody, fouling is measured by incubating the pSB-co-(TA99) ${ }_{8 \%}$ beads in $10 \%$ Serum-Biotin followed by staining with Strep-FITC. (C) Flow cytometry data of (B) depicted as bar plot based on the median fluorescent intensities (MFI). MFI values were corrected by subtracting the corresponding auto-fluorescence values of beads incubated in PBS. 
Subsequently, the attachment of BCN-TA99 to pSB-Co-(azido) ${ }_{\mathrm{m} \%}$ beads to create pSB-Co-(TA99) ${ }_{\mathrm{m} \%}$ beads was investigated using flow cytometry. The percentage of azido-SB was varied from $\mathrm{m}=0,2,4,8$ to $16 \%$, and the TA99 antibody concentration during the coupling reaction to beads was varied from $0.5,1,2,4$ to $8 \mathrm{mg} / \mathrm{mL}$ (see Supporting Information Figure S5.8 and Figure S5.9). The antibody attachment was evaluated by fluorescence using a phycoerythrinconjugated goat anti-mouse antibody (aMouse-PE). Figure 5.3B displays pSB-CO-(TA99) ${ }_{8 \%}$ beads that were made using a $4 \mathrm{mg} / \mathrm{mL}$ BCN-TA99 antibody concentration. A clear shift in fluorescence intensity was seen for PSB-CO-(TA99) ${ }_{8 \%}$ beads stained with aMouse-PE as compared to pSB-co-(azido $)_{8 \%}$ beads in PBS, indicating successful attachment of TA99 onto the polymercoated beads. Staining pSB-CO-(azido) ${ }_{8 \%}$ beads with aMouse-PE did not result in any change in fluorescence intensity, excluding the possibility of non-specific binding of aMouse-PE antibody to the polymer-coated beads.

The antifouling performance of the beads was examined by staining with fluorescently labeled BSA (BSA-AF488) or biotinylated serum (Serum-Biotin) followed by fluorescently labeled streptavidin (Strep-FITC). pSB-co-(azido) ${ }_{8 \%}$ and pSB-CO-(TA99) ${ }_{8 \%}$ beads did not show any increase in BSA-AF488 binding as seen by the fluorescence intensity within the FITC channel, when compared to the corresponding beads incubated in PBS (Figure S5.7), proving their antifouling behavior against single-protein solutions. The beads were also incubated in the more challenging 10\% biotinylated serum solution. This serum solution has a 12 times higher total protein concentration than the BSA-AF488 solution, and also contains a whole range of other proteins of which several, like naturally occurring immunoglobulins, were found on PEG-based surfaces that were exposed to blood plasma. ${ }^{46}$ PSB-Co-(TA99) ${ }_{8 \%}$ beads stained with Serum-Biotin followed by Strep-FITC showed an increase in fluorescence as compared to the PBS control (Figure 5.3). However, the increase is significantly less (factor five based on the median fluorescence intensity (MFI)) than the non-specific adsorption of serum proteins on non-modified Dynabeads (NM). Despite the attachment of an antibody, the antifouling properties of the pSB-co-(azido $)_{8 \%}$ beads could therefore largely be maintained. We choose pSB-co-(azido $)_{8 \%}$ beads combined with antibody coupling at $4 \mathrm{mg} / \mathrm{mL}$ as the optimal balance between high-loading of antibody versus fouling.

\subsubsection{Antifouling anti-GFP beads}

To make a generic bead suitable for IP-MS experiments, pSB-co-(azido) ${ }_{8 \%}$ beads were functionalized with antibodies against the Green Fluorescence Protein (GFP). GFP is widely used, among many other applications, in the form of a fusion protein as an epitope $\operatorname{tag}^{12,47} \mathrm{~A}$ genetic fusion between a gene of interest and the gene encoding GFP is readily obtained using standard molecular cloning techniques; the fused genes can subsequently be expressed in cells or whole organisms. ${ }^{47-48}$ The GFP fusion proteins may then be used for multiple purposes including the visualization of dynamic cellular events, determination of protein localization and for biochemical pull-down assays including co-immunoprecipitations. ${ }^{9}{ }^{49}$ The use of an epitope tag like GFP or His for IP-MS experiments has several advantages: excellent antibodies against e.g. GFP are readily available, whereas the availability of antibodies against endogenous proteins 

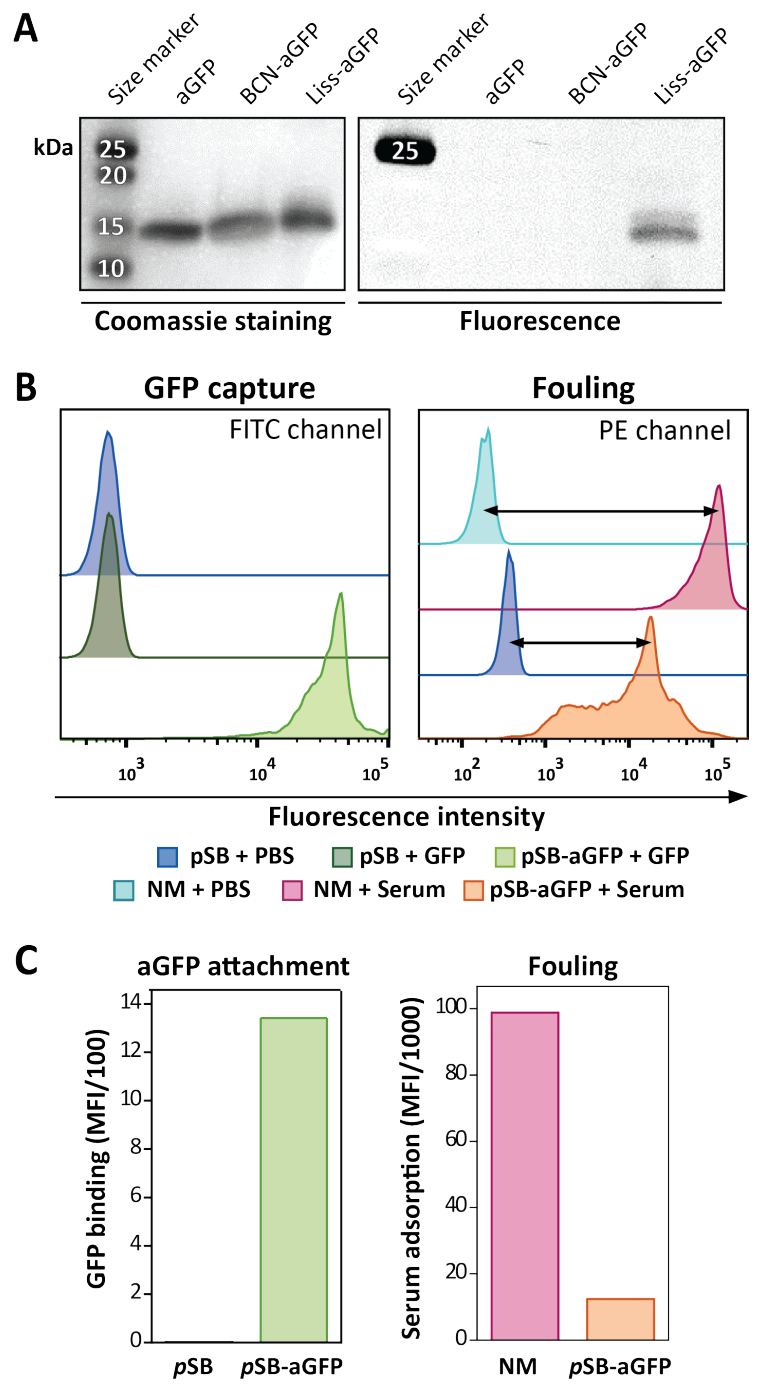

Figure 5.4 Coupling efficiency and antifouling performance of aGFP antibody to pSB beads. (A) Evaluation of BCN-NHS coupling to aGFP by SDS-PAGE analysis. Untreated aGFP antibody, aGFP labeled with BCN-NHS (BCN-aGFP), and BCN-aGFP reacted with Azide-Lissamine (Liss-aGFP) are visualized by fluorescence and Coomassie blue staining. (B) Flow cytometry data of pSB-co-(azido) ${ }_{8 \%}$ (pSB) beads, pSB-co-(aGFP) ${ }_{8 \%}(\mathrm{pSB}-\mathrm{aGFP})$ beads prepared using a $4 \mathrm{mg} / \mathrm{mL}$ aGFP concentration and non-modified Dynabeads (NM). aGFP antibody attachment is visualized by capturing free GFP and fouling by incubating with $10 \%$ Serum-Biotin followed by staining with Strep-PE. (C) Flow cytometry data of (B) depicted as bar plot based on the median fluorescent intensities (MFI). MFI values are corrected for auto-fluorescence by subtracting MFI values of beads incubated in PBS. 
is limited and they vary highly in affinity and specificity. Besides that, despite its relative large size of $27 \mathrm{kDa}$, GFP itself has no major contribution in the co-purification of contaminating proteins. ${ }^{12}$ We therefore generated a generic antifouling bead that can be used to pull-down GFP-fusion proteins by attaching an antibody against GFP (aGFP) to pSB-co-(azido) ${ }_{8 \%}$ beads. To minimize the increase of non-specific binding upon loading the zwitterionic coating with proteins, ${ }^{50}$ only the variable domains $\left(\mathrm{V}_{\mathrm{H}} \mathrm{H}\right.$ domain) from an anti-GFP heavy chain antibody - i.e. the part that actually binds to the antigen - also known as nanobodies obtained from camelids, ${ }^{51}$ were used to capture GFP.

Similar to TA99, aGFP was labeled with BCN-NHS at a $1: 8$ antibody to linker ratio and the reaction was evaluated using Azide-Lissamine, followed by SDS-PAGE analysis (see Figure 5.4). As can be seen from the fluorescence image, Liss-aGFP is visible at the expected size of $14.1 \mathrm{kDa}$, indicating successful labeling of aGFP by BCN-NHS. The BCN-aGFP antibody was then coupled to $\mathrm{pSB}$-co-(azido $)_{8 \%}$ beads to obtain $\mathrm{pSB}-\mathrm{CO}-(\mathrm{aGFP})_{8 \%}$ beads and those beads were then analyzed by flow cytometry (Figure 5.4). The attachment of BCN-aGFP to the beads was indirectly proven by capturing free GFP, which was spiked in biotinylated serum. Compared to the PBS control, there is no detectable shift in fluorescence of GFP to the antifouling pSB-co-(azido) ${ }_{8 \%}$ beads without coupled anti-GFP, demonstrating that GFP does not adsorb in a non-specific manner. With the pSB-CO-(aGFP) ${ }_{8 \%}$ beads however, there is a clear increase in fluorescence intensity and therefore a significant amount of selectively captured GFP. The antifouling performance of the pSB-CO- (aGFP) ${ }_{8 \%}$ beads was measured by staining the in Serum-Biotin incubated beads with Strep-PE. Similar to pSB-co-(TA99) ${ }_{8 \%}$ beads fouling was observed, but significantly less than on non-modified Dynabeads (factor eight). With the pSB-Co-(aGFP) ${ }_{8 \%}$ beads a factor eight less fouling was observed compared to non-modified beads, while with pSB-cO-(TA99) ${ }_{8 \%}$ beads a factor five less fouling was obtained. This can most likely be attributed to the aforementioned smaller size of the aGFP antibody as compared to TA99, which aids to reduce the amount of non-specific protein binding.

Here and in previous work (chapter 3) ${ }^{38}$ we have established that the pSB-co-(azido) ${ }_{\mathrm{m} \%}$ beads prevent or reduce fouling significantly compared to corresponding non-modified beads. To benchmark the performance of pSB-CO- $(\mathrm{aGFP})_{8 \%}$ beads, both regarding GFP binding efficiency and antifouling properties, a comparison was made with commercially available magnetic Chromotek GFP-Trap ${ }^{\circledR}$ beads. These beads are specifically intended for immunoprecipitation, and are already coated with anti-GFP antibodies, and these and similar type of beads are often used for immunoprecipitations targeting GFP-fusion proteins. ${ }^{9,52-53}$ Our pSB-CO-(aGFP) ${ }_{8 \%}$ beads were prepared with the exact same alpaca anti-GFPV ${ }_{\mathrm{H}} \mathrm{H}$ domain antibody as on the Chromotek beads, enabling a fair comparison between the two types of beads. The Chromotek beads were compared with pSB-CO-(aGFP) ${ }_{8 \%}$ beads that were prepared at a BCN-aGFP concentration of 0 , $0.25,1$ or $4 \mathrm{mg} / \mathrm{mL}$. The $\mathrm{pSB}-\mathrm{CO}-(\mathrm{aGFP})_{8 \%}$ beads prepared at $0 \mathrm{mg} / \mathrm{mL}$ are identical to the $\mathrm{pSB}-$ CO-(azido $)_{8 \%}$ beads. All beads were incubated with $10 \%$ biotinylated serum solution, which was spiked with free GFP, followed by a subsequent staining with Strep-PE. The Chromotek beads appear less monodisperse than the Dynabeads (see Supporting Information Figure S5.10), as can be seen from the forward and side scatter flow cytometry plots. The forward scattering is a measure for the size of the particles being measured. Based on size, clear distinct groups 
(populations) could be discriminated for single, double and multiple Dynabeads. Chromotek beads on the other hand show such large diversity in size that it was not possible to only select (gate for) the single beads. In order to make a fair comparison between the Dynabeads and Chromotek beads, all beads were used for further analysis, whether singlets or not.

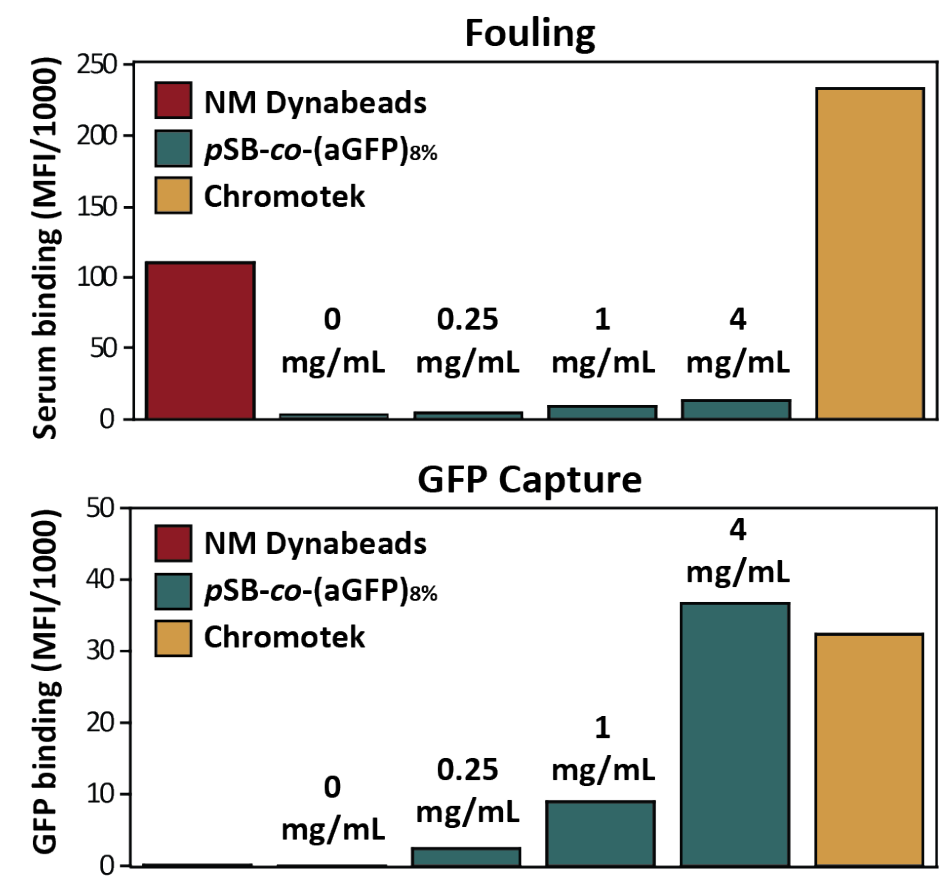

Figure 5.5 Flow cytometry data of non-modified Dynabeads, pSB-co-(aGFP) ${ }_{8 \%}$ beads prepared with an aGFP antibody concentration of $0,0.25,1$ or $4 \mathrm{mg} / \mathrm{mL}$, and commercial aGFP Chromotek beads. All beads were incubated in PBS or in a protein mixture of free GFP $(10 \mu \mathrm{g} / \mathrm{mL})$ and serum proteins ( $10 \%$ Serum-Biotin), followed by staining with Strep-PE. Serum binding was measured in the PE channel whereas GFP capture was measured using the FITC channel. Median Fluorescence Intensities (MFI) of each sample were corrected by subtracting the PBS values of their corresponding samples.

For each bead event measured by flow cytometry, fouling in the PE channel and GFP capture in the FITC channel were measured simultaneously. Figure 5.5 summarizes the intensities of 10,000 bead events per sample. The depicted median fluorescence intensities (MFI) were corrected for the MFI of beads incubated in PBS. All pSB-CO-(aGFP) ${ }_{8 \%}$ beads show substantial reduction in fouling compared to non-modified Dynabeads, and a slight increase in fouling with increasing amount of attached antibody. Chromotek beads showed to be even more fouling than non-modified Dynabeads. When comparing the amount of captured GFP, there is negligible non-specific binding of GFP to non-modified Dynabeads and pSB-co-(azido) ${ }_{8 \%}$ beads 
(the $0 \mathrm{mg} / \mathrm{mL}$ sample), but clear GFP binding by all three $\mathrm{pSB}-\mathrm{CO}-(\mathrm{aGFP})_{8 \%}$ beads in an almost linear relationship. The amount of captured GFP protein by Chromotek beads was comparable to pSB-co- $(\mathrm{aGFP})_{8 \%}$ beads prepared with $4 \mathrm{mg} / \mathrm{mL}$ aGFP. In the subsequent immunoprecipitation experiments we therefore used the $4 \mathrm{mg} / \mathrm{mL}$ pSB-co- $(\mathrm{aGFP})_{8 \%}$ beads.

In Figure 5.5 (aGFP) and Figure S5.8 (TA99) it was observed that coupling of an increased amount of antibody to the beads, leads to increased fouling on those beads. In other words, when using a highly efficient antifouling coating the amount of fouling is largely determined by the amount of attached antibody. The amount of antibody coupled to the beads can be varied in three ways: a) by changing the percentage of incorporated azido-SB (higher percentages leads to more antibody attachment, see Supporting Information Figure S5.8), b) by varying the antibody concentration during the coupling of the BCN-antibody to the azide-bearing beads (higher concentration leads to more antibody attachment, Figure 5.5 and Figure S5.9), and c) by changing the BCN-NHS to antibody ratio (data not shown). Depending on the application, the balance between on the one hand high antibody loading and corresponding binding capacity, and on the other hand fouling might differ and thus can be optimized accordingly.

\subsubsection{Immunoprecipitations using antifouling beads}

Since pSB-co-(aGFP) ${ }_{8 \%}$ beads could capture similar amounts of GFP as commercial Chromotek GFP-Trap ${ }^{\circledR}$ beads (Figure 5.5) - but with much less non-specific binding - it was anticipated that the pSB-co- $(\mathrm{aGFP})_{8 \%}$ beads had sufficient antibody loading for their use in immunoprecipitations in which GFP-fusion proteins are captured from cellular extracts at endogenous concentrations. To test this, nuclear extracts were prepared from WT and MBD3GFP expressing HeLa cell lines. The MBD3-GFP transgene was created using bacterial artificial chromosome (BAC) recombineering to ensure near endogenous MBD3-GFP protein expression to avoid artifacts associated with overexpression. ${ }^{48,54}$ The MBD3 protein is known to bind to hydroxymethylated DNA and assembles in the Nucleosome Remodeling and Deacetylase (NuRD) complex. The MBD3/NuRD complex has been well characterized ${ }^{55-56}$ and Chromotek beads have been previously used for immunoprecipitations targeting MBD3-GFP, followed by label-free quantitative mass spectrometry-based protein analysis. ${ }^{9}$ Hence, targeting MBD3-GFP and its interaction partners by commercial Chromotek beads serves as an adequate approach by which we can compare our novel pSB-CO- $(\mathrm{aGFP})_{8 \%}$ beads.

The ability of pSB-CO-(aGFP) ${ }_{8 \%}$ beads to capture MBD3-GFP in an immunoprecipitation experiment was first investigated by incubating the beads in MBD3-GFP-containing nuclear extract, the proteins that were bound by the beads were analyzed by western blotting (see Figure 5.6). After the immunoprecipitation, a thick and clear GFP band is seen (the band indicated as 'bound') which demonstrates that the pSB-CO-(aGFP) ${ }_{8 \%}$ beads are capable of capturing GFPfusion proteins at endogenous expression levels. These positive results prompted us to use the pSB-co-(aGFP) ${ }_{8 \%}$ beads in an comparative IP-MS experiment with Chromotek beads targeting MBD3-GFP. 
To evaluate the amount of non-specific binding on non-modified, pSB-co-(azido) ${ }_{8 \%}$ and Chromotek beads in an immunoprecipitation experiment, the beads were first incubated in whole cell lysate prepared from WT Hela cells (i.e. without the MBD3-GFP target protein) and analyzed by nano LC-MS/MS. The data were visualized in volcano plots (see Figure 5.7A and $5.7 \mathrm{~B}$ ) in which the statistical significance of identified proteins, represented as dots, is plotted against the relative label-free quantification intensities (LFQ) between two samples. In other words, the $x$-axis shows the relative difference between the samples where the protein intensities of a first sample is related to that of a second sample and expressed as an exponent of two. Statistical significant proteins are presented as black dots, the grey lines represent the set thresholds. In whole cell lysates of WT Hela cells, 47 non-specifically bound proteins were identified as being significant for the non-modified Dynabeads as compared to 6 proteins for the pSB-co-(azido) ${ }_{8 \%}$ beads. Likewise, 71 proteins were identified using Chromotek beads compared to 18 proteins with pSB-CO-(azido) ${ }_{8 \%}$ beads. This shows also by IP-MS that the azidoSB coating greatly reduces the amount of non-specifically bound proteins, which were - in an analysis of the individual dots in the volcano plot - largely identified as ribosomal proteins.

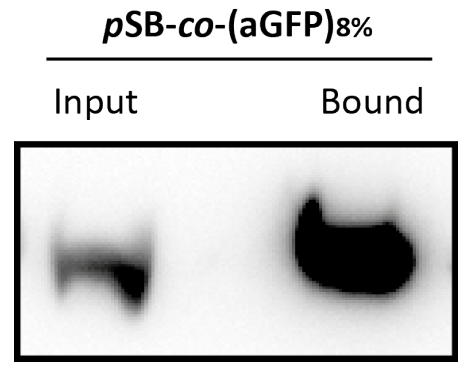

Figure 5.6 Western blot analysis before (input) and after immunoprecipitation (bound) with pSBCo- $(\mathrm{aGFP})_{8 \%}$ beads and nuclear extracts of MBD3-GFP expressing HeLa cells. The input loaded on gel corresponds to $5 \%$ of the total amount of nuclear extract that was used before immunoprecipitation. The total amount of bound proteins was loaded on gel after immunoprecipitation

Having established a reduced amount of fouling on pSB-CO-(aGFP) ${ }_{8 \%}$ beads as compared to the Chromotek beads, the beads were subjected to co-IP-MS using MBD3-GFP-containing HeLa extracts. In an ideal co-IP experiment, only the bait protein (in this case the MBD3-GFP protein) and its interaction partners are detected, as any other protein might incorrectly be acknowledged as a binding partner of the bait protein. When using tagged/fusion proteins as bait protein for IP-MS, it is common practice to minimize the risk of false positives by using as a control a protein extract identical to that of the bait protein, but without the tagged bait protein itself. ${ }^{9}$ Only the proteins that are enriched (i.e. with a ${ }^{2} \log (\mathrm{FC})>2$ ) in the protein extract expressing the tagged bait protein, are considered as valuable candidates. The same methodology was followed here: pSB-CO- $(\mathrm{aGFP})_{8 \%}$ and Chromotek beads were separately incubated in nuclear extracts of MBD3-GFP expressing HeLa cells (bait samples) as well as in 
A

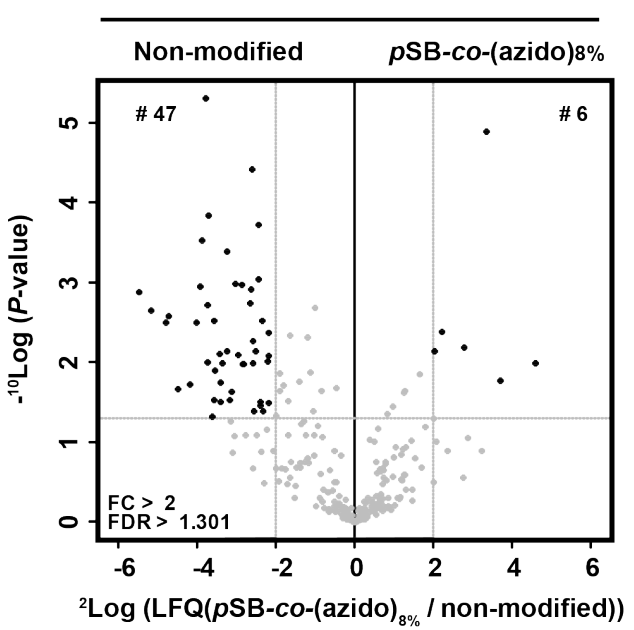

C

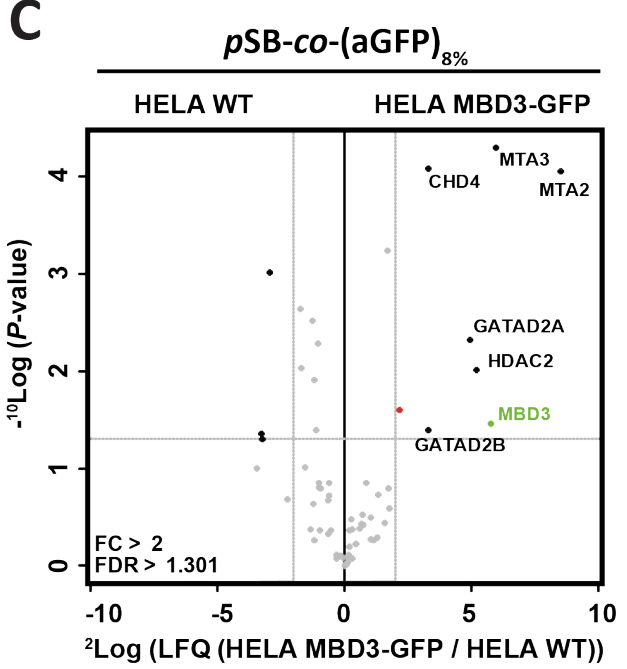

B

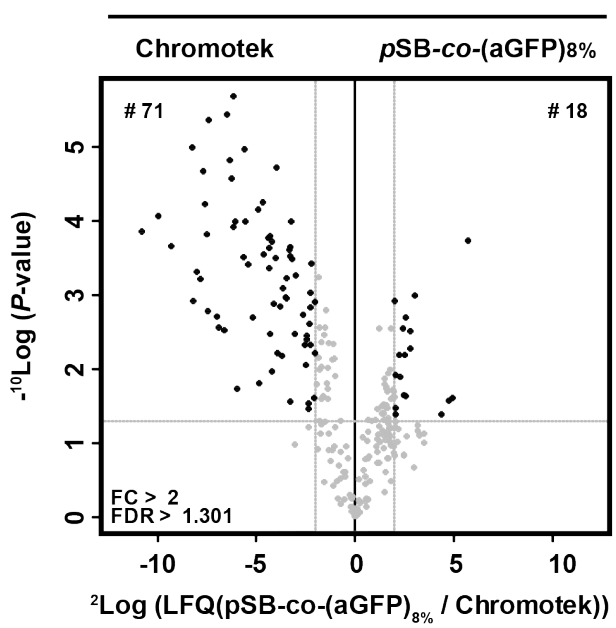

D

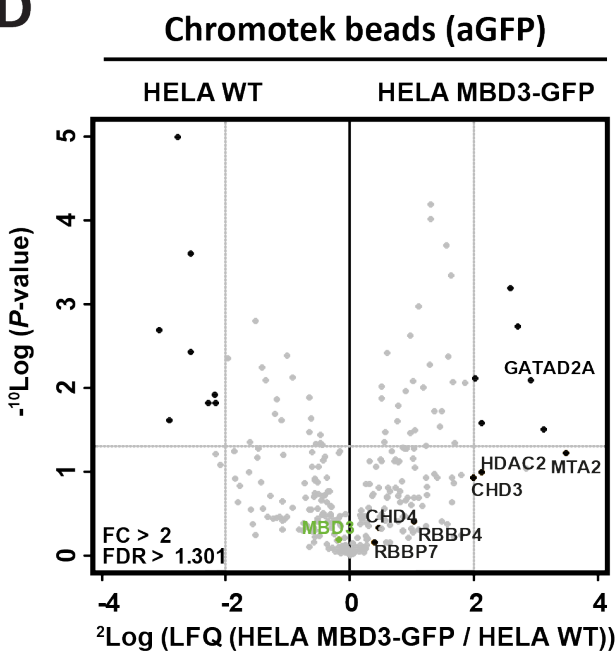

Figure 5.7 Mass spectrometry-based analysis of protein enrichment using non-modified, pSB-co(azido) ${ }_{8 \%}$, PSB-co-(aGFP) ${ }_{8 \%}$ and Chromotek beads represented as volcano plots. Statistically enriched proteins are identified using a FDR-corrected t-test. The ${ }^{-10} \log$-transformed P-values of the t-test (y-axis) are plotted against the relative label-free quantification (LFQ) intensities ( $x$-axis). Proteins are considered significant (black dots) with a ${ }^{2} \log$ fold-change $(\mathrm{FC})>2$ and a P-value $<0.05$, as indicated by the grey lines. A) Non-modified and PSB-co-(azido) ${ }_{8 \%}$ beads subjected to WT Hela whole cell lysate, B) pSB-co$(\mathrm{aGFP})_{8 \%}$ and Chromotek bead subjected to WT HeLa whole cell lysate, and C) pSB-co-(aGFP) ${ }_{8 \%}$ and D) Chromotek beads incubated in either WT HeLa nuclear extract or MBD3-GFP HeLa nuclear extract. 
nuclear extracts of WT HeLa cells (control samples). Under the conditions tested, only one relevant known interaction partner (GATAD2A) of MBD3 was identified with the Chromotek beads (see Figure 5.7D). The bait MBD3-GFP fusion protein as well some other proteins of the NuRD complex were only visible within the background, while at the same time five proteins were incorrectly identified as statistically relevant. Our pSB-CO-(aGFP) ${ }_{8 \%}$ beads on the other hand, were capable of enriching the MBD3-GFP protein (highlighted in green, Figure 5.7C) and identifying eight known interaction partners of MBD3. ${ }^{9,} 55-56$ Only one protein (presented as a red dot in Figure 5.7C), close to the FC $>2$ threshold, could not be identified as one of the MBD3 interaction partners and is therefore expected to be a non-specific binder. Noteworthy, the total number of detected proteins was much higher for the Chromotek beads than for the pSB-CO-(aGFP) ${ }_{8 \%}$ beads, whereas the amount of meaningful identified proteins, proteins from the NuRD complex, was considerable higher for the pSB-co- $(\mathrm{aGFP})_{8 \%}$ beads. As the number of beads, nuclear extract solutions and the reaction conditions were identical for both type of beads, and the amount of GFP captured in flow cytometry experiments was highly similar, the difference between them can be attributed to their difference in fouling. The fouling proteins do not only increase the risk of incorrectly identified protein-protein interactions but also hamper the identification of true interactors (as seen here for the Chromotek beads). These results stress the importance of reducing the non-specific binding of proteins within an IP-MS experiment. We have shown here that, in parallel with other strategies, antifouling beads can facilitate the identification of novel protein-protein interactions.

\subsection{Conclusion}

The use of antifouling antibody-coated beads that can be used within existing IP-MS procedures strongly reduces the amount of contaminating proteins, resulting in both a strong reduction in false positively identified protein-protein interactions and a more sensitive detection of true association partners. Such antifouling sulfobetaine-based polymer brushes were obtained by the copolymerization from the surface of microbeads of a standard sulfobetaine (SB) and an functionalizable azide-containing sulfobetaine (azido-SB), followed by the coupling of antiGFP nanobodies. The antifouling anti-GFP beads showed similar GFP binding efficiency but significantly decreased fouling as compared to commercially available Chromotek beads. As a result, within a co-IP-MS experiment, the antifouling beads displayed enrichment of the bait protein MBD3-GFP, together with seven other proteins which are known to operate in the same protein complex as MBD3, together with a strongly reduced amount of fouling. We hereby show the importance of reducing the non-specific binding of proteins within IP-MS experiments and demonstrate that the incorporation of antifouling coatings has great potential in diminishing contaminating proteins which can strongly facilitate the identification of true protein-protein interactions. 


\subsection{Acknowledgements}

The authors thank Sjef Boeren and Ben Meijer for technical assistance regarding nano LCMS/MS and flow cytometry measurements, respectively. Jorick Bruins and Floris van Delft are thanked for providing the Azide-Lissamine and for their helpful discussions. This work was supported by NanoNextNL (program 3E), a micro and nanotechnology consortium of the government of The Netherlands and 130 partners, to H.Z., and by an NWO-VICI grant to D.W.

\subsection{References}

1. Marsh, J. A.; Teichmann, S. A. Structure, Dynamics, Assembly, and Evolution of Protein Complexes. Annu. Rev. Biochem. 2015, $84,551-575$.

2. Huang, B. X.; Kim, H. Y. Effective Identification of Akt Interacting Proteins by Two-Step Chemical Crosslinking, CoImmunoprecipitation and Mass Spectrometry. PloS ONE 2013, 8, e61430.

3. Archakov, A. I.; Govorun, V. M.; Dubanov, A. V.; Ivanov, Y. D.; Veselovsky, A. V.; Lewi, P.; Janssen, P. Protein-Protein Interactions as a Target for Drugs in Proteomics. Proteomics 2003, 3, 380-391.

4. Fields, S.; Song, O. K. A Novel Genetic System to Detect Protein Protein Interactions. Nature 1989, 340, $245-246$.

5. Rajagopala, S. V.; Sikorski, P.; Caufield, J. H.; Tovchigrechko, A.; Uetz, P. Studying Protein Complexes by the Yeast TwoHybrid System. Methods 2012, 58, 392-399.

6. Mehmood, S.; Allison, T. M.; Robinson, C.V. Mass Spectrometry of Protein Complexes: From Origins to Applications. Annu. Rev. Phys. Chem. 2015, 66, 453-474.

7. Trinkle-Mulcahy, L.; Boulon, S.; Lam, Y.W.; Urcia, R.; Boisvert, F. M.; Vandermoere, F.; Morrice, N. A.; Swift, S.; Rothbauer, U.; Leonhardt, H.; Lamond, A. Identifying Specific Protein Interaction Partners Using Quantitative Mass Spectrometry and Bead Proteomes. J. Cell Biol. 2008, 183, 223-239.

8. Chan, J. S. K.; Teo, Z. Q.; Sng, M. K.; Tan, N. S. Probing for Protein-Protein Interactions During Cell Migration: Limitations and Challenges. Histol. Histopathol. 2014, 29, 965-976.

9. Smits, A. H.; Jansen, P.W.; Poser, I.; Hyman, A.A.;Vermeulen, M. Stoichiometry of Chromatin-Associated Protein Complexes Revealed by Label-Free Quantitative Mass Spectrometry-Based Proteomics. Nucleic Acids Res. 2013, 41, e28.

10. Mali, S.; Moree, W. J.; Mitchell, M.;Widger,W.; Bark, S. J. Observations on Different Resin Strategies for Affinity Purification Mass Spectrometry of a Tagged Protein. Anal. Biochem. 2016, 515, 26-32.

11. ten Have, S.; Boulon, S.; Ahmad, Y.; Lamond, A. I. Mass Spectrometry-Based Immuno-Precipitation Proteomics - the User's Guide. Proteomics 2011, 11, 1153-1159.

12. Dunham, W. H.; Mullin, M.; Gingras, A. C. Affinity-Purification Coupled to Mass Spectrometry: Basic Principles and Strategies. Proteomics 2012, 12, 1576-1590.

13. Ong, S. E.; Blagoev, B.; Kratchmarova, I.; Kristensen, D. B.; Steen, H.; Pandey, A.; Mann, M. Stable Isotope Labeling by Amino Acids in Cell Culture, SILAC, as a Simple and Accurate Approach to Expression Proteomics. Mol. Cell Proteomics 2002, 1, 376 386.

14. Boulon, S.; Ahmad, Y.; Trinkle-Mulcahy, L.; Verheggen, C.; Cobley, A.; Gregor, P.; Bertrand, E.; Whitehorn, M.; Lamond, A. I. Establishment of a Protein Frequency Library and Its Application in the Reliable Identification of Specific Protein Interaction Partners. Mol. Cell Proteomics 2010, 9, 861-879.

15. Meysman, P.; Titeca, K.; Eyckerman, S.; Tavernier, J.; Goethals, B.; Martens, L.; Valkenborg, D.; Laukens, K. Protein Complex Analysis: From Raw Protein Lists to Protein Interaction Networks. Mass Spectrom. Rev. 2017, 36, 600-614. 
16. Jiang, S. Y.; Cao, Z. Q. Ultralow-Fouling, Functionalizable, and Hydrolyzable Zwitterionic Materials and Their Derivatives for Biological Applications. Adv. Mater. 2010, 22, 920-932.

17. Schlenoff, J. B. Zwitteration: Coating Surfaces with Zwitterionic Functionality to Reduce Nonspecific Adsorption. Langmuir 2014, 30, 9625-9636.

18. Wei, Q.; Becherer,T.; Angioletti-Uberti, S.; Dzubiella, J.; Wischke, C.; Neffe, A. T.; Lendlein, A.; Ballauff, M.; Haag, R. Protein Interactions with Polymer Coatings and Biomaterials. Angew. Chem. Int. Ed. 2014, 53, 8004-8031.

19. Vaisocherová, H.; Brynda, E.; Homola, J. Functionalizable Low-Fouling Coatings for Label-Free Biosensing in Complex Biological Media: Advances and Applications. Anal. Bioanal. Chem. 2015, 407, 3927-3953.

20. Ostuni, E.; Chapman, R. G.; Holmlin, R. E.;Takayama, S.; Whitesides, G. M. A Survey of Structure-Property Relationships of Surfaces That Resist the Adsorption of Protein. Langmuir 2001, 17, 5605-5620.

21. Ladd, J.; Zhang, Z.; Chen, S.; Hower, J. C.; Jiang, S. Zwitterionic Polymers Exhibiting High Resistance to Nonspecific Protein Adsorption from Human Serum and Plasma. Biomacromolecules 2008, 9, 1357-1361.

22. Li, L. Y.; Chen, S. F.; Jiang, S. Y. Protein Interactions with Oligo(Ethylene Glycol) (Oeg) Self-Assembled Monolayers: Oeg Stability, Surface Packing Density and Protein Adsorption. J. Biomater. Sci., Polym. Ed. 2007, 18, 1415-1427.

23. Chen, S. F.; Li, L. Y.; Zhao, C.; Zheng, J. Surface Hydration: Principles and Applications toward Low-Fouling/Nonfouling Biomaterials. Polymer 2010, 51, 5283-5293.

24. Blaszykowski, C.; Sheikh, S.; Thompson, M. A Survey of State-of-the-Art Surface Chemistries to Minimize Fouling from Human and Animal Biofluids. Biomater. Sci. 2015, 3, 1335-1370.

25. Shao, Q.; Jiang, S. Y. Molecular Understanding and Design of Zwitterionic Materials. Adv. Mater. 2015, 27, 15-26.

26. Yang, W.; Chen, S. F.; Cheng, G.; Vaisocherova, H.; Xue, H.; Li, W.; Zhang, J. L.; Jiang, S. Y. Film Thickness Dependence of Protein Adsorption from Blood Serum and Plasma onto Poly(Sulfobetaine)-Grafted Surfaces. Langmuir 2008, 24, 9211 9214.

27. Huang, C. J.; Li, Y.; Krause, J. B.; Brault, N. D.; Jiang, S. Internal Architecture of Zwitterionic Polymer Brushes Regulates Nonfouling Properties. Macromol. Rapid Commun. 2012, 33, 1003-1007.

28. Lange, S. C.; van Andel, E.; Smulders, M. M. J.; Zuilhof, H. Efficient and Tunable Three-Dimensional Functionalization of Fully Zwitterionic Antifouling Surface Coatings. Langmuir 2016, 32, 10199-10205.

29. Brault, N. D.; White, A. D.; Taylor, A. D.; Yu, Q. M.; Jiang, S.Y. Directly Functionalizable Surface Platform for Protein Arrays in Undiluted Human Blood Plasma. Anal. Chem. 2013, 85, 1447-1453.

30. Chou,Y. N.;Wen,T. C.; Chang,Y. Zwitterionic Surface Grafting of Epoxylated Sulfobetaine Copolymers for the Development of Stealth Biomaterial Interfaces. Acta Biomater. 2016, 40, 78-91.

31. Hu, G. J.; Emrick, T. Functional Choline Phosphate Polymers. J.Am. Chem. Soc. 2016, 138, 1828-1831.

32. Li, Y.; Giesbers, M.; Gerth, M.; Zuilhof, H. Generic Top-Functionalization of Patterned Antifouling Zwitterionic Polymers on Indium Tin Oxide. Langmuir 2012, 28, 12509-12517.

33. Leslie, D. C.; Waterhouse, A.; Berthet, J. B.; Valentin, T. M.;Watters, A. L.; Jain, A.; Kim, P.; Hatton, B. D.; Nedder, A.; Donovan, K.; Super, E. H.; Howell, C.; Johnson, C. P.; Vu, T. L.; Bolgen, D. E.; Rifai, S.; Hansen, A. R.; Aizenberg, M.; Super, M.; Aizenberg, J.; Ingber, D. E. A Bioinspired Omniphobic Surface Coating on Medical Devices Prevents Thrombosis and Biofouling. Nat. Biotechnol. 2014, 32, 1134-40.

34. Moro, T.; Kyomoto, M.; Ishihara, K.; Saiga, K.; Hashimoto, M.; Tanaka, S.; Ito, H.; Tanaka, T.; Oshima, H.; Kawaguchi, H.; Takatori, Y. Grafting of Poly(2-Methacryloyloxyethyl Phosphorylcholine) on Polyethylene Liner in Artificial Hip Joints Reduces Production of Wear Particles. J. Mech. Behav. Biomed. Mater. 2014, 31, 100-106.

35. Kim, G.; Yong,Y.; Kang, H. J.; Park, K.; Kim, S. I.; Lee, M.; Huh, N. Zwitterionic Polymer-Coated Immunobeads for BloodBased Cancer Diagnostics. Biomaterials 2014, 35, 294-303.

36. Kirk, J. T.; Brault, N. D.; Baehr-Jones, T.; Hochberg, M.; Jiang, S.; Ratner, D. M. Zwitterionic Polymer-Modified Silicon Microring Resonators for Label-Free Biosensing in Undiluted Human Plasma. Biosens. Bioelectron. 2013, 42, 100-105. 
37. Mi, L.; Jiang, S. Integrated Antimicrobial and Nonfouling Zwitterionic Polymers. Angew. Chem. Int. Ed. Engl. 2014, 53, 17461754.

38. van Andel, E.; de Bus, I.; Tijhaar, E. J.; Smulders, M. M. J.; Savelkoul, H. F. J.; Zuilhof, H. Highly Specific Binding on Antifouling Zwitterionic Polymer-Coated Microbeads as Measured by Flow Cytometry. ACS Appl. Mater. Interfaces 2017, 9 , 38211-38221.

39. Rappsilber, J.; Mann, M.; Ishihama, Y. Protocol for Micro-Purification, Enrichment, Pre-Fractionation and Storage of Peptides for Proteomics Using Stagetips. Nat. Protoc. 2007, 2, 1896-1906.

40. Wendrich, J. R.; Boeren, S.; Möller, B. K.; Weijers, D.; De Rybel, B. In Vivo Identification of Plant Protein Complexes Using IP-MS/MS. In Plant Hormones: Methods and Protocols, Kleine-Vehn, J.; Sauer, M., Eds.; Springer New York: New York, 2017, pp 147-158.

41. Pyun, J.; Kowalewski, T.; Matyjaszewski, K. Synthesis of Polymer Brushes Using Atom Transfer Radical Polymerization. Macromol. Rapid Commun. 2003, 24, 1043-1059.

42. Matyjaszewski, K.; Xia, J. H. Atom Transfer Radical Polymerization. Chem. Rev. 2001, 101, 2921-2990.

43. Hong, V.; Presolski, S. I.; Ma, C.; Finn, M. G. Analysis and Optimization of Copper-Catalyzed Azide-Alkyne Cycloaddition for Bioconjugation. Angew. Chem. Int. Ed. 2009, 48, 9879-9883.

44. Debets, M. F.; Van Berkel, S. S.; Dommerholt, J.; Dirks, A. J.; Rutjes, F. P. J. T.; Van Delft, F. L. Bioconjugation with Strained Alkenes and Alkynes. Acc. Chem. Res. 2011, 44, 805-815.

45. Saenger, Y. M.; Li, Y.Y.; Chiou, K. C.; Chan, B.; Rizzuto, G.; Terzulli, S. L.; Merghoub, T.; Houghton, A. N.; Wolchok, J. D. Improved Tumor Immunity Using Anti-Tyrosinase Related Protein-1 Monoclonal Antibody Combined with DNA Vaccines in Murine Melanoma. Cancer Res. 2008, 68, 9884-9891.

46. Riedel, T.; Riedelova-Reicheltova, Z.; Majek, P.; Rodriguez-Emmenegger, C.; Houska, M.; Dyr, J. E.; Brynda, E. Complete Identification of Proteins Responsible for Human Blood Plasma Fouling on Poly(Ethylene Glycol)-Based Surfaces. Langmuir 2013, 29, 3388-3397.

47. Zimmer, M. Green Fluorescent Protein (GFP): Applications, Structure, and Related Photophysical Behavior. Chem. Rev. 2002, 102, 759-781.

48. Hubner, N. C.; Bird, A. W.; Cox, J.; Splettstoesser, B.; Bandilla, P.; Poser, I.; Hyman, A.; Mann, M. Quantitative Proteomics Combined with Bac Transgeneomics Reveals in Vivo Protein Interactions. J. Cell Biol. 2010, 189, 739-754.

49. Harikumar, A.; Edupuganti, R. R.; Sorek, M.; Azad, G. K.; Markoulaki, S.; Sehnalova, P.; Legartova, S.; Bartova, E.; FarkashAmar, S.; Jaenisch, R.; Alon, U.; Meshorer, E. An Endogenously Tagged Fluorescent Fusion Protein Library in Mouse Embryonic Stem Cells. Stem. Cell. Rep. 2017, 9, 1304-1314.

50. Galan, J. A.; Paris, L. L.; Zhang, H. J.; Adler, J.; Geahlen, R. L.; Tao, W. A. Proteomic Studies of Syk-Interacting Proteins Using a Novel Amine-Specific Isotope Tag and Gfp Nanotrap. J.Am. Soc. Mass Spectrom. 2011, 22, 319-328.

51. Wesolowski, J.; Alzogaray, V.; Reyelt, J.; Unger, M.; Juarez, K.; Urrutia, M.; Cauerhff, A.; Danquah, W.; Rissiek, B.; Scheuplein, F.; Schwarz, N.; Adriouch, S.; Boyer, O.; Seman, M.; Licea, A.; Serreze, D. V.; Goldbaum, F. A.; Haag, F.; Koch-Nolte, F. Single Domain Antibodies: Promising Experimental and Therapeutic Tools in Infection and Immunity. Med. Microbiol. Immun. 2009, 198, 157-174.

52. Gou, M.Y.; Huang, Q. S.; Qian, W. Q.; Zhang, Z. M.; Jia, Z. H.; Hua, J. Sumoylation E3 Ligase Siz1 Modulates Plant Immunity Partly through the Immune Receptor Gene Snc1 in Arabidopsis. Mol. Plant-Microbe Interact. 2017, 30, 334-342.

53. Surdo, N. C.; Berrera, M.; Koschinski, A.; Brescia, M.; Machado, M. R.; Carr, C.; Wright, P.; Gorelik, J.; Morotti, S.; Grandi, E.; Bers, D. M.; Pantano, S.; Zaccolo, M. FRET Biosensor Uncovers Camp Nano-Domains at Beta-Adrenergic Targets That Dictate Precise Tuning of Cardiac Contractility. Nat. Commun. 2017, 8.

54. Kittler, R.; Pelletier, L.; Ma, C.; Poser, I.; Fischer, S.; Hyman, A. A.; Buchholz, F. Rna Interference Rescue by Bacterial Artificial Chromosome Transgenesis in Mammalian Tissue Culture Cells. Proc. Natl.Acad. Sci. U. S.A. 2005, 102, 2396-401.

55. Menafra, R.; Stunnenberg, H. G. MBD2 and MBD3: Elusive Functions and Mechanisms. Front. Genet. 2014, 5. 
56. Kloet, S. L.; Baymaz, H. I.; Makowski, M.; Groenewold, V.; Jansen, P.W.T. C.; Berendsen, M.; Niazi, H.; Kops, G. J.; Vermeulen, M.Towards Elucidating the Stability, Dynamics and Architecture of the Nucleosome Remodeling and Deacetylase Complex by Using Quantitative Interaction Proteomics. FEBS J. 2015, 282, 1774-1785. 


\subsection{Supporting Information}

\subsubsection{Additional XPS spectra}
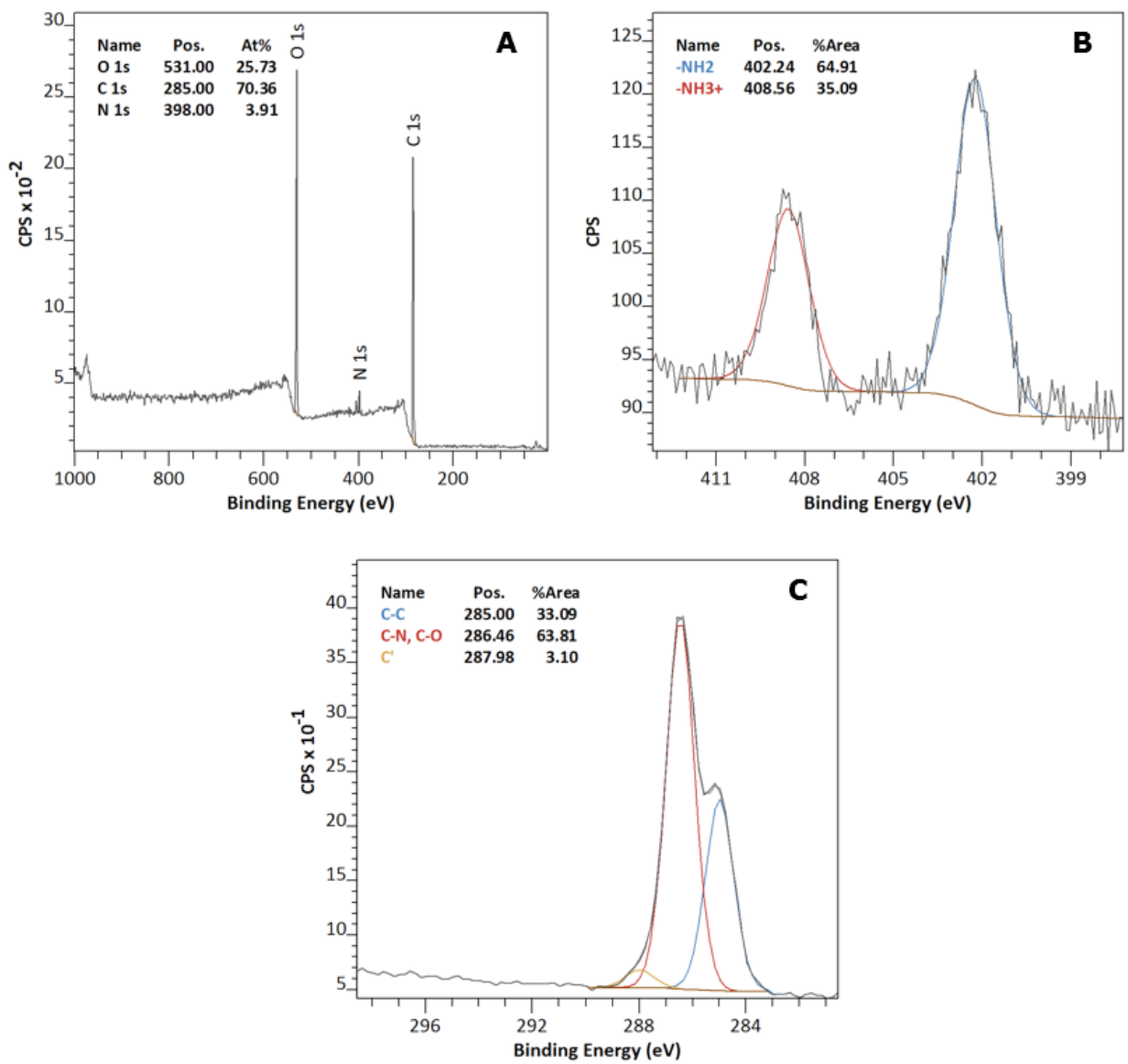

Figure S5.1 XPS spectra of non-modified Dynabeads: (A) wide scan and narrow scans of (B) N 1s and (C) C 1s. C' probably originates from a $\mathrm{C}-\mathrm{O}$ or $\mathrm{C}-\mathrm{N}$ species. 

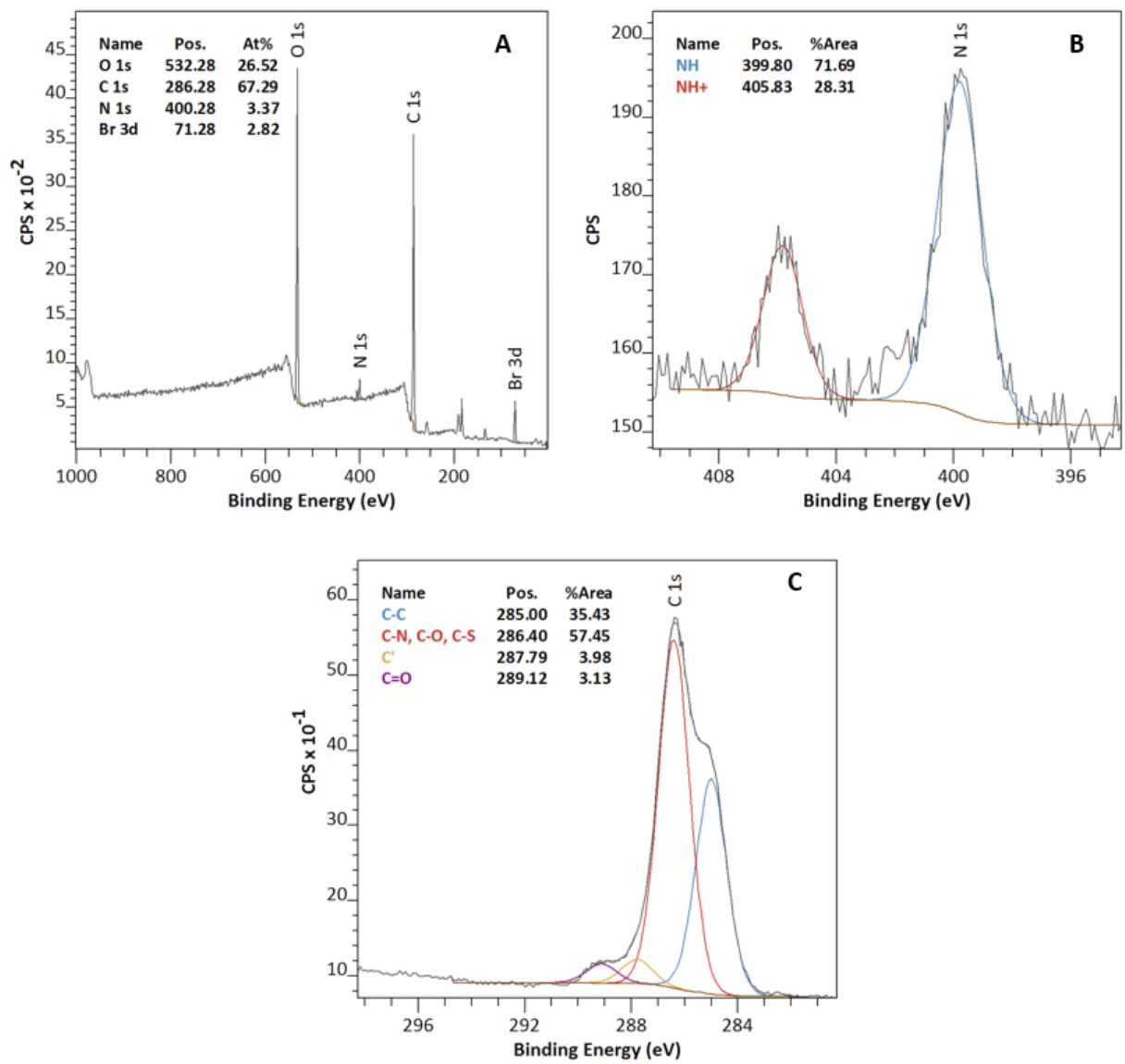

Figure S5.2 XPS spectra of Dynabeads functionalized with $\alpha$-bromoisobutyryl bromide: (A) wide scan and narrow scans of (B) N 1s and (C) C 1s. The peak labeled as C' originates from the bare Dynabead (see Figure S5.1). 

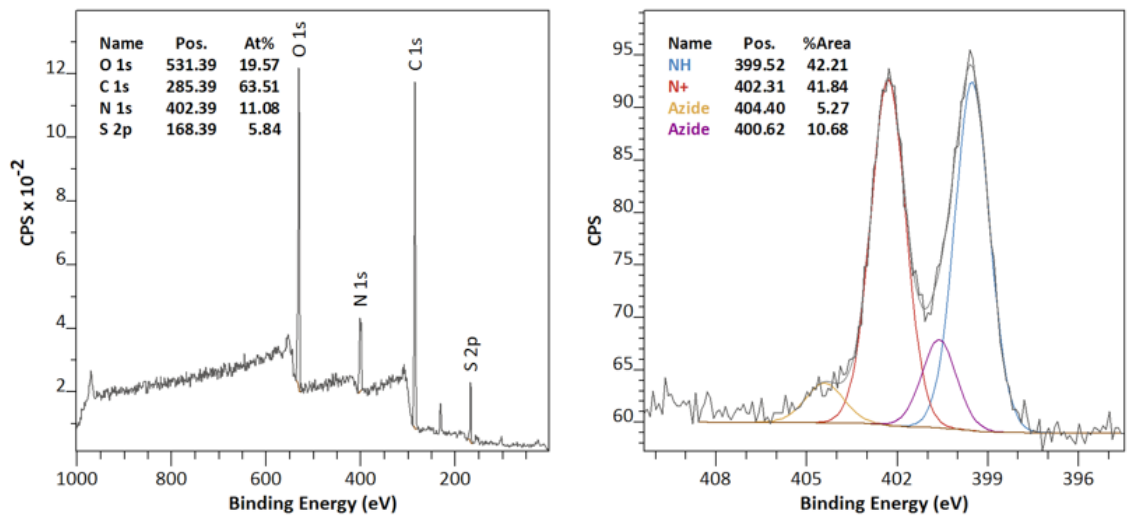

Figure S5.3 XPS wide scans (left) and XPS N 1s narrow scans (right) of pSB-co-(azido) ${ }_{16 \%}$ beads. The spectra show the successful growth of zwitterionic copolymer brushes of SB and azido-SB monomer from beads as seen by the appearance of sulfur signals in the wide scans, the $1: 1$ ratio of ammonium versus amide nitrogen peak and the appearance of $\mathrm{N} 1 \mathrm{~s}$ azide peaks.

\subsubsection{Obtaining homogeneous bead samples}

In order to obtain homogeneous samples, i.e. sharp peaks by flow cytometry, it is essential to keep the beads in suspension during all steps as the beads settle quickly. In the first step, where amine-terminated Dynabeads are reacted with $\alpha$-bromoisobutyryl bromide, this is achieved by placing the reaction tube on an end-over-end shaker. During ATRP, the homogeneity of the samples is maintained by shaking on an incubator shaker at $80 \mathrm{rpm}$. We used a wide flask (diameter of $\sim 3.5 \mathrm{~cm}$ ) with a round shaped bottom under an angle of approximately $45^{\circ}$ to create a large surface area (if the tube that is used is too narrow the beads will settle despite the shaking). All functionalization, staining and protein incubation steps were performed in separate PCR tubes which were taped on an end-over-end shaker ( $4 \mathrm{rpm}$ ) of which the rotating wheel was placed exactly perpendicular to the table. If the rotating wheel is placed under an angle the beads will settle against the wall of the tube, which should be avoided. The smaller the volume the more challenging it becomes to keep all beads in suspension. The narrow PCR tubes ensure that the suspension will stay at the bottom of the PCR tube, gravity together with rotation will keep the beads in suspension. Volumes ranging from 20-150 $\mu \mathrm{L}$ can be reliably used when using the PCR tubes. When larger volumes are desired, $2 \mathrm{~mL}$ Eppendorf tubes can be reliably used with volumes $\geq 200 \mu \mathrm{L}$. In this case the exact placement of the tube on the end-over-end shaker is less crucial as the entire bead volume will be 'rolling' through the tube (for this reason $1.5 \mathrm{~mL}$ Eppendorf tubes together with small volumes are less suitable as part of the sample will be retained at the bottom of the tube and part is likely to get stuck in the lid, leading to inhomogeneous samples). We used a maximum of $2 \times 10^{6}$ beads per $\mu \mathrm{L}$ of sample. It is also recommended to quickly collect all beads after each incubation step by a pulse spin of a few seconds in an Eppendorf centrifuge. 


\subsubsection{Full size SDS-PAGE gels}

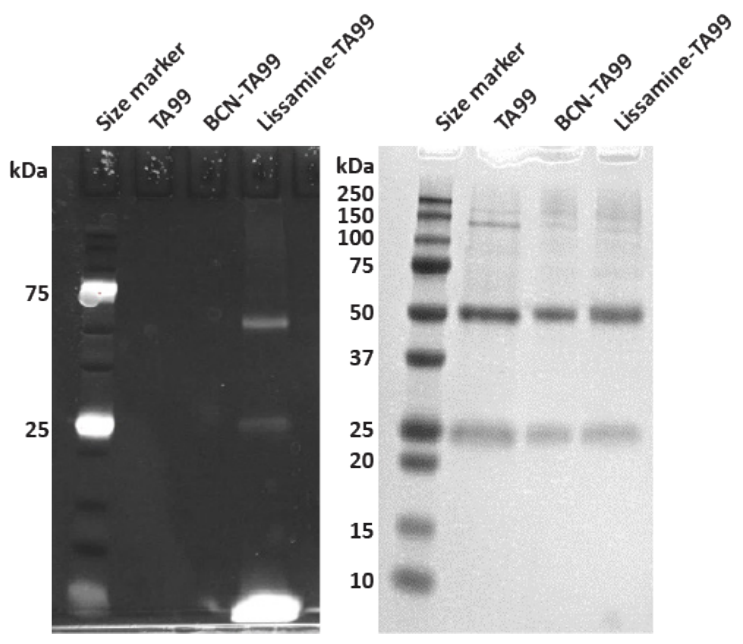

Figure S5.4 Full size SDS-PAGE gel of untreated TA99 antibody, TA99 labeled with BCN-NHS (BCNTA99), and BCN-TA99 reacted with Azide-Lissamine (Liss-TA99). Visualization by fluorescence (left) using Bio-Rad ChemiDoc ${ }^{\mathrm{TM}}$ XRS+ with standard 580 AF 120 filter followed by Coomassie blue staining (right). The very bright band at low molecular weight corresponds to free Azide-Lissamine.

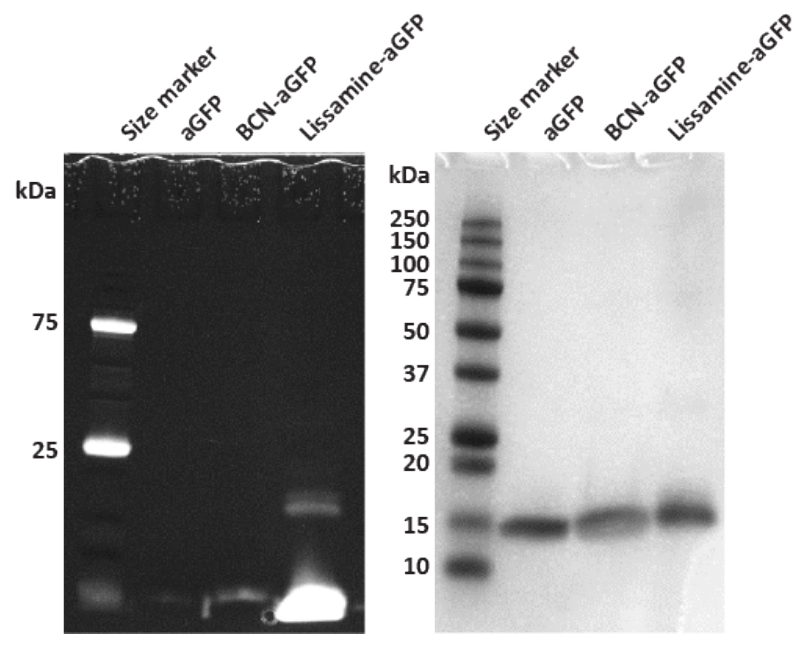

Figure S5.5 Full size SDS-PAGE gel of untreated aGFP antibody, aGFP labeled with BCN-NHS (BCNaGFP), and BCN-aGFP reacted with Azide-Lissamine (Lissamine-aGFP). Visualization by fluorescence (left) using Bio-Rad ChemiDoc ${ }^{\mathrm{TM}}$ XRS+ with standard 580 AF 120 filter followed by Coomassie blue staining (right). The very bright band at low molecular weight corresponds to free Azide-Lissamine. 


\subsubsection{Additional flow cytometry data}

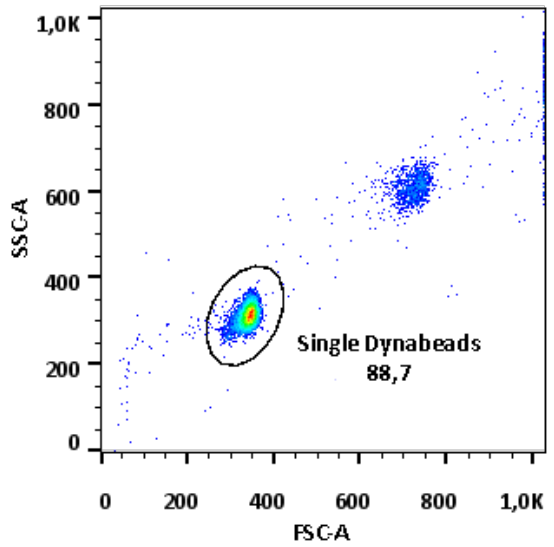

Figure S5.6 Typical side/forward scatter plot of Dynabeads as obtained by flow cytometry. A total of 10.000 single beads were acquired per sample and used in further analysis. This type of gating was used for Figure 5.3, Figure 5.4, Figure S5.7 and Figure S5.8.

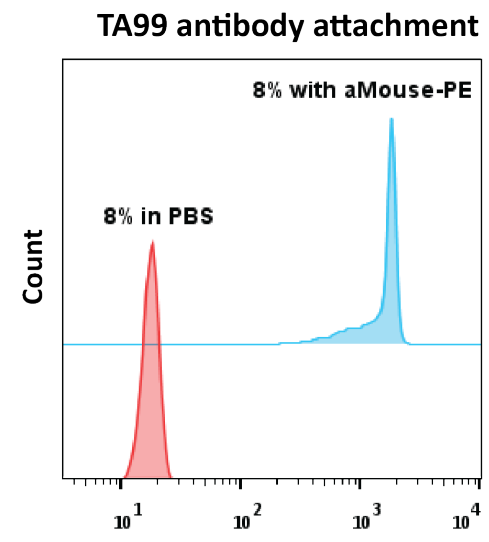

Fluorescence intensity (PE)

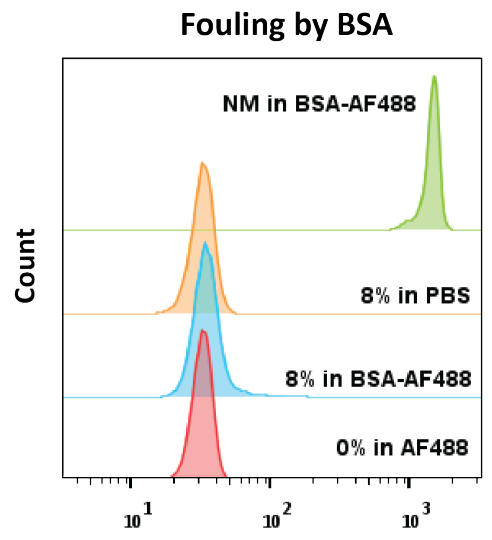

Fluorescence intensity (FITC)

Figure S5.7 Typical flow cytometry data of pSB-co-(TA99) ${ }_{8 \%}$ beads. In the PE channel (left), pSB-co(TA99 $_{8 \%}$ beads in either PBS or stained with an anti-Mouse-PE antibody are shown. The FITC channel (right) shows pSB-co-(TA99) ${ }_{8 \%}$ beads in PBS and incubated with BSA-AF488, as well as pSB and nonmodified (NM) beads incubated with BSA-AF488. This data confirms the attachment of TA99 by the fluorescence increase of anti-Mouse-PE stained beads and the resistance of these beads against the nonspecific binding of BSA-AF488 to these beads. 

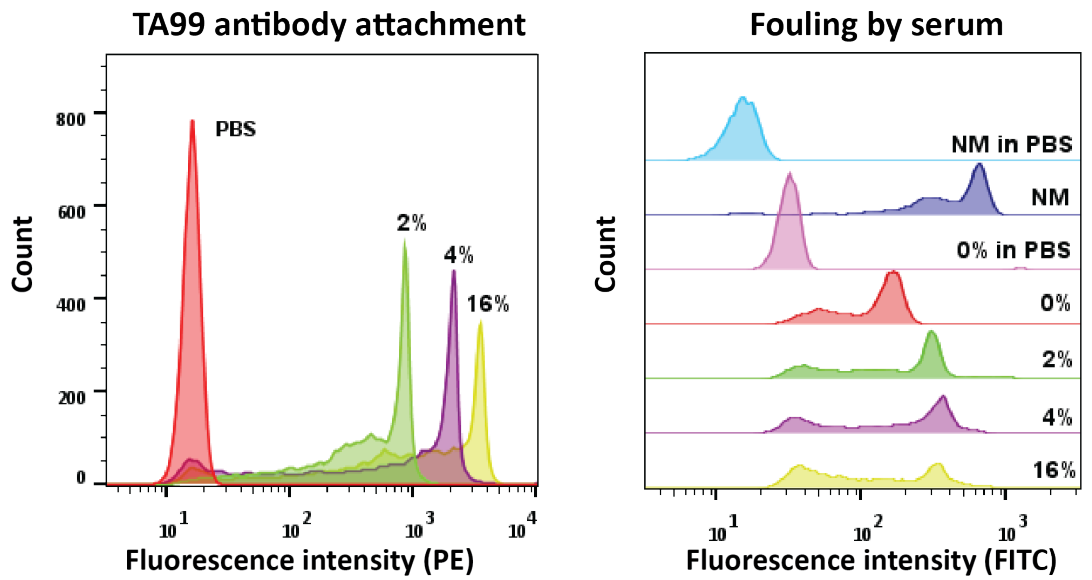

Figure S5.8 Flow cytometry data of pSB-CO-(TA99) ${ }_{\mathrm{m} \%}$ beads. In PE channel (left), pSB-co-(TA99) m\% $_{\mathrm{m} \%}$ beads in PBS and with anti-Mouse-PE antibody staining are shown $(\mathrm{m}=2,4$, and $16 \%)$. The FITC channel (right) shows pSB-co-(TA99) ${ }_{0 \%}$ and non-modified (NM) beads in PBS, and NM and pSB-co-(TA99) $)_{\mathrm{m} \%}(\mathrm{~m}$ $=0,2,4,16$ ) beads incubated with Serum-Biotin followed by Strep-FITC. This data shows the increased TA99 attachment with increasing azido percentages. Increased fouling is observed for increased TA99 attachment, although the amount of fouling is still significantly lower than for NM beads.

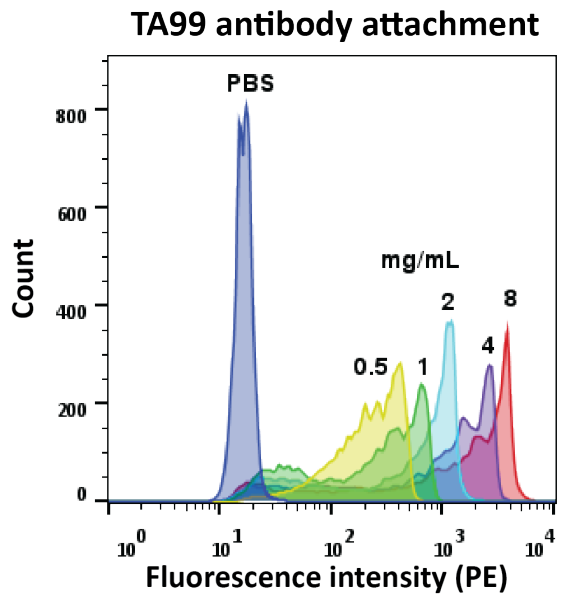

Figure S5.9 Flow cytometry data of pSB-CO-(TA99) ${ }_{8 \%}$ beads. pSB-CO-(TA99) ${ }_{8 \%}$ beads in PBS are shown together with pSB-CO-(TA99) ${ }_{8 \%}$ beads prepared by using $0.5,1,2,4$ or $8 \mathrm{mg} / \mathrm{mL}$ TA99. Beads were stained using an anti-Mouse-PE antibody. This data shows the increased TA99 attachment with increasing TA99 concentration in the preparation of pSB-CO-(TA99) ${ }_{8 \%}$ beads. 

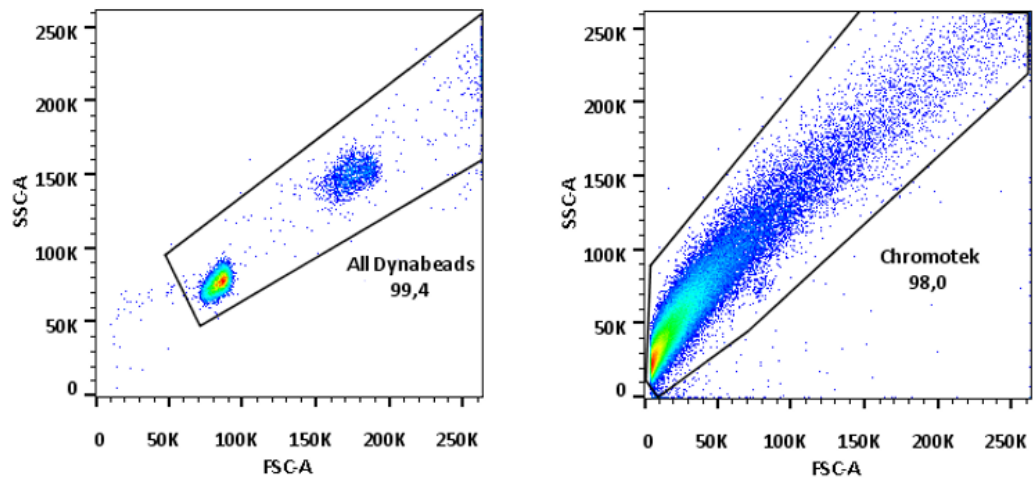

Figure S5.10 Example of flow cytometry gating for the comparison of Dynabeads and Chromotek beads. All bead events are selected, 10.000 bead events were measured per sample. This type of gating was used for Figure 5.5.

\subsubsection{Additional nano LC-MS/MS data}

A

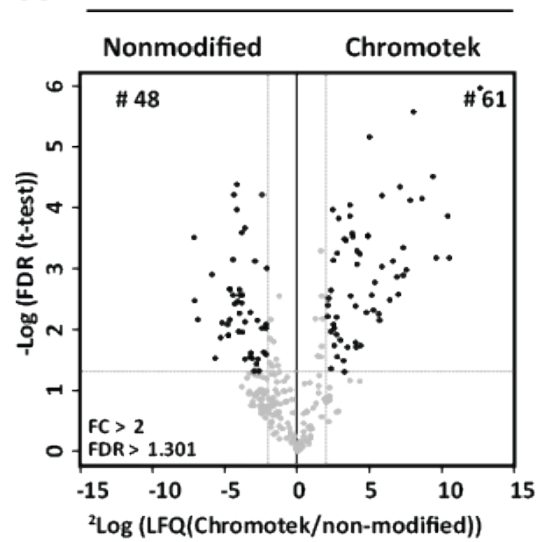

B HELA WT

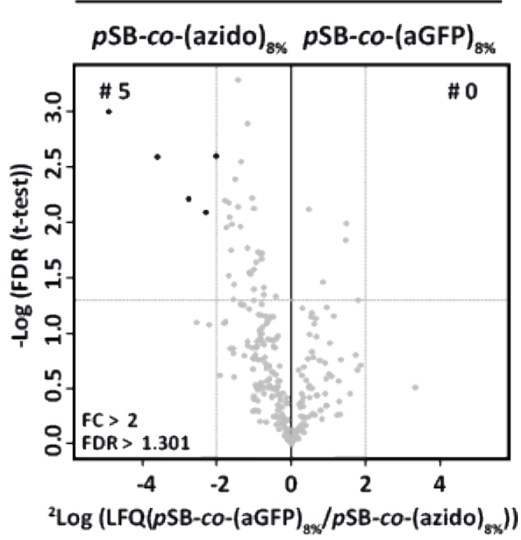

Figure S5.11 Mass spectrometry-based analysis of protein enrichment using non-modified, pSB-co(azido) ${ }_{8 \%}$, PSB-Co-(aGFP) ${ }_{8 \%}$ and Chromotek beads represented as volcano plots. Statistically enriched proteins are identified using a FDR-corrected t-test. The relative label-free quantification (LFQ) intensities (x-axis) are plotted against the ${ }^{-10} \log$-transformed $P$-values of the t-test ( $y$-axis). A) Non-modified and Chromotek beads, and B) pSB-co-(azido $)_{8 \%}$ and pSB-co-(aGFP) ${ }_{8 \%}$ beads, subjected to WT HeLa whole cell lysate. 


\section{Chapter.}




\section{Targeted \\ Phagocytosis of Functionalized}

\section{Stealth Microbeads by}

Macrophages

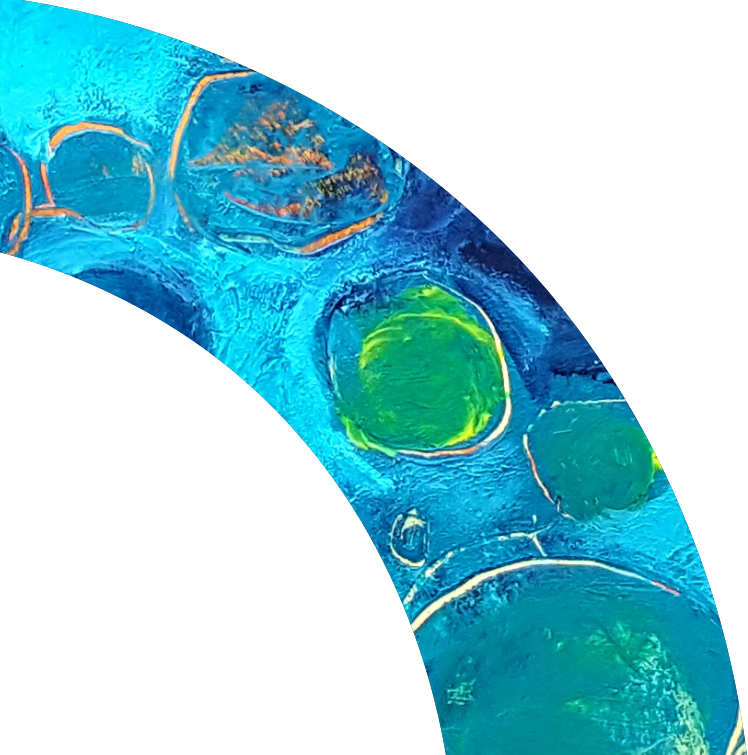

van Andel, E.; Cuenca-Escalona, J.; van der Zanden, S.; Smulders, M. M. J.; Zuilhof, H.; Savelkoul, H. F. J.; Tijhaar, E. J. Targeted Phagocytosis of Functionalized Stealth Microbeads by Macrophages. Manuscript in preparation. 


\begin{abstract}
Particles (nano- to micron-sized) represent highly promising platforms for drug delivery, particle-based vaccines and in vivo imaging. One of the major obstacles to fulfill the potential of these particles is the formation of a protein corona by non-specific adsorption of proteins onto the particle surface. The formation of a protein corona promotes not only adverse effects such as clearance from the blood stream by cells from the mononuclear phagocytic system, like macrophages, but also inflammation and uncontrolled localization. Poly(ethylene glycol) (PEG) has been used as antifouling material to prevent protein adsorption onto particles. To overcome the limitations of PEG-based coatings in terms of antifouling performance and immunogenicity, zwitterionic polymer brushes grown from a surface have been shown to have excellent antifouling properties and attachment of such coatings onto the particles is therefore of high interest to advance the use of these particles in biomedical applications. In this study, we show that such a zwitterionic coating strongly reduces fouling and creates 'stealth' beads that remain nearly invisible for macrophages, resulting in strongly reduced phagocytosis of these beads. Immobilization of ovalbumin on these antifouling beads did not affect their stealth characteristics. However, when the beads were specifically targeted to the Fc gamma receptor on macrophages by binding of ovalbumin-specific antibodies of the $\mathrm{IgG}_{2 \mathrm{a}}$ isotype, they were efficiently phagocytosed. These results show the high potential of functionalized zwitterionic polymer brushes for in vivo particle-based applications.
\end{abstract}




\subsection{Introduction}

Spatially and temporally controlled drug delivery strategies are in high demand, as controlled release of drugs at specific therapeutic sites can significantly lower the systemic drug dose and prevent damage to healthy tissues. This contributes to a better disease treatment and quality of life of treated patients. ${ }^{1}$ A new area in this field is the development of theranostics: nanotherapeutic systems that can simultaneously diagnose cancer, deliver therapy and monitor the effect of the therapy. ${ }^{2-4}$ Theranostics often rely on nanoparticles that can both be used for imaging as well as for drug delivery. ${ }^{2,4}$ The major obstacle to fulfill the potential of these multi-functional particles is their rapid clearance from the blood stream by the mononuclear phagocytic system (MPS). ${ }^{1}$ Macrophages of the MPS have the ability to remove the nanoparticles from the blood stream within seconds after intravenous administration, preventing the therapeutic agents to reach their target site. ${ }^{5-6}$ The macrophages typically recognize the particles by so-called opsonins that become non-specifically bound to the particle upon in vivo administration, and which are often referred to as a protein corona. ${ }^{7}$ Opsonins are those blood serum proteins that aid in phagocytic recognition, such as complement proteins C3-5 and immunoglobulins. ${ }^{1,8}$ The formation of a protein corona does not only prevent a drug carrier to reach its target site, but can also cause the accumulation of carriers in tissues of the MPS, resulting in inflammation, release of toxic products as well as to uncontrolled localization. ${ }^{9}$ It is evident that prevention of non-specific adsorption of opsonins to theranostic particles is highly desired.

An often employed strategy to avoid the formation of a protein corona is through the incorporation of protein-repellent (also referred to as antifouling) materials. To achieve this, poly(ethylene glycol) (PEG) is most commonly utilized. ${ }^{3,10-11}$ Despite the frequent use of PEGylated particles, PEG has various shortcomings. The protein-repellent capacity of PEG materials in body fluids is limited, ${ }^{12}$ PEG is prone to oxidative damage ${ }^{13-14}$ and enzymatic degradation of PEG molecules has been shown to produce toxic metabolites upon in vivo administration. ${ }^{15-16}$ In addition, anti-PEG antibodies have been detected on several occasions in response to injected PEGylated particles. ${ }^{16-17}$ The formation of anti-PEG antibodies has adverse effects on PEGylated drug carriers as it leads to fast clearance of the drug vehicles due to antibody-mediated phagocytosis. These limitations have shifted the development towards zwitterionic particles. ${ }^{9}$ Zwitterions are defined as molecules that bear both positive and negative charges, but are overall charge neutral. Zwitterionic polymer brush coatings have been shown to have excellent antifouling properties, which can be attributed to a layer of tightly bound water molecules, also referred to as a hydration layer, that prevents proteins from adsorbing to the zwitterionic coating. ${ }^{18-19}$ On flat surfaces, it has been shown that the best zwitterionic antifouling coatings are prepared by the grafting from approach using Surface-Initiated Atom Transfer Polymerization (SI-ATRP), in which polymers are grown from the surface (see chapter 2). ${ }^{18,20-21}$ This method yields polymer brush coatings of high density with excellent antifouling properties. ${ }^{22}$ This method has, however, hardly been used on solid particles. Studies that utilize zwitterionic polymer brushcoated particles are typically used for other applications than avoiding phagocytic clearance, such as improved stability of immobilized enzymes ${ }^{23}$ or antimicrobial activity. ${ }^{24}$ To the best of our knowledge, only one study by Jiang et al., ${ }^{25}$ reported the use of zwitterionic polymer brush- 
coated nanoparticles to avoid phagocytic uptake. However, functionalization and targeting of these particles to specific cell types was not part of this study.

Building on the work described in chapters 3-5 on zwitterionic polymer brush-coated beads, here we show a proof-of-principle study with the antifouling microbeads prepared via SIATRP that can effectively prevent phagocytosis by murine macrophages. Fluorescent beads were used to enable quantification of their internalization by flow cytometry and confocal microscopy. The incorporation of functional zwitterionic monomers allowed for the effective modification of the brushes with proteins and antibodies. Functionalization with the model protein ovalbumin (OVA), did not lead to an increase in phagocytosis, illustrating the stealth character of the zwitterionic polymer brush-coated beads. In addition, we show the targeting of antigen-presenting cells via the direct and indirect labeling of $\operatorname{IgG}_{2 a}$ antibodies onto the antifouling beads, leading to Fc gamma receptor-mediated phagocytosis. This shows the high potential of antifouling ZPB-coated particles, functionalized with specific recognition elements for the targeting of specific cells.

\subsection{Materials and methods}

\subsubsection{Materials}

All chemicals and solvents were used without further purification. Anhydrous dimethylsulfoxide (DMSO), 2-bromoisobutanoic acid N-hydroxysuccinimide ester, bovine serum albumin (BSA), albumin (OVA) chicken egg grade VII, and monoclonal anti-chicken egg albumin antibody

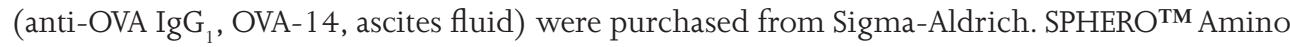
Fluorescent Pink Magnetic Particles (Spherotech beads) were obtained from Spherotech, Dynabeads M-270 amine from Invitrogen Life Technologies, fetal calf serum (FCS) from Gibco, and Roswell Park Memorial Institute (RPMI) medium from Lonza. DRAQ7 was purchased from BioStatus, streptavidin-phycoerythrin (Strep-PE) conjugate from eBioscience, bovine serum albumin-Alexa Fluor 488 conjugate (BSA-AF488) from Thermo Fisher, and endo-BCN-PEG NHS ester (BCN-NHS) from tebu-bio. Phycoerythrin (PE) conjugated goat anti-mouse IgG antibody (aMouse-PE, clone: Poly4053, $0.2 \mathrm{mg} / \mathrm{mL}$ ) and anti-chicken ovalbumin antibody (anti-OVA IgG ${ }_{2 a}$, clone TOSGAA1, $0.5 \mathrm{mg} / \mathrm{mL}$ ) were bought from BioLegend. MilliQ was produced with a MilliQ Integral 3 system (Millipore).

\subsubsection{Synthesis}

The syntheses of 3-((3-methacrylamidopropyl)dimethylammonio)propane-1-sulfonate (SB3) monomer and 3-((3-azidopropyl)(3-methacrylamidopropyl) (methyl)ammonio)propane-1sulfonate (azido-SB) monomer were performed as previously described (chapter 2). ${ }^{21}$ 


\subsubsection{Initiator attachment}

Amine-terminated Dynabeads were reacted with $\alpha$-bromoisobutyryl bromide as previously described (chapter 5). Initiator attachment on amine-terminated Spherotech beads was performed as follows: $300 \mu \mathrm{L}$ of Spherotech bead slurry was washed twice with $500 \mu \mathrm{L}$ MilliQ and after removal of all the all MilliQ the beads were dried in a vacuum oven (15 mbar) at $50{ }^{\circ} \mathrm{C}$ for $2 \mathrm{~h}$. Within a glovebox, a $50 \mathrm{mM}$ solution of 2-bromoisobutanoic acid $\mathrm{N}$-hydroxysuccinimide ester in anhydrous DMSO was prepared. Outside of the glovebox, the dried beads were resuspended in $500 \mu \mathrm{L}$ of this solution and incubated on an end-over-end shaker for $3 \mathrm{~h}$ at RT. Thereafter, the beads were washed twice with DMSO and twice with MilliQ. The beads were immediately used for further modification.

\subsubsection{Surface-initiated polymerization}

Surface-Initiated Atom Transfer Radical Polymerization (SI-ATRP) on initiator-equipped Dynabeads and Spherotech beads using SB monomer to yield pSB beads, was performed as previously described (chapter 4), with the modification that brushes were grown for 15 min. The copolymerization of SB and azido-SB to yield pSB-co-(azide) ${ }_{8 \%}$ Dynabeads was performed as described in chapter 5 .

\subsubsection{X-Ray Photoelectron Spectroscopy (XPS)}

Prior to XPS analysis, modified beads (in MilliQ) were dropcasted on a plasma-cleaned piece of $\operatorname{Si}(111)$ and dried in a vacuum oven at $50{ }^{\circ} \mathrm{C}$ for at least $2 \mathrm{~h}$. The beads were subsequently analyzed using a JPS-9200 photoelectron spectrometer (JEOL, Japan). The spectra were obtained using monochromatic $\mathrm{Al} \mathrm{K \alpha} \mathrm{X}$-Ray radiation at $12 \mathrm{kV}$ and $20 \mathrm{~mA}$, with an analyzer energy pass of $10 \mathrm{eV}$ for narrow scans. The obtained spectra were processed using CASA XPS peak fit program (version 2.3.16 PR 1.6).

\subsubsection{OVA functionalization, characterization and immobilization on beads}

Ovalbumin (OVA) labeling with endo-BCN-PEG - NHS ester (BCN-NHS) and subsequent characterization, using Azide-Lissamine and SDS-PAGE, and immobilization on pSB-CO(azide) ${ }_{8 \%}$ Dynabeads was performed as described in chapter 5.

\subsubsection{Cell culturing and phagocytosis assay}




\section{CELLS}

RAW 264.7 cells (ATCC) were grown using RPMI medium supplemented with 10\% FCS, 2 mM L-glutamine, $100 \mu \mathrm{g} / \mathrm{mL}$ penicillin and $100 \mu \mathrm{g} / \mathrm{mL}$ streptomycin, at $37{ }^{\circ} \mathrm{C}$ and $5 \% \mathrm{CO}_{2}$. Cells were passaged twice a week at 80-90\% confluency.

\section{PHAGOCYTOSIS}

The RAW cells were harvested using a cell scraper, centrifuged for 5 min at RT, resuspended in fresh medium and counted using a Bürker counting chamber. The cells were seeded in a 12 -wells plate with $0.5 \times 10^{6}$ cells per well in $1 \mathrm{~mL}$ of medium. The cells were allowed to recover for $60 \mathrm{~min}$ in an incubator $\left(37^{\circ} \mathrm{C}\right.$ and $\left.5 \% \mathrm{CO}_{2}\right)$. After that, the medium was refreshed and the beads were added to the cells in a $1: 10$ cells to beads ratio. Beads and cells were incubated together for $4 \mathrm{~h}$ in the incubator. The cells were then analyzed by light microscopy followed by flow cytometry analysis.

\subsubsection{Microscopy}

\section{LIGHT MICROSCOPY}

Pictures of cells (within the 12-wells plate) were made using an EVOS FL Cell Imaging system (Thermo Fisher Scientific).

\section{CONFOCAL MICROSCOPY}

For confocal microscopy imaging, the phagocytosis assay was performed in an ibiTreat $\mu$-Slide 8 Well chambered coverslip (purchased from ibidi). Per well, $2 \times 10^{5}$ cells were seeded which were allowed to recover overnight in an incubator (incubated at $37{ }^{\circ} \mathrm{C}$ and $5 \% \mathrm{CO}_{2}$ ) The next day, the medium was refreshed and the cells were incubated with $1 \times 10^{6}$ nonmodified (NMS-S) or pSB-coated Spherotech beads (pSB-S) in a total volume of $250 \mu \mathrm{L}$. Cells and beads were incubated together for $4 \mathrm{~h}$ within the incubator. The cells were stained with carboxyfluorescein succinimidyl ester (CFSE) based on a procedure described by Piazzon et al. ${ }^{26}$ The entire staining procedure was performed within the chambered coverslip. Cells were 3 times carefully washed with $250 \mu \mathrm{L}$ of $0.1 \%$ BSA/PBS (RT). To each well, $125 \mu \mathrm{L}$ of $0.1 \% \mathrm{BSA} /$ PBS was added, and supplemented with $125 \mu \mathrm{L}$ of CFSE solution to a final concentration of 18 $\mu \mathrm{M}$ CFSE. The cells were stained in the incubator for exactly $10 \mathrm{~min}$. The staining solution was replaced with cold medium and the cells were incubated on ice for $5 \mathrm{~min}$. The cells were 3 times washed with ice-cold PBS, and were subsequently analyzed in PBS using a confocal laser scanning microscope (Leica TCS SP8X system), equipped with a $63 \times / 1$.2NA water immersion objective. CFSE and Spherotech beads were excited using a white-light pulsed (repetition rate $40 \mathrm{MHz}$ ) laser selecting the 488-laser line. Fluorescence emission was collected between 500 and $531 \mathrm{~nm}$ for CFSE, and the emission of the Spherotech beads between 570 and $610 \mathrm{~nm}$. Confocal images were analyzed with the LAS AF Lite (version 2.6.0) software. 


\subsubsection{Flow cytometry}

\section{BEADS}

The antifouling performance of the beads was evaluated by incubating $2 \times 10^{6}$ beads in either PBS, BSA-AF488 $(0.5 \mathrm{mg} / \mathrm{mL})$ or $10 \%$ biotinylated bovine serum solution, both diluted in PBS, as described in chapters 4 and 5. Immobilization of OVA on pSB-CO-(azide) ${ }_{8 \%}$-D beads was evaluated by incubating the beads with anti-OVA $\operatorname{IgG}_{2 \mathrm{a}}$ (1:50 dilution), followed by antiMouse-PE (1: 50 dilution).

\section{CELLS}

After the phagocytosis assay, cells and beads were collected from the 12 -wells plate using a cell-scraper, $0.5 \mathrm{~mL}$ of the obtained suspension was directly transferred to a FACS tube. To each sample, viability dye DRAQ7 was added (1: 4000 dilution). The samples were first gated on single live cells (10,000 events) before further analysis on fluorescence and SSC/FSC.

Cells and beads were analyzed with a BD FACS Canto A (BD Biosciences) flow cytometer. BSAAF488 was visualized in the FITC-channel, Strep-PE, anti-Mouse-PE and Spherotech beads in the PE-channel, and DRAQ7 in the APC-Cy7-A channel.

\subsection{Results and Discussion}

\subsubsection{Phagocytosis of Spherotech beads by RAW cells}

To be able to study the phagocytic uptake of beads by confocal microscopy and flow cytometry, fluorescent magnetic amine-terminated Spherotech beads were selected. The Spherotech beads were coated with zwitterionic polymer brushes via SI-ATRP using a standard sulfobetaine (SB) monomer. The successful brush formation was confirmed by X-ray Photoelectron Spectroscopy (XPS) (for XPS spectra and discussion on the results, see the Supporting Information). RAW cells, from a murine macrophage cell line, were used to study the phagocytosis of Spherotech beads with (pSB-S) and without (NM-S) antifouling coating (see Figure 6.1). To this end, RAW cells were incubated with either pSB-S beads or NM-S beads. Initial studies by light microscopy imaging (see Figure 6.2) showed clear phagocytosis of the NM-S by the RAW cells: numerous beads per cell were internalized. In fact, in some of the cells so many beads were taken up that the position of the cell nuclei became visible. In contrast, the pSB-S beads were only scarcely taken up by the macrophages, showing that the pSB-S beads had stealth characteristics that made them nearly undetectable for the RAW cells.

RAW cells incubated with NM-S were also studied using confocal microscopy, see Figure 6.3. The cells were visualized using CFSE staining (in green), while the fluorescent NM-S beads are 


\section{Spherotech beads (S)}

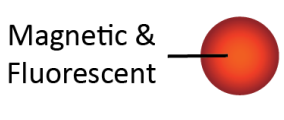

NM-S

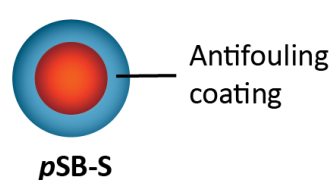

pSB-S

\section{Dynabeads (D)}
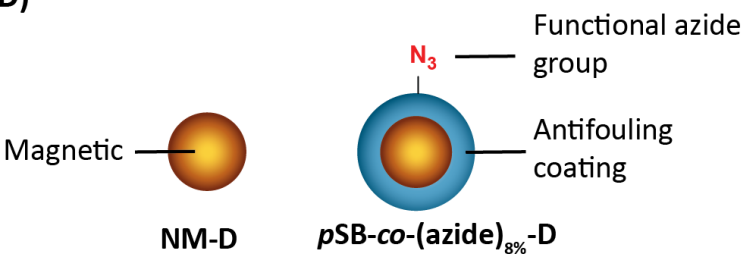

\section{Functionalized Dynabeads (D)}
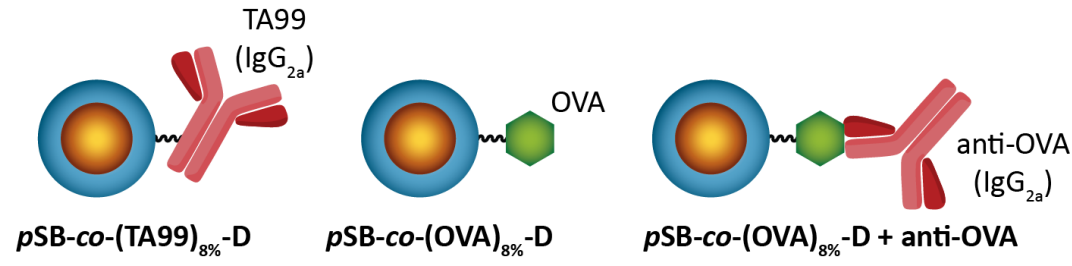

Figure 6.1 Overview of beads used: Spherotech and Dynabeads either non-modified (NM) or coated with a covalently grown zwitterionic antifouling coating (pSB). Antifouling Dynabeads were also equipped with functional azide moieties (pSB-co-(azide) ${ }_{8 \%}$ beads). These beads were further modified by the immobilization of TA99 antibody, ovalbumin (OVA) or first by OVA and then by an anti-OVA antibody.

shown in red. The CFSE panel shows two cells, both with areas where the CFSE staining is absent. In the overlay panel it can be seen that the beads are located in those areas. This confirms that the beads were indeed internalized by the macrophages and not just attached to the outside of the cells. An overview picture of a large number of cells is given in the Supporting Information Figure S6.3. The great majority of beads were internalized; occasionally a bead was observed at the outside of a cell.

To quantify the difference in phagocytosis between the beads with and without the antifouling layer, the cells were analyzed by flow cytometry (Figure 6.4). Three different samples were compared: RAW cells only, to which no beads had been added, and RAW cells incubated with either NM-S or pSB-S beads. Figure 6.4 displays single live cells (see Supporting Information Figure S6.4 for gating strategy). As the Spherotech beads are fluorescent, phagocytosis of the beads will cause an increase in fluorescent intensity of the cells (Figure 6.4, left panel). A higher fluorescence intensity indicates that more beads have been internalized. RAW cells only show one peak for 'empty' cells, whereas the cells incubated with NM-S beads, showed, next to empty cells, also peaks with a clear increase in fluorescence. The results show that NM-S beads 
were phagocytosed by the RAW cells, probably via complement or scavenger receptors that are directed against proteins that were non-specifically adsorbed to the NM-S beads. ${ }^{8}$ Different peaks can be distinguished for cells that have phagocytosed 1, 2, 3 and more than 3 beads per

NM-S

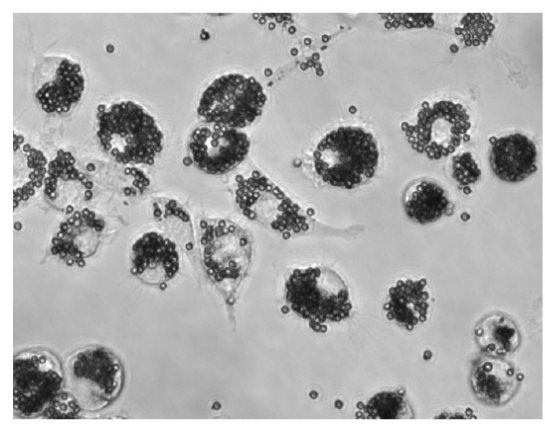

pSB-S

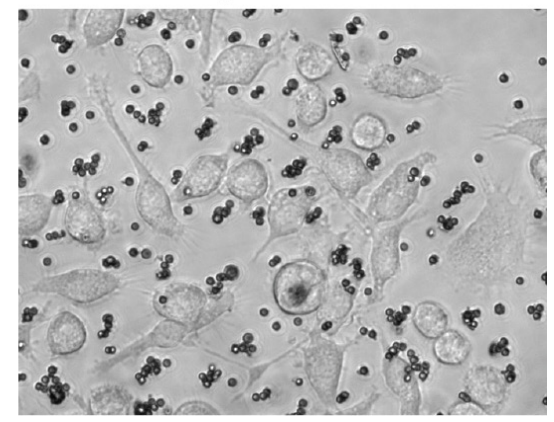

Figure 6.2 Optical images of RAW 264.7 cells after incubation with NM-S or pSB-S beads.

cell, as indicated in the graph. The relative amounts were quantified by gating and presented as bar plots (Figure 6.4, middle column). Of the cells incubated with NM-S beads, only 12\% of the cell population had not taken up any beads, while the rest of the cells did contain phagocytosed beads. The majority of cells (57\%) had internalized even more than 3 beads.

In strong contrast, incubation with pSB-S beads resulted in $88 \%$ empty cells, $7 \%$ of cells with 1 bead, $3 \%$ of cells with 2 beads and a negligible amount of cells with more than 2 beads per cell. This illustrates the ability of ZPB coatings to suppress phagocytosis by macrophages and confirms the initial results as obtained by light microscopy. In addition to the fluorescence

\section{RAW cells + NM-S beads}

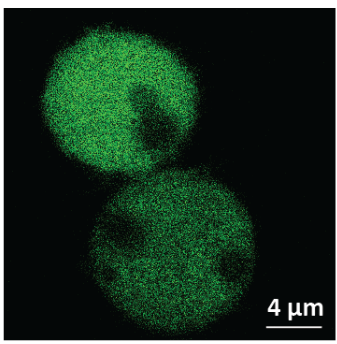

CFSE

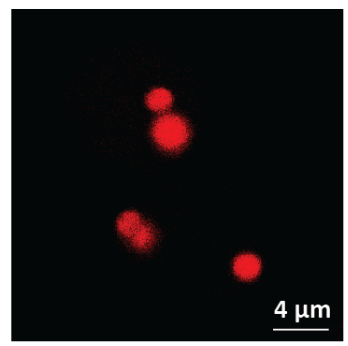

Beads

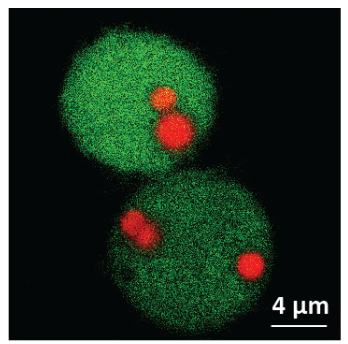

Overlay

Figure 6.3 Confocal microscopy image of CFSE-stained RAW 264.7 cells incubated with fluorescent NM-S beads. 
data, the cells with phagocytosed beads were also analyzed by evaluating their side (SSC) and forward (FSC) scattering. SSC is a measure of granularity, the degree of sub-cellular structures within a cell, whereas FSC is a measure of size. RAW cells without beads showed a relatively uniform population with low granularity. However, cells that were exposed to NM-S showed a significant increase in SSC, which can be explained by the increase in granularity due to internalized beads. In contrast, the pSB-S beads showed a similar population as the RAW cells only. The gates that were set to quantify the amount of beads per cell (as indicated in the left panel of Figure 6.4) were also used to color-code the cells presented in the right panel of Figure 6.4. The cells that internalized $>3$ beads (in blue) showed higher SSC as compared to cells with less beads, confirming that the SSC can also be used as a measure for the amount of beads that are taken up by the cells, albeit less accurately than by fluorescence.

\subsubsection{Selective phagocytosis of Dynabeads}

The pSB-S (Spherotech) beads showed a strong reduction in phagocytosis by RAW cells as compared to NM-S beads (Figure 6.2 and Figure 6.4). However, when the pSB-S beads were evaluated for their antifouling performance in protein solutions, these beads were not as antifouling as expected based on the phagocytosis assay (see Supporting Information Figure S6.5). We hypothesized that the Spherotech beads have a relatively low loading of amine groups at their surface, which is confirmed by the absence of a nitrogen peak in XPS wide scan spectra of the NM-S beads (see Supporting Information Figure S6.1C). The low surface concentration of amines on the beads would also result in a low initiator density, which is undesirable as a low initiator density has been shown to yield low-density coatings with inferior antifouling performance as compared to high-density brushes. ${ }^{27-28}$ In previous chapters we used Dynabeads, which are non-fluorescent but have high amine loading (see Supporting Information Figure S3.1) on which dense zwitterionic polymer brushes can be grown that show good antifouling capacity, ${ }^{29}$ and for which even better anti-phagocytosis character can be expected. Experiments on functionalized antifouling coatings were therefore continued using Dynabeads.

The ability to functionalize the antifouling particles with molecules for specific targeting is highly valuable for in vitro and in vivo applications. Here we show a proof of principle for this by using antifouling Dynabeads that were covalently functionalized with an antibody of the $\mathrm{IgG}_{2 \mathrm{a}}$ isotype. The $\mathrm{Fc}$ region of this antibody isotype can bind to $\mathrm{F}_{\mathrm{C}}$ receptors on RAW cells and thereby target the beads to these macrophages. To create zwitterionic polymer brush-coated Dynabeads that are also functionalizable, the beads were coated by the copolymerization of a standard SB monomer and a zwitterionic azide-functionalized monomer (azido-SB, see Supporting Information Scheme S6.1). We used 8\% of azido-SB monomer, yielding pSB-CO(azide) ${ }_{8 \%}$-D beads (see Figure 6.1). The incorporation of azide functionalities allows for the post-polymerization modification of the coatings with biomolecules via click chemistry. We first immobilized a TA99 IgG $_{2 a}$ antibody onto the beads to yield pSB-CO-(TA99) ${ }_{8 \%}$-D beads, via the click reaction of functionalized TA99 antibodies to PSB-co-(azide) ${ }_{8 \%}$-D beads, as described in detail in chapter 5 . The TA99 antibody recognizes tyrosinase-related protein 1 (TYRP1), which is a glycoprotein found in melanocytes. Within this study, TA99-functionalized beads were used 


\section{Spherotech beads phagocytosis by RAW cells}
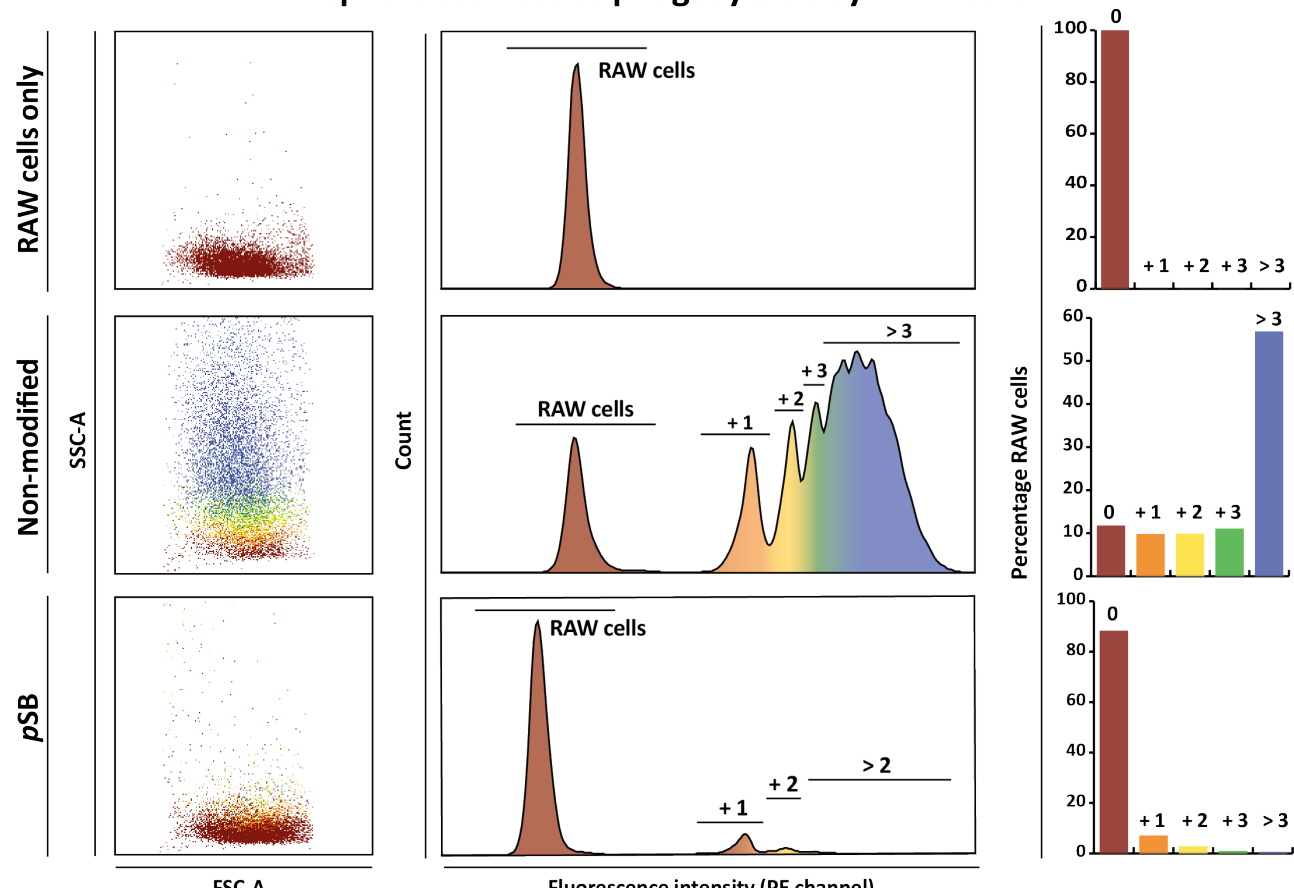

Figure 6.4 Flow cytometry analysis of live single cells: RAW cells only, RAW cells incubated with NM-S, and RAW cells incubated with pSB-S beads. The data are presented as histograms of fluorescence measured in the PE channel (left); data from histograms have been quantified and displayed as bar plots (middle), and SSC-A against FSC-A (right). Numbers indicate the number of beads per cell. Corresponding colors in histograms, bar plots and SSC/FSC plots indicate cell populations with similar numbers of phagocytosed beads.

as a positive control for FcyR-mediated phagocytosis. Non-modified (NM-D) and antifouling Dynabeads, with and without TA99, were used for a phagocytosis assay and analyzed by light microscopy (see Figure 6.5A-D) and flow cytometry. The results show that NM-D beads were phagocytosed by the RAW cells, whereas the phagocytosis of pSB-Co-(azide) ${ }_{8 \%}$-D beads was clearly prevented. The immobilization of TA99 on the antifouling beads (pSB-CO-(TA99) ${ }_{8 \%}-\mathrm{D}$ ) induced clear targeted phagocytosis of the beads by the macrophages. Phagocytosis of the pSBCO-(TA99) ${ }_{8 \%}$-D beads likely occurred via FC gamma receptor-mediated phagocytosis, ${ }^{30}$ which is phagocytosis induced by the binding of the Fc receptors on phagocytic cells to the Fc region on the antibodies.

To further assess the influence of immobilized proteins on the outside of the beads, pSB-co(azide) ${ }_{8 \%}$ beads were functionalized with ovalbumin (OVA) as model protein. To immobilize 
OVA onto the pSB-CO-(azide) ${ }_{8 \%}$ beads, OVA was first functionalized with a BCN-NHS linker; we will refer to the product as BCN-OVA (see Supporting Information Scheme S6.1). The NHS group of this linker reacts with the free amines of the OVA protein, while the BCN moiety allows for subsequent attachment to the azide-presenting beads via the Strain-Promoted Azide-Alkyne Cycloaddition (SPAAC) reaction. ${ }^{21,31}$ To evaluate whether the BCN-NHS linker was successfully attached to OVA, BCN-OVA was incubated with Azide-Lissamine (a fluorescent dye), as this should then yield an efficient SPAAC reaction, and analyzed by SDS-PAGE (see Figure 6.6A). The Coomassie stained gels showed clear OVA bands for untreated OVA, BCNOVA and Liss-OVA at the expected size of $43 \mathrm{kDa}$. The fluorescence image showed only a clear band after the reaction with Azide-Lissamine, confirming the successful labeling of BCN-OVA.
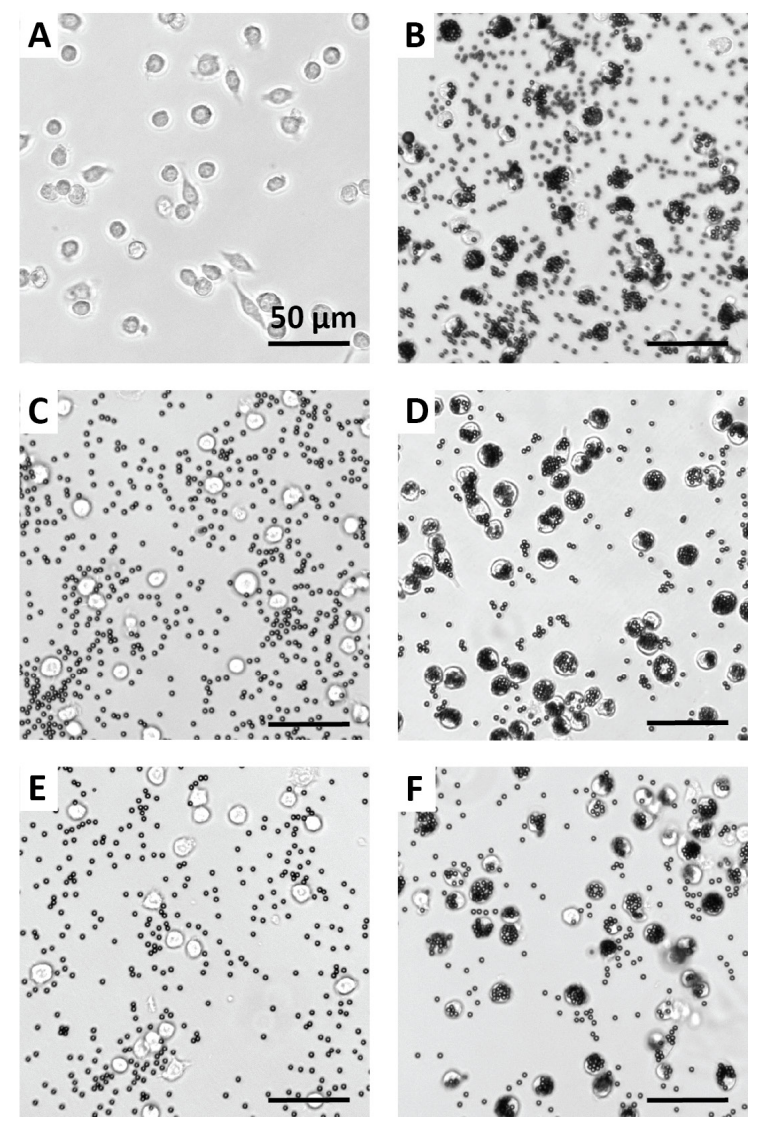

Figure 6.5 Phagocytosis assay analyzed by light microscopy using RAW 264.7 cells that were incubated with (A) no beads, (B) NM-D, (C) pSB-co-(azide) $)_{8 \%}-\mathrm{D}$, (D) pSB-co-(TA99) ${ }_{8 \%}-\mathrm{D}$, (E) pSB-co-(OVA) ${ }_{8 \%}-\mathrm{D}$, (F) ) pSB-co-(OVA $)_{8 \%}-\mathrm{D}$ labeled with anti-OVA $\operatorname{IgG}_{2 \mathrm{a}}$ antibody. In all panels the scale bar corresponds to $50 \mu \mathrm{m}$. 
BCN-OVA was then incubated with pSB-CO-(azide) ${ }_{8 \%}$ beads to yield the pSB-co-(OVA) ${ }_{8 \%}$ beads (see Figure 6.1). The attachment of OVA onto those beads was evaluated by incubating the beads with an anti-OVA antibody, and subsequent staining with a secondary anti-mouse-PE antibody. The evident shift in PE fluorescence confirmed the successful attachment of OVA on the beads (Figure 6.6B).

The prepared $\mathrm{pSB}-\mathrm{CO}-(\mathrm{OVA})_{8 \%}$ beads were subsequently subjected to the phagocytosis assay. Figure 6.5E shows that $\mathrm{pSB}-\mathrm{CO}-(\mathrm{OVA})_{8 \%}$ beads were not internalized, from which it can be concluded that the attachment of proteins such as OVA, does not directly induce phagocytosis by the macrophages. By binding an anti-OVA $\left(\operatorname{IgG}_{2 \mathrm{a}}\right.$ ) antibody (Figure 6.5F) to these pSBco-(OVA $)_{8 \%}$ beads, the macrophages were targeted to the beads and were thereby efficiently phagocytosed. This shows that the presence of the $\operatorname{IgG}_{2 \mathrm{a}}$ antibody induced the targeted phagocytosis (as with the TA99 antibody), and not the presence of an immobilized protein itself. The results from Figure 6.5 show therefore that, depending on the design of the bead, phagocytosis can either be induced or prevented.

The RAW cells were also analyzed by flow cytometry, see Figure 6.7. The data shown above (using Spherotech beads, Figure 6.4) demonstrated that the SSC of cells can be used to assess the degree of phagocytosis. The SSC was therefore also used to quantify the phagocytosis of the non-fluorescent Dynabeads. Only the macrophages that were incubated with either the NM-D or the antifouling beads linked to $\operatorname{IgG}_{2 a}$ showed an increased SSC and thus increased phagocytosis. The pSB-CO-(azide) ${ }_{8 \%}$ and pSB-CO-(OVA) ${ }_{8 \%}$ beads did not show significant changes in SSC as compared to RAW cells only, confirming the microscopic analysis that the RAW macrophages did basically not phagocytose these highly antifouling beads. The functionalized pSB-CO-(OVA $)_{8 \%}$ beads do therefore display a nearly ideal stealth character. Besides that, cells incubated with antifouling Dynabeads (pSB-co-(azide) ${ }_{8 \%}$ and $\mathrm{pSB}-\mathrm{CO}-(\mathrm{OVA})_{8 \%}$ ) showed no

A

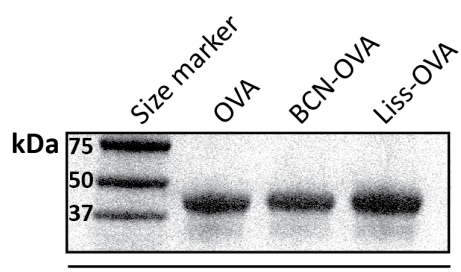

Coomassie staining

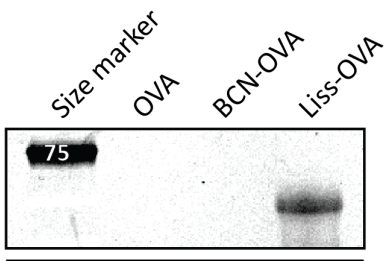

Fluorescence
B

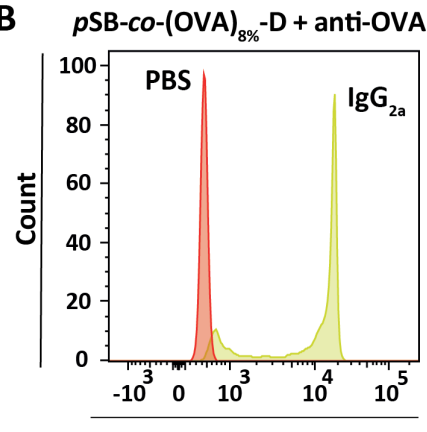

PE fluorescence intensity

Figure 6.6 Evaluation of BCN-NHS coupling to OVA by SDS-PAGE. Untreated OVA, OVA labeled with $\mathrm{BCN}-\mathrm{NHS}$ (BCN-OVA), and BCN-OVA reacted with Azide-Lissamine (Liss-OVA) were visualized by Coomassie staining and fluorescence. (B) Flow cytometry data of pSB-co-(OVA) ${ }_{8 \%}$ beads incubated with anti-OVA $\left(\operatorname{IgG}_{2 \mathrm{a}}\right)$ stained with anti-Mouse-PE antibody. 
differences compared to RAW cells that were not brought into contact with the beads. This suggests that antifouling Dynabeads, with their denser zwitterionic polymer brush layers, are better in preventing phagocytosis than the Spherotech beads, which showed that about $10 \%$ of cells phagocytosed one or two pSB-S beads. This is in line with the aforementioned better antifouling performance of the Dynabeads as compared to the Spherotech beads, and confirms the need of high performance antifouling layers to fully prevent phagocytosis.

Taken together, the results illustrate the potential of zwitterionic polymer coatings for in vitro and in vivo use. The immobilization of a protein itself (here OVA) does not affect the stealth characteristics of the coated beads, as is demonstrated by their ability to almost completely resist phagocytosis. The fate of the antifouling bead can therefore be determined by the introduction of a targeting molecule, as we have shown for the $\mathrm{IgG}_{2 \mathrm{a}}$ antibodies that target $\mathrm{Fc}$ gamma receptors on macrophages. Such particles with stealth character can be utilized in various applications, such as the direct targeting of antigen-presenting cells (APCs) such as macrophages (as we have shown), which can be of use to enhance antigen presentation to boost vaccination efficiency. ${ }^{32}$ Especially microbeads of 2-3 $\mu \mathrm{m}$ size (as our beads of $2.8 \mu \mathrm{m}$ ) have been shown to be adequate candidates for particle-based vaccine development. ${ }^{33-34}$ Secondly, zwitterionic polymer brush-coated particles can be used as drug delivery carriers to enhance transport of the drug to the targeted site (e.g. tumor). Thirdly, functionalized antifouling particles can be used for the isolation of cells or proteins in the presence of APCs, without being cleared from the solution. It should be noted that when antibodies are used to target stealth beads to specific cells, the antibodies should not contain the Fc regions that can bind to Fc receptors in order to prevent unwanted phagocytosis. For this purpose, only the antigen-binding part (Fab and $\mathrm{F}(\mathrm{ab})_{2}$ fragments) of antibodies, llama antibodies or nanobodies should be used. In all these examples, a reduction of the non-specific protein adsorption onto the functionalized particles and corresponding ability to resist phagocytosis, would greatly improve the performance of those particles.

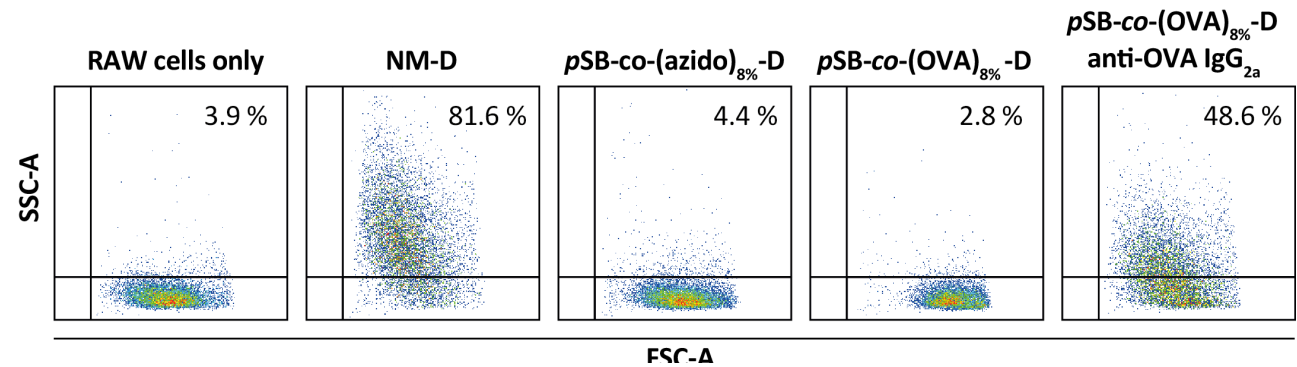

Figure 6.7 Flow cytometry SSC plots of RAW cells incubated in RPMI medium, or incubated with NMD, pSB-co-(azide) $)_{8 \%}-\mathrm{D}, \mathrm{pSB}-\mathrm{CO}-(\mathrm{OVA})_{8 \%}-\mathrm{D}$, or pSB-cO-(OVA $)_{8 \%}-\mathrm{D}$ beads labeled with anti-OVA IgG ${ }_{2 \mathrm{a}}$. 


\subsection{Conclusion}

In this study it was shown that microbeads coated with antifouling zwitterionic polymer brushes, grown via surface-initiated polymerization, can effectively prevent phagocytosis. Non-modified beads were efficiently internalized by murine macrophages (RAW 264.7 cells), whereas the zwitterionic polymer brush-coated beads were only scarcely taken up , and thereby remained nearly undetectable for the macrophages. Also immobilization of ovalbumin on the antifouling beads did not increase their phagocytosis by macrophages, meaning that such OVAfunctionalized zwitterionic polymer brush-coated beads keep their stealth characteristics. In contrast, the immobilization of $\operatorname{IgG}_{2 \mathrm{a}}$ antibodies onto OVA-functionalized beads enabled the specific targeting of the beads to the Fc gamma receptors on the macrophages, inducing active internalization of the antifouling beads. The results show the high potential of functionalized zwitterionic polymer coatings for targeted in vivo delivery with numerous biomedical applications, like theranostics and vaccine development.

\subsection{Acknowledgments}

The authors thank Ben Meijer and Jan Willem Borst for technical assistance regarding flow cytometry measurements and confocal microscopy, respectively. Jorick Bruins is thanked for providing the Azide-Lissamine and Jorge Cuenca Escalona for assistance in the lab. This work was supported by NanoNextNL (program 3E), a micro and nanotechnology consortium of the government of The Netherlands and 130 partners.

\subsection{References}

1. Owens, D. E.; Peppas, N. A. Opsonization, Biodistribution, and Pharmacokinetics of Polymeric Nanoparticles. Int.J. Pharm. 2006, 307, 93-102.

2. Ahmed, N.; Fessi, H.; Elaissari, A. Theranostic Applications of Nanoparticles in Cancer. Drug Discovery Today 2012, 17, 928934.

3. Zhang, K.-L.; Zhou, J.; Zhou, H.;Wu,Y.; Liu, R.;Wang, L.-L.; Lin, W.-W.; Huang, G.; Yang, H.-H. Bioinspired “Active” Stealth Magneto-Nanomicelles for Theranostics Combining Efficient MRI and Enhanced Drug Delivery. ACS Appl. Mater. Interfaces 2017, 9, 30502-30509.

4. Zhu, L.; Zhou, Z.Y.; Mao, H.; Yang, L. L. Magnetic Nanoparticles for Precision Oncology:Theranostic Magnetic Iron Oxide Nanoparticles for Image-Guided and Targeted Cancer Therapy. Nanomedicine 2017, 12, 73-87.

5. Gref, R.; Minamitake, Y.; Peracchia, M. T.; Trubetskoy, V.; Torchilin, V.; Langer, R. Biodegradable Long-Circulating Polymeric Nanospheres. Science 1994, 263, 1600-1603.

6. Salvati, A.; Pitek, A. S.; Monopoli, M. P.; Prapainop, K.; Bombelli, F. B.; Hristov, D. R.; Kelly, P. M.; Aberg, C.; Mahon, E.; Dawson, K. A. Transferrin-Functionalized Nanoparticles Lose Their Targeting Capabilities When a Biomolecule Corona Adsorbs on the Surface. Nat. Nanotechnol. 2013, 8, 137-143.

7. Pareek, V.; Bhargava, A.; Bhanot, V.; Gupta, R.; Jain, N.; Panwar, J. Formation and Characterization of Protein Corona around Nanoparticles: A Review. J. Nanosci. Nanotechnol. 2018, 18, 6653-6670. 
8. Ishida, T.; Harashima, H.; Kiwada, H. Interactions of Liposomes with Cells in Vitro and in Vivo: Opsonins and Receptors. Curr. Drug Metab. 2001, 2, 397-409.

9. Garcia, K. P.; Zarschler, K.; Barbaro, L.; Barreto, J. A.; O’Malley, W.; Spiccia, L.; Stephan, H.; Graham, B. Zwitterionic-Coated "Stealth" Nanoparticles for Biomedical Applications: Recent Advances in Countering Biomolecular Corona Formation and Uptake by the Mononuclear Phagocyte System. Small 2014, 10, 2516-2529.

10. Karakoti, A. S.; Das, S.; Thevuthasan, S.; Seal, S. PEGylated Inorganic Nanoparticles. Angew. Chem. Int. Ed. 2011, 50, $1980-1994$.

11. Otsuka, H.; Nagasaki, Y.; Kataoka, K. PEGylated Nanoparticles for Biological and Pharmaceutical Applications. Adv. Drug Delivery Rev. 2003, 55, 403-419.

12. Ladd, J.; Zhang, Z.; Chen, S.; Hower, J. C.; Jiang, S. Zwitterionic Polymers Exhibiting High Resistance to Nonspecific Protein Adsorption from Human Serum and Plasma. Biomacromolecules 2008, 9, 1357-1361.

13. Ostuni, E.; Chapman, R. G.; Holmlin, R. E.;Takayama, S.; Whitesides, G. M. A Survey of Structure-Property Relationships of Surfaces That Resist the Adsorption of Protein. Langmuir 2001, 17, 5605-5620.

14. Li, L. Y.; Chen, S. F.; Jiang, S.Y. Protein Interactions with Oligo(Ethylene Glycol) (OEG) Self-Assembled Monolayers: Oeg Stability, Surface Packing Density and Protein Adsorption. J. Biomater. Sci., Polym. Ed. 2007, 18, 1415-1427.

15. Kawai, F. Microbial Degradation of Polyethers. Appl. Microbiol. Biotechnol. 2002, 58, 30-38.

16. Armstrong, J. K.; Hempel, G.; Koling, S.; Chan, L. S.; Fisher, T.; Meiselman, H. J.; Garratty, G. Antibody against Poly(Ethylene Glycol) Adversely Affects PEG-Asparaginase Therapy in Acute Lymphoblastic Leukemia Patients. Cancer 2007, 110, 103111.

17. Schellekens, H.; Hennink, W. E.; Brinks, V.The Immunogenicity of Polyethylene Glycol: Facts and Fiction. Pharm. Res. 2013, 30, 1729-1734.

18. Jiang, S. Y.; Cao, Z. Q. Ultralow-Fouling, Functionalizable, and Hydrolyzable Zwitterionic Materials and Their Derivatives for Biological Applications. Adv. Mater. 2010, 22, 920-932.

19. Schlenoff, J. B. Zwitteration: Coating Surfaces with Zwitterionic Functionality to Reduce Nonspecific Adsorption. Langmuir 2014, 30, 9625-9636.

20. Wei, Q.; Becherer, T.; Angioletti-Uberti, S.; Dzubiella, J.;Wischke, C.; Neffe, A. T.; Lendlein, A.; Ballauff, M.; Haag, R. Protein Interactions with Polymer Coatings and Biomaterials. Angew. Chem. Int. Ed. 2014, 53, 8004-8031.

21. Lange, S. C.; van Andel, E.; Smulders, M. M. J.; Zuilhof, H. Efficient and Tunable Three-Dimensional Functionalization of Fully Zwitterionic Antifouling Surface Coatings. Langmuir 2016, 32, 10199-10205.

22. Blaszykowski, C.; Sheikh, S.; Thompson, M. A Survey of State-of-the-Art Surface Chemistries to Minimize Fouling from Human and Animal Biofluids. Biomater. Sci. 2015, 3, 1335-1370.

23. Zhang, L.; Sun, Y. Poly(Carboxybetaine Methacrylate)-Grafted Silica Nanoparticle: A Novel Carrier for Enzyme Immobilization. Biochemical Engineering Journal 2018, 132, 122-129.

24. Hu, R.; Li, G.; Jiang,Y.; Zhang, Y.; Zou, J.-J.;Wang, L.; Zhang, X. Silver-Zwitterion Organic-Inorganic Nanocomposite with Antimicrobial and Antiadhesive Capabilities. Langmuir 2013, 29, 3773-3779.

25. Yang, W.; Liu, S.; Bai, T.; Keefe, A. J.; Zhang, L.; Ella-Menye, J.-R.; Li, Y.; Jiang, S. Poly(Carboxybetaine) Nanomaterials Enable Long Circulation and Prevent Polymer-Specific Antibody Production. Nano Today 2014, 9, 10-16.

26. Piazzon, M. C.; Savelkoul, H. S.; Pietretti, D.; Wiegertjes, G. F.; Forlenza, M. Carp IL10 Has Anti-Inflammatory Activities on Phagocytes, Promotes Proliferation of Memory T Cells, and Regulates B Cell Differentiation and Antibody Secretion. J. Immunol. 2015, 194, 187-99.

27. Jones, D. M.; Brown, A. A.; Huck, W.T. S. Surface-Initiated Polymerizations in Aqueous Media: Effect of Initiator Density. Langmuir 2002, 18, 1265-1269.

28. Dalsin, J. L.; Messersmith, P. B. Bioinspired Antifouling Polymers. Mater.Today 2005, 8, 38-46.

29. van Andel, E.; de Bus, I.; Tijhaar, E. J.; Smulders, M. M. J.; Savelkoul, H. F. J.; Zuilhof, H. Highly Specific Binding on Antifouling Zwitterionic Polymer-Coated Microbeads as Measured by Flow Cytometry. ACS Appl. Mater. Interfaces 2017, 9 , 
38211-38221.

30. Aderem, A.; Underhill, D. M. Mechanisms of Phagocytosis in Macrophages. Annu. Rev. Immunol. 1999, 17, 593-623.

31. Escorihuela, J.; Marcelis, A. T. M.; Zuilhof, H. Metal-Free Click Chemistry Reactions on Surfaces. Adv. Mater. Interfaces 2015, 2 , 1500135 .

32. Mottram, P. L.; Leong, D.; Crimeen-Irwin, B.; Gloster, S.; Xiang, S. D.; Meanger, J.; Ghildyal, R.; Vardaxis, N.; Plebanski, M. Type 1 and 2 Immunity Following Vaccination Is Influenced by Nanoparticle Size: Formulation of a Model Vaccine for Respiratory Syncytial Virus. Mol. Pharm. 2007, 4, 73-84.

33. Champion, J.A.; Walker, A.; Mitragotri, S. Role of Particle Size in Phagocytosis of Polymeric Microspheres. Pharm. Res. 2008, 25, 1815-1821.

34. Xiang, S. D.; Scholzen, A.; Minigo, G.; David, C.; Apostolopoulos, V.; Mottram, P. L.; Plebanski, M. Pathogen Recognition and Development of Particulate Vaccines: Does Size Matter? Methods 2006, 40, 1-9. 


\subsection{Supporting Information}

Fluorescent Spherotech beads (S)

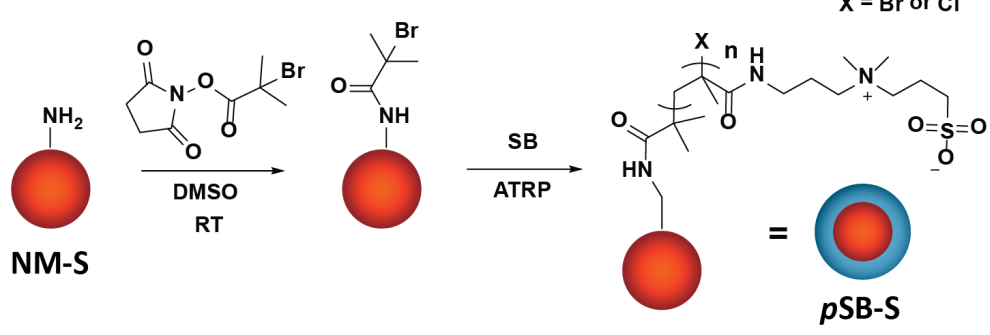

Dynabeads (D)
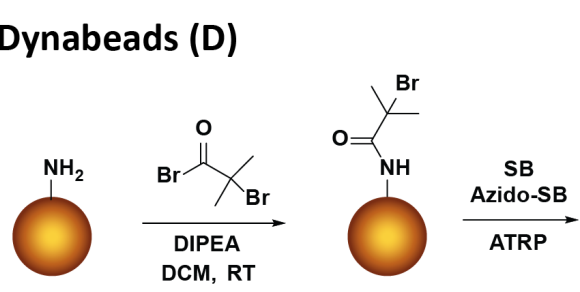

NM-D

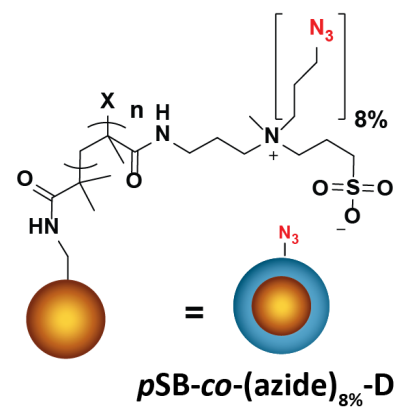

OVA functionalization with BCN-NHS
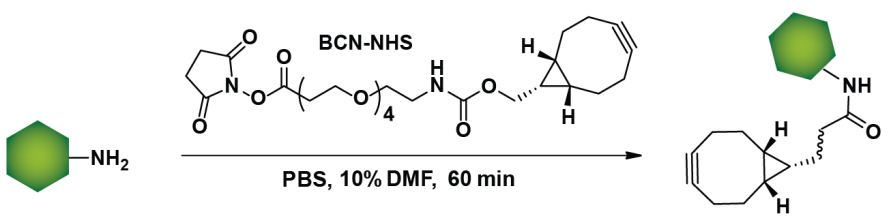

BCN-OVA

BCN-OVA immobilization on bead
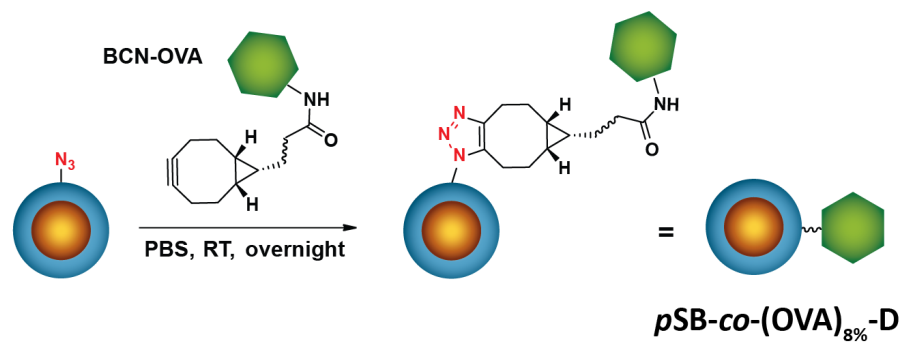

Scheme S6.1 Schematic representation of modification on beads. 


\subsubsection{XPS spectra}
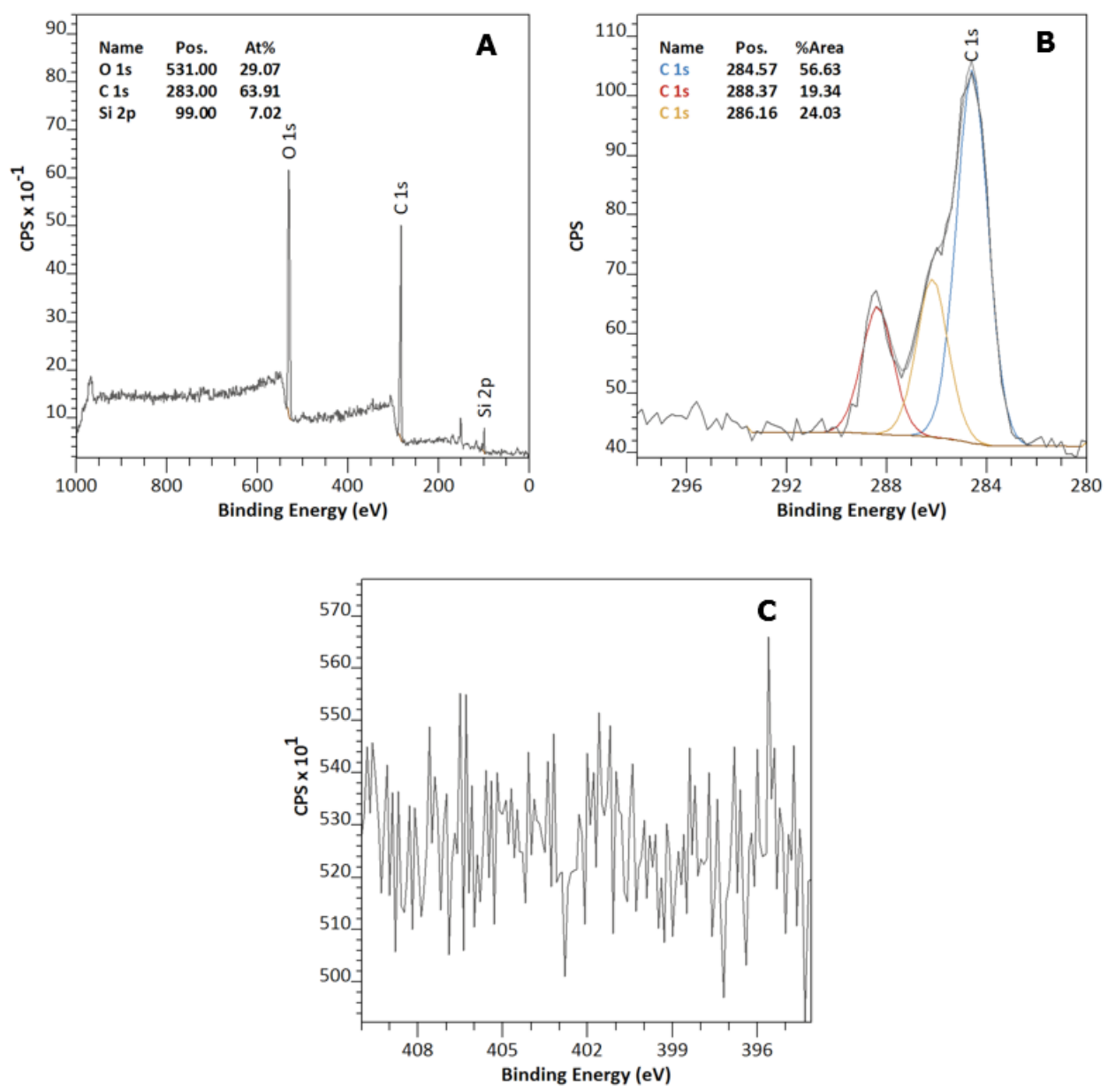

Figure S6.1 XPS spectra of non-modified Spherotech beads (NM-S). (A) wide scan spectrum, (B) C 1s narrow scan spectrum, (C) N 1s narrow scan spectrum. Please note that the beads were dropcasted on a $\mathrm{Si}(111)$ surface to allow for XPS analysis (hence the Si peaks in the wide scan spectrum). 

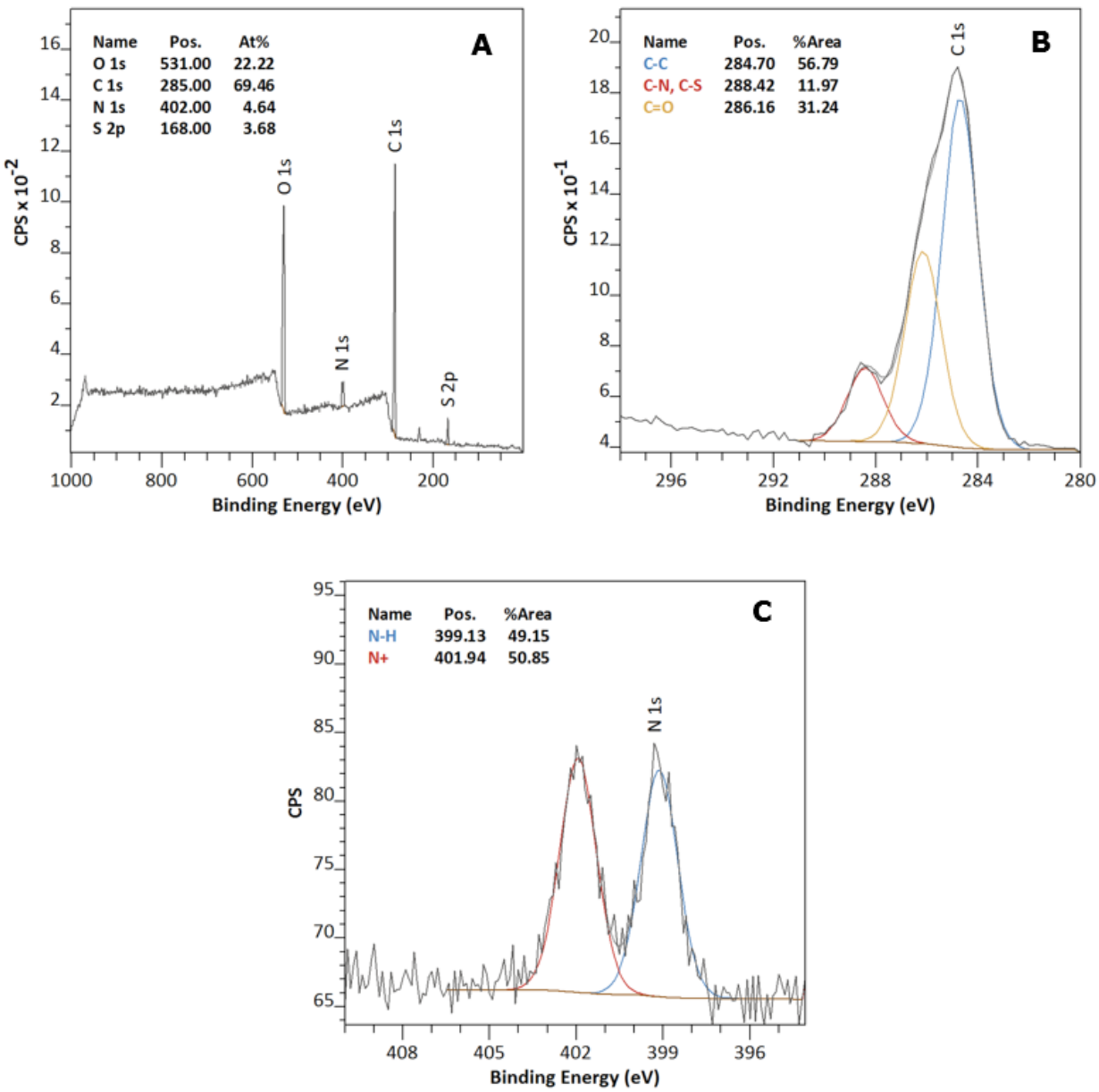

Figure S6.2 XPS spectra of Spherotech beads coated with SB polymer brushes (pSB-S) grown via SIATRP. (A) wide scan spectrum, (B) C 1s narrow scan spectrum, (C) N 1s narrow scan spectrum. 


\subsubsection{Additional confocal microscopy images}

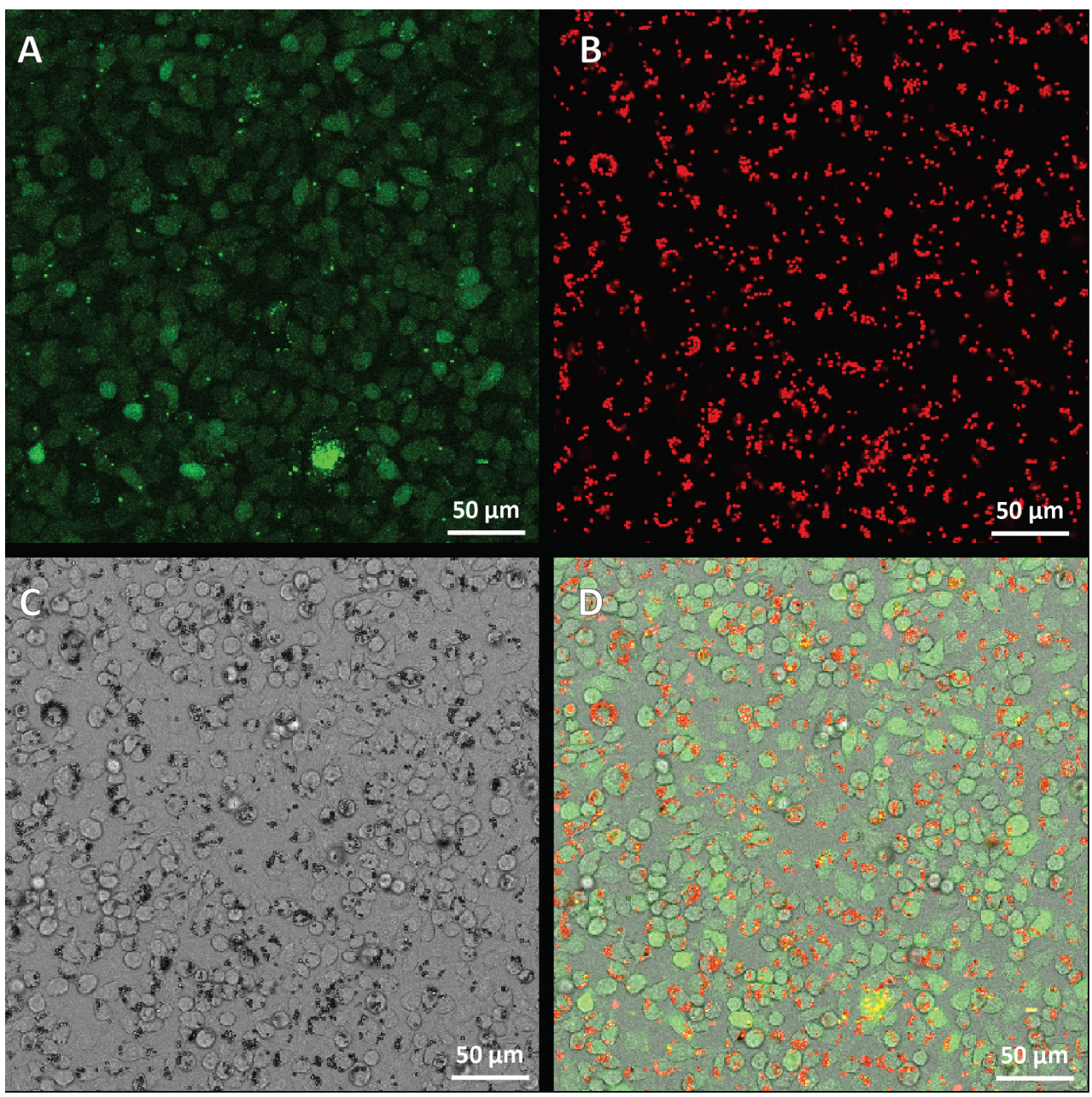

Figure S6.3 Confocal images of RAW 264.7 cells after incubation with NM-S beads. (A) panel showing CFSE-stained cells in green, (B) showing beads in red, (C) transmission image, and (D) overlay image of image A-C. 


\subsubsection{Additional flow cytometry data}

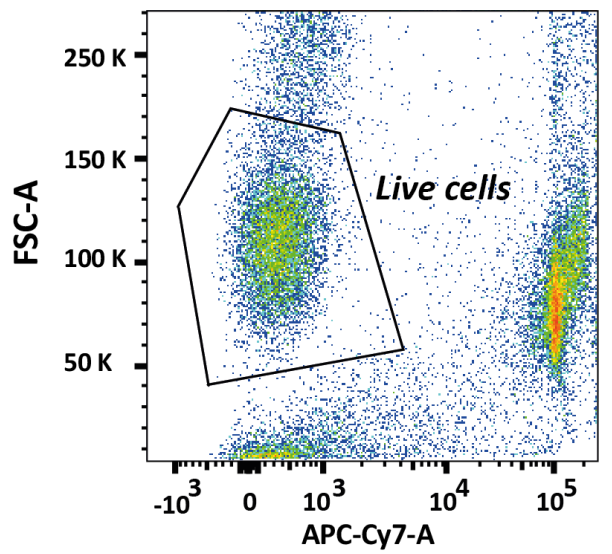

Figure S6.4 Example of gating on single live cells using DRAQ7 as dead/live marker (DRAQ7 stains dead cells). The events with high fluorescence intensity therefore represent the dead cells. The cells within in the gate are considered single live cells and were used for further analysis. Both Spherotech beads as well as Dynabeads were gated this way.

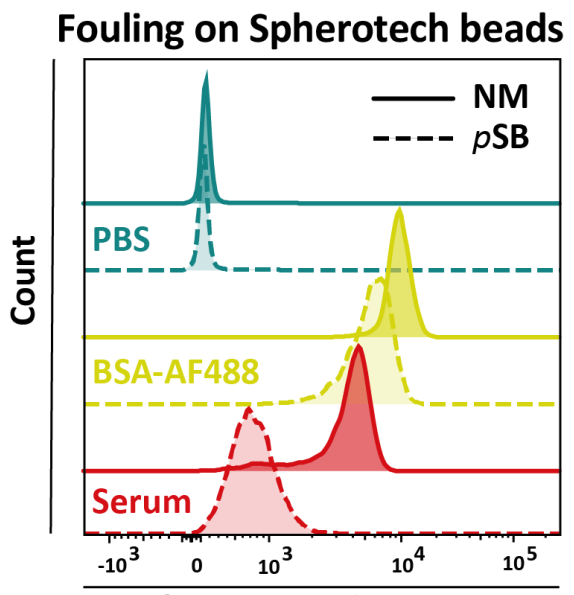

Fluorescence intensity

Figure S6.5 Fouling on Spherotech beads as measured by flow cytometry. Non-modified beads (NM, solid lines) and pSB-coated beads (dashed lines) were incubated in either PBS, BSA-AF488 $(0.5 \mathrm{mg} / \mathrm{mL}$ in PBS) or by $10 \%$ biotinylated bovine serum followed by Streptavidin-FITC. A higher fluorescence intensity indicates that more proteins have adsorbed on the beads, i.e. a higher degree of fouling. 


\subsubsection{Preparation of antifouling Spherotech beads}

To be able to study the phagocytic uptake of beads by confocal microscopy and flow cytometry, fluorescent amine-terminated micron-sized beads were selected as starting point: SPHERO ${ }^{\text {TM }}$ Amino Fluorescent Pink Magnetic Particles (Spherotech beads, S). To obtain antifouling polymer-coated beads via Surface-Initiated Atom-Transfer Radical Polymerization (SI-ATRP) as we previously reported (chapter 3-5), ${ }^{\text {S1 }}$ first an ATRP-initiator needed to be coupled to the beads. To this end, the amine-terminated Spherotech beads (NM-S, see Scheme S6.1) were reacted with $\alpha$-bromoisobutanoic acid N-hydroxysuccinimide ester in dry DMSO. This was followed by the surface-initiated polymerization of a standard sulfobetaine monomer (SB) to yield the antifouling polymer brush-coated Spherotech beads (pSB-S). The growth of the polymer brushes was evaluated by X-ray Photoelectron Spectroscopy (XPS), see Supporting Information Figure S6.2. The XPS wide scan spectrum, which shows the elemental composition of the material analyzed, showed the appearance of two sulfur peaks at $168 \mathrm{eV}$ (S 2p) and 229 $\mathrm{eV}$ (S 2s), which both correspond to the sulfur atom in the sulfonate group of the sulfobetaine monomer. When looking in more detail to the analyzed elements, the nitrogen narrow scan showed the 1 : 1 ratio of the positively charged ammonium $(399.1 \mathrm{eV})$ and $(401.9 \mathrm{eV})$ peaks, that is characteristic for the amide-linked sulfobetaine polymer brushes. ${ }^{\text {s1-s2 }}$ In addition, the XPS C 1s spectrum could be deconvoluted in three peaks: the $\mathrm{C}-\mathrm{C}$ peak at $284.7 \mathrm{eV}$, the peak corresponding to the carbon atoms next to a heteroatom $(\mathrm{C}-\mathrm{N}$, and $\mathrm{C}-\mathrm{S})$ at $288.4 \mathrm{eV}$ and the carbonyl peak at $286.2 \mathrm{eV}$. Taken together, the XPS results confirm the successful zwitterionic brush growth on Spherotech beads.

\subsubsection{References}

S1. van Andel, E.; de Bus, I.; Tijhaar, E. J.; Smulders, M. M. J.; Savelkoul, H. F. J.; Zuilhof, H. Highly Specific Binding on Antifouling Zwitterionic Polymer-Coated Microbeads as Measured by Flow Cytometry. ACS Appl. Mater. Interfaces 2017, 9 , 38211-38221.

S2. Lange, S. C.; van Andel, E.; Smulders, M. M. J.; Zuilhof, H. Efficient and Tunable Three-Dimensional Functionalization of Fully Zwitterionic Antifouling Surface Coatings. Langmuir 2016, 32, 10199-10205. 


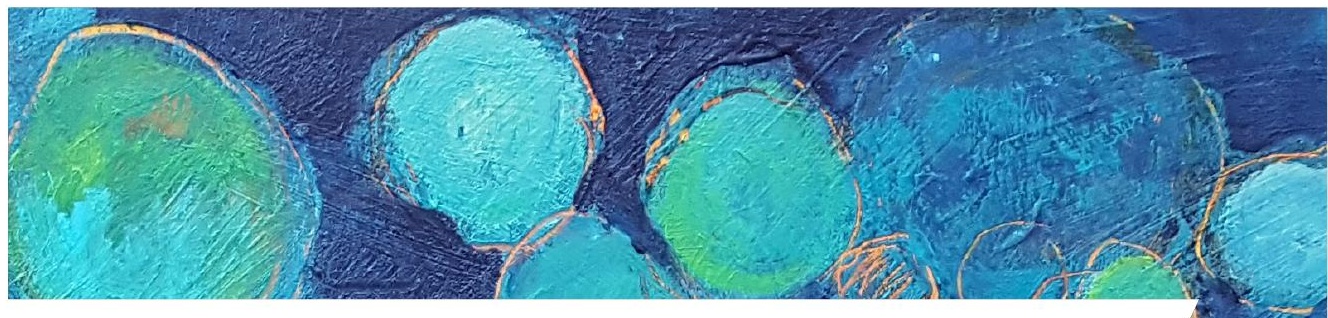

\section{Chapter.}




\section{General Discussion}


It is evident from the large number of research papers that have been published on the prevention of fouling in biomedical applications, that fouling is a considerable problem that hampers the performance of, among others, biosensors, drug delivery carriers and indwelling devices. In previous chapters new tools and insights were presented to expand the usability of zwitterionic polymer brushes in biomedical applications. In this chapter, the most important findings of each chapter are discussed and recommendations are given for future research. 


\subsection{Chapter 2: Efficient and Tunable Three- Dimensional Functionalization of Fully Zwitterionic Antifouling Surface Coatings}

As outlined in chapters 1 and 2, romantic surfaces ${ }^{1}$ are highly desired for diagnostic purposes. Surfaces that only bind the target biomolecule and repel all other unwanted material can greatly improve the signal-to-noise ratio and thereby improve the sensitivity and selectivity of the diagnostic measurement. A protein-repellent surface alone will not suffice; recognition units are required that need to be immobilized on the antifouling coating. This poses an intrinsic challenge: incorporating functional units - which are not antifouling by themselves - are prone to deteriorate the protein-repellent capacity of the antifouling coating. For this reason, we aimed to develop a method that introduces functionality to an antifouling brush, while maintaining the overall highly zwitterionic nature of the brush. A monomer that combines both zwitterionic character and functionality within a single molecule was therefore a promising approach. Chapter 2 described the synthesis of a novel sulfobetaine-based monomer that is equipment with an azide functionality. The copolymerization of this azido-SB monomer with a standard sulfobetaine resulted in 3D-functionalizable antifouling zwitterionic polymer brushes that were grown from silicon nitride surfaces. Subsequent functionalization of the brushes with biotin enabled the specific binding of avidin and prevented the non-specific binding of fibrinogen, thus demonstrating the desired romantic character of the modified surface.

\subsubsection{Surface chemistry}

Although the emphasis of chapter 2 is on the synthesis, polymerization and functionalization of the azido-SB monomer, we also developed a reproducible and scalable method to grow polymer brushes from stoichiometric silicon nitride $\left(\mathrm{Si}_{3} \mathrm{~N}_{4}\right)$ surfaces, which turned out to be non-trivial. The stability of the polymer is important for reliable sensing measurements and it has been shown that most degradation of polymer brushes occurs at the surface interphase..$^{2-4}$ It is therefore crucial to develop a stable attachment chemistry. We modified stoichiometric silicon nitride surfaces for future use in mirroring resonator or other optofluidics-based sensing devices. ${ }^{5-6}$ Alkyne/alkene chemistry on Si-based substrates has been shown to give stable and well-ordered layers, ${ }^{7-8}$ outperforming silane- or phosphonic acid-based surface chemistries. The modification of silicon-enriched silicon nitride using functionalized alkenes was reported before ${ }^{9-10}$ and can be performed analogously to alkyne/alkene chemistry on hydrogen-terminated silicon surfaces. ${ }^{11}$ Initially, we used the same modification procedures as reported for hydrogen-terminated silicon on our $\mathrm{Si}_{3} \mathrm{~N}_{4}$ surfaces, but this turned out to be non-satisfactory (both in terms of reproducibility as well as in amount of incorporated functionality). It has been reported that the surface groups of freshly etched $\mathrm{Si}_{3} \mathrm{~N}_{4}$ surfaces consist primarily of $\mathrm{Si}-\mathrm{F}$ (as also seen by the inorganic $\mathrm{F}$ that we observed in the XPS narrow scan; see chapter 2 Supporting Information Figures S18 and S19), Si-OH, Si-O-Si and Si$\mathrm{NH}_{2}$ groups, and does not contain significant amounts of $\mathrm{Si}-\mathrm{H}$ (as is the case for the siliconenriched silicon nitride surfaces). ${ }^{12-13}$ This explains why the usage of alkenes/alkynes under 
mild conditions on the $\mathrm{Si}_{3} \mathrm{~N}_{4}$ surfaces, primarily targeting $\mathrm{Si}-\mathrm{H}$ groups, was unsuccessful. To modify the $\mathrm{Si}_{3} \mathrm{~N}_{4}$ surfaces with alkenes, harsher conditions were needed to be able to react the alkenes with the nitrogen/oxygen-containing functional groups (we employed $150{ }^{\circ} \mathrm{C}$ overnight using the neat alkene under argon atmosphere). That the alkenes indeed reacted with the nitrogen/oxygen species was confirmed by the atom ratios obtained from XPS analysis. The carbon narrow scan (see chapter 2, Figure 2) showed an atomic ratio of $1.0: 1.1: 2.6: 7.4$ for the (from left to right) $\mathrm{CF}_{3}$, carbonyl, $\mathrm{C}-\mathrm{O} / \mathrm{C}-\mathrm{N}$ and $\mathrm{C}-\mathrm{C}$ peaks, which is in good agreement with the expected ratios of $1: 1: 2: 8$.

\subsubsection{Introducing functionality to zwitterionic polymer brushes}

Various methods have been developed to introduce functionality to antifouling coatings. ${ }^{14-20}$ As mentioned in previous chapters, the direct functionalization of CB-based brushes via EDC/NHS chemistry is probably the most widely used approach. ${ }^{21-22}$ This type of functionalization has yielded various examples of polymer brushes capable of binding target molecules with high specificity from complex protein mixtures. ${ }^{20,23}$ The main advantage of this approach is that standard CB monomers can be used that can be functionalized with readily available chemicals according to established protocols. ${ }^{22}$ However, upon functionalization of the carboxyl groups, the zwitterionic character of the polymer brushes is partially lost. ${ }^{21}$ This type of functionalization changes the key feature of antifouling zwitterionic coatings: the combination of highly polar moieties with an overall zero net charge. Besides that, the degree of functionalization is difficult to control and is highly dependent on the exact reaction conditions such as buffer composition, reagent concentration and type of coating. ${ }^{24-27}$ It has been demonstrated that even without the incorporation of recognition elements, fouling can increase on CB-based brushes upon EDC/NHS activation and subsequent deactivation of the CB carboxyl groups. ${ }^{21,28}$ It remains challenging to fully deactivate the unreacted activated carboxyl groups, which decreases the antifouling capacity. Besides these drawbacks, initial studies in our laboratory showed degradation of $\mathrm{CB}$ brushes upon storage (as also described in chapter 4). This underlines the need to develop a method in which functionality can be introduced to zwitterionic coatings without the use of carboxybetaines, as demonstrated in chapter 2.

Another approach to introduce functionality to polymer brushes is the copolymerization of functional non-zwitterionic monomers within a brush, ${ }^{14,30}$ which has the advantage that the number of functional groups can be tuned, and that the amount of available functional groups (in the top part of the polymer brush) is independent of brush thickness. Our approach using the zwitterionic azido-SB monomer has the same beneficial features, with the additional improvement of a fully zwitterionic polymer brush. Hu and Emrick $^{29}$ developed parallel to our work a method in which they equipped a choline phosphate (reverse from phosphocholine, see Scheme 7.1) monomer with an alkyne, which they further functionalized via coppermediated click chemistry. The polymers were grown in solution, and as a result the antifouling properties and specific binding capabilities were not investigated. Parallel to the synthesis of the azido-sulfobetaine we worked on a similar molecule (see Scheme 7.1): a phosphocholine with alkyne functionality. However, we encountered spontaneous polymerization ${ }^{31}$ and this 
made it very challenging to handle, purify and store the functionalized monomer. We therefore continued with the sulfobetaine. Considering the results obtained in chapter 4 , in which the phosphocholine clearly outperforms the sulfobetaines, it is still of interest to evaluate and compare both the binding capacity and the antifouling performance of the azido-SB (or of the azido-SB analog with carbon spacer length of two methylene groups between the opposite charges) with the alkyne-functionalized choline phosphates and phosphocholines. It would then also be interesting to evaluate whether the alkyne-functionalized CP shows similar selfpolymerization behavior as the $\mathrm{PC}$, and whether the functional zwitterionic monomers can indeed outperform CB brushes that are functionalized via EDC/NHS chemistry. This comparison could give insight to which degree and under what experimental conditions the extra synthetic effort to make the novel monomers is justified by their improved performance.

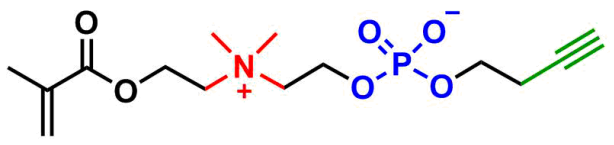

Alkyne-functionalized CP

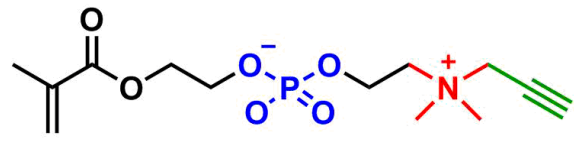

Alkyne-functionalized PC

Scheme 7.1 Chemical structures of alkyne-functionalized choline phosphate (CP) monomer as described by Hu et al.29 and alkyne-functionalized phosphocholine (PC) monomer. Alkynes are indicated in green, quaternary ammonium cations in red and phosphonate anions in blue.

A small functional group as the azide (or alkyne) was chosen as a reactive handle, as it is less likely to disrupt the brush conformation and/or influence the antifouling capacity. The incorporation of an azide moiety allows for the functionalization via the $\mathrm{CuAAC}^{32}$ (chapter 3) and SPAAC ${ }^{33-34}$ (chapter 2-6) click reactions. The copper-free SPAAC reaction is attractive as commercially available functional strained alkynes (e.g. BCN-biotin or BCN-NHS) can be used, which can simply be added to the azide-bearing coatings without the need of other reagents. The definition of a click reaction, as introduced by Sharpless and co-workers, states that a click reaction should be modular, give high yield, have a wide scope, produce minimal side products and should work under mild reactions. ${ }^{35}$ However, high yields are not always easily obtained, especially at surfaces, ${ }^{36-37}$ which has most likely to do with steric hindrance. It could therefore be interesting to introduce higher yielding click reactions as this might lower the amount of required functional groups that need to be incorporated in the polymer brushes, likely improving the antifouling performance. A click reaction that yields quantitative conversion is the sulfur fluoride exchange (SuFEx) reaction, which was introduced by Sharpless and co-workers. ${ }^{38}$ SuFEx has recently been made applicable for surface-based reactions. ${ }^{39-40}$ Even at the surface, SuFEx yields (near-)quantitative conversion. The surfacetethered sulfonyl fluoride can react with silyl ethers or primary amines (see Scheme 7.2). The reactivity of SuFEx towards amines makes the reaction especially interesting as amine groups are highly abundant in proteins and other biomolecules, which can via this route be directly immobilized. A nice feature of click chemistry reactions is their orthogonality, ${ }^{41}$ i.e. 
they occur without interfering with other reactions. It could be interesting to create functional antifouling polymer brushes that are able to incorporate different types of recognition units on one surface. Dual (or more) functionality can be either introduced within the same monomer or by the copolymerization of separately functionalized monomers. Examples of combinations are SuFEx with strain-promoted oxidation-controlled cyclooctyne-1,2-quionone cycloaddition (SPOCQ) or CuAAC (see Scheme 7.2), thiol-ene with CuAAC or CuAAC with a norbornenetetrazine combination. ${ }^{41-42}$

A

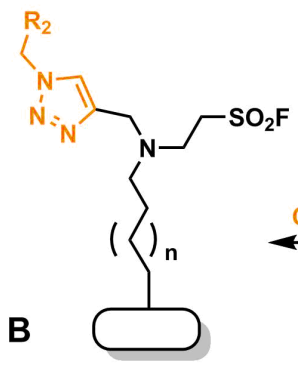

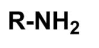
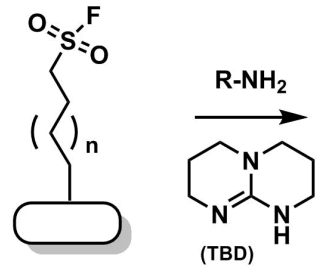

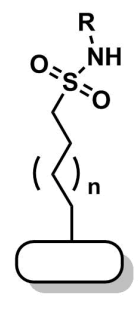

CUAAC

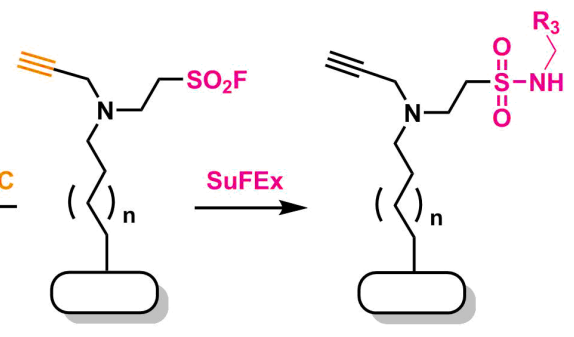

Scheme 7.2 General scheme of A) surface-bound SuFEx with amines and B) dual functionalization via CuAAC and SuFEx.

Next to the copolymerization of functional monomers, top functionalization of zwitterionic polymer brush chain ends provides another strategy to immobilize recognition units while retaining a fully zwitterionic brush. Previous studies ${ }^{19,43-44}$ (and also the work described in chapter 3) have shown that it is possible to introduce functionality via this method. In chapter 2 we compared our 3D-functionalization of azido-SB brushes with the top functionalization of SB brushes. We were, however, not able to provide evidence that the top functionalization reactions were successful at all (no characteristic azide signals in XPS and IR, nor avidin binding in reflectometry measurements). We hypothesize that the success of this mode of functionalization is to a large degree dependent on the thickness and density of the brush: as the brush becomes thicker more of the chain ends will get buried inside the brush. ${ }^{45}$ The brushes described in chapter 2 were $>35 \mathrm{~nm}$ in thickness, and were most likely too thick for successful top functionalization. Besides, ATRP of hydrophilic monomers in aqueous solutions is challenging as the polymerization is so fast that it limits the controlled nature of the polymerization and thereby increases the chance of chain termination (i.e. losing the available 
Br sites). ${ }^{46}$ Taken together, the copolymerization of functional zwitterionic monomers provides probably the best strategy to obtain surfaces with romantic character.

\subsection{Chapter 3: Specific Binding on Antifouling Zwitterionic Polymer-Coated Microbeads as Measured by Flow Cytometry}

Particles of various sizes are used for a wide range of different applications, including drug delivery, imaging, cell sorting and biosensing. ${ }^{47-49}$ Like for flat surfaces, fouling on the surface of the particles can strongly compromise their performance. In chapter 3 we described how zwitterionic antifouling polymers can be effectively introduced onto microbeads via SIATRP. The brushes were functionalized via the top functionalization of the polymer chains to incorporate biotin and mannose binding sites. The beads were shown to generically repel proteins from a $10 \%$ serum solution while being able to effectively capture streptavidin or ConA from this solution, respectively. Flow cytometry was used as read-out system and proved to be a powerful tool to evaluate the non-specific as well as specific binding of proteins on thousands of beads simultaneously. The outcomes and procedures described in chapter 3 were used as a foundation for the research described in chapter 4, 5 and 6 .

\subsubsection{Beads}

Initially we selected three types of amine-functionalized beads: Dynabeads M-270 amine (Life Technologies), ReliZyme beads (type EA403/M, Resindion) and fluorescent, amine-modified polystyrene beads (Sigma-Aldrich). We aimed to immobilize an ATRP initiator onto those beads using 2-bromo-2-methylpropionic acid and EDC/NHS chemistry. The amount of initiator that we could incorporate, also when using other coupling reagents, did not fulfill our needs for high-density polymer brushes. We hypothesized that this was caused by the steric hindrance experienced by the reagents at the surface. In agreement with this hypothesis, the reaction of an acid bromide ( $\alpha$-bromoisobutyryl bromide) in dry DCM, was found to be much more effective and thus formed a good starting point for SI-ATRP on both the Dynabeads and ReliZyme beads. The polystyrene beads could not be functionalized via this procedure as they are unstable in organic solvents. We continued our further studies with the Dynabeads because of their monodispersity, correct size for flow cytometry, and their excellent stability in both organic and aqueous media. In addition, due to their magnetic core, Dynabeads are easily separated from reaction mixtures using a magnet.

\subsubsection{Applications of antifouling beads}

Antifouling zwitterionic polymer-coated beads have a wide range of applications. Three different applications of antifouling microbeads have been discussed within this thesis: 1) a bead-based platform to study antifouling coatings (chapter 4), 2) their use in IP-MS procedures to improve 
sensitivity and specificity in the identification of protein-protein interactions (chapter 5), and 3 ) the use of antifouling coatings to evade or specifically induce phagocytic take-up by antigenpresenting cells (chapter 6). However, there are numerous other interesting possibilities. In theory, polymer brushes can be grown from the surface of any type of particle as long as an initiator molecule can be efficiently installed (at relatively high density), i.e. the coatings can be readily implemented for a wide range of applications. Two additional applications are described below.

Hydrophilic interaction chromatography (HILIC) is a relatively new method to effectively separate polar compounds, such as nucleic acids, carbohydrates and proteins, on a polar stationary phase. The polar compounds have higher affinity to the column and are therefore more strongly retained than non-polar compounds, enabling better separation. Several studies have reported the use of zwitterionic polymer-coated microbeads as a stationary phase for HILIC. . $^{50} 52$ In fact, these are among the few studies in which microbeads were used with a zwitterionic coating prepared via the grafting-from approach using living polymerization techniques such as ATRP and RAFT. It is of interest to compare the different types of antifouling materials for their performance in HILIC. The incorporation of functionality to the beads is desired for their use in affinity chromatography or affinity-based purification. ${ }^{53}$ Affinity chromatography is the combination of chromatography with biologically-related binding agents, or 'affinity ligands', that are immobilized on the stationary phase. Examples are the immobilization of streptavidin to purify biotinylated compounds, immobilization of Protein A/G to purify immunoglobulins, or nucleic acids to trap RNA or DNA via the hybridization of complementary strands. Yu et al. ${ }^{54}$ reported the preparation of PEG-based microspheres on which protein G was immobilized to purify IgG antibodies. However, the purification efficiency from complex solutions was not investigated. It must be noted that there is little difference, in terms of bead design and purification mechanism, between affinity chromatography and immunoprecipitations. Consequently, considerations (as discussed in chapter 5 and below) for applying antifouling beads in IP-MS do also apply here. Immunoprecipitations refer to protein antigens purified by means of antibodies, whereas the interactions employed in affinity chromatography are not limited to the use of antibodies.

Flow cytometric bead-based assays, such as cytometric bead arrays (CBA), are commonly used for biomolecule detection as they require small sample volumes, enable multiplexing, and allow for the quick and automated analysis of a large number of samples. ${ }^{49,55-56}$ The development towards improved bead-based assays follows the same trajectory as for other applications that face the implications of fouling. There are various studies that show improved performances of the microbeads by coating them with $\mathrm{PEG},{ }^{57-58}$ and several examples that incorporate zwitterionic moieties have also been reported. ${ }^{59-60}$ However, coatings using zwitterionic polymer brushes installed via the grafting-from approach are scarce. The beads developed in chapter 3 can be readily applied in existing protocols due to their compatibility for flow cytometry measurements. To enable multiplex assays, it would be beneficial to use (or develop) color-encoded beads ${ }^{56,61}$ as this allows the simultaneous measurement of different antigens within the same sample. Evidently, it is of great interest to determine the sensitivity that can be reached using the antifouling beads. 


\subsubsection{Romantic beads}

In chapter 3, functionalization of zwitterionic polymer-coated beads was achieved via top functionalization. It provides a versatile approach for brushes that are not too thick, in which the halogen atom at the chain ends can be converted into a wide range of functional groups. For instance, as shown in chapter 3 , the beads can be functionalized by substitution of the terminal halogen atom by a primary amine, which allows for the incorporation of a functional groups such as alkenes or alkynes. ${ }^{62}$ Difficulties may arise when organic solvents need to be used (as was needed to dissolve the fluorinated amino compound, see chapter 3, Scheme 3.1), because the zwitterionic polymer-coated beads tend to aggregate under these conditions. Converting the halogen atom into an azide moiety is therefore preferred: this reaction is performed in PBS in which the zwitterionic beads are most easily handled. Besides, azide functionalities give highly characteristic signals, both in IR and XPS, which enable monitoring of the progress of the surface reaction, and they allow for subsequent click chemistry reactions. However, the aforementioned drawbacks to top functionalization also apply to the polymer-coated beads described in chapter 3: the signals are relatively low and depend on the brush thickness and density. The generally applicable copolymerization of the azido-SB (or other functionalized zwitterionic monomers), as introduced in chapter 2, is therefore preferred, and was for this reason used in chapter 4,5 and 6.

\subsection{Chapter 4: Systematic Comparison of Zwitterionic and Non-Zwitterionic Antifouling Polymer Brushes on a Bead-Based Platform}

Despite numerous studies on the use of zwitterionic antifouling coatings there is still a need for systematic, comprehensive studies to reveal the exact relationship between the monomer structure and antifouling performance. In chapter 4 we evaluated the antifouling properties of five different polymer coatings on a bead-based platform (as was presented in chapter 3). For, as far as we know, the first time a direct comparison was made between a sulfobetaine with two and three methylene groups between opposite charges (SBMAA-2 and SBMAA-3, respectively) and a carboxybetaine with a carbon spacer length of two methylene groups (CBMAA-2). Flow cytometry results showed that (SBMAA-2) performs better in terms of antifouling than p(SBMAA-3), and that CBMAA-2 outperforms both sulfobetaines. This underlines that both carbon spacer length and the nature of the anionic group have an influence on the antifouling capacity. Interestingly, the non-zwitterionic p(HPMAA) performed equally well or better than all the tested zwitterionic coatings. Based on the obtained results the following ranking was made (with HPMAA being the best): HPMAA $\geq$ CBMAA-2 $\approx$ PCMA-2 $>$ SBMAA-2 $>$ SBMAA-3 $>$ non-modified beads. 


\subsection{SPR versus beads}

Different type of techniques are available that can be used to study the antifouling performance of surface coatings, of which SPR is probably the most frequently used method. ${ }^{20,63-65}$ SPR is a sensitive label-free sensing technique that allows for quantitative in real-time monitoring of protein adsorption, making it a very powerful tool to evaluate antifouling performance. However, we encountered various experimental challenges while evaluating our SBMAA-3 and SBMAA-2 monomers with SPR. Although SBMAA-2 outperformed SBMAA-3 in all cases, it was troublesome to obtain sensorgrams of high quality. For instance, a negative drift in the SPR signal was seen in most of the cases, of which the slope was not only changing over time but also often different for the individual channels on the same chip. To improve the results, the polymer brushes were allowed to swell/equilibrate by flowing for $3000 \mathrm{sec}$ with PBS buffer before injecting the protein samples. Although this improved the results considerably, even after overnight equilibration a drift in signal was still present. The use of a reference channel in which only PBS was injected could also not completely resolve the issue (probably because the Biacore 3000 system does not allow for the simultaneous injection of two different solutions in two different channels). The drift in signal might be caused by the slow desorption of polymer brushes. The initiator molecules were attached on the SPR gold chip via the self-assembled monolayer (SAM) formation of free thiols, which are known to have limited stability. ${ }^{7}$ The desorption of polymer brushes during antifouling studies has to our knowledge, however, not yet been reported. Another complicating factor was a sharp negative dip that was observed in the sensorgrams right after switching back from serum solution to PBS, which hampered the analysis of the sensorgrams. The signal slowly (> $15 \mathrm{~min}$ ) stabilized with running PBS buffer. We hypothesized that the negative dip was caused by a difference in salt concentration between buffer and serum solution. Dialysis of the serum solution against the PBS buffer largely prevented the presence of the dip, confirming our hypothesis. Noteworthy, sensorgrams on bare gold did lead to straight baselines and high quality sensorgrams (without dip after sample injection), confirming proper functioning of the SPR apparatus. We also observed that thorough cleaning procedures (using aqueous SDS and $\mathrm{NaOH}$ solutions) were required after each measurement to prevent increased fouling in following runs. Taken all measures together, a single SPR run became very time-consuming and still only resulted in sensorgrams of moderate quality. It must be noted that part of the issues described here might be caused by the specific setup used. For this study a Biacore 3000 was used, which is a frequently used high-performance SPR machine. It is, however, designed for premade sensor chips (usually carboxymethylated dextran-coated gold chips: CM5), while for our purpose, separate chips were modified and glued by hand in the chip holder. This required dried polymer brush-coated gold chips, which might have caused part of the aforementioned challenges. On the other hand, bare gold chips that were mounted in the same way resulted in good sensorgrams.

The difficulties faced using SPR prompted us to use beads to evaluate the antifouling performance of the different monomers. Using beads has several advantages. Firstly, it is fast: many beads can be made at once and flow cytometry measures each sample, containing thousands of beads, within a minute. Secondly, many beads are evaluated with every single measurement 
which is in strong contrast with the SPR chips in which only one surface can be measured at a time, and this manifold allows for reliable statistics. Thirdly, the bead-based platform allows for the determination of both specific as well as non-specific protein binding within the same sample. Also considering the observed degradation of the p(CBMAA-2) brushes, we anticipated that a measurement technique that is not influenced by possible mass loss of the antifouling brushes would be more trustworthy. The drawback of using the beads is that the thickness of the antifouling is difficult to measure and that labeled proteins or serum needs to be used, which might influence the protein adsorption process.

The beads, as well as SPR, do not give additional information on the behavior of polymer brushes such as their swelling or state of hydration. Other techniques like neutron reflectivity ${ }^{66}$ and quartz crystal microbalance with dissipation monitoring (QCM-D) ${ }^{67}$ that also provide information about the hydration and swelling of the brushes are highly valuable to deepen the understanding of the antifouling capacity of different polymer brushes. It is of interest to validate our hypothesis on the differences between the swelling behavior of poly(SBMAA-3) and poly(SBMAA-2) brushes under different ionic strength using these techniques.

As many beads can be prepared at the same time and samples can be efficiently measured, the bead-based platform would be highly suitable to investigate the influence of different additives/components on the antifouling properties of the polymer brushes. For example, the influence of detergents, which are often used in bio-assays and which have been shown to influence the swelling of SB polymer brushes, warrants further study. ${ }^{68}$ The presence of nucleic acids, membrane fragments or lipids, which may be present in biological fluidics, could also influence the antifouling performance. Detailed insight in their effect could help to understand the fouling process and improve current laboratory protocols that use antifouling materials.

\subsubsection{Molecular structure-function relationships}

The results obtained in chapter 4 resulted in the following ranking based on their antifouling performance: HPMAA $\geq$ PCMA-2 $\approx$ CBMAA-2 $>$ SBMAA-2 $>$ SBMAA-3 $>>$ non-modified beads. It is evident that all polymer brushes were able to effectively repel BSA from a singleprotein solution and greatly reduced the amount of fouling when exposed to $10 \%$ serum as compared to non-modified beads. Beads incubated in 10\% serum all showed detectable protein adsorption, which allowed comparison of the different polymer brushes: the differences between the tested sulfobetaines and other monomers was clearly visible. This underlines the high discriminating power of the bead-based platform. However, the approach taken did not allow to fully differentiate between the p(HPMAA), p(PCMA-2) and p(CBMAA-2) brushes. Evaluation of the antifouling performance with less diluted serum could possibly yield more pronounced differences between those monomers, as higher concentrations typically result in higher fouling. ${ }^{65,}{ }^{69}$ In contrast, our SPR results showed larger differences between SBMAA-2 and SBMAA-3 with more dilute solutions. A series of different serum solutions with varying concentration would therefore be most appropriate in an attempt to make the differences between the monomers more clear. 
Our results showed that SBMAA-2 performs better than SBMAA-3 using a 10\% serum solution, while other studies have shown that CBMAA-2 performs better than CBMAA-3 and CBMAA-5. ${ }^{20 \text {, }}$ ${ }^{70}$ These results do suggest that the shorter the chain length the better the antifouling properties and therefore that a spacer with a single methylene group would be the ultimate choice. However, Zhang et al. ${ }^{70}$ and Vaisocherova et al. ${ }^{20}$ have shown that CBMAA-1 and CBAA-1, respectively, perform equally well as the CBMAA-2/CBAA-2 monomers (all below/near the limit of detection by their SPR instrument in their experimental setup) under physiological conditions, but less well at low $\mathrm{pH}$ or ionic strength. It might be that differences become visible when the polymer brushes are exposed to more challenging incubation conditions. The degradation that we and others observed for CBMAA-2 materials ${ }^{71-73}$ is likely to be absent for the CBMAA-1 monomer as it does not allow for a Hofmann elimination (loss of $\mathrm{C}_{2} \mathrm{H}_{3} \mathrm{COO}^{-}$), suggesting that CBMAA-1 might be the better choice. SBMAA-1 has not been part of any systematic study (as far as we are aware of). A thorough study comparing SBMAA-1, SBMAA-2, SBMAA-3, CBMAA-1 and CBMAA-2 for their stability and antifouling performance under stringent conditions, such as using $100 \%$ blood plasma or serum with long exposure times, is therefore desired.

Despite the frequent (commercial) use of PCMA-2, not many systematic antifouling studies have been performed using phosphocholines. It has been shown for other types of monomers that (meth)acrylamides yield better antifouling brushes than their (meth)acrylate counterparts. ${ }^{70}$, ${ }^{74}$ Whether the PCMAA-2 performs better than PCMA-2 is currently unknown, but this is of high interest as better antifouling properties are to be expected. Besides, only then a direct comparison can be made with the CBMAA-2 and SBMAA-2 monomers. Simulation studies have shown that the CSL has limited effect on the hydration properties of the quaternary ammonium cation, but it is of great influence on the sulfonate and carboxylate anions..$^{75-76}$ It is however, unknown whether the CSL between opposite charges has an effect in the case of phosphocholines.

The aforementioned choline phosphate (CP) zwitterionic monomers are also interesting candidates. They are prepared via a one-step ring-opening reaction and can be functionalized. ${ }^{77-78}$ Interestingly, CP-based materials have been shown to resist protein adsorption while they strongly adsorb to cell membranes. This was explained by the interaction between the $\mathrm{CP}$ dipole and the dipole of the PC lipid head groups that are present in the cell membrane. ${ }^{77,79}$ This has interesting implications for drug delivery and tissue engineering. Recently, Li et al. ${ }^{80}$ reported that CP-based liposomes show better protein resistance and prolonged circulating time in mice as compared to PC-based liposomes. The CP liposomes did not show affinity for PC membranes, which is in contrast with the earlier reports on CP materials. ${ }^{77,79}$ The authors explained this by the rigid liposome structure that prevents the orientation of the CP moieties towards the PC groups within the membranes of cells (or PC liposomes). Taken together, a detailed comparison, both in terms of protein resistance and cell adhesion, between polymer brushes grown via SI-ATRP of PC and CP derivatives is desired.

Our data and simulations studies ${ }^{81}$ suggest that the ability to repel proteins is related to how strong water molecules are bound to the polymer brush, and not to the number of water molecules 
that are bound. Hydration as a result of hydrogen-bonded solvation is considered to be weaker than the hydration observed for ionic solvation. ${ }^{82-83}$ Following this reasoning, based on the chemical structure of HPMAA, it should perform considerably less well than the zwitterionic monomers. Yet, the excellent performance of HPMAA observed by us and others ${ }^{64,84}$ cannot be explained in this way. The complexation energies we found for the hydrogen bonds between a water molecule and HPMAA were considerably high $(6-12 \mathrm{kcal} / \mathrm{mol})$ and might therefore explain the excellent performance of HPMAA. More detailed simulation studies could provide a better understanding of the excellent performance of a simple hydroxyl acrylamide and could guide the design of novel and superior monomers. Whether a combination of zwitterionic properties together with the ability to form hydrogen bond bridges (two hydrogen bonds between one water molecule and one monomer) will increase the antifouling performance, remains to be elucidated. That is, it remains an intriguing - yet so far unanswered - question whether the introduction of a hydroxyl group in between the amide and ammonium group (same position as in HPMAA) will lead to improved hydration and thereby antifouling capacity (see Scheme 7.3 for several proposed structures). In addition, it is of interest to investigate the influence of the methyl group in the HPMAA monomer as HEMA and HEAA (see SScheme 7.4) have also been shown to yield excellent antifouling properties, ${ }^{74}$ and whether introducing multiple hydroxyl groups would have a beneficial effect on their antifouling performance (see proposed structure in Scheme 7.4).<smiles>C=C(C)C(=O)NCC(O)C[N+](C)(C)CCC(=O)[O-]</smiles><smiles>C=C(C)C(=O)NCCCOP(=O)([O-])OCC[N+](C)(C)C</smiles><smiles>C=C(C)C(=O)NCC(O)C[N+](C)(C)CCCS(=O)(=O)[O-]</smiles><smiles>C=C(C)C(=O)NCC(O)COP(=O)([O-])OCC[N+](C)(C)C</smiles>

Scheme 7.3 Proposed novel monomers, new elements are indicated in green.

Recently, there has been a great interest in understanding the effect of salt on the properties of zwitterionic polymer brushes. ${ }^{66,85-89} \mathrm{SB}$ polymer brushes have been shown to be especially responsive to the presence of salt, ${ }^{76}$ which can be explained by the strong inter/intra-chain interactions within the SB brushes due to the near-equal charge density of the quaternary ammonium cation and the sulfonate anion. Notably, these inter/intra-chain associations have been utilized to create antifouling surface coatings that show self-healing properties upon the addition of water. ${ }^{90}$ The inter/intra-chain interactions can be disrupted upon addition of salt as the ions can screen the charges of the zwitterions, preventing them to interact with one another. As a result, upon addition of salts the SB polymer brushes swell and increase their state of hydration, which leads to an improved antifouling performance. ${ }^{67} \mathrm{CB}$ polymer brushes on the other hand, bear charges with quite different charge densities leading to fewer 
associations and are hence less responsive to salt. ${ }^{91}$ The exact effect of salt on the polymer properties, is dependent on monomer structure, ${ }^{66-67,85}$ density and thickness of the brushes, ${ }^{92}$ but also on ionic strength as well as on the type of ions that are added. ${ }^{85,88}$ This results in a complex interplay of different effects which are not yet entirely understood. Some generalized observations are that anions have more effect than cations, ${ }^{66-67,85}$ and that monovalent anions like chlorine and bromine have a positive effect on the extent of brush swelling while divalent ions such as $\mathrm{SO}_{4}{ }^{2-}$ have adverse effects and elicit more complex behavior. ${ }^{66-67,85,88}$ The studies towards a better understanding of the effects of salt on the antifouling behavior of zwitterionic polymer coatings have primarily focused on the effect of $\mathrm{Cl}^{-}, \mathrm{Br}^{-}$and $\mathrm{SO}_{4}{ }^{2-}$, which overlooks the ions that are predominantly present in biological fluids and PBS buffers, like $\mathrm{HCO}_{3}^{-}, \mathrm{Ca}^{2+}$, $\mathrm{Mg}^{2+}$ and $\mathrm{HPO}_{4}{ }^{2-} / \mathrm{H}_{2} \mathrm{PO}_{4}^{-}$. Studying the influence of those ions in mixtures at physiological concentrations could assist in understanding discrepancies observed between brushes evaluated in single ion-solutions and that of brushes exposed to blood serum or plasma (as with our comparison between SBMAA-2 and SBMAA-3) and could potentially lead to the use of specialized buffers for enhanced biosensing performance.

\subsection{Chapter 5: Specific Protein Identification by Immunoprecipitation-Mass Spectrometry Using Antifouling Microbeads}

Immunonoprecipitation followed by mass spectrometry (IP-MS) has been shown to be a powerful bead-based tool to study protein-protein interactions. ${ }^{93-94}$ However, it is often challenging to differentiate between true protein interactors and proteins that bind nonspecifically. In chapter 5 the use of antifouling beads to reduce background signals in IPMS experiments was presented. Functionalization of the zwitterionic polymer brushes via the copolymerization with azido-SB monomers (chapter 2) enabled the immobilization of antibodies via click chemistry. A generic antifouling anti-GFP bead was prepared which showed a similar amount of GFP capture as commercial beads, yet with much less binding of contaminating proteins. Our anti-GFP beads showed efficient capture of GFP-fusion proteins from cell lysate and were able to co-purify known interaction partners of the used GFP-tagged bait protein. This illustrates the high potential of those beads to identify unknown proteinprotein interactions.

\subsubsection{Preparation of IP-MS beads}

The generic antifouling anti-GFP beads described in chapter 5 showed reduced binding of contaminating proteins in both flow cytometry and IP-MS measurements, as compared to both non-modified beads and commercially available anti-GFP beads that are widely used for immunoprecipitations. However, a considerable amount of fouling was still observed when the beads were exposed to a $10 \%$ serum solution. Optimization of the zwitterionic coating preparation as well as the antibody attachment will aid in reaching the full potential of antifouling beads in IP-MS procedures. Further optimization of polymerization time, azido- 


\section{Reported monomers}<smiles>C=C(C)C(=O)NCC(C)O</smiles>

HPMA<smiles>C=C(C)C(=O)OCCO</smiles>

HEMA<smiles>C=CC(=O)NCCO</smiles>

HEAA

\section{Proposed novel monomers}<smiles>C=C(C)C(=O)NCC(O)CO</smiles><smiles>C=C(C)C(=O)NCCO</smiles>

Scheme 7.4 Chemical structures of reported and novel monomers as described in the text.

SB percentage, BCN-NHS linker to antibody ratio, antibody concentration and reaction time during antibody immobilization could yield improved antifouling performance of the polymer coatings. In chapter 4 it was shown that the standard SBMAA-3 monomer is not the best monomer. Copolymerization of the azido-SB with other monomers such as SBMAA-2, HPMA or PCMAA-2 might therefore be considered. The functionalized PC and CP monomers (Scheme 7.1) are also valuable candidates to be tested. Our data showed that the immobilization of a 'standard' IgG antibody (TA99) lead to more fouling than the attachment of the $\mathrm{V}_{\mathrm{H}} \mathrm{H}$ domain of a camelid antibody (anti-GFP), probably caused by the smaller size of the latter. The antifouling properties are imparted by the zwitterionic polymer brushes, anything else on the beads is likely to increase the amount of fouling. Oriented antibody immobilization could lead to a further improvement of the beads. The use of covalently bound oriented llama antibodies $\left(\mathrm{V}_{\mathrm{H}} \mathrm{H}\right.$ domains) has been shown to improve sensor sensitivity up to a factor 800 as compared to the same antibody randomly oriented..$^{95}$ As oriented immobilized antibodies are more effective in binding their target, it is expected that less antibody is needed per IP-MS experiment, which also could improve the IP-MS outcome by better suppressing non-specific binding. A significant drawback of this method is that oriented attachment of the llama antibodies requires protein engineering. IgG antibodies can also be used for oriented immobilization, for example, using boronic acids targeting the $\mathrm{N}$-glycans on the Fc regions from the antibodies. ${ }^{96-97}$

\subsubsection{Immunoprecipitations}

In chapter 5 we showed the preparation of a generic anti-GFP bead that was used to capture MBD3-GFP and its known interaction partners. Evidently, it would be of interest to use the novel antifouling anti-GFP-functionalized beads to discover new protein-protein interactions. Analogously to generic anti-GFP beads, additional generic beads directed against other epitope tags, such as c-myc, FLAG, and His-tag, ${ }^{98}$ can also be prepared to expand the usability of the beads. It must be noted that even without MS analysis, these generic beads can be of great value for the purification of tagged proteins. ${ }^{99}$ The immobilization of streptavidin is also valuable as 
it allows for the binding of a wide variety of biotinylated antibodies, and can hence be used to capture a wide range of antigens. Within this thesis we have shown the successful attachment of biotin, mannose, camelid anti-GFP nanobody, mouse IgG TA99 antibody and chicken OVA to the antifouling beads. It is therefore likely that other recognition units can also be readily incorporated.

Once recognition units are immobilized on the antifouling beads, they can subsequently be used to 'precipitate' tagged or non-tagged bait proteins and study the co-purified interacting proteins by mass spectrometry. Alternatively, the beads can be used to identify unknown allergens via the immobilization of IgE antibodies. IgE antibodies help to combat parasites in healthy individuals but also play a pivotal role in the response to allergens. ${ }^{100}$ It is therefore of interest to immobilize IgE antibodies (or first anti-IgE antibodies to make the beads more generic) of allergic individuals to the antifouling beads and incubate them in a pool of possible allergens. MS analysis of bound biomolecules could then identify to which allergens the IgE antibodies were raised. The use of antifouling beads will aid in identifying true allergens and lower the risk of false-positive candidates.

\subsubsection{IP-MS as a tool for antifouling studies}

Besides the use of functionalized antifouling beads in IP-MS experiments to study proteinprotein interactions, the beads can also be used as a tool to study antifouling behavior. Although the major proteins that play a role in fouling have been identified, such as immunoglobulins, albumin, fibrinogen and complement factors, ${ }^{101}$ the exact mechanism and compositions of fouling proteins are poorly understood. It has been shown that serum or plasma from different individuals, age of individuals and type of species can cause differences in the amount of fouling. ${ }^{84,102}$ Besides, there have been only a few studies that focus on antifouling in solutions different than single-protein solutions or blood plasma/serum, such as urine, saliva, milk and fruit juices..$^{28,102-103}$ The bead-based platform as described within this thesis, followed by mass spectrometry analysis can provide valuable information to deepen the understanding of fouling. Recently, such a study was performed on nanoparticles incubated in cell culture media. ${ }^{104}$

\subsection{Chapter 6: Targeted Phagocytosis of Functionalized Stealth Microbeads by Macrophages}

The performance of particles for in vivo applications such as imaging and drug delivery is significantly hampered by non-specific protein adsorption as this induces phagocytic uptake by macrophages and other antigen-presenting cells. This leads to the rapid clearance of the particles from the blood stream, preventing the particles from reaching their target site. Coating the particles with an antifouling layer can significantly reduce the amount of fouling on the particles and hence suppress their phagocytic uptake. In Chapter 6 we showed a proof-ofprinciple study in which the zwitterionic polymer-coated microbeads were able to prevent phagocytosis by murine macrophages. Immobilization of ovalbumin (OVA) on the beads 
did not result in an increase in phagocytic uptake. Only when the beads were specifically targeted to the macrophages by the direct or indirect coupling of $\operatorname{IgG}_{2 \mathrm{a}}$ antibodies, for which the macrophages have a receptor, the antifouling beads were efficiently internalized. While only a first, preliminary study, our work does illustrate the great potential of zwitterionic polymer brushes for in vivo applications.

\subsubsection{Antigen presentation}

In chapter 6 we showed that antifouling beads coated with OVA were not phagocytosed, whereas beads coated with $\operatorname{IgG}_{2 \mathrm{a}}$ antibodies were efficiently taken up by macrophages. By coating the beads with antibodies, antigen-presenting cells (APCs), such as macrophages and dendritic cells, can efficiently phagocytose the beads following the binding of their Fc receptors (FCR) to the $\mathrm{Fc}_{\mathrm{C}}$ region of the antibody. The mouse $\operatorname{IgG}_{2 \mathrm{a}}$ antibody isotype can bind to activating $\mathrm{FC}$ receptors (FcgRI, FcgRIII and FcgRIV) on APCs and is the most potent IgG subclass to promote phagocytosis and upregulation of maturation markers such as CD80 and CD86. ${ }^{105}$ The enhanced phagocytosis will promote the major histocompatibility complex (MHC) presentation of beadderived peptides on the surface of the APCs. Together with the $\operatorname{IgG}_{2 a}$, induced upregulation of the costimulatory surface markers CD80 and CD86, will signal naive antigen specific T cells to become activated. ${ }^{105-106}$ The stealth characteristics of the OVA-functionalized antifouling beads can therefore be transformed into immune stimulating beads by binding them to the anti-OVA $\mathrm{IgG}_{2 \mathrm{a}}$ antibodies, ensuring effective presentation of OVA peptides. Immobilization of proteins or biomolecules that are associated with pathogens (instead of OVA) together with antibodies with $\mathrm{FC}_{\mathrm{C}}$ regions that target these activating Fc receptors, could serve as promising candidates for vaccine development. Macrophages and dendritic cells (DCs) express both activatory and inhibitory Fc $\gamma$ Rs, the balance of which helps to regulate their activation status. Immune complexes of antigen and antibody isotype that binds the inhibitory Fc $\gamma$ RIIB receptor, were shown to induce an immature/tolerogenic DC phenotype that resulted in the suppression of autoreactive T cells. ${ }^{107}$ Induction of tolerance might therefore be promoted by targeting antigen-carrying stealth beads with FcgRIIB. This could offer therapeutic applications for allergies and autoimmune diseases. Whether the antifouling polymer-coated beads will indeed improve antigen presentation or can be used to induce tolerance remains to be elucidated.

\subsubsection{In vivo applications}

The $2.8 \mu \mathrm{m}$ Dynabeads presented in Chapter 6 were used to show the potential of the SIATRP-grown zwitterionic coatings. Beads in this size range have high potential for particlebased vaccine development. ${ }^{108-109}$ In contrast, for in vivo imaging or drug delivery applications, smaller particles are more common practice (in the range of 2-300 $\mathrm{nm}^{110}$ ). The incorporation of zwitterionic units in nanoparticle systems has recently been utilized to improve drug delivery and in vivo imaging. For example, zwitterion-coated quantum dots ${ }^{111}$ and zwitterionic dendrimer-entrapped gold nanoparticles ${ }^{112}$ have been used for enhanced tumor imaging in mice, while zwitterionic micelles have been proposed as promising drug delivery carriers. ${ }^{113-115}$ 
Few studies have used SI-ATRP to coat nanoparticles, ${ }^{22,116}$ and even less have applied them for in vivo applications. It is therefore of interest to transfer the methodologies for micron-sized particles developed within this thesis to nanoparticles and investigate their performance in both in vitro and in vivo experiments.

On flat surfaces it has been shown that polymer coatings prepared via the grafting-from approach yield the best antifouling performances, and it is expected that this is also true for polymer brushes grown from nanoparticles. However, it is difficult to predict the differences in antifouling performance of nanoparticle with zwitterionic coatings prepared by SI-ATRP with that of zwitterionic dendrimers, nanoparticles formed by the self-assembly of diblock polymers (zwitterionic and hydrophobic block), zwitterionic micelles and zwitterionic liposomes. We have only just begun to understand the behavior of these different type of nanoparticles in vivo, and further studies on these various types of nanoparticles are needed to determine and compare their antifouling performance.

\subsubsection{The future of antifouling materials}

Antifouling coatings are being implemented in a wide variety of applications, ranging from medical implants and marine coatings to biosensing systems. ${ }^{117}$ Recently, new applications have emerged such as their use in anti-icing coatings for aviation, ${ }^{118}$ or their ability to capture, kill and release bacteria. ${ }^{119}$ Improvements, new features and novel applications are continuously being added, which will further expand the usage and scope of antifouling polymer brushes. As different applications have different requirements and as antifouling performance is dependent on many factors (type of coating, type of protein solution, temperature, ionic strength, time of exposure, etc.), a 'one fits all' antifouling material cannot fulfill all requirements. A 'fit-forpurpose' approach, in which the antifouling coating is specifically tailored to the application, is therefore in the author's view best suited. A better understanding of which antifouling material performs best in what situation, is therefore highly desired.

Antifouling coatings have high potential in many biological applications, but their implementation is often challenging. To increase the usage of zwitterionic polymer coatings in common (biology) laboratory practice, the technology should be either (further) commercialized and/ or procedures should be developed that allow for the formation of zwitterionic coatings on a common laboratory bench. High performance antifouling coatings are usually prepared by SI-ATRP, however, this method requires stringent oxygen-free laboratory procedures which are non-trivial for a non-chemist. Several adjustments have been made to make the SI-ATRP procedure less sensitive to oxygen, ${ }^{120}$ for example by the addition of reducing agents, however, ATRP remains a delicate method for which alternatives are highly appreciated.

Taken together, this thesis provides new insights and tools to efficiently introduce functionality to zwitterionic polymer brushes and to incorporate these brushes onto a bead-based platform. This thesis thereby considerably contributes to the applicability of zwitterionic polymer brushes for biomedical applications. 


\subsection{References}

1. Pujari, S. P.; Scheres, L.; Marcelis, A. T. M.; Zuilhof, H. Covalent Surface Modification of Oxide Surfaces. Angew. Chem. Int. Ed. 2014, 53, 6322-6356.

2. Du, Y.; Gao, J.Y.; Chen, T.T.; Zhang, C.; Ji, J.; Xu, Z. K. Understanding the Oxidative Stability of Antifouling Polymer Brushes. Langmuir 2017, 33, 7298-7304.

3. Quintana, R.; Gosa, M.; Janczewski, D.; Kutnyanszky, E.; Vancso, G. J. Enhanced Stability of Low Fouling Zwitterionic Polymer Brushes in Seawater with Diblock Architecture. Langmuir 2013, 29, 10859-10867.

4. Ataman, N. C.; Klok, H. A. Degrafting of Poly(Poly(Ethylene Glycol) Methacrylate) Brushes from Planar and Spherical Silicon Substrates. Macromolecules 2016, 49, 9035-9047.

5. Heideman, R.; Hoekman, M.; Schreuder, E. TriPleX-Based Integrated Optical Ring Resonators for Lab-on-a-Chip and Environmental Detection. IEEE J. Sel.Top. Quant. 2012, 18, 1583-1596.

6. Carlborg, C. F.; Gylfason, K. B.; Kazmierczak, A.; Dortu, F.; Polo, M. J. B.; Catala, A. M.; Kresbach, G. M.; Sohlstrom, H.; Moh, T.; Vivien, L.; Popplewell, J.; Ronan, G.; Barrios, C.A.; Stemme, G.; van derWijngaart, W. A Packaged Optical Slot-Waveguide Ring Resonator Sensor Array for Multiplex Label-Free Assays in Labs-on-Chips. Lab Chip 2010, 10, 281-290.

7. Bhairamadgi, N. S.; Pujari, S. P.; Trovela, F. G.; Debrassi, A.; Khamis, A. A.; Alonso, J. M.; Al Zahrani, A. A.; Wennekes, T.; AlTuraif, H. A.; van Rijn, C.; Alhamed, Y. A.; Zuilhof, H. Hydrolytic and Thermal Stability of Organic Mono Layers on Various Inorganic Substrates. Langmuir 2014, 30, 5829-5839.

8. Rosso, M.; Giesbers, M.; Arafat, A.; Schroen, K.; Zuilhof, H. Covalently Attached Organic Monolayers on $\mathrm{SiC}_{\text {and }} \mathrm{Si}_{\mathrm{x}} \mathrm{N}_{4}$ Surfaces: Formation Using UV Light at Room Temperature. Langmuir 2009, 25, 2172-2180.

9. Arafat, A.; Schroen, K.; de Smet, L. C. P. M.; Sudholter, E. J. R.; Zuilhof, H. Tailor-Made Functionalization of Silicon Nitride Surfaces. J.Am. Chem. Soc. 2004, 126, 8600-8601.

10. Nguyen, A.T.; Baggerman, J.; Paulusse, J. M. J.; van Rijn, C. J. M.; Zuilhof, H. Stable Protein-Repellent Zwitterionic Polymer Brushes Grafted from Silicon Nitride. Langmuir 2011, 27, 2587-2594.

11. Scheres, L.; Giesbers, M.; Zuilhof, H. Organic Monolayers onto Oxide-Free Silicon with Improved Surface Coverage: Alkynes Versus Alkenes. Langmuir 2010, 26, 4790-4795.

12. Liu, L. H.; Michalak, D. J.; Chopra, T. P.; Pujari, S. P.; Cabrera, W.; Dick, D.; Veyan, J. F.; Hourani, R.; Halls, M. D.; Zuilhof, H.; Chabal, Y. J. Surface Etching, Chemical Modification and Characterization of Silicon Nitride and Silicon Oxide-Selective Functionalization of $\mathrm{Si}_{3} \mathrm{~N}_{4}$ and $\mathrm{SiO}_{2}$. J. Phys. Condens. Matter 2016, 28, 094014.

13. Stine, R.; Cole, C. L.; Ainslie, K. M.; Mulvaney, S. P.; Whitman, L. J. Formation of Primary Amines on Silicon Nitride Surfaces: A Direct, Plasma-Based Pathway to Functionalization. Langmuir 2007, 23, 4400-4404.

14. Jiang, H.; Xu, F. J. Biomolecule-Functionalized Polymer Brushes. Chem. Soc. Rev. 2013, 42, 3394-426.

15. Zoppe, J. O.; Ataman, N. C.; Mocny, P.; Wang, J.; Moraes, J.; Klok, H. A. Surface-Initiated Controlled Radical Polymerization: State-of-the-Art, Opportunities, and Challenges in Surface and Interface Engineering with Polymer Brushes. Chem. Rev. 2017, 117, 1105-1318.

16. Chalarca, C. F. S.; Emrick, T. Reactive Polymer Zwitterions: Sulfonium Sulfonates. J. Polym. Sci., Part A: Polym. Chem. 2017, 55, 83-92.

17. Chou,Y. N.;Wen,T. C.; Chang,Y. Zwitterionic Surface Grafting of Epoxylated Sulfobetaine Copolymers for the Development of Stealth Biomaterial Interfaces. Acta Biomater. 2016, 40, 78-91.

18. Huang, C. J.; Li, Y.; Jiang, S. Zwitterionic Polymer-Based Platform with Two-Layer Architecture for Ultra Low Fouling and High Protein Loading. Anal. Chem. 2012, 84, 3440-3445.

19. Nguyen, A. T.; Baggerman, J.; Paulusse, J. M. J.; Zuilhof, H.; van Rijn, C. J. M. Bioconjugation of Protein-Repellent Zwitterionic Polymer Brushes Grafted from Silicon Nitride. Langmuir 2012, 28, 604-610. 
20. Vaisocherova, H.; Zhang, Z.; Yang, W.; Cao, Z. Q.; Cheng, G.; Taylor, A. D.; Piliarik, M.; Homola, J.; Jiang, S.Y. Functionalizable Surface Platform with Reduced Nonspecific Protein Adsorption from Full Blood Plasma-Material Selection and Protein Immobilization Optimization. Biosens. Bioelectron. 2009, 24, 1924-1930.

21. Lisalova, H.; Brynda, E.; Houska, M.; Visova, I.; Mrkvova, K.; Song, X. C.; Gedeonova, E.; Surman, F.; Riedel, T.; PopGeorgievski, O.; Homola, J. Ultralow-Fouling Behavior of Biorecognition Coatings Based on Carboxy-Functional Brushes of Zwitterionic Homo- and Copolymers in Blood Plasma: Functionalization Matters. Anal. Chem. 2017, 89, 3524-3531.

22. Yang, W.; Zhang, L.; Wang, S. L.; White, A. D.; Jiang, S. Y. Functionalizable and Ultra Stable Nanoparticles Coated with Zwitterionic Poly(Carboxybetaine) in Undiluted Blood Serum. Biomaterials 2009, 30, 5617-5621.

23. Brault, N. D.; White, A. D.; Taylor, A. D.; Yu, Q. M.; Jiang, S.Y. Directly Functionalizable Surface Platform for Protein Arrays in Undiluted Human Blood Plasma. Anal. Chem. 2013, 85, 1447-1453.

24. Booth, M. A.; Kannappan, K.; Hosseini, A.; Partridge, A. In-Depth Electrochemical Investigation of Surface Attachment Chemistry Via Carbodiimide Coupling. Langmuir 2015, 31, 8033-8041.

25. Palazon, F.; Montenegro Benavides, C.; Léonard, D.; Souteyrand, É.; Chevolot, Y.; Cloarec, J. P. Carbodiimide/NHS Derivatization of COOH-Terminated SAMs: Activation or Byproduct Formation? Langmuir 2014, 30, 4545-4550.

26. Sam, S.; Touahir, L.; Salvador Andresa, J.; Allongue, P.; Chazalviel, J. N.; Gouget-Laemmel, A. C.; Henry de Villeneuve, C.; Moraillon, A.; Ozanam, F.; Gabouze, N.; Djebbar, S. Semiquantitative Study of the EDC/NHS Activation of Acid Terminal Groups at Modified Porous Silicon Surfaces. Langmuir 2010, 26, 809-814.

27. Wang, C.; Yan, Q.; Liu, H.-B.; Zhou, X.-H.; Xiao, S.-J. Different EDC/NHS Activation Mechanisms between PAA and PMAA Brushes and the Following Amidation Reactions. Langmuir 2011, 27, 12058-12068.

28. Vaisocherova, H.; Sevcu, V.; Adam, P.; Spackova, B.; Hegnerova, K.; Pereira, A. D.; Rodriguez-Emmenegger, C.; Riedel, T.; Houska, M.; Brynda, E.; Homola, J. Functionalized Ultra-Low Fouling Carboxy- and Hydroxy-Functional Surface Platforms: Functionalization Capacity, Biorecognition Capability and Resistance to Fouling from Undiluted Biological Media. Biosens. Bioelectron. 2014, 51, 150-157.

29. Hu, G. J.; Emrick, T. Functional Choline Phosphate Polymers. J.Am. Chem. Soc. 2016, 138, 1828-1831.

30. Song, L. J.; Zhao, J.; Luan, S. F.; Ma, J.; Liu, J. C.; Xu, X. D.; Yin, J. H. Fabrication of a Detection Platform with Boronic-AcidContaining Zwitterionic Polymer Brush. ACS Appl. Mater. Interfaces 2013, 5, 13207-13215.

31. Lobb, E. J.; Ma, I.; Billingham, N. C.; Armes, S. P.; Lewis, A. L. Facile Synthesis of Well-Defined, Biocompatible Phosphorylcholine-Based Methacrylate Copolymers Via Atom Transfer Radical Polymerization at 20 Degrees C. J.Am. Chem. Soc. 2001, 123, 7913-7914.

32. Hong, V.; Presolski, S. I.; Ma, C.; Finn, M. G. Analysis and Optimization of Copper-Catalyzed Azide-Alkyne Cycloaddition for Bioconjugation. Angew. Chem. Int. Ed. 2009, 48, 9879-9883.

33. Baskin, J. M.; Prescher, J. A.; Laughlin, S. T.; Agard, N. J.; Chang, P.V.; Miller, I. A.; Lo, A.; Codelli, J. A.; Bertozzi, C. R. CopperFree Click Chemistry for Dynamic in Vivo Imaging. Proc. Natl.Acad. Sci. U. S.A. 2007, 104, 16793-16797.

34. Dommerholt, J.; Schmidt, S.; Temming, R.; Hendriks, L. J. A.; Rutjes, F. P. J.T.; van Hest, J. C. M.; Lefeber, D. J.; Friedl, P.; van Delft, F. L. Readily Accessible Bicyclononynes for Bioorthogonal Labeling and Three-Dimensional Imaging of Living Cells. Angew. Chem. Int. Ed. 2010, 49, 9422-9425.

35. Kolb, H. C.; Finn, M. G.; Sharpless, K. B. Click Chemistry: Diverse Chemical Function from a Few Good Reactions. Angew. Chem. Int. Ed. 2001, 40, 2004-2021.

36. Manova, R. K.; Pujari, S. P.; Weijers, C. A.; Zuilhof, H.; van Beek, T. A. Copper-Free Click Biofunctionalization of Silicon Nitride Surfaces Via Strain-Promoted Alkyne-Azide Cycloaddition Reactions. Langmuir 2012, 28, 8651-8663.

37. Sen, R.; Escorihuela, J.; Smulders, M. M. J.; Zuilhof, H. Use of Ambient Ionization High-Resolution Mass Spectrometry for the Kinetic Analysis of Organic Surface Reactions. Langmuir 2016, 32, 3412-3419.

38. Dong, J. J.; Krasnova, L.; Finn, M. G.; Sharpless, K. B. Sulfur(VI) Fluoride Exchange (SuFEx): Another Good Reaction for Click Chemistry. Angew. Chem. Int. Ed. 2014, 53, 9430-9448. 
39. Yatvin, J.; Brooks, K.; Locklin, J. Sufex on the Surface: A Flexible Platform for Postpolymerization Modification of Polymer Brushes. Angew. Chem. Int. Ed. 2015, 54, 13370-13373.

40. Gahtory, D.; Sen, Rickdeb.; Pujari, S. P.; Li, S.; Zheng, Q.; Moses, J. E.; Sharpless, K. B.; Zuilhof, H. Quantitative and Orthogonal Formation and Reactivity of SuFEx Platforms. Eur.J. Chem. 2018, 24, 10550-10556

41. Tunca, U. Orthogonal Multiple Click Reactions in Synthetic Polymer Chemistry. J. Polym. Sci., Part A: Polym. Chem. 2014, 52, 3147-3165.

42. Brooks, K.; Yatvin, J.; McNitt, C. D.; Reese, R. A.; Jung, C.; Popik, V.V.; Locklin, J. Multifunctional Surface Manipulation Using Orthogonal Click Chemistry. Langmuir 2016, 32, 6600-6605.

43. Lee, B. S.; Lee, J. K.; Kim, W. J.; Jung,Y. H.; Sim, S. J.; Lee, J.; Choi, I. S. Surface-Initiated, Atom Transfer Radical Polymerization of Oligo(Ethylene Glycol) Methyl Ether Methacrylate and Subsequent Click Chemistry for Bioconjugation. Biomacromolecules 2007, 8, 744-7499.

44. Li, Y.; Giesbers, M.; Gerth, M.; Zuilhof, H. Generic Top-Functionalization of Patterned Antifouling Zwitterionic Polymers on Indium Tin Oxide. Langmuir 2012, 28, 12509-12517.

45. Spiliopoulos, N.; Koutsioubas, A. G.; Anastassopoulos, D. L.; Vradis, A. A.; Toprakcioglu, C.; Menelle, A.; Mountrichas, G.; Pispas, S. Neutron Reflectivity Study of Free-End Distribution in Polymer Brushes. Macromolecules 2009, 42, 6209-6214.

46. He, W.; Jiang, H.; Zhang, L.; Cheng, Z.; Zhu, X. Atom Transfer Radical Polymerization of Hydrophilic Monomers and Its Applications. Polym. Chem. 2013, 4, 2919-2938

47. Mornet, S.; Vasseur, S.; Grasset, F.; Veverka, P.; Goglio, G.; Demourgues, A.; Portier, J.; Pollert, E.; Duguet, E. Magnetic Nanoparticle Design for Medical Applications. Prog. Solid State Chem. 2006, 34, $237-247$.

48. Arruebo, M.; Fernández-Pacheco, R.; Ibarra, M. R.; Santamaría, J. Magnetic Nanoparticles for Drug Delivery. Nano Today 2007, 2, 22-32.

49. Morgan, E.;Varro, R.; Sepulveda, H.; Ember, J.A.; Apgar, J.; Wilson, J.; Lowe, L.; Chen, R.; Shivraj, L.; Agadir, A.; Campos, R.; Ernst, D.; Gaur, A. Cytometric Bead Array: A Multiplexed Assay Platform with Applications in Various Areas of Biology. Clin. Immunol. 2004, 110, 252-266.

50. Celebi, B.; Ozen, B.; Kip, C.; Tuncel, A. A New Stationary Phase for Hydrophilic Interaction Chromatography: PolyacrylateBased Hydrophilic, Monosized-Porous Beads with Zwitterionic Molecular Brushes. Chromatographia 2014, 77, 1511-1520.

51. Hemstrom, P.; Szumski, M.; Irgum, K. Atom-Transfer Radical Graft Polymerization Initiated Directly from Silica Applied to Functionalization of Stationary Phases for High-Performance Liquid Chromatography in the Hydrophilic Interaction Chromatography Mode. Anal. Chem. 2006, 78, 7098-7103.

52. Huang, G.; Xiong, Z. C.; Qin, H. Q.; Zhu, J.; Sun, Z.; Zhang,Y.; Peng, X. J.; Ou, J.J.; Zou, H. F. Synthesis of Zwitterionic Polymer Brushes Hybrid Silica Nanoparticles Via Controlled Polymerization for Highly Efficient Enrichment of Glycopeptides. Anal. Chim. Acta 2014, 809, 61-68.

53. Zhang, C.; Rodriguez, E.; Bi, C.; Zheng, X.; Suresh, D.; Suh, K.; Li, Z.; Elsebaei, F.; Hage, D. S. High Performance Affinity Chromatography and Related Separation Methods for the Analysis of Biological and Pharmaceutical Agents. The Analyst 2018, 143, 374-391.

54. Yu, L.; Shi, Z. Z.; Li, C. M. Atom Transfer Radical Polymerization to Fabricate Monodisperse Poly[Glycidyl Methacrylate-CoPoly (Ethylene Glycol) Methacrylate] Microspheres and Its Application for Protein Affinity Purification. J. Colloid Interface Sci. 2015, 453, 151-158.

55. Cook, E. B.; Stahl, J. L.; Lowe, L.; Chen, R.; Morgan, E.;Wilson, J.;Varro, R.; Chan,A.; Graziano, F. M.; Barney, N. P. Simultaneous Measurement of Six Cytokines in a Single Sample of Human Tears Using Microparticle-Based Flow Cytometry, Allergies Vs. Non-Allergies. J. Immunol. Methods 2001, 254, 109-118.

56. Meimaridou, A.; Haasnoot, W.; Noteboom, L.; Mintzas, D.; Pulkrabova, J.; Hajslová, J.; Nielen, M. W. F. Color Encoded Microbeads-Based Flow Cytometric Immunoassay for Polycyclic Aromatic Hydrocarbons in Food. Anal. Chim. Acta 2010, 672, 9-14. 
57. Lin, R.; Li, Y. C.; MacDonald, T.; Wu, H.; Provenzale, J.; Peng, X. G.; Huang, J.; Wang, L. Y.; Wang, A. Y.; Yang, J.Y.; Mao, H. Improving Sensitivity and Specificity of Capturing and Detecting Targeted Cancer Cells with Anti-Biofouling Polymer Coated Magnetic Iron Oxide Nanoparticles. Colloids Surf., B 2017, 150, 261-270.

58. Pochechueva, T.; Chinarev, A.; Bovin, N.; Fedier, A.; Jacob, F.; Heinzelmann-Schwarz, V. PEGylation of Microbead Surfaces Reduces Unspecific Antibody Binding in Glycan-Based Suspension Array. J. Immunol. Methods 2014, 412, 42-52.

59. Kim, G.; Yong, Y.; Kang, H. J.; Park, K.; Kim, S. I.; Lee, M.; Huh, N. Zwitterionic Polymer-Coated Immunobeads for BloodBased Cancer Diagnostics. Biomaterials 2014, 35, 294-303.

60. Kim, G.;Yoo, C. E.; Kim, M.; Kang, H. J.; Park, D.; Lee, M.; Huh, N. Noble Polymeric Surface Conjugated with Zwitterionic Moieties and Antibodies for the Isolation of Exosomes from Human Serum. Biocon. Chem. 2012, 23, 2114-2120.

61. Peters, J.; Thomas, D.; Boers, E.; de Rijk, T.; Berthiller, F.; Haasnoot, W.; Nielen, M. W. F. Colour-Encoded Paramagnetic Microbead-Based Direct Inhibition Triplex Flow Cytometric Immunoassay for Ochratoxin A, Fumonisins and Zearalenone in Cereals and Cereal-Based Feed. Anal. Bioanal. Chem. 2013, 405, 7783-7794.

62. Debrassi, A.; Roeven, E.; Thijssen, S.; Scheres, L.; de Vos, W. M.; Wennekes, T.; Zuilhof, H. Versatile (Bio)Functionalization of Bromo-Terminated Phosphonate-Modified Porous Aluminum Oxide. Langmuir 2015, 31, 5633-5644.

63. Guo, X. Surface Plasmon Resonance Based Biosensor Technique: A Review. J. Biophotonics 2012, 5, 483-501.

64. Rodriguez-Emmenegger, C.; Brynda, E.; Riedel, T.; Houska, M.; Subr, V.; Alles, A. B.; Hasan, E.; Gautrot, J. E.; Huck, W.T. S. Polymer Brushes Showing Non-Fouling in Blood Plasma Challenge the Currently Accepted Design of Protein Resistant Surfaces. Macromol. Rapid Commun. 2011, 32, 952-957.

65. Ladd, J.; Zhang, Z.; Chen, S.; Hower, J. C.; Jiang, S. Zwitterionic Polymers Exhibiting High Resistance to Nonspecific Protein Adsorption from Human Serum and Plasma. Biomacromolecules 2008, 9, 1357-1361.

66. Higaki,Y.; Inutsuka,Y.; Sakamaki,T.; Terayama,Y.; Takenaka, A.; Higaki, K.; Yamada, N. L.; Moriwaki,T.; Ikemoto, Y.; Takahara, A. Effect of Charged Group Spacer Length on Hydration State in Zwitterionic Poly(Sulfobetaine) Brushes. Langmuir 2017, 33, 8404-8412.

67. Wang, T.; Kou, R.; Liu, H.; Liu, L.; Zhang, G.; Liu, G. Anion Specificity of Polyzwitterionic Brushes with Different Carbon Spacer Lengths and Its Application for Controlling Protein Adsorption. Langmuir 2016, 32, 2698-2707.

68. Yang, Z.; Zhang, S.; Tarabara, V. V.; Bruening, M. L. Aqueous Swelling of Zwitterionic Poly(Sulfobetaine Methacrylate) Brushes in the Presence of Ionic Surfactants. Macromolecules 2018, 51, 1161-1171.

69. Yang, W.; Chen, S. F.; Cheng, G.; Vaisocherova, H.; Xue, H.; Li, W.; Zhang, J. L.; Jiang, S. Y. Film Thickness Dependence of Protein Adsorption from Blood Serum and Plasma onto Poly(Sulfobetaine)-Grafted Surfaces. Langmuir 2008, 24,9211 9214.

70. Zhang, Z.; Vaisocherova, H.; Cheng, G.; Yang, W.; Xue, H.; Jiang, S.Y. Nonfouling Behavior of Polycarboxybetaine-Grafted Surfaces: Structural and Environmental Effects. Biomacromolecules 2008, 9, 2686-2692.

71. Wielema, T. A.; Engberts, J. B. F. N. Zwitterionic Polymers .2. Synthesis of a Novel Series of Poly(Vinylbetaines) and the Effect of the Polymeric Structure on the Solubility Behavior in Water. Eur. Polym.J. 1990, 26, 415-421.

72. Chevalier, Y.; Storet, Y.; Pourchet, S.; Leperchec, P. Tensioactive Properties of Zwitterionic Carboxybetaine Amphiphiles. Langmuir 1991, 7, 848-853.

73. Cao, B.; Li, L. L.; Tang, Q.; Cheng, G. The Impact of Structure on Elasticity, Switchability, Stability and Functionality of an All-in-One Carboxybetaine Elastomer. Biomaterials 2013, 34, 7592-7600.

74. Chen, H.; Zhao, C.; Zhang, M. Z.; Chen, Q.; Ma, J.; Zheng, J. Molecular Understanding and Structural-Based Design of Polyacrylamides and Polyacrylates as Antifouling Materials. Langmuir 2016, 32, 3315-3330.

75. Shao, Q.; Jiang, S.Y. Effect of Carbon Spacer Length on Zwitterionic Carboxybetaines. J.Phys. Chem. B 2013, 117, $1357-1366$.

76. Shao, Q.; Mi, L.; Han, X.; Bai, T.; Liu, S.; Li, Y.; Jiang, S. Differences in Cationic and Anionic Charge Densities Dictate Zwitterionic Associations and Stimuli Responses. J. Phys. Chem. B 2014, 118, 6956-6962.

77. Yu, X.; Liu, Z.; Janzen, J.; Chafeeva, I.; Horte, S.; Chen, W.; Kainthan, R. K.; Kizhakkedathu, J. N.; Brooks, D. E. Polyvalent 
Choline Phosphate as a Universal Biomembrane Adhesive. Nat. Mater. 2012, 11, 468-476.

78. Chen, X.; Yang, M.; Liu, B.; Li, Z.; Tan, H.; Li, J. Multilayer Choline Phosphate Molecule Modified Surface with Enhanced Cell Adhesion but Resistance to Protein Adsorption. Langmuir 2017, 33, 8295-8301.

79. Chen, X.; Chen, T.; Lin, Z.; Li, X. e.; Wu, W.; Li, J. Choline Phosphate Functionalized Surface: Protein-Resistant but CellAdhesive Zwitterionic Surface Potential for Tissue Engineering. Chem. Commun. 2015, 51, 487-490.

80. Li, S. Y.; Wang, F.; Li, X. Q.; Chen, J.; Zhang, X. H.; Wang, Y. C.; Liu, J. W. Dipole Orientation Matters: Longer-Circulating Choline Phosphate Than Phosphocholine Liposomes for Enhanced Tumor Targeting. ACS Appl. Mater. Interfaces 2017, 9 , 17736-17744.

81. Shao, Q.; He, Y.; White, A. D.; Jiang, S.Y. Difference in Hydration between Carboxybetaine and Sulfobetaine. J. Phys. Chem. B 2010, 114, 16625-16631.

82. Chen, S. F.; Li, L. Y.; Zhao, C.; Zheng, J. Surface Hydration: Principles and Applications toward Low-Fouling/Nonfouling Biomaterials. Polymer 2010, 51, 5283-5293.

83. Leng, C.; Hung, H. C.; Sun, S. W.; Wang, D. Y.; Li, Y. T.; Jiang, S. Y.; Chen, Z. Probing the Surface Hydration of Nonfouling Zwitterionic and PEG Materials in Contact with Proteins. ACS Appl. Mater. Interfaces 2015, 7, 16881-16888.

84. Pereira, A. D.; Rodriguez-Emmenegger, C.; Surman, F.; Riedel, T.; Alles, A. B.; Brynda, E. Use of Pooled Blood Plasmas in the Assessment of Fouling Resistance. RSC Adv. 2014, 4, 2318-2321.

85. Xiao, S.; Zhang, Y.; Shen, M.; Chen, F.; Fan, P.; Zhong, M.; Ren, B.; Yang, J.; Zheng, J. Structural Dependence of SaltResponsive Polyzwitterionic Brushes with an Anti-Polyelectrolyte Effect. Langmuir 2018, 34, 97-105.

86. Yang, J.T.; Chen, H.; Xiao, S. W.; Shen, M. X.; Chen, F.; Fan, P.; Zhong, M. Q.; Zheng, J. Salt-Responsive Zwitterionic Polymer Brushes with Tunable Friction and Antifouling Properties. Langmuir 2015, 31, 9125-9133.

87. Zhang, J.; Kou, R.; Liu, G. M. Effect of Salt Concentration on the pH Responses of Strong and Weak Polyelectrolyte Brushes. Langmuir 2017, 33, 6838-6845.

88. Zhang,Z.; Moxey, M.;Alswieleh,A.; Morse,A.J.; Lewis,A.L.; Geoghegan, M.; Leggett, G.J. Effect of Salt on PhosphorylcholineBased Zwitterionic Polymer Brushes. Langmuir 2016, 32, 5048-5057.

89. Zhao, B.;Yuan, G.; Chu, X.; Yang, J.; Zhao, J. Response of a Permanently Charged Polyelectrolyte Brush to External Ions: The Aspects of Structure and Dynamics. Langmuir 2018, 34, 6757-6765.

90. Wang, Z.; van Andel, E.; Pujari, S. P.; Feng, H.; Dijksman, J. A.; Smulders, M. M. J.; Zuilhof, H. Water-Repairable Zwitterionic Polymer Coatings for Anti-Biofouling Surfaces. J. Mater. Chem. B 2017, 5, 6728-6733.

91. Shao, Q.; Jiang, S.Y. Influence of Charged Groups on the Properties of Zwitterionic Moieties: A Molecular Simulation Study. J.Phys. Chem. B 2014, 118, 7630-7637.

92. Xiang, Y.; Xu, R.-G.; Leng, Y. Molecular Simulations of the Hydration Behavior of a Zwitterion Brush Array and Its Antifouling Property in an Aqueous Environment. Langmuir 2018, 34, 2245-2257.

93. Mehmood, S.; Allison, T. M.; Robinson, C. V. Mass Spectrometry of Protein Complexes: From Origins to Applications. Annu. Rev. Phys. Chem. 2015, 66, 453-474.

94. Trinkle-Mulcahy, L.; Boulon, S.; Lam, Y.W.; Urcia, R.; Boisvert, F. M.; Vandermoere, F.; Morrice, N. A.; Swift, S.; Rothbauer, U.; Leonhardt, H.; Lamond, A. Identifying Specific Protein Interaction Partners Using Quantitative Mass Spectrometry and Bead Proteomes. J.Cell Biol. 2008, 183, 223-239.

95. Trilling, A. K.; Hesselink, T.; van Houwelingen, A.; Cordewener, J. H. G.; Jongsma, M. A.; Schoffelen, S.; van Hest, J. C. M.; Zuilhof, H.; Beekwilder, J. Orientation of Llama Antibodies Strongly Increases Sensitivity of Biosensors. Biosens. Bioelectron. 2014, 60, 130-136.

96. Duval, F.; van Beek, T. A.; Zuilhof, H. Key Steps Towards the Oriented Immobilization of Antibodies Using Boronic Acids. The Analyst 2015, 140, 6467-6472.

97. Shen, M.; Rusling, J. F; Dixit, C. K. Site-Selective Orientated Immobilization of Antibodies and Conjugates for Immunodiagnostics Development. Methods 2017, 116, 95-111. 
98. Dunham, W. H.; Mullin, M.; Gingras, A. C. Affinity-Purification Coupled to Mass Spectrometry: Basic Principles and Strategies. Proteomics 2012, 12, 1576-1590.

99. Pina, A. S.; Lowe, C. R.; Roque, A. C. A. Challenges and Opportunities in the Purification of Recombinant Tagged Proteins. Biotechnol.Adv. 2014, 32, 366-381.

100.Gould, H. J.; Sutton, B. J. IgE in Allergy and Asthma Today. Nat. Rev. Immunol. 2008, 8, 205-217.

101.Aggarwal, P.; Hall, J. B.; McLeland, C. B.; Dobrovolskaia, M. A.; McNeil, S. E. Nanoparticle Interaction with Plasma Proteins as It Relates to Particle Biodistribution, Biocompatibility and Therapeutic Efficacy. Adv. Drug Delivery Rev. 2009, 61, 428-437.

102.Blaszykowski, C.; Sheikh, S.; Thompson, M. A Survey of State-of-the-Art Surface Chemistries to Minimize Fouling from Human and Animal Biofluids. Biomater. Sci. 2015, 3, 1335-1370.

103.Rodriguez-Emmenegger, C.; Houska, M.; Alles, A. B.; Brynda, E. Surfaces Resistant to Fouling from Biological Fluids: Towards Bioactive Surfaces for Real Applications. Macromol. Biosci. 2012, 12, 1413-1422.

104. Vasti, C.; Bonnet, L.V.; Galiano, M. R.; Rojas, R.; Giacomelli, C. E. Relevance of Protein-Protein Interactions on the Biological Identity of Nanoparticles. Colloids Surf., B 2018, 166, 330-338.

105. Mosser, D. M.; Edwards, J. P. Exploring the Full Spectrum of Macrophage Activation. Nat. Rev. Immunol. 2008, 8, 958-969.

106. Guyre, P. M.; Graziano, R. F.; Goldstein, J.;Wallace, P. K.; Morganelli, P. M.; Wardwell, K.; Howell, A. L. Increased Potency of FC-Receptor-Targeted Antigens. Cancer Immunol. Immun. 1997, 45, 146-148.

107.Zhang, Y.; Liu, S.; Yu, Y.; Zhang, T.; Liu, J.; Shen, Q.; Cao, X. Immune Complex Enhances Tolerogenecity of Immature Dendritic Cells Via FcgammaRIIb and Promotes FcgammaRIIb-Overexpressing Dendritic Cells to Attenuate Lupus. Eur. J. Immunol. 2011, 41, 1154-1164.

108. Champion, J. A.; Walker, A.; Mitragotri, S. Role of Particle Size in Phagocytosis of Polymeric Microspheres. Pharm. Res. 2008, $25,1815-1821$.

109.Xiang, S. D.; Scholzen, A.; Minigo, G.; David, C.; Apostolopoulos, V.; Mottram, P. L.; Plebanski, M. Pathogen Recognition and Development of Particulate Vaccines: Does Size Matter? Methods 2006, 40, 1-9.

110.Garcia, K. P.; Zarschler, K.; Barbaro, L.; Barreto, J. A.; O’Malley, W.; Spiccia, L.; Stephan, H.; Graham, B. Zwitterionic-Coated "Stealth" Nanoparticles for Biomedical Applications: Recent Advances in Countering Biomolecular Corona Formation and Uptake by the Mononuclear Phagocyte System. Small 2014, 10, 2516-2529.

111.Trapiella-Alfonso, L.; Pons, T.; Lequeux, N.; Leleu, L.; Grimaldi, J.; Tasso, M.; Oujagir, E.; Seguin, J.; d’Orlyé, F.; Girard, C.; Doan, B.-T.; Varenne, A. Clickable-Zwitterionic Copolymer Capped-Quantum Dots for in Vivo Fluorescence Tumor Imaging. ACS Appl. Mater. Interfaces 2018, 10, 17107-17116

112.Xiong, Z. J.; Wang, Y.; Zhu, J. Y.; Li, X.; He, Y.; Qu, J.; Shen, M. W.; Xia, J. D.; Shi, X. Y. Dendrimers Meet Zwitterions: Development of a Unique Antifouling Nanoplatform for Enhanced Blood Pool, Lymph Node and Tumor CT Imaging. Nanoscale 2017, 9, 12295-12301.

113.Lu, A. J.;Wu, Z. Z.; Luo, X. L.; Li, S. M. Protein Adsorption and Macrophage Uptake of Zwitterionic Sulfobetaine Containing Micelles. Colloids Surf., B 2018, 167, 252-259.

114.Wen, N.; Lü, S.; Gao, C.; Xu, X.; Bai, X.;Wu, C.; Ning, P.; Liu, M. Glucose-Responsive Zwitterionic Dialdehyde Starch-Based Micelles with Potential Anti-Phagocytic Behavior for Insulin Delivery. Chem. Eng.J. 2018, 335, 52-62.

115.Zhang, K.-L.; Zhou, J.; Zhou, H.;Wu, Y.; Liu, R.;Wang, L.-L.; Lin, W.-W.; Huang, G.; Yang, H.-H. Bioinspired “Active” Stealth Magneto-Nanomicelles for Theranostics Combining Efficient MRI and Enhanced Drug Delivery. ACS Appl. Mater. Interfaces 2017, 9, 30502-30509.

116.Dong, Z.; Mao, J.; Yang, M.; Wang, D.; Bo, S.; Ji, X. Phase Behavior of Poly(Sulfobetaine Methacrylate)-Grafted Silica Nanoparticles and Their Stability in Protein Solutions. Langmuir 2011, 27, 15282-15291.

117.Zheng, L. C.; Sundaram, H. S.; Wei, Z.Y.; Li, C. C.; Yuan, Z. F. Applications of Zwitterionic Polymers. React. Funct. Polym. 2017, $118,51-61$.

118.Liang, B.; Zhang, G.; Zhong, Z.; Huang, Y.; Su, Z. Superhydrophilic Anti-Icing Coatings Based on Polyzwitterion Brushes. 
Langmuir 2018, DOI: 10.1021/acs.langmuir.8b01009

119. Mi, L.; Jiang, S. Integrated Antimicrobial and Nonfouling Zwitterionic Polymers. Angew. Chem. Int. Ed. Engl. 2014, 53, 17461754.

120.Li, B.;Yu, B.; Ye, Q.; Zhou, F. Tapping the Potential of Polymer Brushes through Synthesis. Acc. Chem. Res. 2014, 48, $229-237$. 


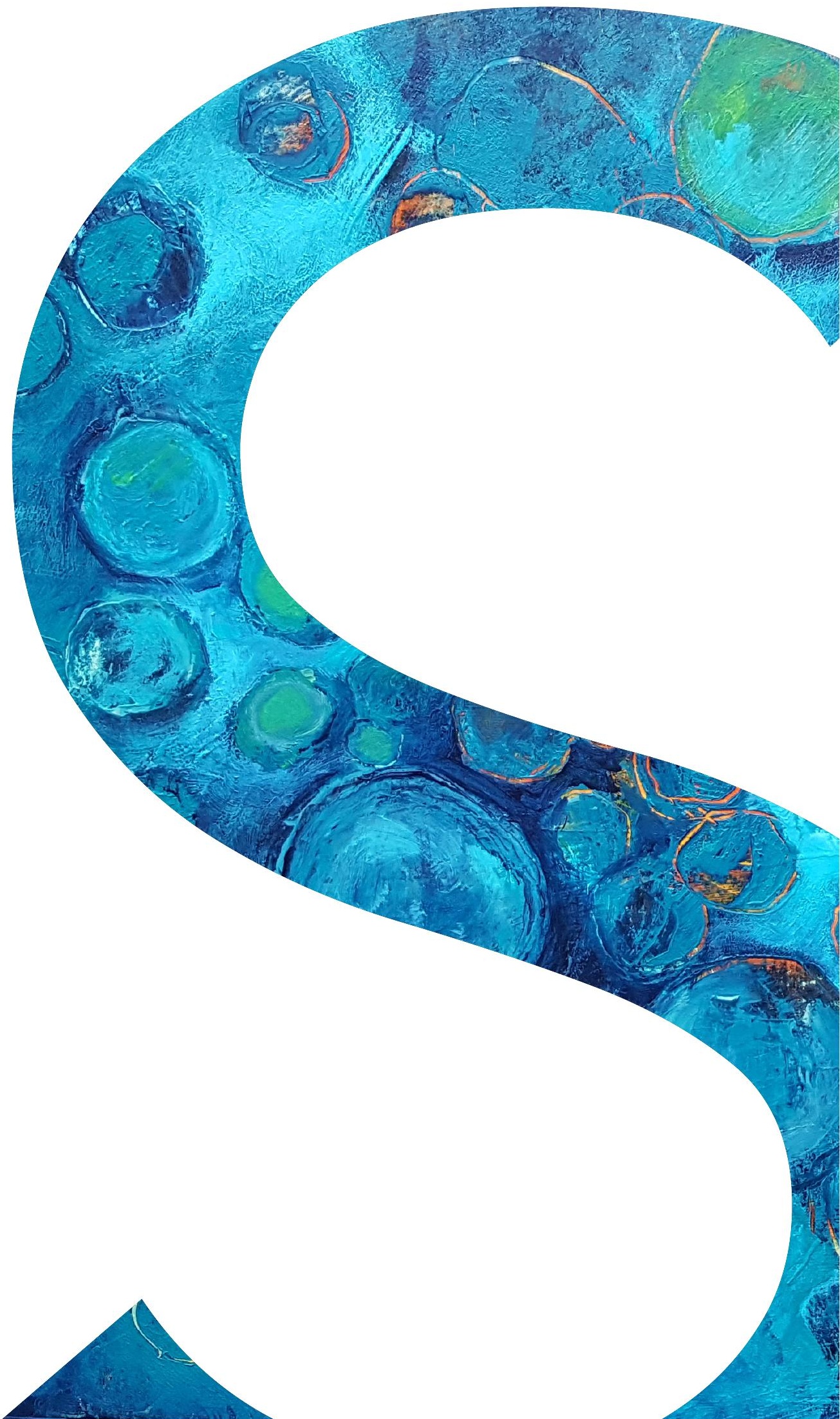




\section{Summaries}

Summary (English)

Samenvatting (Nederlands) 
$236 \mid$ 


\section{Summary}

The non-specific adsorption of biomolecules, often referred to as fouling, is a recurring issue in many biomedical and bioanalytical applications. For example, the non-specific adsorption of proteins diminishes the signal-to-noise ratio of biosensing systems, while protein coronas formed on drug delivery vehicles hamper the drug to reach its target site due to fast removal by phagocytic cells. Zwitterionic polymer brushes have been brought forward as excellent candidates to prevent fouling. This thesis provides tools and insights to improve antifouling performance in general, to efficiently introduce functionality to zwitterionic polymer brushes and to transfer existing knowledge of antifouling coatings on flat surfaces onto microbeads. In addition, two applications for the antifouling zwitterionic polymer-coated microbeads are also presented.

Chapter 1 provides an introduction to antifouling coatings in general and to zwitterionic polymer brushes in particular. This chapter also introduces the concept of a romantic surface: a surface that repels all biomolecules except that special one it is interested in. Chapter 2 describes a novel approach on how to create such romantic surfaces. An azide-functionalized sulfobetaine is introduced that enables the 3D-functionalization of fully zwitterionic polymer brushes. The number of built-in functional groups could be tuned by the co-polymerization of this novel azido-SB monomer with a standard sulfobetaine. The incorporated azide moieties can then be used to couple recognition units of choice using "click" chemistry. Functionalization of the brushes with biotin units enabled the specific binding of avidin without the interference of the non-specific binding of fibrinogen.

Particles of various sizes are being used for a wide range of biomedical application, including drug delivery, imaging, cells sorting and as biosensing platform. As on flat surfaces, fouling compromises the performance of these particles. Chapter 3 describes a methodology to efficiently coat microbeads with zwitterionic antifouling coatings. Top functionalization of the polymer brush chain ends was used to introduce various functional groups, including biotin and mannose moieties. The beads were able to specifically bind streptavidin and ConA, respectively, in the presence of a $10 \%$ serum solution. Flow cytometry was shown to be a powerful tool to evaluate both non-specific as well as specific binding of proteins in a quick and automated manner. The methodology developed in chapter 3 was used for chapters 4, 5 and 6 .

Chapter $\mathbf{4}$ presents a systematic comparison of various antifouling polymer brushes to deepen the understanding on the exact relationship between monomer structure and antifouling performance. Sulfobetaines with three methylene groups between opposite charges (SB-3) have often been compared to carboxybetaines with two separating methylene groups (CB-2). In chapter 4, for the first time, a direct comparison was made between SB-3, CB-2 and SB-2 (a sulfobetaine with two separating methylene groups), revealing that both carbon spacer length as well as the nature of the charged groups have an influence on the antifouling capacity. A phosphoscholine and a hydroxyl acrylamide monomer were also shown to produce excellent 
antifouling surfaces. It was also demonstrated that the bead-based platform, as described in chapter 3, provides a great tool to evaluate antifouling performance.

Immunoprecipitation followed by mass spectrometry (IP-MS) is a powerful technique to study protein-protein interactions. The differentiation between true protein interactors and proteins that bind non-specifically is, however, often challenging. Chapter $\mathbf{5}$ describes the use of zwitterionic polymer brush-coated microbeads to reduce background signals in IPMS experiments. The azide-functionalized sulfobetaine monomer that was introduced in chapter 2, was used to enable the immobilization of antibodies via click chemistry. Antifouling anti-GFP beads were produced, which showed similar levels of GFP capture as commercially available anti-GFP beads, but with strongly reduced non-specific protein binding. In an IP-MS experiment many contaminating proteins were identified with the commercially available antiGFP beads, whereas the antifouling anti-GFP beads were able to almost exclusively purify the GFP-tagged bait protein and its known interaction partners.

The non-specific adsorption of proteins onto intravenous injected particles often leads to the rapid clearance from the blood stream by phagocytic cells like macrophages and other antigen-presenting cells. This drastically hampers the in vivo performance of the particles. Chapter 6 describes the use of antifouling zwitterionic polymer brush-coated microbeads to prevent phagocytosis by murine macrophages. The functionalization of the antifouling beads with proteins did not result in a significant increase in phagocytic uptake. On the other hand, when the beads were specifically targeted to the macrophages by the immobilization of $\operatorname{IgG}_{2 \mathrm{a}}$ antibodies, the beads were actively internalized via Fc receptor-mediated phagocytosis. This chapter demonstrates the high potential of functionalized zwitterionic polymer brush-coated particles for in vitro and in vivo applications.

Chapter 7 provides a general discussion on the most important achievements that are outlined within this thesis. Special attention is given to suggestions for future research. Novel monomers are proposed that may lead to improved antifouling capacity. In addition, potential applications of antifouling beads are discussed. 


\section{Samenvatting}

De aspecifieke adsorptie van eiwitten en ander biomateriaal aan oppervlakken, gedefinieerd als (bio)fouling, vormt een terugkerend probleem in veel biomedische toepassingen. Het leidt bijvoorbeeld tot een verslechterde signaal-ruis verhouding in diagnostische testen en kan verhinderen dat een bepaald medicijn op de juiste plek in het lichaam terecht komt. Eiwitafstotende coatings, en dan met name coatings die bestaan uit zogenaamde zwitterionische polymeerlagen, kunnen hier een oplossing bieden. Vanwege de hoge dichtheid aan positieve en negatieve ladingen kan dit type coatings zeer goed water vasthouden. De waterlaag voorkomt, door de fysieke en energetische barrière, dat eiwiten aan de coatings kunnen binden. In dit proefschrift wordt uiteengezet hoe deze polymeerlagen verder kunnen worden verbeterd en hoe herkenningselementen aan de coatings vastgezet kunnen worden die in staat zijn specifiek een molecuul te binden. Op deze manier wordt een 'romantisch' oppervlak gecreëerd: een oppervlak dat al het biomateriaal afstoot, behalve dat ene molecuul waar het naar op zoek is. De opgedane kennis voor het maken van romantische, eiwitafstotende coatings op vlakke oppervlakken is vervolgens gebruikt voor het coaten van bolvormige microbeads. De antifouling microbeads kunnen voor verschillende toepassingen binnen de biochemie/ biomedische wereld gebruikt worden.

Hoofdstuk 1 geeft een algemene introductie over eiwitafstotende coatings en introduceert het concept romantisch oppervlak. Hoofdstuk 2 beschrijft een nieuwe aanpak hoe zulke romantische oppervlakken gecreëerd kunnen worden. Hiertoe wordt een zwitterionisch monomeer (sulfobetaine) geïntroduceerd die is uitgerust met een reactieve azide groep. De azide groepen maken het mogelijk om over de hele hoogte van de polymeerlaag een gewenst herkenningselement vast te zetten mét behoud van het zwitterionisch karakter van de polymeerlaag. De mate van inbouwing van deze herkeningselementen kan worden gecontroleerd door het nieuwe azido-sulfobetaine monomeer te copolymeriseren met een standaard zwitterionisch monomeer. De ingebouwde azide groepen kunnen vervolgens worden gebruikt voor het koppelen van herkenningselementen met behulp van klikchemie. Biotine dat op deze manier aan de polymeerlaag gekoppeld was, bleek in staat om zeer selectief het eiwit avidine te binden maar tegelijkertijd het ook aanwezige ('plakkerige') eiwit fibrinogeen af te stoten.

In veel biomedische toepassingen wordt gebruikt gemaakt van zogenaamde beads (bolvormige polymeerdeeltjes variërend van enkele nanometers tot enkele honderden micrometers in grootte), voor onder andere drug delivery, imaging, en voor de diagnostiek. Fouling zorgt net als op vlakke oppervlakken voor een verminderde specificiteit en daarmee toepasbaarheid van deze beads. Hoofdstuk 3 beschrijft hoe magnetische beads van enkele micrometers groot kunnen worden voorzien van een zwitterionische polymeerlaag. Aan de uiteinden van de polymeerketens konden verschillende soorten functionele groepen worden vastgezet, waaronder biotine en mannose verbindingen. Met deze microbeads konden specifiek respectievelijk streptavidine en conanavaline A uit een 10\% serum oplossing gebonden worden. Flowcytometrie werd hierbij geïntroduceerd als een nieuwe techniek voor het op een snelle en geautomatiseerde wijze 
kwantitatief meten van zowel specifieke als aspecifieke (fouling) eiwitadsorptie. De methodiek beschreven in hoofdstuk 3 werd ook gebruikt voor de hoofdstukken 4, 5 en 6.

In hoofdstuk 4 worden verschillende polymeerlagen met elkaar vergeleken om de relatie tussen monomeerstructuur en eiwitafstotend vermogen te kunnen bepalen. Sulfobetaines met drie methyleengroepen tussen de tegengestelde ladingen (SB-3) worden vaak vergeleken met carboxybetaines met twee methyleengroepen (CB-2). In hoofdstuk 4 wordt voor de eerste keer een directe vergelijking gemaakt tussen SB-3, CB-2 en SB-2 (een sulfobetaine met 2 methyleengroepen tussen de tegengestelde ladingen). Hieruit bleek dat zowel de ketenlengte tussen de tegengestelde ladingen als het type anion bepalend zijn voor de mate waarin eiwitadsorptie plaatsvindt. Daarnaast hebben we ook gekeken naar een zwitterionisch phosphoryl chloride en een niet zwitterionisch hydroxyl metacrylamide monomeer, beide bleken over zeer goede antifouling eigenschappen te beschikken.

Immunoprecipitatie gevolgd door massa spectrometrie (IP-MS) is een veelgebruikte op beads gebaseerde techniek voor het bestuderen van eiwit-eiwit interacties. Het blijft echter zeer uitdagend om onderscheid te maken tussen echte interacties en eiwitten die aspecifiek blijven plakken aan de beads. In Hoofdstuk 5 wordt beschreven hoe de aanwezigheid van een een eiwitafstotende polymeerlaag op een microbead de hoeveelheid achtergrondsignaal als gevolg van aspecifieke eiwitbinding in IP-MS experimenten kan reduceren. Het azide bevattende monomeer, zoals beschreven in hoofdstuk 2, werd hierbij gebruikt voor het vastzetten van antilichamen op de zwitterionische coating. Microbeads voorzien van GFP antilichamen lieten net zoveel GFP binding zien als commercieel verkrijgbare beads, maar met een sterk gereduceerde hoeveelheid aspecifieke eiwitbinding. Dit zorgde voor een sterk verbeterde identificatie van een GFP fusie eiwit in een IP-MS experiment.

De aspecifieke binding van eiwitten aan medicijndragers leidt vaak tot een snelle verwijdering van deze deeltjes uit de bloedbaan door fagocyterende cellen van het immuunsysteem, nog voor dat de deeltjes de juiste plek kunnen bereiken. Hoofdstuk 6 laat zien dat door het introduceren van de zwitterionische coating op microbeads kan worden voorkomen dat deze microbeads worden gefagocyteerd door macrofagen. Wanneer de microbeads werden voorzien van specifieke $\operatorname{IgG}_{2 \mathrm{a}}$ antilichamen konden de macrofagen deze microbeads herkennen en daardoor specifiek opnemen met behulp van Fc-receptor gemedieerde fagocytose. De microbeads kunnen dus naar gelang met rust worden gelaten of specifiek worden opgenomen. Dit illustreert de potentie van dit soort polymeer gecoate microbeads voor in vitro en in vivo toepassingen.

Hoofdstuk 7 omvat een algemene discussie over de belangrijkste bevindingen die beschreven zijn in de voorafgaande hoofdstukken van dit proefschrift, waarbij met name aandacht is voor suggesties voor verder onderzoek. 


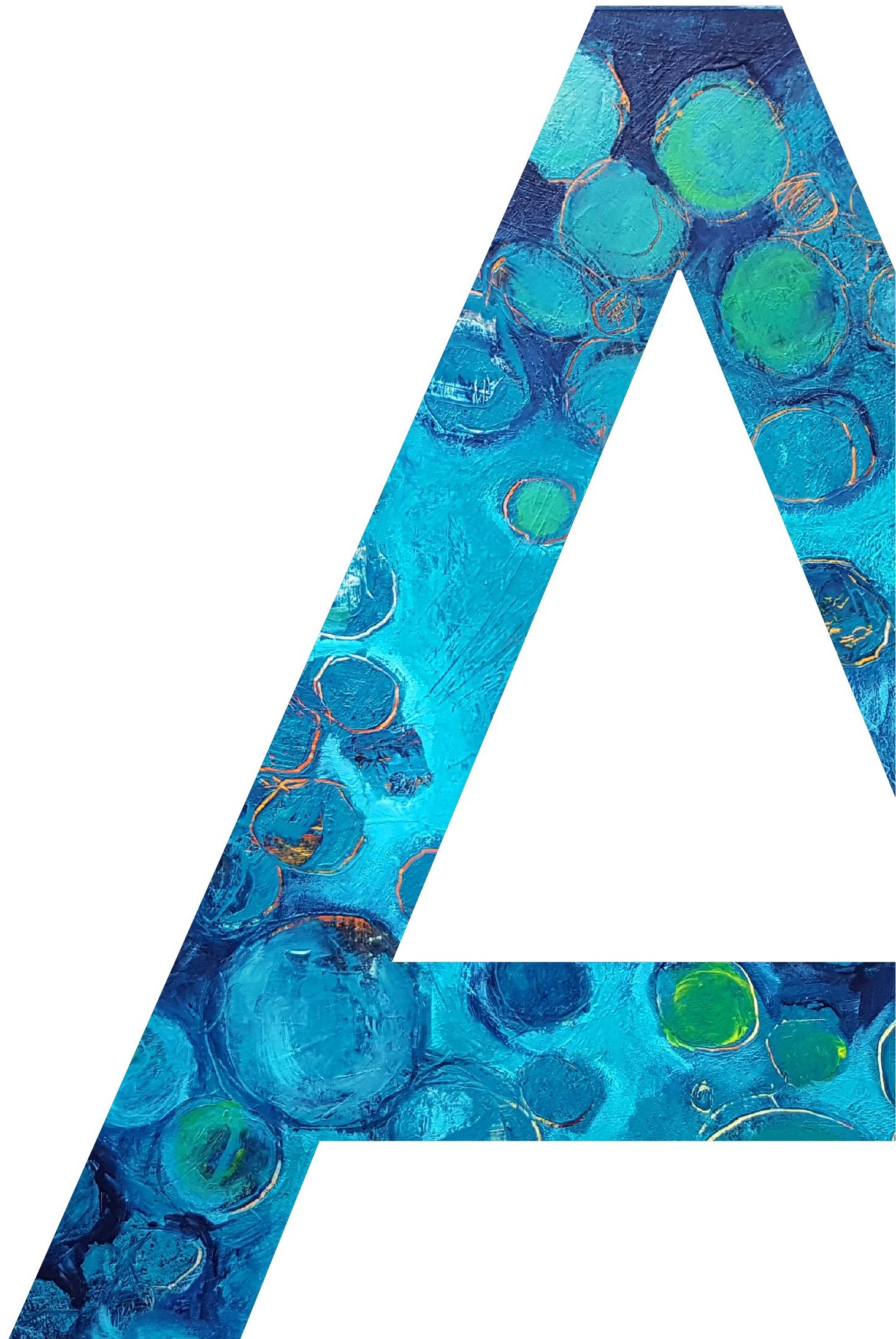




\section{About the Author}

Curriculum Vitae

List of publications

Overview of completed training activities

Acknowledgements 
244 | 


\section{Curriculum vitae}

Esther van Andel was born on April 18, 1990 in Zwolle, The Netherlands. She finished high school (Meander College, Zwolle) in 2008 after which she moved to Wageningen to study Molecular Life Sciences. She conducted her BSc thesis research at the Laboratory of Organic Chemistry where she got introduced to surface chemistry (supervisors: S. P. Pujari and H. Zuilhof). The focus during her BSc degree was on chemistry, while she shifted towards more biological subjects during her MSc degree (specialization: Biomedical Research). As part of her MSc program, she performed a short internship in education, did her MSc thesis research at the Laboratory of Virology (supervisors: V. I. D. Ros and M. M. van Oers) and an internship at the University of Copenhagen on cancer immunology (supervisors: F. Uhlenbrock

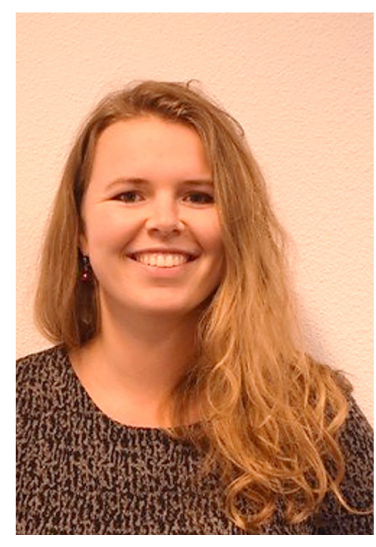
and S. Skov). Parallel to her study she worked as a student assistant to supervise practicals for the course Cell Biology and Health. Esther obtained her BSc degree (cum laude) in 2011 and her MSc degree (cum laude) in 2013.

In 2014 she started her PhD at the Laboratory of Organic Chemistry and the Cell Biology and Immunology Group in Wageningen under the supervision of M. M. J. Smulders, E. J. Tijhaar, H. F. J. Savelkoul and H. Zuilhof. The most important results of this work are described in this thesis.

Parallel to finishing up her PhD thesis, Esther started as a post-doctoral fellow at the Laboratory of Organic Chemistry under the supervision of H. Zuilhof, and in collaboration with Roche Diagnostics and ETH Zürich. As of January 2019 she will start as a clinical chemist in training at the Leiden University Medical Center (LUMC). 


\section{List of publications}

van Andel, E.; Cuenca-Escalona, J.; van der Zanden, S.; Smulders, M. M. J.; Zuilhof, H.; Savelkoul, H. F. J.;Tijhaar, E. J.Targeted Phagocytosis of Functionalized Stealth Microbeads by Macrophages. Manuscript in preparation.

van Andel, E.; Roosjen, M.; van der Zanden, S.; Lange, S. C.; Weijers, D.; Smulders, M. M. J.; Savelkoul, H. F. J.; Zuilhof, H.;Tijhaar, E. J. Specific Protein Identification by ImmunoprecipitationMass Spectrometry Using Antifouling Microbeads. 2018, Manuscript in preparation.

van Andel, E.; Lange, S. C.; Pujari, S. P.;Tijhaar, E. J.; Smulders, M. M. J.; Savelkoul, H. F. J.; Zuilhof, H. Systematic Comparison of Zwitterionic and Non-Zwitterionic Antifouling Polymer Brushes on a Bead-Based Platform. Langmuir 2018, in press, DOI: 10.1021/acs.langmuir.8b01832.

van Andel, E.; de Bus, I.; Tijhaar, E. J.; Smulders, M. M. J.; Savelkoul, H. F. J.; Zuilhof, H. Highly Specific Binding on Antifouling Zwitterionic Polymer-Coated Microbeads as Measured by Flow Cytometry. ACS Appl. Mater. Interfaces 2017, 9, 38211-38221.

Wang, Z.; van Andel, E.; Pujari, S. P.; Feng, H.; Dijksman, J. A.; Smulders, M. M. J.; Zuilhof, H. Water-Repairable Zwitterionic Polymer Coatings for Anti-Biofouling Surfaces. J. Mater. Chem. B 2017, 5, 6728-6733. This article features on the back cover of J. Mater. Chem. B, volume 5, issue 33, 2017.

Chatzipetrou, M.; Massaouti, M.; Tsekenis, G.; Trilling, A. K.; van Andel, E.; Scheres, L.; Smulders, M. M. J.; Zuilhof, H.; Zergioti, I. Direct Creation of Biopatterns via a Combination of LaserBased Techniques and Click Chemistry. Langmuir 2017, 33, 848-853.

Lange, S. C.*; van Andel, E.*; Smulders, M. M. J.; Zuilhof, H. Efficient and Tunable ThreeDimensional Functionalization of Fully Zwitterionic Antifouling Surface Coatings. Langmuir 2016, 32, 10199-10205. *Contributed equally. This paper was selected as ACS Editors' Choice, and also features on the front cover of Langmuir, volume 32, issue 40, 2016.

Uhlenbrock, F.; van Andel, E.; Andresen, L.; Skov, S. A Conserved WW Domain-Like Motif Regulates Invariant Chain-Dependent Cell-Surface Transport of the NKG2D Ligand ULBP2. Mol. Immunol. 2015, 66, 418-427.

Pujari, S. P.; van Andel, E.; Yaffe, O.; Cahen, D.; Weidner, T.; van Rijn, C. J. M.; Zuilhof, H. MonoFluorinated Alkyne-Derived SAMs on Oxide-Free Si(111) Surfaces: Preparation, Characterization and Tuning of the Si Workfunction. Langmuir 2013, 29, 570-580. 


\section{Overview of completed training activities}

\begin{tabular}{|c|c|c|}
\hline Discipline-specific activities & Organizing institute & Year \\
\hline Advanced Immunology: Infection and Immunity & UU Graduate School & 2015 \\
\hline FACS Bootcamp & Excyte eBioscience & 2015 \\
\hline Advanced Chemistry \& Advanced Organic Chemistry & ORC & 2014-2018 \\
\hline CHAINS (CHemistry As INnovating Science) & NWO & 2014-2016 \\
\hline Wageningen Symposium on Organic Chemistry & $\mathrm{KNCV}$ & 2014,2016 \\
\hline NanoCity & NanoNextNL & $2015-2016$ \\
\hline NanoMedicine days & NanoNextNL & 2015 \\
\hline NVVI Lunteren (Immunology symposium) & NVVI & 2017 \\
\hline Biosensing Technology Conference (Zürich) & WASET & 2017 \\
\hline ACS National Meeting and Exposition (New Orleans) & ACS & 2018 \\
\hline General courses & Organizing institute & Year \\
\hline VLAG PhD week & VLAG & 2014 \\
\hline Project \& Time Management & WGS & 2014 \\
\hline IP and Valorisation Awareness & NanoNextNL & 2015 \\
\hline Speed Reading Course & VLAG & 2015 \\
\hline Scientific Artwork with Photoshop and Illustrator & WUR Library & 2016 \\
\hline Career Perspectives & WGS & 2017 \\
\hline Optionals & Organizing institute & Year \\
\hline Group meetings and seminars & $\mathrm{ORC} / \mathrm{CBI}$ & 2014-2018 \\
\hline Preparation PhD research proposal & ORC & 2014 \\
\hline $\begin{array}{l}\text { Convener at PhD Symposium: Connecting Ideas - } \\
\text { Combining Forces }\end{array}$ & WPC & 2015 \\
\hline PhD study trips (Canada and Denmark/Sweden) & ORC & 2015,2017 \\
\hline Organization PhD study trip (Denmark/Sweden) & ORC & 2017 \\
\hline
\end{tabular}


248 | 


\section{Acknowledgements}

Dear all,

After 4.5 years of hard work there is finally this thesis. Cliché but true: I couldn't have done it on my own... I would therefore like to thank everyone that contributed to this work, whether it is via a significant scientific contribution or just by a kind gesture; I am grateful to all of you. THANKS EVERYONE!

To start with, I would like to thank my promotors Han Zuilhof and Huub Savelkoul. Of course I am thankful for your guidance during the last past years, but foremost for being so openminded to start, based on my wishes and doubts, a new collaboration between ORC and CBI. I felt comfortable between the two 'worlds' and I like to believe that we all learned a lot from this collaboration. Thanks a lot for this opportunity! Then my two co-promotors: Maarten Smulders and Edwin Tijhaar. What I really appreciated is that you were both very approachable and that the communication lines were short. Maarten, as I started as one of your first PhD students and only shortly after you started at ORC, we had to figure out many things together. I often asked you for advise (not per se on scientific content) and by that you became a true mentor for me; thanks for that! My apologies for walking in your office maybe a little bit too often. Edwin, without you the ORC-CBI collaboration would not have been as fruitful as it has been now, thanks for your practical input in the lab and the many ideas that became part of this thesis! Finally, I would like to thank my supervisors for giving me freedom and responsibility within this project, as well as for the support in pursuing my next steps in my career.

A good working atmosphere is very important to me and I am in this respect grateful for all the nice colleagues I had the chance to work with. I am happy that I could be part of both worlds (that at first caused some culture shocks when going back and forth between CBI/EZO and ORC, but in the end did not seem so different anymore). I hope to keep in touch with many of you! As my home basis was at ORC, a special thanks to the colleagues from CBI/EZO that made me feel welcome: much appreciated! I won't name all of you individually (sorry) as I can only do you short while doing so. Instead, I want to thank you collectively for (you should be able to mark the phrases that are directed to you):

- the many fun activities: lab trips, $\mathrm{PhD}$ weekends, $\mathrm{PhD}$ study trips, pub quizzes, (Christmas) diners, We-Days, 'Sinterklaas' evenings, board games, PhD parties, 'wadlopen', etc.

- the regular 'corridor meetings'

- being my partners in crime in getting GOOD coffee from Campus Plaza

- asking me to be your paranimph and wanting to be mine

- letting me abuse the \#colleagueswithoutborders WhatsApp group as a helpdesk and letting me in and out Zodiac labs when my WUR card was once again not working or forgotten

- letting me fall of your horse

- fighting for (and decorating!) my desk at CBI 
- the interest in my personal life and the new friendships that were born from this

- the silly jokes and daily portions of nonsense

- the advice on my future career and/or life in general

- carpooling

- being the best office mates I could have wished for

- ordering chemicals/reagents

- planning 'impossible to plan' meetings with all my supervisors

- answering all my questions in and outside of the lab

- forming a good teaching team

- guiding me through the PhD thesis hand-in phase by telling me when to do what/where

- inspiring me to use phrases like this

- performing synthesis for me

- never being able to eat cheese fondue anymore without certain memories

- $\quad \ldots$

The data presented in this thesis could not have been obtained without the assistance from technical staff members. Special thanks to Ben Meijer for helping me and my students with flow cytometry and anything related to that, Jan Willem Borst for his expertise in confocal microscopy, Sidharam Pujari and Barend van Lagen for teaching me all the surface analysis tools, Remco Fokkink for his help with the equipment from PCC (e.g. reflectometry and zeta potential measurements), and René Achterberg for helping me with the SPR experiments, and Jelmer van der Rijst for fixing basically everything and building the jealous-making set up in my fume hood. I would also like to thank all other technical staff for their support and efforts to let the labs run smoothly.

Then I would also like to thank my students: Ian de Bus, Marlene Fürher, Imke Reith and Myrna Bunte, Stef van der Zanden and Jorge Cuenca Escalona. Many thanks for your enthusiasm, hard work and great results; I was very lucky with you as students! I am therefore also pleased that much of your work ended up in my thesis and papers.

In addition, I want to say thanks to my collaborators at Biochemistry (BIC): Mark Roosjen, Dolf Weijers and Sjef Boeren. We had a noticeable knowledge gap but I think we made a very nice paper together. Thanks for your patience and efforts! Also a big thanks to my collaborators/ Co-authors at ORC: Stefanie Lange and Sidharam Pujari. Steff, I really enjoyed working with you as well as all the time we spent together outside of work. I learned a lot from you and the projects would not have worked out without your efforts. Besides that, I think we were a great team :-). Many thanks! Sidhu, ORC without you is no ORC and I guess that says enough. Thanks for all your help!

Besides my colleagues, I would also like to thank my house mates (both at H90 and Tigris), friends and family. I would like to thank you for being there for me, providing me with lovely meals (and eating mine :-)), and supporting me in obtaining this $\mathrm{PhD}$ thesis. Above all, I want to say thanks to Jan. Many do not understand our relationship, but I truly wish anyone such a caring partner as you. I admire your endless support and positivity and I cannot think of a 
single time that you complained of me being home late, being tired from work or letting the kitchen explode.

At last I would like to thank my mom for, among many other things, making the painting for the cover and Eva Verhoef for the help with the lay-out of this booklet. A book is first judged by its cover!

Best wishes to all of you!

Esther 
The research and training activities described in this thesis were financially supported by NanoNextNL, program 3E "Integrated microsystems for biosensing".

Financial support from Wageningen University for printing this thesis is gratefully acknowledged.

Cover image by Marijke van der Blom

Lay out by Eva Verhoef and Esther van Andel 
